\title{
Literature review of baseline information on RNAi to support the environmental risk assessment of RNAi-based GM plants
}

\author{
Olivier Christiaens ${ }^{1}$, Teodora Dzhambazova², Kaloyan Kostov², Salvatore Arpaia ${ }^{3}$, \\ Mallikarjuna Reddy Joga ${ }^{1}$, Isabella Urru ${ }^{3}$, Jeremy Sweet ${ }^{4}$, Guy Smagghe ${ }^{1}$ \\ ${ }^{1}$ Ghent University, Belgium \\ ${ }^{2}$ Agrobioinstitute (ABI), Sofia, Bulgaria \\ ${ }^{3}$ Italian National Agency for New Technologies, Energy and Sustainable Economic Development \\ (ENEA), Italy \\ ${ }^{4} \mathrm{JT}$ Environmental Consultants Ltd, Cambridge, UK
}

\begin{abstract}
This report is the outcome of an EFSA procurement (OC/EFSA/GMO/2015/02) which aims at reviewing relevant scientific information on RNA interference (RNAi) that could serve as baseline information for the environmental risk assessment of RNAi-based genetically modified (GM) plants. The report is based on a systematic literature search on the use of RNAi molecules in arthropods, nematodes, annelids and molluscs with dsRNA, siRNA and miRNA applied primarily through feeding and soaking (oral ingestion). The numbers of retrieved publications covering these areas are reported, along with the species name, life stages tested, the target gene and its function, details of the test substances and their concentrations used, methods of delivery and effects. Separate sections discuss the available information on: (1) the uptake and systematic spread of RNAi activity, including a description of the various components involved in this process; (2) the mechanisms of dsRNA-, siRNA- and miRNA-elicited gene silencing and the different factors involved in RNAi efficiency; (3) routes of exposure of the biotic and abiotic environment to dsRNA, siRNA and miRNA from GM plants; (4) the environmental fate of dsRNA, siRNA and miRNA; and (5) the various factors that may limit non-target effects including exposure, factors influencing the silencing efficiency of dsRNA, siRNA and miRNA, possible unintended and off-target effects, and their mechanisms. Finally, an overview of the species of arthropods, nematodes, annelids and molluscs for which genomic data are available is also presented. The report identifies some of the challenges involved in developing plants with RNAi systems which affect invertebrate gene expression. The report also concludes that, currently, knowledge on issues such as exposure, specificity, offtarget effects, sequence similarities and bioinformatics is very limited, as only a few RNAi expressing plants which specifically target invertebrate species have been developed and comprehensively studied.
\end{abstract}

(c) European Food Safety Authority, 2018

Key words: Genetically modified (GM) plants, RNA interference (RNAi), systematic literature search, invertebrates, biosafety, specificity, bioinformatics

Question number: EFSA-Q-2016-00329

Correspondence: GMO@efsa.europa.eu 
Disclaimer: The present document has been produced and adopted by the bodies identified above as author(s). This task has been carried out exclusively by the author(s) in the context of a contract between the European Food Safety Authority and the author(s), awarded following a tender procedure. The present document is published complying with the transparency principle to which the Authority is subject. It may not be considered as an output adopted by the Authority. The European Food Safety Authority reserves its rights, view and position as regards the issues addressed and the conclusions reached in the present document, without prejudice to the rights of the authors.

Acknowledgements: The authors wishes to thank EFSA staff members Fernando Álvarez, Yann Devos, Irene Muñoz Guajardo and Nikoletta Papadopoulou for the support provided to this scientific output.

Suggested citation: Christiaens O, Dzhambazova T, Kostov K, Arpaia S, Joga MR, Urru I, Sweet J, Smagghe G, 2018. Literature review of baseline information on RNAi to support the environmental risk assessment of RNAi-based GM plants. EFSA supporting publication 2018:EN-1424. 173 pp. doi:10.2903/sp.efsa.2018.EN-1424

ISSN: $2397-8325$

(C) European Food Safety Authority, 2018

Reproduction is authorised provided the source is acknowledged.

The present document has been produced and adopted by the bodies identified above as authors. This task has been carried out exclusively by the author(s) in the context of a contract between the European Food Safety Authority and the authors, awarded following a tender procedure. The present document is published complying with the transparency principle to which the Authority is subject. It may not be considered as an output adopted by the Authority. The European Food Safety Authority reserves its rights, view and position as regards the issues addressed and the conclusions reached in the present document, without prejudice to the rights of the authors. 


\section{Summary}

A systematic literature search was used for a review of the scientific information on RNA interference (RNAi) that supports the environmental risk assessment (ERA) of RNAi-based genetically modified (GM) plants. The process involved identifying and retrieving scientific evidence for the review considering the following major aspects: definition of the review questions; identification of the information sources which are likely to yield relevant studies; definition of the keywords and search strings for identifying relevant studies; strategy for management of the references and the documents retrieved; documentation and reporting of the searches; definition of the inclusion and exclusion criteria; selection procedure for relevant studies. Web of Science (WoS) and CAB were the main information sources used. The search strings were tested against a reference collection of relevant articles and refined to minimise the number of irrelevant studies. The literature searches in online databases WoS and CAB Abstracts resulted in a total number of 17,680 records, which were reduced to 13,867 unique studies after removing duplicates, books, book sections and patents. The initial screening of these 13,867 records/studies revealed eight major methods for delivery of interfering RNA molecules in around 300 invertebrate species belonging to annelids, nematodes, arthropods and molluscs.

By far the most information on the mechanisms and pathways for RNAi is available in nematodes, and more specifically, in the free-living Caenorhabditis elegans. This nematode possesses a high sensitivity for feeding RNAi, due to a well-developed system for cellular uptake, systemic spread and an amplification system which produces secondary small interfering RNAs (siRNAs). Furthermore, the silencing signal in $C$. elegans can be observed over multiple generations. However, as has been clearly shown in the literature, a considerable degree of variation exists within nematodes concerning these pathways. Most nematodes outside of the genus of Caenorhabditis are missing several genes which are considered important in $C$. elegans, which may explain observed differences in RNAi efficiency in different nematode species. While there are other factors involved in explaining variability in RNAi sensitivity in invertebrates, cellular uptake of the small RNA (sRNA) and systemic spread of the silencing signal have been shown to be critical mechanisms for environmental RNAi.

More differences and variability in terms of uptake and systemic spread pathways were found in arthropods, where it seems that cellular uptake is much less efficient in some arthropods, especially some insect orders such as Diptera and Lepidoptera. While arthropods contain some elements of the elaborate cellular uptake and systemic spread systems found in $C$. elegans, their requirement and involvement in successful environmental or systemic RNAi has not yet been clearly demonstrated. In insects, and possibly some other taxonomic groups, cellular uptake is regulated by two pathways which may or may not be linked. One of these pathways involves the sid-1-like gene found in insect genomes, the other is based on clathrin-mediated endocytosis. The occurrence and robustness of systemic activity of RNAi in arthropods is variable and no evidence for an amplification system of the silencing signal, as is present in $C$. elegans and many other nematodes, has been reported yet.

Of the 4,612 studies which included all oral intake and soaking exposure, only 122 studies were considered relevant for the review of environmental exposure and fate. In addition, a few studies that were published after the completion of the literature search were also considered relevant and five additional studies in which transgenerational transmission of interference was studied in vitro via injection of RNAi, were also selected as relevant for this task. The analysis of the studies reporting GM plants transformed with double-stranded RNA (dsRNA) indicates that, for the majority of the cases, information on dsRNA expression is insufficient to run a robust exposure analysis and in several of these studies, detection of dsRNA in plants was not conducted. When the results of dsRNA detection in plants are reported, relative values of expression compared to reference genes are indicated. Similarly, only occasionally were expression levels detailed in different plant tissues or different phenological stages of the plant life cycle. Studies of in planta relative expression of dsRNA compared to housekeeping genes do not indicate actual amounts of dsRNA detected in plants, therefore they only orientate qualitatively an exposure analysis. By contrast, in the studies of the

The present document has been produced and adopted by the bodies identified above as authors. This task has been carried out exclusively by the author(s) in the context of a contract between the European Food Safety Authority and the authors, awarded following a tender procedure. The present document is published complying with the transparency principle to which the Authority is subject. It may not be considered as an output adopted by the Authority. The European Food Safety Authority reserves its rights, view and position as regards the issues addressed and the conclusions reached in the present document, without prejudice to the rights of the authors. 
characterization of GM maize MON 87411, a quantitative determination of dsRNA expression in various plant parts was conducted using a commercial hybridization-based molecular kit, which allows sensitive detection of dsRNA in all plant tissues.

From the relatively small amount of data on expression of dsRNA in plants, it can be inferred that in different transformation events with dsRNA targeting different genes, expression in plant parts was variable and the highest expression was detected in different tissues (from leaves to flowers) in different studies. Therefore, it will be necessary to characterize the expression levels in each GM event in order to determine exposure levels of both target organisms (TO) and non-target organisms (NTO).

Environmental persistence of dsRNA is mostly limited, as laboratory studies found dsRNA to be mostly degraded within 48 hours in soil and aquatic environments. However, in a few cases low levels of dsRNA could be detected for several days longer. Movement of dsRNA along trophic chains and the persistence of its biological activity have been shown in a few multi-trophic systems. The likelihood of a biological effect is primarily linked to the uptake of dsRNA in taxonomically different organisms and the efficiency of RNAi in the exposed organism. Similarly, intergenerational effects have been studied in a handful of cases where exogenous dsRNA was observed in a number of generations after exposure.

Reports on studies of RNAi sensitivity and efficiency in invertebrates were reviewed, mainly focusing on feeding RNAi. Several influencing factors, for example stability of dsRNA in the invertebrate body, the cellular uptake of dsRNA from the gut, the RNAi core machinery and the possible effect of viral infections, have been identified by various authors. The reports show that sensitivity and efficiency is very variable, not only between species, but also sometimes between strains, (laboratory) cultures, life stages or due to experimental aspects such as the sRNA molecule that is used. While some of these factors, such as dsRNA stability in the insect body and cellular uptake, have attracted a lot of attention from researchers, a number of other factors, such as the influence of the life stage or the impact of viral infections have not been studied in depth.

The systematic search identified 42 studies of target specificity, off-target effects and/or effects on non-target invertebrates, linked to interference triggered by dsRNA. However, there were few indepth studies of off-target and non-target activity of ingested dsRNA particularly from GM plant sources. There were some reports of silencing effects in the presence of mismatches between sequences and non-specific silencing which indicate that the accurate design of the dsRNA to induce interference does not exclude the possibility of off-target and/or non-target effects. The support of bioinformatics is identified as being valuable, but the limited availability of invertebrate genomic sequences, the possible silencing in presence of mismatches between the target and the siRNA sequences, and the possibility of sequence-unrelated off-target effects indicate the requirement for bioassays in assessing the actual activity spectrum of dsRNA.

An overview of the available genomic data (in February 2017) for invertebrates belonging to the phyla of nematodes, arthropods, molluscs and annelids in four major databases or platforms is presented. Although studies indicated that successful gene silencing requires a high degree of homology, there is no consensus yet on exact 'rules' for siRNA/RISC binding to the homologous messenger RNA. Therefore, relying only on the use of bioinformatics to predict silencing effects in targets, off-targets and non-target invertebrates is difficult at present. Furthermore, there is no clear evidence for the number of siRNAs, processed from long dsRNA for example, necessary to incite gene silencing. Therefore, genomic data alone is not guaranteed to predict absence of silencing effects. Additionally, one must consider that genome sequences never have a $100 \%$ coverage of the genome, nor are they always $100 \%$ accurate.

The report concludes by highlighting the gaps in our baseline knowledge that warrant further research in order to support environmental risk assessments.

The present document has been produced and adopted by the bodies identified above as authors. This task has been carried out exclusively by the author(s) in the context of a contract between the European Food Safety Authority and the authors, awarded following a tender procedure. The present document is published complying with the transparency principle to which the Authority is subject. It may not be considered as an output adopted by the Authority. The European Food Safety Authority reserves its rights, view and position as regards the issues addressed and the conclusions reached in the present document, without prejudice to the rights of the authors. 


\section{Table of contents}

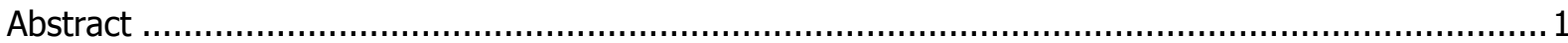

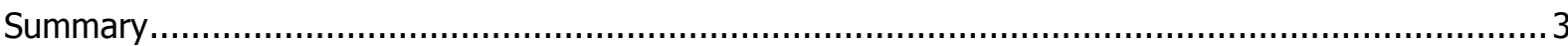

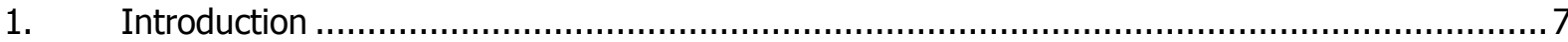

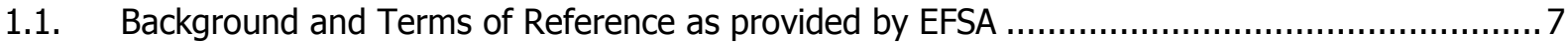

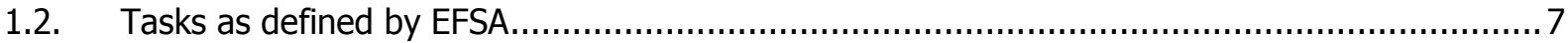

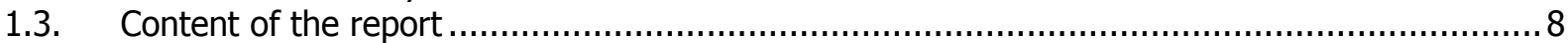

1.4. Introduction to RNAi-based GM plants for invertebrate control ..................................... 10

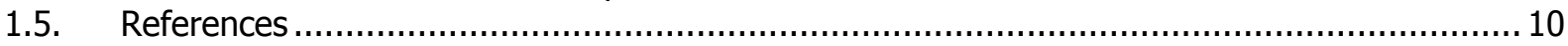

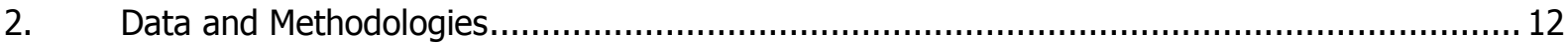

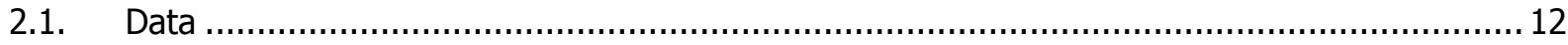

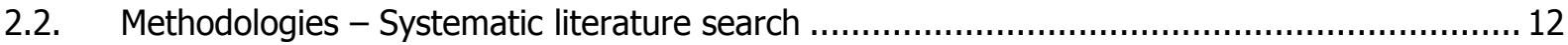

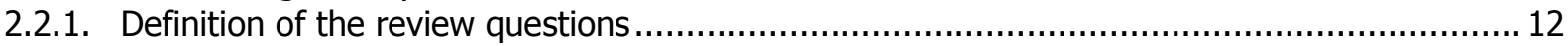

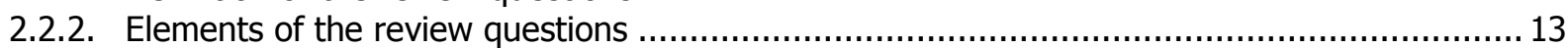

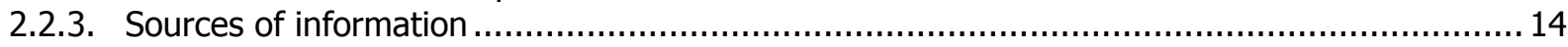

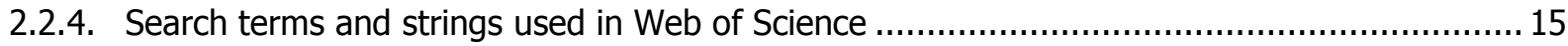

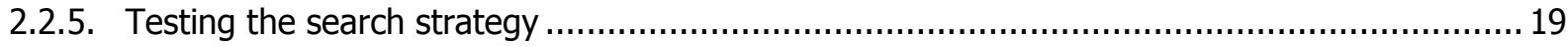

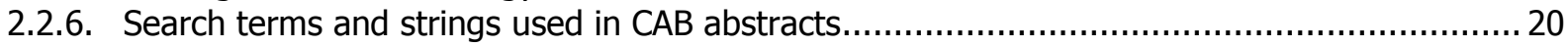

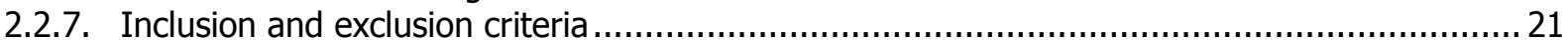

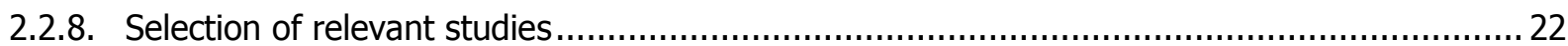

2.2.9. Relevance assessment criteria for studies on environmental exposure (Task 3) and off-target,

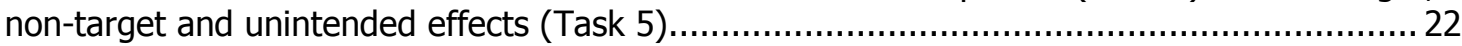

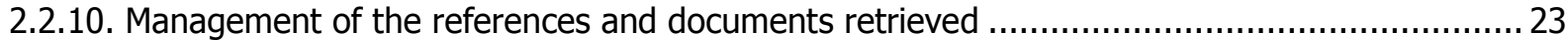

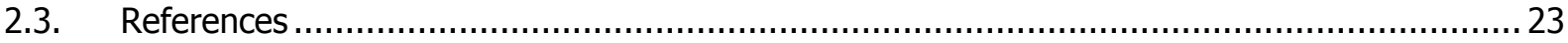

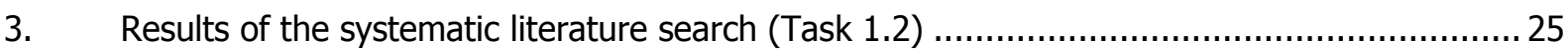

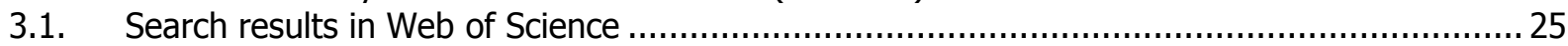

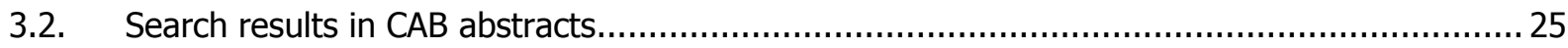

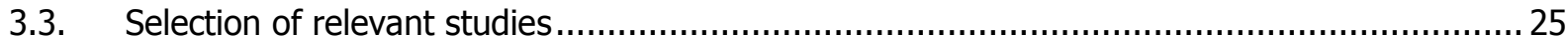

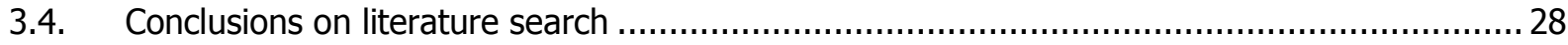

4. Analysis of the primary data from the selected studies ................................................ 30

4.1. Activity spectrum of the tested dsRNAs, siRNAs and miRNAs in invertebrates (Task 1.3) .......30

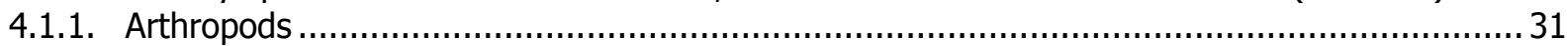

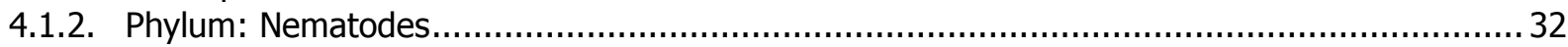

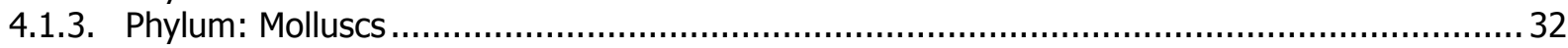

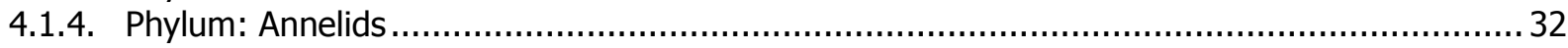

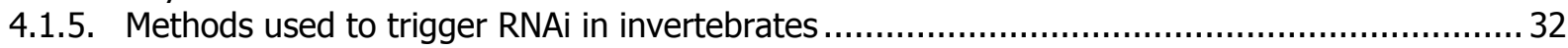

4.2. Oral introduction of siRNA and miRNA and the induction of efficient RNAi in arthropods,

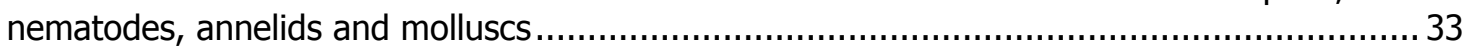

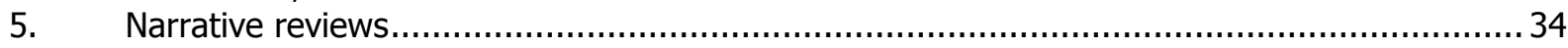

5.1. Uptake and systemic spread of small RNAs in invertebrates (Task 2) ................................. 34

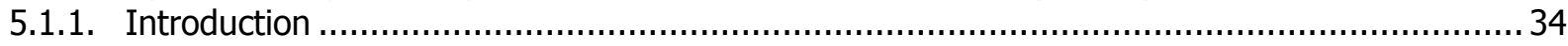

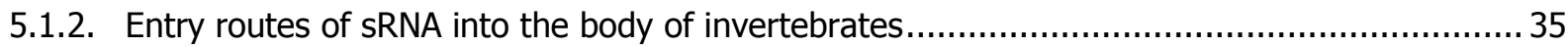

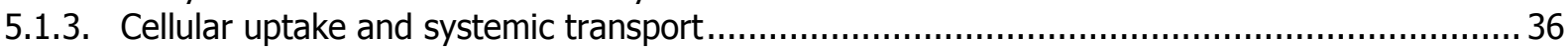

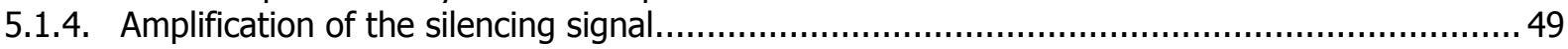

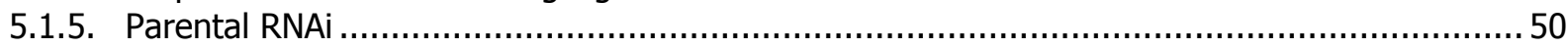

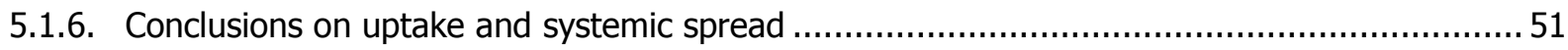

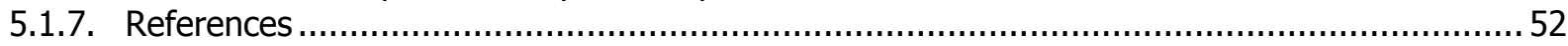

5.2. Environmental exposure and fate of dsRNA, siRNA and miRNA (Task 3)..........................63

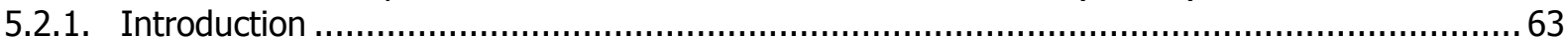
by the author(s) in the context of a contract between the European Food Safety Authority and the authors, awarded following a tender procedure. The present document is published complying with the transparency principle to which the Authority is subject. It may not be considered as an output adopted by the Authority. The European Food Safety Authority reserves its rights, view and position as regards the issues addressed and the conclusions reached in the present document, without prejudice to the rights of the authors. 
5.2.2. Environmental exposure routes from plants to invertebrates ...........................................63

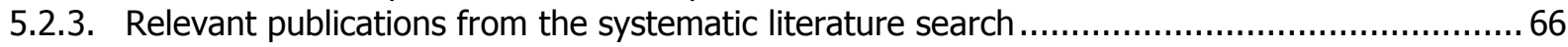

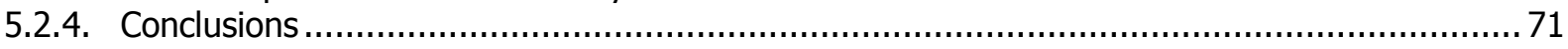

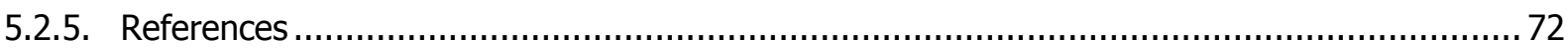

5.3. Factors influencing silencing efficiency of dsRNA, siRNA and miRNA delivered orally in arthropods, nematodes, annelids and molluscs (Task 4) .................................................. 78

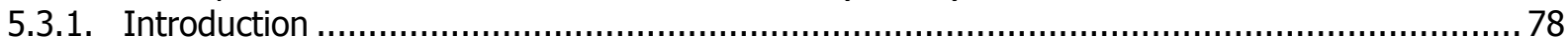

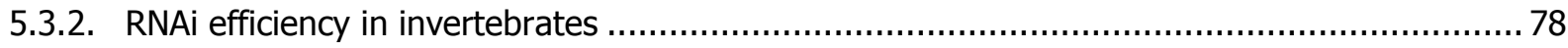

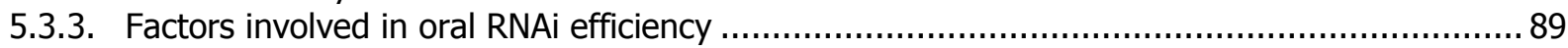

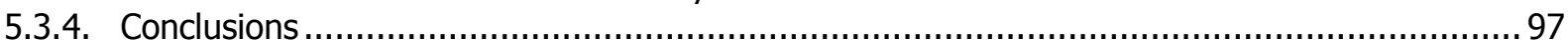

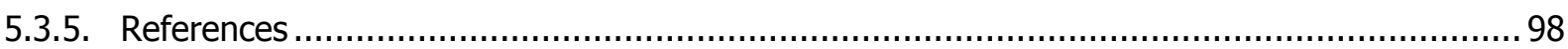

5.4. Off-target, non-target and unintended effects of RNAi-based GM plants (Task 5) ............... 114

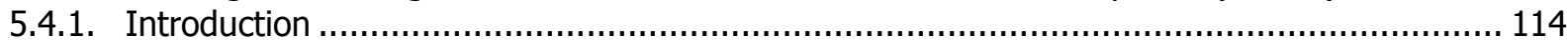

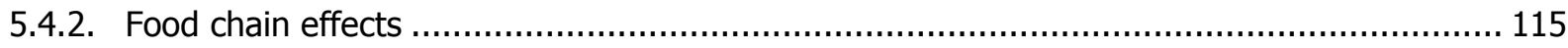

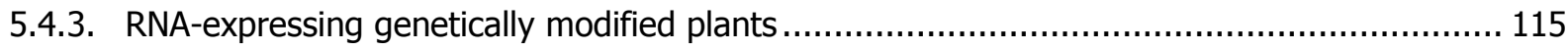

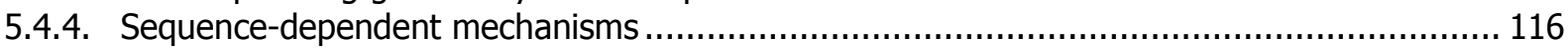

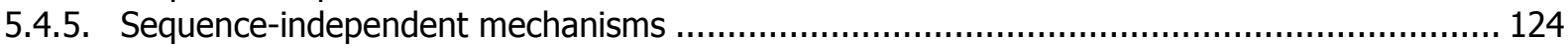

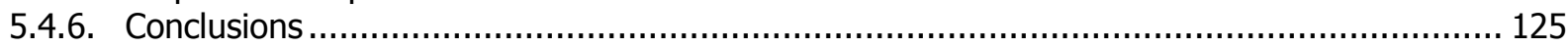

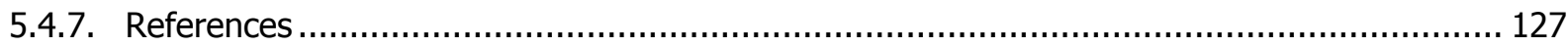

5.5. Overview of invertebrate species for which a sequenced genome is available (Task 6)........ 132

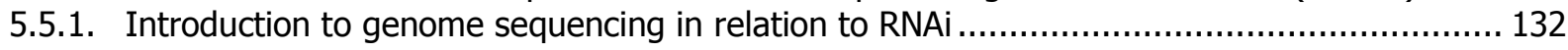

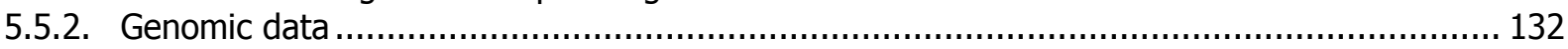

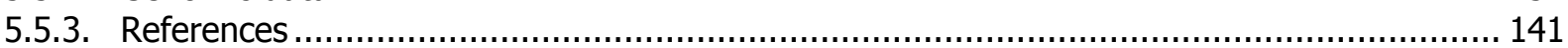

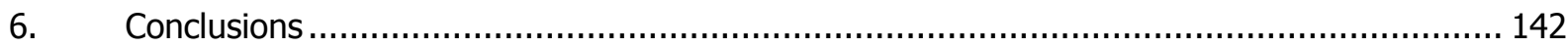

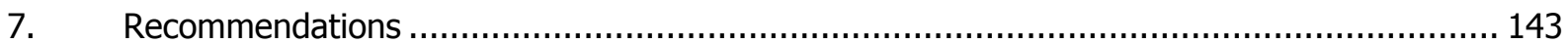

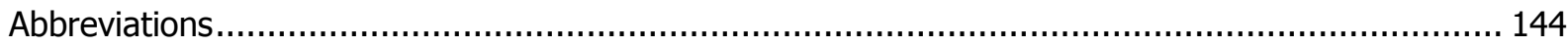

Appendix A - List of reference studies used to assess the relevance of the results from the defined

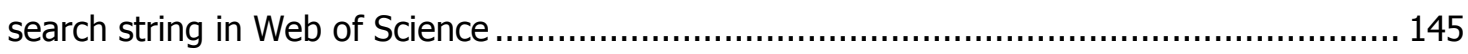

Appendix B - List of invertebrate species or genera used in RNAi studies ............................... 152

Appendix C - List of publications considered relevant for Task 3 "Environmental exposure and fate of dsRNA siRNA and miRNA" - Plant expression and intergenerational transmission............ 161

Appendix D - $\quad$ List of publications considered relevant for Task 3 "Environmental exposure and fate of dsRNA siRNA and miRNA" - Environmental fate........................................................ 168

Appendix E - List of publications deemed relevant for Task 5 "Off target, non-target and unintended effects of RNAi-based GM plants" ............................................................ 170 by the author(s) in the context of a contract between the European Food Safety Authority and the authors, awarded following a tender procedure. The present document is published complying with the transparency principle to which the Authority is subject. It may not be considered as an output adopted by the Authority. The European Food Safety Authority reserves its rights, view and position as regards the issues addressed and the conclusions reached in the present document, without prejudice to the rights of the authors. 


\section{Introduction}

\subsection{Background and Terms of Reference as provided by EFSA}

This report is an outcome of an EFSA procurement titled "Literature review of baseline information to support the risk assessment of RNAi-based GM plants-lot 2" (reference number OC/EFSA/GMO/2015/02), awarded by the European Food Safety Authority to Ghent University, in consortium with Agrobioinstitute (ABI), Italian National Agency for New Technologies, Energy and Sustainable Economic Development (ENEA), and JT Environmental Consultants Ltd.

\subsection{Tasks as defined by EFSA}

The following tasks were provided by EFSA in the tender specifications:

Task 1: To perform a systematic literature search, so as to provide an overview on the use of host-delivered RNAi molecules in arthropods, nematodes, annelids and molluscs with double stranded, small interfering and micro RNAs (dsRNAs, siRNAs and miRNAs, respectively) applied through feeding (oral ingestion).

The following relevant factors should be reported per experimental study published in the peer-reviewed scientific literature: (1) Test organism: taxonomy (phylum, order, family, and species name), functional group, life stage tested, number of organisms tested, number of replications per treatment, number of generations tested (if appropriate); (2) target gene and its function as well as the gene location in the recipient organism; (3) test substance (purified dsRNA, siRNA and miRNA), small RNA expressing bacteria or genetically modified (GM) plant tissue) and method of delivery (e.g., artificial diet, GM plant tissue); (4) test concentrations (or expression levels); (5) length of dsRNA, siRNA and miRNA; (6) test duration/duration of exposure; (7) measurement endpoints followed (lethal and sublethal); (8) controls used (negative control, positive control); (9) observed effects in terms of messenger RNA (mRNA) silencing, including the amount of silencing (relative expression of the target gene compared to the control), affected measurement endpoints, the recovery of gene expression, and evaluation method; (10) any other additional information considered relevant (e.g., statistical power); (11) the reference of the scientific publication.

- Task 1.1: To report on the approach followed and the outcomes of the systematic literature search performed to identify and retrieve relevant publications published in the peer-reviewed scientific literature (e.g., search terms/string, scientific literature databases, inclusion/exclusion criteria for the identification of relevant scientific publications).

- Task 1.2: To report on the outcomes of the systematic literature search in terms of relevant scientific publications identified and retrieved.

- Task 1.3: Based on the retrieved information, to summarise the activity spectrum of the tested dsRNAs, siRNAs and miRNAs.

- Task 1.4: Based on the retrieved information, to assess whether, and if so under which conditions, siRNA and miRNA delivered to arthropods, nematodes, annelids and molluscs through feeding trigger (efficient) RNAi.

- Task 2: Based on available scientific literature, to review mechanisms of dsRNA (and siRNA and miRNA, if relevant) uptake and systematic spread in arthropods, nematodes, annelids and molluscs, including a description of the various components involved in this process (narrative review).

- Task3: Based on available scientific literature, to review plausible routes of exposure of the biotic and abiotic environment to dsRNA (and siRNA and miRNA, if relevant) expressed in RNAibased GM plants, the environmental fate of dsRNA (and siRNA and miRNA, if relevant), and the various factors that may limit non-target organism (NTO) exposure (barriers to exposure:

The present document has been produced and adopted by the bodies identified above as authors. This task has been carried out exclusively by the author(s) in the context of a contract between the European Food Safety Authority and the authors, awarded following a tender procedure. The present document is published complying with the transparency principle to which the Authority is subject. It may not be considered as an output adopted by the Authority. The European Food Safety Authority reserves its rights, view and position as regards the issues addressed and the conclusions reached in the present document, without prejudice to the rights of the authors. 
e.g., enzymatic barriers, $\mathrm{pH}$ in gut of recipient organisms, (in)stability of RNAi molecules in recipient organisms) (narrative review).

- Task 4: Based on available scientific literature and the information retrieved in the previous tasks, to summarise which factors largely influence silencing efficiency of dsRNA (and siRNA and miRNA, if relevant) across arthropods, nematodes, annelids and molluscs delivered through feeding (narrative review).

- Task 5: Based on available scientific literature, to assess the plausibility of unintended adverse effects on arthropods, nematodes, annelids and molluscs associated with the cultivation of RNAi-based GM plants (covering lethal, sublethal and chronic effects in the various life stage of NTOs), and involved mechanisms (e.g., unintended gene suppression, immunostimulation, saturation of RNAi machinery, potential for resistance evolution in target pests) (narrative review).

- Task 6: To provide an overview of species (including model species) belonging to the taxa of arthropods, nematodes, annelids and molluscs for which complete or partial genome data are available (narrative review).

\subsection{Content of the report}

Section 1 presents the tasks defined by EFSA (Section 1.2) and summarises the content of the report (Section 1.3). It also includes a general introductory section (Section 1.4), providing some background information on RNA silencing and its development for control of invertebrate pests. Detailed information on the mechanisms and functions of RNA silencing in a range of organisms including some invertebrates is provided in the report on the parallel lot "Literature review of baseline information to support the risk assessment of RNAi-based GM plants - lot 1" (reference number OC/EFSA/GMO/2015/01), awarded by EFSA to the Institute of Molecular Genetics of the Academy of Sciences of the Czech Republic (IMG) in consortium with EcoMole Ltd. (Pačes et al., 2017)

- Section 2 describes the methodology used to perform the systematic literature search (Task 1).

- Section 3 describes the results of the systematic literature search for baseline information to support the environmental risk assessment (ERA) of RNAi-based GM plants (Tasks 1.1 and 1.2).

- Section 4 analyses the primary data from the selected studies, and describes the activity spectrum of the tested dsRNAs, siRNAs and miRNAs (Task 1.3), based on the relevant studies retrieved from the systematic literature search. Scientific literature on invertebrate species (i.e., arthropods, nematodes, annelids and molluscs) studied and the range of genes targeted by RNAi systems is reported, and the activity spectrum of the target gene that is altered described, including the impact on the physiology and metabolism of the target (and nontarget) organism and its ultimate demise (e.g., loss of fertility, mortality). The specificity of each dsRNA, siRNA and miRNA is described in terms of its activity spectra within the different tissues, and also the range activities in non-target species. This section also assesses whether, and if so under which conditions, siRNA and miRNA delivered to arthropods, nematodes, annelids and molluscs through feeding trigger (efficient) RNAi (Task 1.4). Studies of soaking and oral introduction of siRNA and miRNA into invertebrates are reviewed for the methods, dose and longevity of feeding exposure, results and outcomes of each study. This part discusses which systems provide sufficient siRNA and miRNA to trigger efficient RNAi and whether they also persist or stimulate multiplication/replication within these invertebrates. It also considers their activity spectra within the different tissues of an organism and also the range of off-target activities and non-target effects. The implications of the efficiency of oral

The present document has been produced and adopted by the bodies identified above as authors. This task has been carried out exclusively by the author(s) in the context of a contract between the European Food Safety Authority and the authors, awarded following a tender procedure. The present document is published complying with the transparency principle to which the Authority is subject. It may not be considered as an output adopted by the Authority. The European Food Safety Authority reserves its rights, view and position as regards the issues addressed and the conclusions reached in the present document, without prejudice to the rights of the authors. 
RNAi on resistance development, non-specific/off-target effects and non-target effects are discussed.

- Section 5 contains the narrative literature reviews, as defined by EFSA (Tasks 2-5):

- Section 5.1: Uptake and systemic spread of small RNAs in invertebrates (Task 2). This section includes a study of entry routes of SRNA into the body of invertebrates and cellular uptake and systemic transport and the influence of the small RNA (sRNA) molecule on cellular uptake and systemic RNAi efficiency. The literature on nematodes is discussed with a focus on cellular uptake, transport and systemic RNAi efficiency in Caenorhabditis elegans, as this is one of the most intensively studied species, and this is contrasted with information from studies with other nematode species, including plant parasitic species. The literature on other invertebrates is discussed with a focus on cellular uptake, transport and systemic RNAi efficiency in arthropods where insects such as Drosophila have been extensively studied, and in annelids and molluscs. Reports of amplification of the silencing signal and transgenerational activity of parental RNA are reviewed.

- Section 5.2: Environmental exposure and fate of dsRNA, siRNA and miRNA (Task 3). In this section, the information on exposure routes and pathways and the fate of dsRNA and siRNA is discussed and considered in relation to possible exposure routes to invertebrates and the evaluation of exposure for risk assessment purposes. Data on persistence of dsRNA in plant and animal material, soil and soil water is examined. Exposure is also an important element in resistance development in target organisms so that the exposure information also informs the studies of resistance discussed in Section 5.4 (Task 5).

- Section 5.3: Factors influencing silencing efficiency of dsRNA, SiRNA and miRNA delivered orally in arthropods, nematodes, annelids and molluscs (Task 4). In this section studies related to the silencing efficiency and efficacy of dsRNA, siRNA and miRNA in this range of invertebrates are described and discussed. The factors involved in restricting and promoting the efficiency of RNAi in invertebrates are identified and examined, both at the molecular mechanism level and in relation to the uptake studies in Section 5.1 (Task 2) and the exposure studies in Section 5.2 (Task 3). This section considers the published information relating to these factors and discusses the evidence that feeding studies of the different RNAs are resulting in effective RNAi of target invertebrate species. The factors affecting the success or failure of systems are analysed, and influences of these factors on non-target and off target effects discussed.

- Section 5.4: Off-target, non-target and unintended effects of RNAi-based GM plants (Task 5). This section reviews the studies on target specificity, off-target effects and/or effects on non-target invertebrates, linked to interference triggered by dsRNA. It focuses on studies of off-target and non-target activity of ingested dsRNA from GM plant sources. It considers reports of silencing effects in presence of mismatches between sequences and non-specific silencing. It examines whether the accurate design of the dsRNA to induce interference excludes the possibility of off-target and/or non-target effects. The possible silencing in presence of mismatches between the target and the siRNA sequences is considered, and the possibility of sequence-unrelated off-target effects are discussed. In addition, the mechanisms and potential for resistance evolution in target pests is considered.

- Section 5.5: Genomic data on arthropods, nematodes, annelids and molluscs (Task 6). This section gives an overview of the available genomic data for arthropods, nematodes, annelids and molluscs in four major databases or platforms. The potential use of bioinformatics to predict silencing effects in targets, off-targets and non-target

The present document has been produced and adopted by the bodies identified above as authors. This task has been carried out exclusively by the author(s) in the context of a contract between the European Food Safety Authority and the authors, awarded following a tender procedure. The present document is published complying with the transparency principle to which the Authority is subject. It may not be considered as an output adopted by the Authority. The European Food Safety Authority reserves its rights, view and position as regards the issues addressed and the conclusions reached in the present document, without prejudice to the rights of the authors. 
invertebrates is considered in relation to the 'rules' for binding of siRNA, which is incorporated in the RNA induced silencing complex (RISC), to the homologous mRNA.

- Section 6 summarises the main findings and conclusions from each section and identify gaps in data and knowledge, whereas in Section $\mathbf{7}$ further studies are recommended to provide more information on areas where knowledge and experience is limited, in order to support the ERA of GM-based RNAi plants.

\subsection{Introduction to RNAi-based GM plants for invertebrate control}

RNAi was discovered in 1998 in the nematode $C$. elegans, where injection of dsRNA caused a specific post-transcriptional gene silencing (Fire et al., 1998). Soon after, it was shown that this mechanism is conserved in most Eukaryota, including insects. When it became clear that oral delivery of dsRNA was also able to elicit RNAi, scientists soon realised the potential of this technique as a pest control strategy. This has encouraged research on developing plants that could be protected from herbivorous pests by engineering them to express dsRNAs targeting vital genes in the pest. In recent years, there have been several studies of this approach further demonstrating the potential of this mechanism for crop protection. The modes of action and mechanisms associated with sRNAs in a range of animal and plant species have been the subject of another review commissioned by EFSA (Pačes et al., 2017). However, the variable efficiency of RNAi in many arthropods is a challenge that must be addressed before RNAi can be widely used in pest control (Terenius et al., 2011; Christiaens and Smagghe, 2014). In this report, we use the term sRNAs to refer to miRNA, siRNA and longer dsRNA (25-1500 bp).

Mao et al. (2007), working with cotton bollworm (Helicoverpa zea), and Baum et al. (2007), working with a range of insects that includes western corn rootworm (WCR, Diabrotica virgifera virgifera), southern corn rootworm (SCR, Diabrotica undecimpunctata howardi) and Colorado potato beetle (CPB, Leptinotarsa decemlineata) have shown that transgene-encoded ingestible dsRNA can specifically target certain pest insect genes and prevent their function, resulting in mortality of the pest. Since then, a number of studies with other arthropod species have demonstrated similar effects with ingested dsRNAs, and there are studies also with other sRNAs such as siRNAs and miRNAs (Zotti and Smagghe, 2015). In 2013, the US Environmental Protection Agency (US EPA) approved an experimental use permit (No. 524-EUP-104) for field evaluation of a GM maize expressing a transgene construct which includes the Snf7 gene which suppresses mRNA encoding vacuolar ATPase subunits A and E. This gene targets the WCR, and published data indicate the specificity of the Snf7 gene to the target pest ATPase mRNAs (Bachman et al., 2013). This has raised a number of issues relating to the risk assessment of RNAi plants as indicated by Ramon et al. (2014), Casacuberta et al. (2015) and Zotti and Smagghe (2015), and the need to clearly identify all the available information that can underpin and support ERA of these GM-based RNAi plants.

\subsection{References}

Bachman PM, Bolognesi R, Moar WJ, Mueller GM, Paradise MS, Ramaseshadri P, Tan J, Uffman JP, Warren J, Wiggins BE and Levine SL, 2013. Characterization of the spectrum of insecticidal activity of a double-stranded RNA with targeted activity against Western Corn Rootworm (Diabrotica virgifera virgifera LeConte). Transgenic Research, 22, 1207-1222.

Baum JA, Bogaert T, Clinton W, Heck GR, Feldmann P, Ilagan O, Johnson S, Plaetinck G, Munyikwa T, Pleau M, Vaughn T and Roberts J, 2007. Control of coleopteran insect pests through RNA interference. Nature Biotechnology, 25, 1322-1326.

Casacuberta JM, Devos Y, du Jardin P, Ramon M, Vaucheret H and Nogué F, 2015. Biotechnological uses of RNAi in plants: risk assessment considerations. Trends in Biotechnology, 33, 145-147. procedure. The present document is published complying with the transparency principle to which the Authority is subject. It may not be considered as an output adopted by the Authority. The European Food Safety Authority reserves its rights, view and position as regards the issues addressed and the conclusions reached in the present document, without prejudice to the rights of the authors. 
Christiaens O and Smagghe G, 2014. The challenge of RNAi-mediated control of hemipterans. Current Opinion in Insect Science, 6, 15-21.

Fire, A, Xu, SQ, Montgomery, MK, Kostas, SA, Driver, SE, Mello, CC, 1998. Potent and specific genetic interference by double-stranded RNA in Caenorhabditis elegans. Nature, 391, 806-811.

Mao YB, Cai WJ, Wang JW, Hong GJ, Tao XY,Wang L, Huang YP and Chen XY, 2007. Silencing a cotton bollworm $\mathrm{P} 450$ monooxygenase gene by plant mediated RNAi impairs larval tolerance of gossypol. Nature Biotechnology, 25, $1307-1313$.

Pačes J, Nič M, Novotný T and Svoboda P, 2016. Literature review of baseline information to support the risk assessment of RNAi-based GM plants. EFSA supporting publication, 14, doi:10.2903/sp.efsa.2017.EN-1246

Ramon M, Devos Y, Lanzoni A, Liu Y, Gomes A, Gennaro A and Waigmann E, 2014. RNAi-based GM plants: food for thought for risk assessors. Plant Biotechnology Journal, 12, 1271-1273.

Terenius et al., 2011. RNA interference in Lepidoptera: An overview of successful studies and implications for experimental design. Journal of Insect Physiology, 57, 231-245.

Zotti MJ and Smagghe G, 2015. RNAi technology for insect management and protection of beneficial insects from diseases. Neotrophic Entomology, 44, 197-213. by the author(s) in the context of a contract between the European Food Safety Authority and the authors, awarded following a tender procedure. The present document is published complying with the transparency principle to which the Authority is subject. It may not be considered as an output adopted by the Authority. The European Food Safety Authority reserves its rights, view and position as regards the issues addressed and the conclusions reached in the present document, without prejudice to the rights of the authors. 


\section{Data and Methodologies}

\subsection{Data}

Data was obtained from available scientific literature and from relevant publications retrieved from the systematic literature search.

\subsection{Methodologies - Systematic literature search}

A systematic literature search was performed to obtain/retrieve relevant studies/information to support the ERA of RNAi-based GM plants. The process towards identifying and retrieving scientific evidence for the review involves the following major steps:

- Definition of the review questions;

- Identification of the information sources which are likely to yield relevant studies;

- Definition of the keywords and search strings for identifying relevant studies;

- Strategy for management the references and the documents retrieved;

- Documentation and reporting of the searches;

- Definition of the inclusion and exclusion criteria;

- Selection procedure for relevant studies.

\subsubsection{Definition of the review questions}

The team defined five review questions which aim to address the topics described in the tender specifications, as follows:

- Activity spectrum and conditions for efficient RNAi through feeding (oral ingestion) of arthropods, nematodes, annelids and molluscs with dsRNAs, siRNAs and miRNAs;

- Mechanisms of dsRNA, siRNA and miRNA uptake and systematic spread in arthropods, nematodes, annelids and molluscs, including a description of the various components involved in this process;

- Plausible routes of exposure of the biotic and abiotic environment to dsRNA, siRNA and miRNA expressed in RNAi-based GM plants, the environmental fate of dsRNA, siRNA and miRNA and the various factors that may limit NTO exposure (barriers to exposure: e.g., enzymatic barriers, $\mathrm{pH}$ in gut of recipient organisms, (in)stability of RNAi molecules in recipient organisms);

- Factors which influence silencing efficiency of dsRNA, siRNA and miRNA across arthropods, nematodes, annelids and molluscs delivered through feeding;

- Plausibility of unintended adverse effects on arthropods, nematodes, annelids and molluscs associated with the cultivation of RNAi-based GM plants (covering lethal, sublethal and chronic effects in the various life stage of NTOs), and involved mechanisms (e.g., unintended gene suppression, immunostimulation, saturation of RNAi machinery, potential for resistance evolution in target pests).

\section{Review question 1}

What is the activity spectrum and under which conditions dsRNAs, siRNAs and miRNAs delivered to arthropods, nematodes, annelids and molluscs through feeding (oral ingestion) trigger efficient RNAi?

The aim of this question is to identify the range of genes targeted by RNAi systems applied through feeding of dsRNA, siRNA and miRNA to arthropods, nematodes, annelids and molluscs. This includes the activity spectrum of the gene that is altered, the impact on the physiology and metabolism of the target (and non-target) organism and its ultimate demise (e.g., loss of fertility, mortality).

The present document has been produced and adopted by the bodies identified above as authors. This task has been carried out exclusively by the author(s) in the context of a contract between the European Food Safety Authority and the authors, awarded following a tender procedure. The present document is published complying with the transparency principle to which the Authority is subject. It may not be considered as an output adopted by the Authority. The European Food Safety Authority reserves its rights, view and position as regards the issues addressed and the conclusions reached in the present document, without prejudice to the rights of the authors. 


\section{Review question 2}

What are the components and mechanisms of dsRNA, SiRNA and miRNA uptake and systematic spread in arthropods, nematodes, annelids and molluscs?

In the focus of this question are the articles studying components and mechanisms of dsRNA, siRNA and miRNA uptake and systematic spread in invertebrates. It aims to consider specific aspects of RNAi such as the important differences in uptake and systemic transport mechanisms, the genes involved and the implications on uptake capacity of ds-, si- or miRNA molecules by arthropods, nematodes, annelids and molluscs.

\section{Review question 3}

What are the exposure routes and the environmental fate of IsRNA, SiRNA and miRNA?

This question aims at retrieving information on the various aspects of environmental exposure including exposure of soil invertebrates through plant roots, bio-pesticides (containing dsRNA), plant and animal residues, as well as studies which investigate persistence and degradation of dsRNA in the gastrointestinal tract of the invertebrates and the biological and biophysical factors driving these processes. It also includes the collection of data on persistence of dsRNA in plant and animal material and soil and the discussion of possible exposure routes to invertebrates.

\section{Review question 4}

What are the factors influencing silencing efficiency of dsRNA, siRNA and miRNA delivered orally in arthropods, nematodes, annelids and molluscs?

This question addresses one of the main topics in RNAi research at the moment related to the silencing efficiency and the observed variability between different experiments, species, delivery systems, uptake routes, etc. Getting a clear understanding of the factors that influence this efficiency and how they are linked to each other is of great importance for the development, ERA and management of this technology. This question considers the evidence that feeding studies using different RNAs are effective for RNAi of target invertebrate species. This question also examines the factors affecting the success or failure of systems and the influence of these factors on non-target and off target effects.

\section{Review question 5}

What are the off-target, non-target and unintended effects of RNAi-based GM plants to arthropods, nematodes, annelids and molluscs?

This question aims at retrieving studies which examine the off-target and non-target effects of RNAibased GM plants as well as studies of other unintended effects such as: gene suppression; immunostimulation; saturation of RNAi machinery; degradation of non-targeted mRNA by transitive RNA silencing and stability of the silenced phenotype, e.g., considering removal of toxins. The collected information is examined to determine whether RNAi systems developed in plants for silencing plant metabolic activities, or directed at other targets such as viruses, have off-target or non-target effects in invertebrates.

\subsubsection{Elements of the review questions}

The key elements of the review questions such as the population of interest, the intervention and the type of exposure were defined and used as an input for the definition of the keywords and the content of the search strings. In the next table, a description of these elements is given:

The present document has been produced and adopted by the bodies identified above as authors. This task has been carried out exclusively by the author(s) in the context of a contract between the European Food Safety Authority and the authors, awarded following a tender procedure. The present document is published complying with the transparency principle to which the Authority is subject. It may not be considered as an output adopted by the Authority. The European Food Safety Authority reserves its rights, view and position as regards the issues addressed and the conclusions reached in the present document, without prejudice to the rights of the authors. 
Table 1: Key elements of the review questions

\begin{tabular}{|c|c|c|c|}
\hline Question & Population & Intervention & Outcomes \\
\hline $\begin{array}{l}\text { What is the activity spectrum and } \\
\text { under which conditions dsRNAs, } \\
\text { siRNAs and miRNAs delivered to } \\
\text { arthropods, nematodes, annelids and } \\
\text { molluscs through feeding (oral } \\
\text { ingestion) trigger efficient RNAi }\end{array}$ & $\begin{array}{l}\text { Arthropods, } \\
\text { nematodes, annelids } \\
\text { and molluscs }\end{array}$ & $\begin{array}{l}\text { Oral delivery } \\
\text { (including soaking) } \\
\text { of dsRNA, siRNA } \\
\text { and miRNA }\end{array}$ & $\begin{array}{l}\text { Silencing or knockdown of } \\
\text { gene expression in target } \\
\text { and non-target } \\
\text { populations }\end{array}$ \\
\hline $\begin{array}{l}\text { What are the components and } \\
\text { mechanisms of dsRNA, siRNA and } \\
\text { miRNA uptake and systematic spread } \\
\text { in arthropods, nematodes, annelids } \\
\text { and molluscs? }\end{array}$ & $\begin{array}{l}\text { Arthropods, } \\
\text { nematodes, annelids } \\
\text { and molluscs }\end{array}$ & $\begin{array}{l}\text { Any type of } \\
\text { exposure in } \\
\text { laboratory or field } \\
\text { conditions (oral, } \\
\text { injection, soaking, } \\
\text { etc.) to dsRNA, } \\
\text { siRNA and miRNA }\end{array}$ & $\begin{array}{l}\text { Silencing or knockdown of } \\
\text { gene expression in target } \\
\text { and non-target } \\
\text { populations }\end{array}$ \\
\hline $\begin{array}{l}\text { What are the exposure routes and } \\
\text { the environmental fate of } d s R N A \text {, } \\
\text { siRNA and miRNA? }\end{array}$ & $\begin{array}{l}\text { Arthropods, } \\
\text { nematodes, annelids, } \\
\text { molluscs and } \\
\text { different } \\
\text { environments }\end{array}$ & $\begin{array}{l}\text { Any type of } \\
\text { exposure in } \\
\text { laboratory or field } \\
\text { conditions (oral, } \\
\text { injection, soaking, } \\
\text { etc.) to dsRNA, } \\
\text { siRNA and miRNA }\end{array}$ & $\begin{array}{l}\text { Silencing or knockdown of } \\
\text { gene expression in target } \\
\text { and non-target } \\
\text { populations }\end{array}$ \\
\hline $\begin{array}{l}\text { What are the factors influencing } \\
\text { silencing efficiency of dsRNA, siRNA } \\
\text { and miRNA delivered orally in } \\
\text { arthropods, nematodes, annelids and } \\
\text { molluscs? }\end{array}$ & $\begin{array}{l}\text { Arthropods, } \\
\text { nematodes, annelids, } \\
\text { molluscs }\end{array}$ & $\begin{array}{l}\text { Oral delivery } \\
\text { (including soaking) } \\
\text { of dsRNA, siRNA } \\
\text { and miRNA }\end{array}$ & $\begin{array}{l}\text { Silencing or knockdown of } \\
\text { gene expression in target } \\
\text { and non-target } \\
\text { populations }\end{array}$ \\
\hline $\begin{array}{l}\text { What are the target, off-target, non- } \\
\text { target and unintended effects of } \\
\text { RNAi-based GM plants in arthropods, } \\
\text { nematodes, annelids and molluscs? }\end{array}$ & $\begin{array}{l}\text { Arthropods, } \\
\text { nematodes, annelids, } \\
\text { molluscs }\end{array}$ & $\begin{array}{l}\text { GM plants and } \\
\text { other types of } \\
\text { exposure in } \\
\text { laboratory or field } \\
\text { conditions (oral, } \\
\text { injection, soaking, } \\
\text { etc.) to dsRNA, } \\
\text { siRNA and miRNA }\end{array}$ & $\begin{array}{l}\text { Silencing or knockdown of } \\
\text { gene expression and any } \\
\text { unintended effects in } \\
\text { target and non-target } \\
\text { populations }\end{array}$ \\
\hline
\end{tabular}

\subsubsection{Sources of information}

The sources of information that may yield relevant scientific studies for the review topic were selected after a discussion within the team following the best practices for conducting systematic reviews described in methodological guidelines (EFSA, 2010, 2011) and systematic review protocols (Meissle et al., 2014; Sweet and Kostov, 2014).

\subsubsection{Journals, conference abstracts or proceedings in electronic bibliographic databases}

The main sources to retrieve relevant information are journals and conference abstracts or proceedings available in electronic bibliographic databases. Bibliographic databases are the most efficient way to identify an initial set of relevant scientific studies. They are usually designed to facilitate effective information retrieval with information presented in structured ways and often with the addition of indexing. Multi-disciplinary and subject-specific databases were used to allow consideration of complementarity and redundancy. It also minimised the impact of publication bias, and helped to address limitations in research reporting and indexing. The selected electronic databases include the largest abstracting literature databases, full text engines that provide

The present document has been produced and adopted by the bodies identified above as authors. This task has been carried out exclusively by the author(s) in the context of a contract between the European Food Safety Authority and the authors, awarded following a tender procedure. The present document is published complying with the transparency principle to which the Authority is subject. It may not be considered as an output adopted by the Authority. The European Food Safety Authority reserves its rights, view and position as regards the issues addressed and the conclusions reached in the present document, without prejudice to the rights of the authors. 
comprehensive lists of information sources. These databases were selected because they cover studies in all the relevant areas of the biological sciences relating to RNAi in plants and invertebrates.

The search was carried out in the following electronic bibliographic databases:

- Web of Science (WoS) (ISI Web of Knowledge 1900 - 2016) (Thomson Reuters, New York, USA), a multi-disciplinary keyword database, contains peer-reviewed scientific studies. The WoS platform conducts searches in the following databases according to the subscription of ABI:

- Web of Science ${ }^{T M}$ Core CollectionBIOSIS Citation Index ${ }^{S M}$

- Current Contents Connect

- Data Citation IndexSM

Derwent Innovations Index ${ }^{S M}$

KCI-Korean Journal Database

MEDLINE ${ }^{\circledR}$

Russian Science Citation Index

SciELO Citation Index

Zoological Record ${ }^{\circledR}$

- CAB Abstracts (1984-2016) (CABI, Wallingford, UK), comprehensive database for the applied life sciences - agriculture, environment, veterinary sciences, applied economics, food science and nutrition. This database also includes local and non-English studies.

\subsubsection{Reference lists}

A list of references was compiled, which consisted of 131 RNAi studies in invertebrates, to be used as a validation of the search string. The compilation was made based on the reference list of six recent reviews on relevant topics and methodological articles (Kumar et al., 2013; Nandety et al., 2015; Owens and Malham, 2015; Roberts et al., 2015; Schumpert et al., 2015; Zotti and Smagghe, 2015), or studies provided by the team members based on their experience in the subject.

\subsubsection{Search terms and strings used in Web of Science}

The search in WoS covered time period from 1998 until July 2016. The time span of the search started with 1998, the year when gene silencing, after introduction of dsRNA in invertebrate species (C. elegans), was described and the term RNAi was introduced (Fire et al., 1998). Relevant original studies written in English were retrieved through the search interfaces of WoS using specified search terms (key words) organised in strings. For the selection of the search terms, the following factors were taken into account:

- The key elements of the review questions;

- The terms and indexers used by the authors to describe their documents in the database records;

- The search tools and options given by the electronic databases such as search operators (e.g., AND, OR, NEAR) and filters;

- The number of hits received when using the search terms;

- Major groups of irrelevant studies among the hits;

- Language: only English search terms and taxonomic terms were used.

In the choice of the key elements, their number and syntaxes, a stepwise approach was taken. After the inclusion of each element, a search was performed in WoS to assess the influence of each element and their combinations to the number of hits. The list of studies relevant to the review (presented in Appendix A) was used to define common keywords and assess the relevance of the results in the pilot searches in the database. procedure. The present document is published complying with the transparency principle to which the Authority is subject. It may not be considered as an output adopted by the Authority. The European Food Safety Authority reserves its rights, view and position as regards the issues addressed and the conclusions reached in the present document, without prejudice to the rights of the authors. 
When included in the search string, the terms were modified according to the differences of spelling and other types of variation in the representation (such as the use singular and plural nouns) using asterisks $(*)$ to represent any number of characters that may be attached before or after the term. Quotes (") were used to search exact phrases. The terms which are often used in studies (e.g., NTOs, invertebrates, arthropods, insects, diet, feeding, delivery, ingestion, inoculation) produced large numbers of irrelevant studies when used alone. In order to reduce the number of irrelevant hits, additional conditions were used in the search string, requiring these terms to be positioned within a specified number of words with RNAi terms. This was achieved by using the "NEAR" search operator.

\subsubsection{Search string element 1: Population terms}

The population terms are the primary element of the searches when conducting systematic reviews (EFSA, 2010). The selected population terms describe different types of organisms in the focus of the review. In this review, these population terms include general terms such as: NTOs, invertebrates, model, insects and arthropods; specific population terms include common names and names of major taxonomic groups:

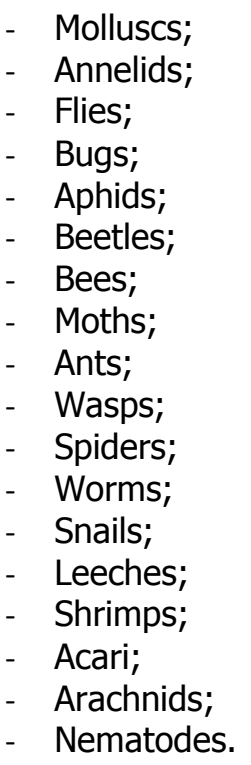

Six major orders of insects containing a large number of target and non-target species (i.e., Coleoptera, Collembola, Diptera, Hemiptera, Hymenoptera and Lepidoptera) were also included among the population terms, as well as terms referring to the developmental stage (i.e., adult, larva, imago, pupae and nymph;) and specific species names (i.e., Drosophila melanogaster and C. elegans). The species names of the well-known model invertebrates $D$. melanogaster and C. elegans were included in the search terms since in some cases the authors do not use other definitions for these organisms (e.g., invertebrate, insect, fly or nematode) in the title, the abstract or the keywords of their studies. The need for inclusion of these terms in the search string was noted during the test of the search string when some of the reference studies used for sensitivity analysis were missing from the results due to the lack of the population term (e.g., Clemens et al. 2000; Marques et al. 2013, see list with reference studies in Appendix A).

The inclusion of $D$. melanogaster and $C$. elegans as terms in the search string resulted in a substantial increase in hits when tested in the WoS. The reason for this was found to be not just because of the large number of studies containing these terms in their title and/or abstract, but also due to the presence of these terms in the "Keywords Plus". The "Keywords Plus" is a special feature of the WoS, and adds additional keywords to the ones provided by the authors that are searchable and automatically assigned by the WoS system based on the references used in the study. While this

The present document has been produced and adopted by the bodies identified above as authors. This task has been carried out exclusively by the author(s) in the context of a contract between the European Food Safety Authority and the authors, awarded following a tender procedure. The present document is published complying with the transparency principle to which the Authority is subject. It may not be considered as an output adopted by the Authority. The European Food Safety Authority reserves its rights, view and position as regards the issues addressed and the conclusions reached in the present document, without prejudice to the rights of the authors. 
might be considered as a useful feature that increases the sensitivity of the search, it produced a large number of irrelevant hits. The analysis of the hits and the major groups of irrelevant studies revealed this effect when using Drosophila and C. elegans and general population terms such as NTOs, adult, arthropod, etc. To limit this effect, the "NEAR" search operator was used to find records where the terms joined by the operator are in the same field (e.g., title or abstract), and within a specified number of words of each other. In this way, the hits only contained studies which included the specified terms in combination (e.g., 20 words distance) with the most common intervention terms RNAi and RNA interference in their title and/or abstract. This also enabled exclusion of studies that have these terms only in the "Keywords Plus". The sensitivity of the search string was confirmed using the 131 reference studies.

When the species names of the model organisms were included among the population terms, the missing studies were present within the search results. These two model organisms are the most commonly used invertebrates in studies that include RNAi, as evident from the preliminary analysis of the search string results.

The following string of population terms was defined:

coleopter* ${ }^{1}$ or collembol*1 or dipter*1 $^{* 1}$ or hemipter*1 or hymenopter*1 $^{* 1}$ or lepidopter*1 ${ }^{*}$ R mollus*1 OR annelid*1 OR acari*1 OR arachn*1 ${ }^{*}$ OR nematod*1 ${ }^{1}$ OR fly ${ }^{1}$ OR flies ${ }^{1}$ OR bug ${ }^{1}$ OR bugs ${ }^{1}$ OR aphid*1 OR beetle*1 OR butterfl*1 OR Apis ${ }^{1}$ OR bee ${ }^{1}$ OR moths ${ }^{1}$ OR moth ${ }^{1}$ OR "ant" ${ }^{1}$ OR "ants" ${ }^{1}$

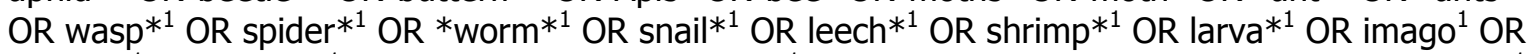
nymph*1 ${ }^{1}$ OR pupa*1 OR "nontarget organism*"1 NEAR/20 RNAi OR "non-target organism*"1 NEAR/20 RNAi OR invertebrate*1 NEAR/20 RNAi OR arthropod* ${ }^{1}$ NEAR/20 RNAi OR insect*1 NEAR/20 *RNA* OR model ${ }^{2}$ NEAR/20 dsRNA OR adult*2 NEAR/20 RNAi OR invertebra*1 NEAR RNAi OR Drosophila ${ }^{2}$ NEAR RNAi OR Drosophila ${ }^{2}$ NEAR dsRNA OR "Caenorhabditis elegans"'2 NEAR RNAi OR "Caenorhabditis elegans" ${ }^{2}$ NEAR dsRNA OR "C. elegans"2 NEAR RNAi OR soil² NEAR *RNA* OR environment*2 NEAR RNA

${ }^{1}$ Keywords included after internal discussion among the review team members;

${ }^{2}$ Keywords included after initial test of the search string and content analysis of the missing reference studies from the retrieved studies.

The NEAR operator was used to limit the number of irrelevant studies and exclude the influence of the "Keywords Plus" terms to the search results in WoS. This enabled combining the keyword with the most common RNAi related terms appearing within a specified number of words from each other.

\subsubsection{Search string element 2: Intervention terms}

The intervention terms include the various types and wordings of RNAi and RNAi molecules such as RNA interference, dsRNA, miRNA and siRNA. These terms limit the query to studies that include RNAi molecules.

The following string of intervention terms was defined:

"double stranded ribonucleic acid*" OR "double-stranded RNA" OR "double stranded RNA" OR RNAi OR "RNA interference" OR "RNA-interference" OR dsRNA OR miRNA OR siRNA OR microRNA OR "small RNA"

In the definition of these terms, the team took into account the various RNA molecules that can be used for RNAi. All the terms were defined after internal discussion and minor adjustments were made during the test of the search string mainly due to the different ways of naming and spelling.

\subsubsection{Search string element 3: Exposure and outcome terms}

As the third element, terms that describe different types of exposure to RNAi molecules and relevant outcomes were defined. This includes various types of exposure and administration terms such as

The present document has been produced and adopted by the bodies identified above as authors. This task has been carried out exclusively by the author(s) in the context of a contract between the European Food Safety Authority and the authors, awarded following a tender procedure. The present document is published complying with the transparency principle to which the Authority is subject. It may not be considered as an output adopted by the Authority. The European Food Safety Authority reserves its rights, view and position as regards the issues addressed and the conclusions reached in the present document, without prejudice to the rights of the authors. 
oral, topical, injection, spray, as well as relevant outcomes such as silencing, knock-out, gene inhibition, gene function, disruption. The inclusion of outcome terms was found necessary since some of the studies do not mention the routes of exposure to RNA molecules in their abstracts.

Most of the exposure terms are common words that are often used in various types of scientific studies. To limit the irrelevant results, the "NEAR" operator was used to make sure that the exposure term is positioned within 15 words distance from any type of RNA molecule in the title or the abstract of the study. For the outcome terms, which consist of two words in combination, the NEAR operator was used to capture differences in wording, e.g., gene NEAR/3 silenc* which covers many forms of wording such as "gene was silenced", "gene is silenced", "gene silencing", "silencing of genes", "silenced gene", "silencing of (name of a gene) gene".

The following string of exposure/outcome terms was defined:

plant* NEAR *RNA OR diet* NEAR *RNA OR deliver* NEAR *RNA OR ingest* NEAR *RNA OR *inject* NEAR *RNA OR inoculat* NEAR *RNA OR topical* NEAR *RNA OR target NEAR/5 gene* OR spray* NEAR *RNA OR oral* NEAR *RNA OR feed* NEAR *RNA OR fed NEAR *RNA OR field OR gene NEAR/3 silenc* OR RNA* NEAR/3 silenc* OR gene NEAR/3 knockdown OR gene NEAR/3 knock-out OR gene NEAR/3 express* OR *RNA NEAR/3 pathway OR *RNA NEAR/3 uptake OR environment* NEAR/3 assessment OR inhibit* NEAR/3 expression OR inhibit* NEAR/3 function* OR disrupt* OR formation NEAR RNAi

\subsubsection{Additional search string elements in order to reduce the number of irrelevant studies in Web of Science}

When the search string containing the three key elements alone was used in WoS, the number of hits was above 30,000 . This number was too large for a manual selection for relevance, and imposed the need to reduce the number of hits without losing sensitivity of the search string. This was achieved by the inclusion of two additional elements in the search string and through the use of the advanced search options of WoS.

Additional element 1: excluding studies in rodents, mammals and humans by title, abstract and key words

The aim of this element of the search string was to exclude studies which involve RNAi with organisms that are not relevant to the review, such as rodents, mammals and humans. This includes terms often used in these types of studies, such as rats, rodents, clinical, patients. The Boolean operator "NOT" was used to exclude the studies that contain the terms in their title, abstract or key words. These terms were defined after carrying out a content analysis of the titles and abstracts of the major groups of irrelevant studies. The lack of these terms in the titles and the abstract of the reference studies was confirmed using text editing program.

The following string of terms that exclude studies in rodents, mammals and humans was defined:

drug* OR rat OR rats OR rodent* OR clinical OR "Stem Cells" OR carcinoma OR leukaemia OR tumor* OR pig OR pigs OR cattle* OR patient*

Additional element 2: excluding studies in rodents, mammals and humans by title.

Major terms that occasionally appear in the abstracts in the relevant studies, but that are never present in the title are used to limit the search results.

The following string of terms that exclude studies in rodents, mammals and humans by the title was defined:

mammal* OR mouse OR human* OR cancer*

The present document has been produced and adopted by the bodies identified above as authors. This task has been carried out exclusively by the author(s) in the context of a contract between the European Food Safety Authority and the authors, awarded following a tender procedure. The present document is published complying with the transparency principle to which the Authority is subject. It may not be considered as an output adopted by the Authority. The European Food Safety Authority reserves its rights, view and position as regards the issues addressed and the conclusions reached in the present document, without prejudice to the rights of the authors. 
In conclusion, the constructed search string consisted of five parts (linked with "AND" or "NOT"), where the terms in each part were linked with "OR". This provided the following full search string that was used in WoS:

TS=(coleopter* or collembol* or dipter* or hemipter* or hymenopter* or lepidopter* OR mollus* OR annelid* OR acari* OR arachn* OR nematod* OR fly OR flies OR bug OR bugs OR aphid* OR beetle* OR butterfl* OR Apis OR bee OR moths OR moth OR "ant" OR "ants" OR wasp* OR spider* OR *worm* OR snail* OR leech* OR shrimp* OR larva* OR imago OR nymph* OR pupa* OR "nontarget organism*" NEAR/20 RNAi OR "non-target organism*" NEAR/20 RNAi OR invertebrate* NEAR/20 RNAi OR arthropod* NEAR/20 RNAi OR insect* NEAR/20 *RNA* OR model NEAR/20 dsRNA OR adult* NEAR/20 RNAi OR invertebra* NEAR RNAi OR Drosophila NEAR RNAi OR Drosophila NEAR dsRNA OR "Caenorhabditis elegans" NEAR RNAi OR "Caenorhabditis elegans" NEAR dsRNA OR "C. elegans" NEAR RNAi OR soil NEAR *RNA* OR environment* NEAR RNA) AND TS=("double stranded ribonucleic acid*" OR "double-stranded RNA" OR "double stranded RNA" OR RNAi OR "RNA interference" OR "RNA-interference" OR dsRNA OR miRNA OR siRNA OR microRNA OR "small RNA") AND TS=(plant* NEAR *RNA OR diet* NEAR *RNA OR deliver* NEAR *RNA OR ingest* NEAR *RNA OR *inject* NEAR *RNA OR inoculat* NEAR *RNA OR topical* NEAR *RNA OR target NEAR/5 gene* OR spray* NEAR *RNA OR oral* NEAR *RNA OR feed* NEAR *RNA OR fed NEAR *RNA OR field OR gene NEAR/3 silenc* OR RNA* NEAR/3 silenc* OR gene NEAR/3 knockdown OR gene NEAR/3 knock-out OR gene NEAR/3 express* OR *RNA NEAR/3 pathway OR *RNA NEAR/3 uptake OR environment* NEAR/3 assessment OR inhibit* NEAR/3 expression OR inhibit* NEAR/3 function* OR disrupt* OR formation NEAR RNAi) NOT TS=(drug* OR rat OR rats OR rodent* OR clinical OR "Stem Cells" OR carcinoma OR leukaemia OR tumor* OR pig OR pigs OR cattle* OR patient*) NOT TI=(mammal* OR mouse OR human* OR cancer*)

\subsubsection{Testing the search strategy}

The search strategy was tested to determine its suitability to retrieve studies relevant to the review topic. The aim of this exercise was to develop the exact content of the search string to be used in the systematic review and was conducted in one of the largest literature databases: WoS.

To test the relevance of the results from the search string in WoS, we used 131 studies (titles are provided in Appendix A), which comply with the inclusion criteria. The compilation was made based on the reference list of six recent reviews on relevant topics and methodological articles, or studies provided by the team members based on their experience in the subject (see Section 2.2.3.2). The presence of the reference studies among the results from the search string was confirmed manually.

When using the defined search string, on 23 July 2016, the number of hits retrieved in WoS was 22,824 (Table 2). However, this number was not the final number of hits since the WoS system did not remove the duplications automatically, but only when the user browsed through all the results. After the duplications were removed, there were 11,643 hits. The relevance check revealed that all the 131 reference studies were among the results.

The present document has been produced and adopted by the bodies identified above as authors. This task has been carried out exclusively by the author(s) in the context of a contract between the European Food Safety Authority and the authors, awarded following a tender procedure. The present document is published complying with the transparency principle to which the Authority is subject. It may not be considered as an output adopted by the Authority. The European Food Safety Authority reserves its rights, view and position as regards the issues addressed and the conclusions reached in the present document, without prejudice to the rights of the authors. 
Table 2: Scoping exercise results (search performed on 23 July 2016)

\begin{tabular}{|c|c|c|c|}
\hline Platform & Search string & $\begin{array}{l}\text { Number } \\
\text { of hits }\end{array}$ & $\begin{array}{l}\text { Presence of } \\
\text { reference } \\
\text { studies }\end{array}$ \\
\hline
\end{tabular}

TS=(coleopter* or collembol* or dipter* or hemipter* or hymenopter* or lepidopter* OR mollus* OR annelid* OR acari* OR arachn* OR nematod* OR fly OR flies OR bug OR bugs OR aphid* OR beetle* OR butterfl* OR Apis OR bee OR moths OR moth OR "ant" OR "ants" OR wasp* OR spider* OR *worm* OR snail* OR leech* OR shrimp* OR larva* OR imago OR nymph* OR pupa* OR "nontarget organism*" NEAR/20 RNAi OR "non-target organism*" NEAR/20 RNAi OR invertebrate* NEAR/20 RNAi OR arthropod* NEAR/20 RNAi OR insect* NEAR/20 *RNA* OR model NEAR/20 dsRNA OR adult* NEAR/20 RNAi OR invertebra* NEAR RNAi OR Drosophila NEAR RNAi OR Drosophila NEAR dsRNA OR "Caenorhabditis elegans" NEAR RNAi OR "Caenorhabditis elegans" NEAR dsRNA OR "C. elegans" NEAR RNAi OR soil NEAR *RNA* OR environment* NEAR RNA) AND TS=("double stranded Web of ribonucleic acid*" OR "double-stranded RNA" OR "double Science stranded RNA" OR RNAi OR "RNA interference" OR "RNAinterference" OR dsRNA OR miRNA OR siRNA OR microRNA

A

$\begin{array}{ll}22824^{a} & 131 \\ 11643^{b} & \text { from } \\ & 131\end{array}$
OR "small RNA") AND TS $=($ plant* NEAR *RNA OR diet*
NEAR *RNA OR deliver* NEAR *RNA OR ingest* NEAR *RNA OR *inject* NEAR *RNA OR inoculat* NEAR *RNA OR topical* NEAR *RNA OR target NEAR/5 gene* OR spray* NEAR *RNA OR oral* NEAR *RNA OR feed* NEAR *RNA OR fed NEAR *RNA OR field OR gene NEAR/3 silenc* OR RNA* NEAR/3 silenc* OR gene NEAR/3 knockdown OR gene NEAR/3 knock-out OR gene NEAR/3 express* OR *RNA NEAR/3 pathway OR *RNA NEAR/3 uptake OR environment* NEAR/3 assessment OR inhibit* NEAR/3 expression OR inhibit* NEAR/3 function* OR disrupt* OR formation NEAR RNAi) NOT TS $=$ (drug* OR rat OR rats OR rodent* OR clinical OR "Stem Cells" OR carcinoma OR leukaemia OR tumor* OR pig OR pigs OR cattle* OR patient*) NOT TI=(mammal* OR mouse OR human* OR cancer*)

(a): Total number of hits.

(b): Number of hits after the removal of duplications.

The presence of all reference studies within the results of the WoS search, and the fact that the search did not produce excessive numbers of hits, indicated the suitability of the search strategy to retrieve relevant studies.

\subsubsection{Search terms and strings used in CAB abstracts}

The search strategy developed for WoS was adapted and transferred in CAB Abstracts. For the reasons explained above, the time span of the search was the same as in WoS, covering from 1998 to July 2016. For the definition of the content and the syntax of the search string the same factors considered for the WoS search were taken into account. The content of the WoS string, all the keywords organised and connected in elements, was transferred to the string for CAB Abstracts. Additionally, the following specifics of CAB Abstracts were considered:

The present document has been produced and adopted by the bodies identified above as authors. This task has been carried out exclusively by the author(s) in the context of a contract between the European Food Safety Authority and the authors, awarded following a tender procedure. The present document is published complying with the transparency principle to which the Authority is subject. It may not be considered as an output adopted by the Authority. The European Food Safety Authority reserves its rights, view and position as regards the issues addressed and the conclusions reached in the present document, without prejudice to the rights of the authors. 
- The search terms should be present in the title, abstract and the keywords defined by the authors;

- The number of hits in CAB Abstracts while testing the string was much smaller than in WoS.

Since there were less searchable fields and less hits in comparison to WoS, and in order to increase the sensitivity of the search, the proximity operator "NEAR" was removed from the string. Thus, the following full search string was used in CAB Abstracts:

(coleopter* or collembol* or dipter* or hemipter* or hymenopter* or lepidopter* OR mollus* OR annelid* OR acari* OR arachn* OR nematod* OR fly OR flies OR bug OR bugs OR aphid* OR beetle* OR butterfl* OR Apis OR bee OR moths OR moth OR ant OR ants OR wasp* OR spider* OR *worm* OR snail* OR leech* OR shrimp* OR larva* OR imago OR nymph* OR pupa* OR "nontarget organism*" OR "non-target organism*" OR invertebrate* OR arthropod* OR insect* OR model OR adult* OR invertebra* OR Drosophila OR "Caenorhabditis elegans" OR "C. elegans" OR soil OR environment*) AND ("double stranded ribonucleic acid*" OR "double-stranded RNA" OR "double stranded RNA" OR RNAi OR "RNA interference" OR "RNA-interference" OR dsRNA OR miRNA OR siRNA OR microRNA OR "small RNA") AND (plant* OR diet* OR deliver* OR ingest* OR *inject* OR inoculat* OR topical* OR "target gene*" OR spray* OR oral* OR feed* OR fed OR field OR "gene silenc*" OR "RNA* silenc*" OR "gene knockdown" OR "gene knock-out" OR "gene express*" OR "*RNA pathway" OR "*RNA uptake" OR "environment* assessment" OR "inhibit* expression" OR "inhibit* function*" OR disrupt* OR formation) NOT (drug* OR rat OR rats OR rodent* OR clinical OR "Stem Cells" OR carcinoma OR leukaemia OR tumor* OR pig OR pigs OR cattle* OR patient*) NOT title:(mammal* OR mouse OR human* OR cancer*) AND yr:[1998 TO 2016])

Complete documentation of the performed searches was made in order to make the process transparent and reproducible. This includes:

- The name of the database;

- The dates of the search for each database and the period searched;

- Any restrictions or filters used (region, language or publication status);

- The full search strategy (all terms and set combinations) and the numbers of records retrieved from each database;

- The total number of records retrieved from the information sources before and after removing duplicates.

All the documented searches and results were recorded and provided as an Endnote file (.enl) (Annex 1).

\subsubsection{Inclusion and exclusion criteria}

In order to be considered relevant for the review questions, and hence to be included in the review, a study needed to comply with each of the following requirements:

- Relevant intervention(s): Any type of exposure (e.g., oral ingestion, injection, spraying, in planta expression, in vitro, in vivo, in laboratory or field conditions, cross-species exchange) to exogenous RNAi molecules (e.g., miRNA, dsRNA or siRNA) or systems;

- Relevant population(s): Any invertebrate species belonging to annelids, nematodes, arthropods and molluscs, or any receiving relevant environment (e.g., soil, above ground, aquatic environments);

- Relevant outcome(s): Knockdown, upregulation and downregulation of gene expression; changes in any enzymatic, metabolic or physiological processes and functions; any survival, developmental, reproduction, behavioural, immunity or longevity effect; any non-target or off target effects and consequences for species concerned; any process related to the fate of RNAi molecules (e.g., uptake, delivery, mobility, transport, stability, persistence, efficacy, functionality, degradation) in invertebrates and the environment.

The present document has been produced and adopted by the bodies identified above as authors. This task has been carried out exclusively by the author(s) in the context of a contract between the European Food Safety Authority and the authors, awarded following a tender procedure. The present document is published complying with the transparency principle to which the Authority is subject. It may not be considered as an output adopted by the Authority. The European Food Safety Authority reserves its rights, view and position as regards the issues addressed and the conclusions reached in the present document, without prejudice to the rights of the authors. 
A study was excluded during the selection process for the following reasons (Reasons for exclusion REx):

- Relevant intervention is missing (REx1);

- Relevant population is missing (REX2);

- Relevant outcome is missing (REx3);

- No original data is reported (this includes records such as patents, books, book chapters and commentary and review publications) (REx4);

- Duplicate data - the same data are reported in another study (REx5); or

- The full text is not accessible and the attempts for acquiring the studies were not successful (REx6).

\subsubsection{Selection of relevant studies}

The selection of relevant studies was done following a two-step process. In the first step, studies complying with the inclusion criteria were selected by two reviewers, initially by title and abstract, and later by screening the full text. The level of agreement between the reviewers was assessed using Kappa statistics (http://www.vassarstats.net/kappa.html).

In the second step, the team separately examined each study selected during the first step, and subsequently indicated which task(s) it related to, and whether it contained original data or it was a review of other studies. The studies were classified as follow:

1. Fully relevant studies - outcomes provide valuable information to one or more of the tasks;

2. Supplemental studies - outcomes do not provide new or substantial evidence useful for any of the review tasks;

3. Studies with unclear relevance - outcomes could not be assigned to any of the review tasks.

\subsubsection{Relevance assessment criteria for studies on environmental exposure (Task 3) and off-target, non-target and unintended effects (Task 5)}

For selecting studies relevant to Task 3 (Section 5.2), components of the environmental exposure routes and factors affecting each of these exposure pathways and fate of dsRNA, siRNA and miRNA were considered for target and NTOs in different environments, specifically referring to the literature concerning RNAi.

Studies from the database obtained with the systematic search described in this section were screened based on the conceptual framework, presented in Figure 1. Three categories of information were deemed relevant for the selection procedures: (1) reports dealing with the molecular characterization of RNA-expressing GM plants; (2) studies investigating possible exposure routes for RNA-expressing GM plants; and (3) reports about the environmental fate of RNA in environmental matrices.

Studies reporting on the production of RNA-expressing GM plants were reviewed by searching in particular for data relative to exogenous RNA expression levels in plant tissues. Studies presenting data on the presence of RNA (or DNA) in environmental matrices and studies presenting information on the possible movement and degradation of RNA (or DNA) in environmental matrices were also considered relevant.

Data were stored in MS Excel (Version 2007 SP3) format and can be easily used for reviewing the outcomes and results and for possibly updating when further studies will become available.

The present document has been produced and adopted by the bodies identified above as authors. This task has been carried out exclusively by the author(s) in the context of a contract between the European Food Safety Authority and the authors, awarded following a tender procedure. The present document is published complying with the transparency principle to which the Authority is subject. It may not be considered as an output adopted by the Authority. The European Food Safety Authority reserves its rights, view and position as regards the issues addressed and the conclusions reached in the present document, without prejudice to the rights of the authors. 


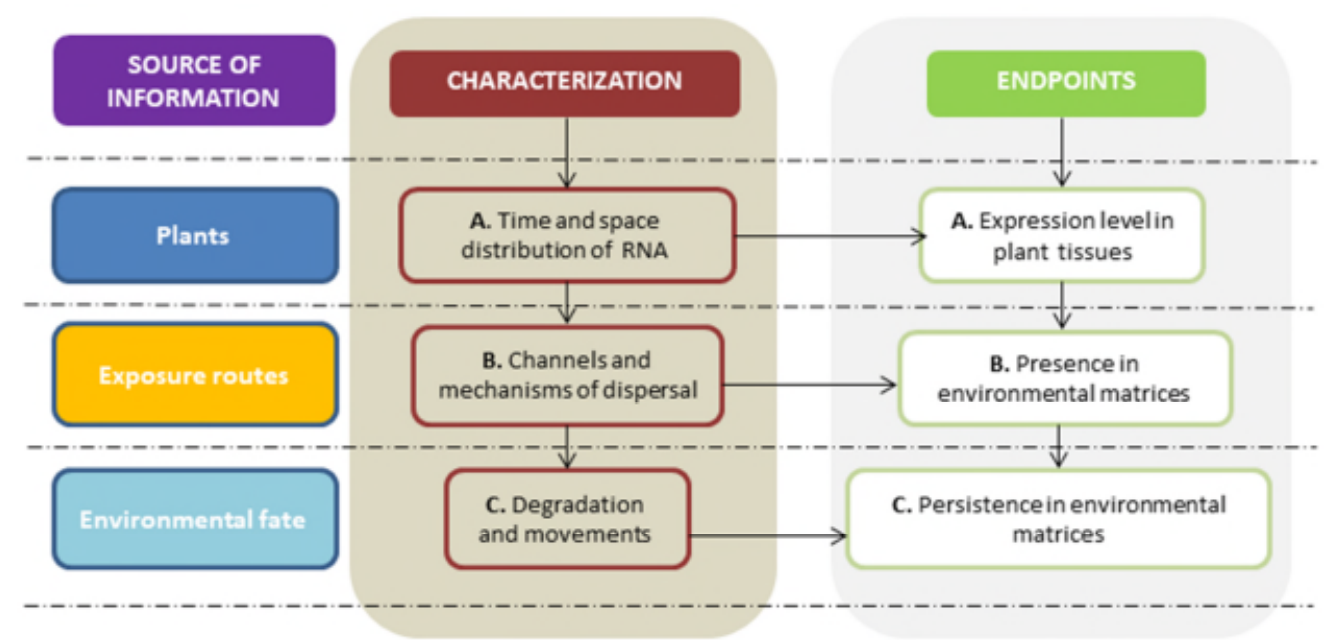

Figure 1: Conceptual framework for selecting studies relevant for the exposure characterization of RNA-expressing GM plants

The list of studies retrieved during the systematic search was screened for relevance to Task 5 (Section 5.4). Articles were deemed relevant if they were dealing with: target specificity of the dsRNA (i.e., bioinformatics analyses and/or gene silencing experiments were conducted comparatively on the target as well as on some non-target species); reports of unintended effects on the target species; bioassays with dsRNA on non-target species; evaluation of trophic chain effects.

In addition, information retrieved from studies published after the systematic search (August 2016 November 2017) were also reviewed.

\subsubsection{Management of the references and documents retrieved}

When possible, the search results were exported from the databases in a format suitable for import in a reference manager program (e.g, "ris" or "ciw"). The EndNote program (EndNote X7) was used to create a library with the overall search results. Duplicate records were removed using the program's function. All the remaining duplications (due to differences in the reference representation) were removed manually. If not possible to be extracted in the reference manager format, the records were saved as plain text or as a table for screening separately from the EndNote library after removal of the duplications.

\subsection{References}

Clemens JC, Worby CA, Simonson-Leff N, et al., 2000. Use of double-stranded RNA interference in Drosophila cell lines to dissect signal transduction pathways. Proceedings of the National Academy of Sciences of the United States of America 97, 6499-6503.

EFSA (European Food Safety Authority), 2010. Application of systematic review methodology to food and feed safety assessments to support decision making. EFSA Journal, 8, 1-90.

EFSA (European Food Safety Authority), 2011. Submission of scientific peer-reviewed open literature for the approval of pesticide active substances under Regulation (EC) No 1107/2009 (OJ L 309, 24.11.2009, p. 1-50). EFSA Journal, 9, 1-9.

Fire A, Xu S, Montgomery MK, Kostas SA, Driver SE and Mello CC, 1998. Potent and specific genetic interference by double-stranded RNA in Caenorhabditis elegans. Nature, 391, 806-811

The present document has been produced and adopted by the bodies identified above as authors. This task has been carried out exclusively by the author(s) in the context of a contract between the European Food Safety Authority and the authors, awarded following a tender procedure. The present document is published complying with the transparency principle to which the Authority is subject. It may not be considered as an output adopted by the Authority. The European Food Safety Authority reserves its rights, view and position as regards the issues addressed and the conclusions reached in the present document, without prejudice to the rights of the authors. 
Kumar A, Wang S, Ou R, Samrakandi M, Beerntsen BT and Sayre, RT, 2013. Development of an RNAi based microalgal larvicide to control mosquitoes. MalariaWorld Journal, 4, No. 6.

Meissle M, Naranjo SE, Kohl C, Riedel J and Romeis, J, 2014. Does the growing of Bt maize change abundance or ecological function of non-target animals compared to the growing of non-GM maize? A systematic review protocol. Environmental Evidence, 3, No. 7.

Nandety RS, Kuo Y-W, Nouri S and Falk BW, 2015. Emerging strategies for RNA interference (RNAi) applications in insects. Bioengineered, 6, 8-19.

Owens $L$ and Malham S, 2015. Review of the RNA interference pathway in molluscs including some possibilities for use in bivalves in aquaculture. Journal of Marine Science and Engineering, 3, 8799.

Roberts AF, Devos Y, Lemgo GN and Zhou, X, 2015. Biosafety research for non-target organism risk assessment of RNAi-based GE plants. Frontiers in Plant Science, 6, Article 958.

Schumpert CA, Dudycha JL and Patel RC, 2015. Development of an efficient RNA interference method by feeding for the microcrustacean Daphnia. BMC Biotechnology, 15, No. 91.

Sweet J and Kostov K, 2014. What are the effects of the cultivation of GM herbicide tolerant crops on botanical diversity? A systematic review protocol. Environmental Evidence, 3, 8.

Zotti MJ and Smagghe G, 2015. RNAi technology for insect management and protection of beneficial insects from diseases: lessons, challenges and risk assessments. Neotropical Entomology, 44, 197-213.

The present document has been produced and adopted by the bodies identified above as authors. This task has been carried out exclusively by the author(s) in the context of a contract between the European Food Safety Authority and the authors, awarded following a tender procedure. The present document is published complying with the transparency principle to which the Authority is subject. It may not be considered as an output adopted by the Authority. The European Food Safety Authority reserves its rights, view and position as regards the issues addressed and the conclusions reached in the present document, without prejudice to the rights of the authors. 


\section{Results of the systematic literature search (Task 1.2)}

\subsection{Search results in Web of Science}

The search in WoS was performed on 24 July 2016 and included all the databases in the subscription of ABI (Table 3). The search used the developed and tested for relevance search string (Table 2), with the time-span of the search from 1998 to July 2016. The search resulted in a total of 11,643 hits. All references were exported as "ciw" files into an EndNote library. The automatic function of the EndNote program "Find Duplicates" was able to find 199 duplications. From the overall results there were 354 references to books and book sections and 272 to patents, which were removed from the list. Thus 10,818 references from the WoS search were left to be screened for relevance.

\subsection{Search results in CAB abstracts}

The search in CAB abstracts was performed on 12 August 2016 and details are summarised in Table 3.

A total of 6,037 hits were retrieved from CAB abstracts. All the results were exported as "ris" files and imported into the EndNote library.

An EndNote library was made uniting the results from the WoS and CAB abstracts searches. It contained all the 17,680 records $(11,643$ from WoS plus 6,037 from CAB abstracts). The automatic "Find Duplicates" function of EndNote was able to detect 1,645 records in CAB abstracts which duplicated records present in the WoS results. Together with the 199 duplicates detected within the WoS results, the total number of duplicates we detected automatically was 1,844 . They were removed from the list, but kept in the "Trash" folder of the library file.

The remaining 10,818 records from WoS and 4,392 records from CAB abstracts were extracted in table format separately from the library and the files were used for an additional, semi-manual comparison of the references in order to identify duplications which could not be detected by the program due to the differences in spelling. This was done by using text comparing plug in MS Excel. In this way another 1,334 CAB abstracts records results duplicating WoS records were found. Finally, the total number of duplicates removed from the CAB abstracts records list was 2,979 $(1,645$ automatically detected, plus 1,334 semi-manually detected) or $49 \%$, leaving 3,058 unique records for screening. Together with the 199 duplicates detected among in the WoS results, the final number of duplicates removed from the list was $3,178 \%$ or $18 \%$ of all the hits.

From the overall 17,680 records, the total number of records retrieved after removing duplicates $(3,178)$, books (82), books sections (272) and patents (272) was $13,876(10,818$ records from WoS, plus 3,058 from CAB abstracts).

\subsection{Selection of relevant studies}

All the records from the EndNote library created as a result of the search were extracted in a table format which was used for the selection of relevant studies.

The initial selection was performed by two reviewers at ABI independently. They assessed whether or not a study fulfiled the inclusion criteria, marking each of the reviewed studies in the MS Excel sheet with YES or NO. The screening was based on the content of the abstracts of the studies, however during the process it was noted that in many cases the amount of information was not sufficient to make a definitive decision. In these cases, the full text of the studies was examined as well.

The present document has been produced and adopted by the bodies identified above as authors. This task has been carried out exclusively by the author(s) in the context of a contract between the European Food Safety Authority and the authors, awarded following a tender procedure. The present document is published complying with the transparency principle to which the Authority is subject. It may not be considered as an output adopted by the Authority. The European Food Safety Authority reserves its rights, view and position as regards the issues addressed and the conclusions reached in the present document, without prejudice to the rights of the authors. 
Table 3: Details of the searches in Web of Science and CAB Abstracts

\begin{tabular}{|c|c|c|}
\hline $\begin{array}{l}\text { Data requirement(s) } \\
\text { captured in the search }\end{array}$ & \multicolumn{2}{|c|}{ Details of the searches } \\
\hline $\begin{array}{l}\text { Intervention(s): Any } \\
\text { type of exposure } \\
\text { (e.g., oral ingestion, } \\
\text { injection, spraying, in } \\
\text { planta expression, in } \\
\text { vitro, in vivo, in laboratory } \\
\text { or field conditions, cross- } \\
\text { species exchange) to } \\
\text { exogenous RNAi } \\
\text { molecules (e.g., miRNA, } \\
\text { dsRNA or siRNA) or } \\
\text { systems. }\end{array}$ & $\begin{array}{l}\text { Database 1: Web of Science } \\
\text { including the following databases: } \\
\text { - Web of Science }{ }^{T M} \text { Core Collection } \\
\text { - BIOSIS Citation Index }{ }^{S M} \\
\text { - Current Contents Connect } \\
\text { - Data Citation Index }{ }^{S M} \\
\text { - Derwent Innovations Index }{ }^{S M} \\
\text { - KCI-Korean Journal Database } \\
\text { - MEDLINE }{ }^{\circledR} \\
\text { - Russian Science Citation Index } \\
\text { - SciELO Citation Index } \\
\text { - Zoological Record }{ }^{\circledR}\end{array}$ & $\begin{array}{l}\text { Database 2: CAB Abstracts (1984- } \\
\text { 2016) (CABI, Wallingford, UK) }\end{array}$ \\
\hline \multirow{2}{*}{$\begin{array}{l}\text { Population(s): Any } \\
\text { invertebrate species } \\
\text { belonging to annelids, } \\
\text { nematodes, arthropods } \\
\text { and molluscs or any } \\
\text { relevant receiving } \\
\text { environment (e.g., soil, } \\
\text { above ground, aquatic) }\end{array}$} & $\begin{array}{l}\text { Justification for choosing the } \\
\text { source: WoS is a platform which } \\
\text { allows simultaneous search across } \\
\text { the major multi-disciplinary } \\
\text { databases for scholarly literature. }\end{array}$ & $\begin{array}{l}\text { Justification for choosing the } \\
\text { source: CABI is a comprehensive } \\
\text { database for the applied life sciences - } \\
\text { agriculture, environment, veterinary } \\
\text { sciences, applied economics, food } \\
\text { science and nutrition. }\end{array}$ \\
\hline & $\begin{array}{l}\text { Date of the search: } 24 \text { July } 2016 \\
\text { Language limitation: no limitation }\end{array}$ & $\begin{array}{l}\text { Date of the search: } 12 \text { August } 2016 \\
\text { Language limitation: no limitation }\end{array}$ \\
\hline \multirow{3}{*}{$\begin{array}{l}\text { Outcome(s): } \\
\text { Knockdown, upregulation } \\
\text { and downregulation of } \\
\text { gene expression; changes } \\
\text { in any enzymatic, } \\
\text { metabolic or physiological } \\
\text { processes and functions; } \\
\text { any survival, } \\
\text { developmental, } \\
\text { reproduction, behavioural, } \\
\text { immunity or longevity } \\
\text { effect; any non-target or } \\
\text { off target effects and } \\
\text { consequences for species } \\
\text { concerned; any process } \\
\text { related to the fate of RNAi } \\
\text { molecules (e.g., uptake, } \\
\text { delivery, mobility, } \\
\text { transport, stability, } \\
\text { persistence, efficacy, } \\
\text { functionality, degradation) } \\
\text { in invertebrates and } \\
\text { the environment. }\end{array}$} & $\begin{array}{l}\text { Date span of the search: } 1998 \text { to } \\
24 \text { July } 2016\end{array}$ & $\begin{array}{l}\text { Date span of the search: } 1998 \text { to } \\
12 \text { August } 2016\end{array}$ \\
\hline & $\begin{array}{l}\text { Search strategies used for this } \\
\text { data requirement }\end{array}$ & $\begin{array}{l}\text { ategies used for this data } \\
\text { nt }\end{array}$ \\
\hline & $\begin{array}{l}\text { TS=(coleopter* or collembol* or } \\
\text { dipter* or hemipter* or } \\
\text { hymenopter* or lepidopter* OR } \\
\text { mollus* OR annelid* OR acari* OR } \\
\text { arachn* OR nematod* OR fly OR } \\
\text { flies OR bug OR bugs OR aphid* OR } \\
\text { beetle* OR butterfl* OR Apis OR } \\
\text { bee OR moths OR moth OR "ant" } \\
\text { OR "ants" OR wasp* OR spider* OR } \\
\text { *worm* OR snail* OR leech* OR } \\
\text { shrimp* OR larva* OR imago OR } \\
\text { nymph* OR pupa* OR "nontarget } \\
\text { organism*" NEAR/20 RNAi OR "non- } \\
\text { target organism*" NEAR/20 RNAi OR } \\
\text { invertebrate* NEAR/20 RNAi OR } \\
\text { arthropod* NEAR/20 RNAi OR } \\
\text { insect* NEAR/20 *RNA* OR model } \\
\text { NEAR/20 dsRNA OR adult* NEAR/20 } \\
\text { RNAi OR invertebra* NEAR RNAi OR }\end{array}$ & $\begin{array}{l}\text { (coleopter* or collembol* or dipter* or } \\
\text { hemipter* or hymenopter* or } \\
\text { lepidopter* OR mollus* OR annelid* OR } \\
\text { acari* OR arachn* OR nematod* OR fly } \\
\text { OR flies OR bug OR bugs OR aphid* OR } \\
\text { beetle* OR butterfI* OR Apis OR bee OR } \\
\text { moths OR moth OR ant OR ants OR } \\
\text { wasp* OR spider* OR *worm* OR snail* } \\
\text { OR leech* OR shrimp* OR larva* OR } \\
\text { imago OR nymph* OR pupa* OR } \\
\text { "nontarget organism*" OR "non-target } \\
\text { organism*" OR invertebrate* OR } \\
\text { arthropod* OR insect* OR model OR } \\
\text { adult* OR invertebra* OR Drosophila OR } \\
\text { "Caenorhabditis elegans" OR "C. } \\
\text { elegans" OR soil OR environment*) AND } \\
\text { ("double stranded ribonucleic acid*" OR } \\
\text { "double-stranded RNA" OR "double } \\
\text { stranded RNA" OR RNAi OR "RNA }\end{array}$ \\
\hline
\end{tabular}

The present document has been produced and adopted by the bodies identified above as authors. This task has been carried out exclusively by the author(s) in the context of a contract between the European Food Safety Authority and the authors, awarded following a tender procedure. The present document is published complying with the transparency principle to which the Authority is subject. It may not be considered as an output adopted by the Authority. The European Food Safety Authority reserves its rights, view and position as regards the issues addressed and the conclusions reached in the present document, without prejudice to the rights of the authors. 
Drosophila NEAR RNAi OR

Drosophila NEAR dsRNA OR

"Caenorhabditis elegans" NEAR RNAi

OR "Caenorhabditis elegans" NEAR

dsRNA OR "C. elegans" NEAR RNAi

OR soil NEAR *RNA* OR

environment* NEAR RNA) AND

$\mathrm{TS}=$ ("double stranded ribonucleic acid*" OR "double-stranded RNA" OR "double stranded RNA" OR RNAi OR "RNA interference" OR "RNAinterference" OR dsRNA OR miRNA OR siRNA OR microRNA OR "small RNA") AND TS=(plant* NEAR *RNA OR diet* NEAR *RNA OR deliver* NEAR *RNA OR ingest* NEAR *RNA OR *inject* NEAR *RNA OR inoculat* NEAR *RNA OR topical* NEAR *RNA OR target NEAR/5 gene* OR spray* NEAR *RNA OR oral* NEAR *RNA OR feed* NEAR *RNA OR fed NEAR *RNA OR field OR gene NEAR/3 silenc* OR RNA* NEAR/3 silenc* OR gene NEAR/3 knockdown OR gene NEAR/3 knockout OR gene NEAR/3 express* OR *RNA NEAR/3 pathway OR *RNA NEAR/3 uptake OR environment* NEAR/3 assessment OR inhibit* NEAR/3 expression OR inhibit* NEAR/3 function* OR disrupt* OR formation NEAR RNAi) NOT $\mathrm{TS}=$ (drug* $\mathrm{OR}$ rat $\mathrm{OR}$ rats $\mathrm{OR}$ rodent* OR clinical OR "Stem Cells" OR carcinoma OR leukaemia OR tumor* OR pig OR pigs OR cattle* OR patient*) NOT TI=(mammal* OR mouse OR human* OR cancer*)

Total number of records

received: 11,643

Total number of records retrieved after removing duplicates, books, books sections and patents: 13,876 interference" OR "RNA-interference" OR dsRNA OR miRNA OR siRNA OR microRNA OR "small RNA") AND (plant* OR diet* OR deliver* OR ingest* OR *inject* OR inoculat* OR topical* OR "target gene*" OR spray* OR oral* OR feed* OR fed OR field OR "gene silenc*" OR "RNA* silenc*" OR "gene knockdown" OR "gene knock-out" OR "gene express*" OR "*RNA pathway" OR "*RNA uptake" OR "environment* assessment" OR "inhibit* expression" OR "inhibit* function*" OR disrupt* OR formation) NOT (drug* OR rat OR rats OR rodent* OR clinical OR "Stem Cells" OR carcinoma OR leukaemia OR tumor* OR pig OR pigs OR cattle* OR patient*) NOT title:(mammal* OR mouse OR human* OR cancer*) AND yr:[1998 TO 2016])

\author{
Total number of records received: \\ 6,037
}

$$
13,876
$$

In the beginning of the selection process, after the first 1,000 records were screened the level of agreement between the two reviewers was assessed using kappa statistics. The calculation based on by the author(s) in the context of a contract between the European Food Safety Authority and the authors, awarded following a tender procedure. The present document is published complying with the transparency principle to which the Authority is subject. It may not be considered as an output adopted by the Authority. The European Food Safety Authority reserves its rights, view and position as regards the issues addressed and the conclusions reached in the present document, without prejudice to the rights of the authors. 
the reviewers' decisions resulted in kappa coefficient of 0.7 , and according to the scale given by Landis and Koch (1977) $)^{1}$ the reviewers agreement is "substantial" $(k>0,6)$.

Studies in which the decision on relevance differed between the two reviewers, were subjected to additional reviewing by another two independent reviewers, i.e., Ghent University and JT Environmental Consultants. Based on their judgment the final decision on relevance was made.

The assignment of the selected studies to one or more of the review questions was made during the accomplishment of the respective tasks in the project proposal. Studies were excluded based on the defined inclusion and exclusion criteria defined in Section 2.2.7.

During the initial screening, the reviewers found that 8,801 records did not fulfil the inclusion criteria or were excluded and these studies were excluded from the list (Figure 2). The 5,075 eligible reviewed studies were those that mostly included exposure of different types of invertebrates to RNA molecules and were marked as relevant by the reviewers. While this number is surprisingly high, to a great extent it is due to the large number of RNAi studies involving the two model organisms: free living nematode $C$. elegans and fruit fly $D$. melanogaster. Both organisms have been used for studying gene functions, metabolic pathways, and a variety of physiological functions.

The selection of the studies continued with the retrieval of the full text of the selected studies from the databases. The full text was used for an additional screening to confirm the presence of the inclusion criteria. During this stage, primary data related to the used invertebrate species and the delivery method of the RNAi molecules was extracted from the relevant studies. As a result, 463 references were removed from the selected studies for the following reasons: no full text (e.g., poster references, meeting abstracts) - 139 references; duplications - 137 references; relevant intervention is missing - 63 references; full text is not in English - 56 references; relevant population is missing 41 references; no original data is present (reviews and commentary publications) - 27 references. Thus, 4,612 full text studies were finally selected and primary data was extracted for all of them.

\subsection{Conclusions on literature search}

The literature searches in online databases WoS and CAB Abstracts resulted in a total number of 17,680 records, which were reduced to 13,867 unique studies after removing duplicates, books, book sections and patents. During the initial screening of these 13,867 records/studies, we have identified eight major methods for delivery of interfering RNA molecules in around 300 invertebrate species belonging to annelids, nematodes, arthropods and molluscs. To what extent the selected studies are relevant (fully relevant; supplemental studies or studies with unclear relevance) to the review questions are discussed in the following sections. After manual relevance assessment, a total of 4,612 studies were considered relevant.

${ }^{1}$ Landis R and Koch GG, 1977. The measurement of observer agreement for categorical data. Biometrics, 33, $159-174$.

The present document has been produced and adopted by the bodies identified above as authors. This task has been carried out exclusively by the author(s) in the context of a contract between the European Food Safety Authority and the authors, awarded following a tender procedure. The present document is published complying with the transparency principle to which the Authority is subject. It may not be considered as an output adopted by the Authority. The European Food Safety Authority reserves its rights, view and position as regards the issues addressed and the conclusions reached in the present document, without prejudice to the rights of the authors. 

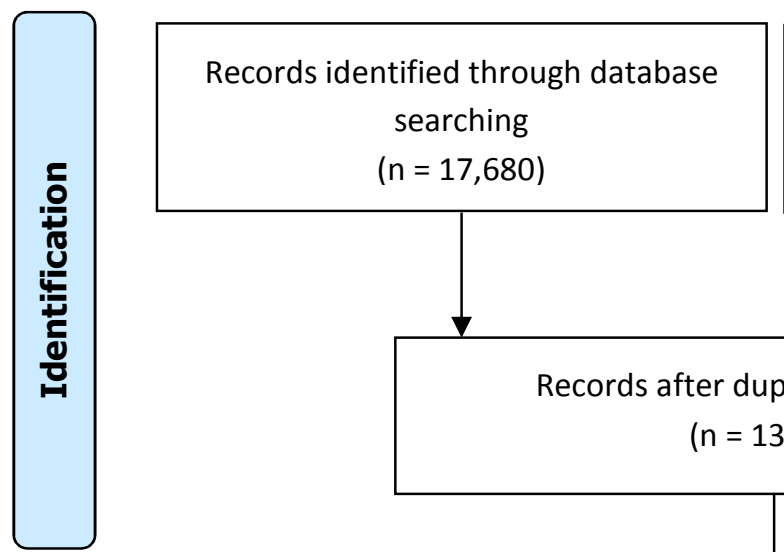

$(n=17,680)$

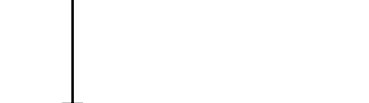

Records after duplicates removed

$(n=13,867)$
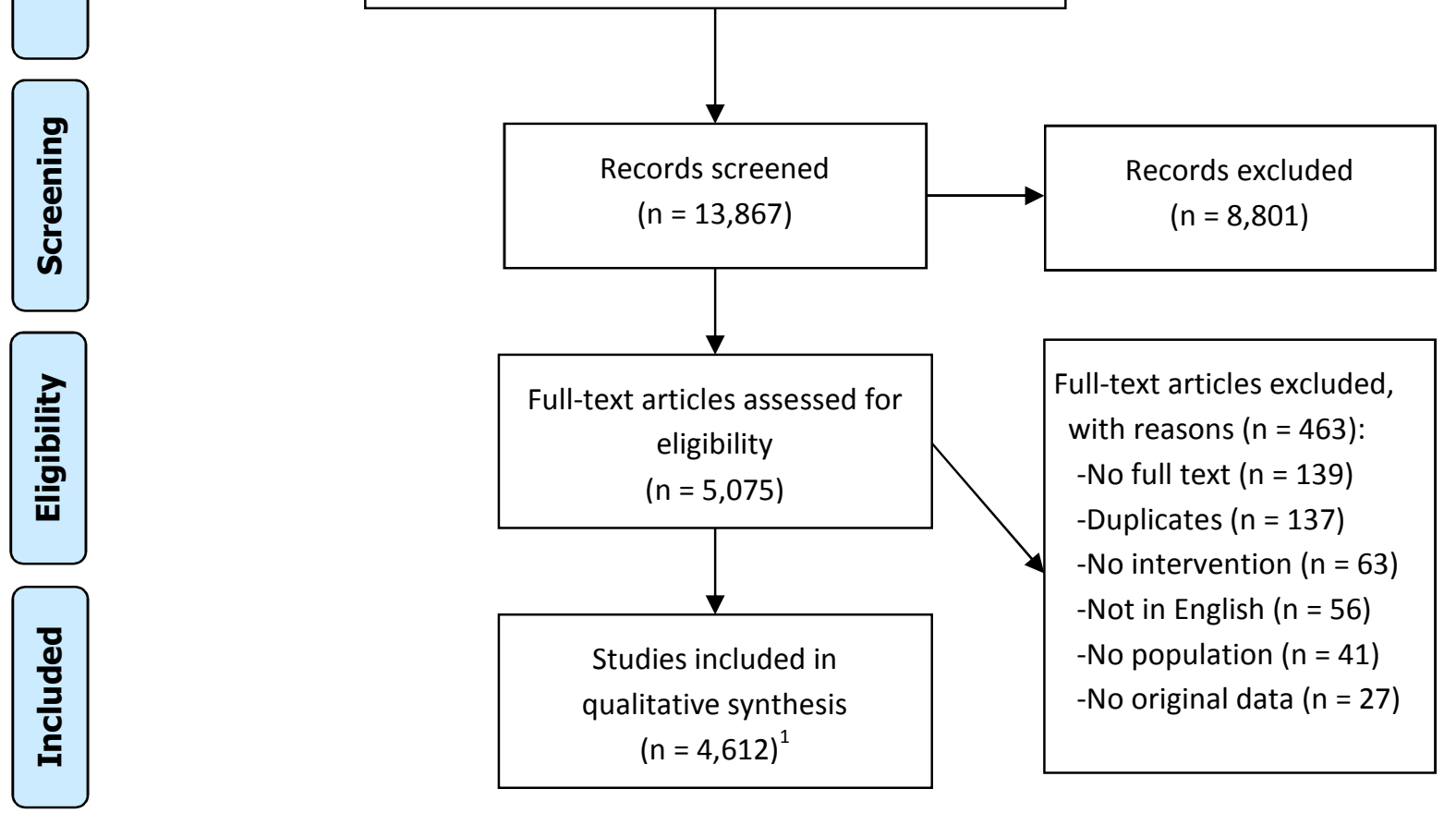

Figure 2: $\quad$ Flow diagram of the selection process
Additional records identified through other sources

$$
(n=0)
$$




\section{Analysis of the primary data from the selected studies}

\subsection{Activity spectrum of the tested dsRNAs, siRNAs and miRNAs in invertebrates (Task 1.3)}

The extracted data was used to create a list of invertebrate species studied in the relevant RNAi publications. The list consists of 341 species (Appendix B) belonging to the four major groups of invertebrates in the focus of this report: annelids, nematodes, arthropods and molluscs. The number of studies in which each species appears is also included in Appendix B.

Arthropods are the most abundant group of species used in the RNAi experiments, representing approximately $62 \%$ of all the studies (Figure 3). The major subgroup is that of the subphylum Hexapoda $(86 \%$, or 2,862 studies), followed by Crustacea $(10 \%$, or 298 studies) and Arachnida (133 studies). Only one study involved the soil-inhabiting springtails (Collembola).

The second largest group of invertebrates are the nematodes with a total of 1,254 studies. The number of RNAi studies which involve molluscs and annelids is much smaller compared to the other two groups of organisms, with 67 and six studies, respectively.

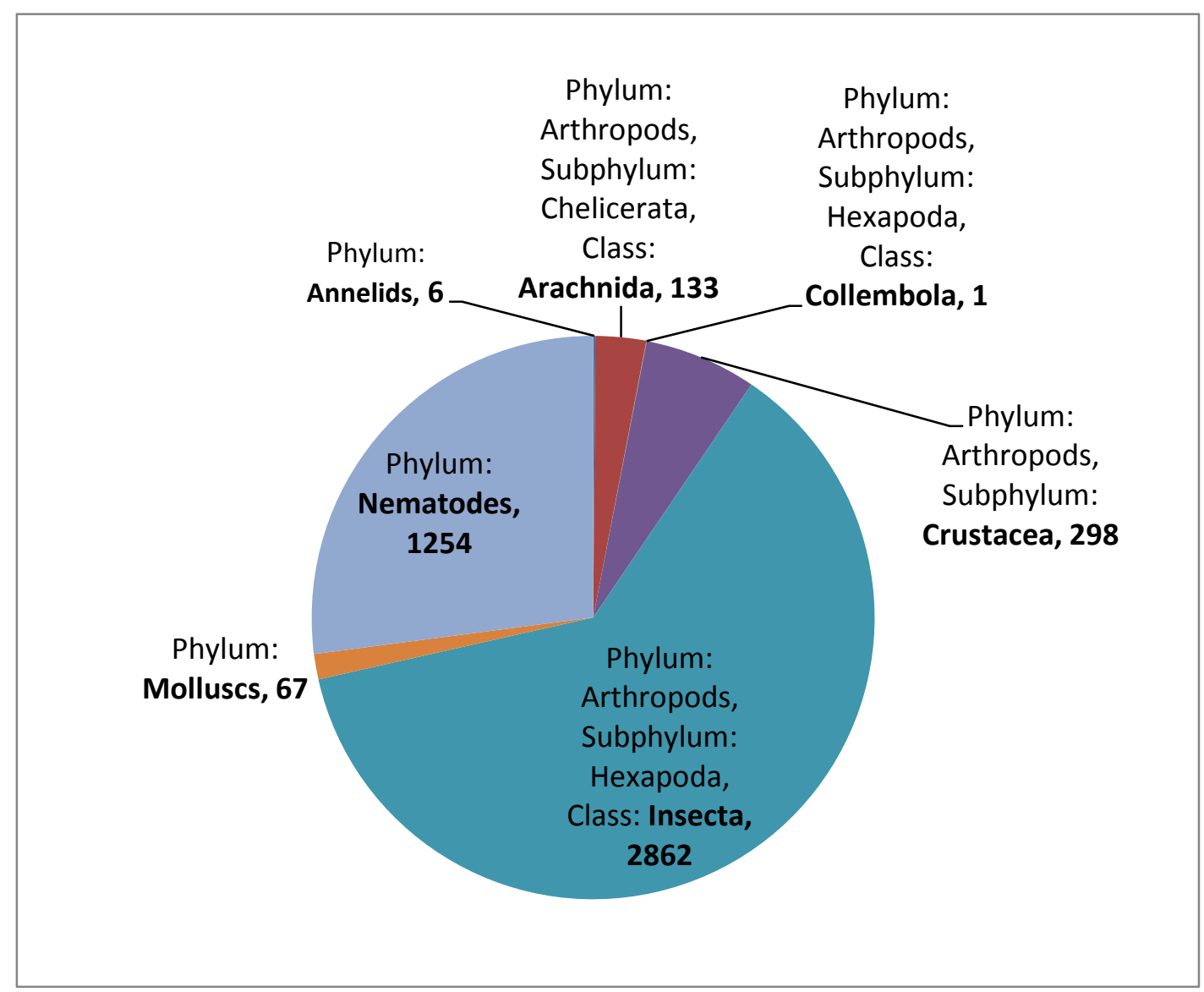

Figure 3: Invertebrates used in RNAi studies retrieved in the systematic literature search. The numbers refer to the number of studies found for each taxonomic group

The present document has been produced and adopted by the bodies identified above as authors. This task has been carried out exclusively by the author(s) in the context of a contract between the European Food Safety Authority and the authors, awarded following a tender procedure. The present document is published complying with the transparency principle to which the Authority is subject. It may not be considered as an output adopted by the Authority. The European Food Safety Authority reserves its rights, view and position as regards the issues addressed and the conclusions reached in the present document, without prejudice to the rights of the authors. 


\subsubsection{Arthropods}

In the conduct of RNAi experiments, the largest group of species from arthropods belong to the hexapods and, more specifically, the insect class. They are represented by more than 180 different species, which is around $60 \%$ of all the studied invertebrate species. Approximately $44 \%$ of all insect RNAi studies involved one species, namely the - the fruit fly $D$. melanogaster (1,243 studies). Following in numbers are the red flour beetle Tribolium castaneum (184 studies) and the silkworm Bombyx mori (176 studies) and, model insects belonging to the orders of Lepidoptera and Coleoptera, respectively (Table 4).

Table 4: $\quad$ Insect species which appear in more than ten RNAi studies

\begin{tabular}{|c|c|c|}
\hline No. & Species & Number of studies \\
\hline 1 & Drosophila melanogaster & 1,243 \\
\hline 2 & Tribolium castaneum & 184 \\
\hline 3 & Bombyx mori & 176 \\
\hline 4 & Aedes aegypti & 133 \\
\hline 5 & Apis mellifera & 78 \\
\hline 6 & Helicoverpa armigera & 67 \\
\hline 7 & Anopheles gambiae & 66 \\
\hline 8 & Nilaparvata lugens & 55 \\
\hline 9 & Plutella xylostella & 42 \\
\hline 10 & Blattella germanica & 39 \\
\hline 11 & Locusta migratoria & 39 \\
\hline 12 & Spodoptera exigua & 36 \\
\hline 13 & Leptinotarsa decemlineata & 32 \\
\hline 14 & Gryllus bimaculatus & 31 \\
\hline 15 & Spodoptera frugiperda & 30 \\
\hline 16 & Manduca sexta & 27 \\
\hline 17 & Rhodnius prolixus & 25 \\
\hline 18 & Spodoptera litura & 25 \\
\hline 19 & Laodelphax striatellus & 24 \\
\hline 20 & Bactrocera dorsalis & 23 \\
\hline 21 & Oncopeltus fasciatus & 22 \\
\hline 22 & Schistocerca gregaria & 21 \\
\hline 23 & Culex pipiens & 19 \\
\hline 24 & Diabrotica virgifera virgifera & 17 \\
\hline 25 & Aedes albopictus & 16 \\
\hline 26 & Glossina morsitans morsitans & 16 \\
\hline 27 & Acyrthosiphon pisum & 15 \\
\hline 28 & Bemisia tabaci & 15 \\
\hline 29 & Nasonia vitripennis & 13 \\
\hline 30 & Myzus persicae & 11 \\
\hline
\end{tabular}

The present document has been produced and adopted by the bodies identified above as authors. This task has been carried out exclusively by the author(s) in the context of a contract between the European Food Safety Authority and the authors, awarded following a tender procedure. The present document is published complying with the transparency principle to which the Authority is subject. It may not be considered as an output adopted by the Authority. The European Food Safety Authority reserves its rights, view and position as regards the issues addressed and the conclusions reached in the present document, without prejudice to the rights of the authors. 
Mosquito species, vectors of human diseases, such as Aedes aegypti (133 studies) and Anopheles gambiae (66 studies) are also frequently studied. The honey bee (Apis mellifera) is also in the top list with 78 studies (Table 4).

The agricultural insect pests most frequently occurring in RNAi studies are four Lepidoptera species (Helicoverpa armigera (67 studies), Plutella xylostella (42 studies), Spodoptera exigua (36 studies), Spodoptera litura (25 studies), the Hemiptera Nilaparvata lugens (55 studies), and the Coleoptera L. decemlineata (32 studies).

Shrimps are the most commonly used crustacean species in RNAi studies, including the ones used as sources of food: Litopenaeus vannamei (88 studies), Penaeus monodon (71 studies), Marsupenaeus japonicus (39 studies) and Fenneropenaeus chinensis (14 studies).

From the class Arachnida, the most commonly occurring species are the ticks Haemaphysalis longicornis (29 studies), Ixodes scapularis (20 studies), Amblyomma americanum (16 studies) and Ixodes ricinus (12 studies).

\subsubsection{Phylum: Nematodes}

The free-living nematode $C$. elegans is involved in 1,109 studies which is nearly $90 \%$ of all the studies within the phylum of nematodes. More than 30 other nematode species are subjects of RNAi experiments, of which the most represented are the plant-parasitic species Meloidogyne incognita (44 studies), Heterodera glycines (16 studies), Bursaphelenchus xylophilus (15 studies), Caenorhabditis briggsae (14 studies), Meloidogyne javanica (nine studies), Heterodera schachtii (eight studies) and Radopholus similis (seven studies).

\subsubsection{Phylum: Molluscs}

The great pond snail (Lymnaea stagnalis - 10 studies), which is a model for neurological studies, and economically important mollusc species, such as the pacific oyster (Crassostrea gigas - 10 studies), pearl oyster (Pinctada fucata - 10 studies) and Farrer's scallop (Chlamys farreri - nine studies), are the most often used species in RNAi studies from this group.

\subsubsection{Phylum: Annelids}

One annelid species, Hirudo medicinalis, is present in six RNAi studies including ring worms. This species has been used as a model organism in cellular analyses of nervous system function.

\subsubsection{Methods used to trigger RNAi in invertebrates}

According to the extracted primary data, the most commonly used method to deliver interfering RNA molecules in invertebrate species is by injection, found in approximately $40 \%$ of the studies. Injecting solutions of RNA molecules in the thorax of adults and larvae at different stages, or directly into eggs or embryos (microinjection), took place in half of the RNAi studies with arthropods. It is also the most used method in molluscs, but less common in nematodes (Table 5).

Oral delivery of dsRNA is the most often used method to trigger RNAi in nematodes. This is partially due to the existence of a routine experimental protocol for RNAi screening of $C$. elegans by feeding worms with dsRNA expressing bacteria. However, the delivery of dsRNA as a supplement to natural or artificial diets or feeding with dsRNA expressing transgenic plants is also being used in many arthropod species.

Incubation and transfection are being used for induction of RNAi in cell cultures and embryos of many arthropods. Soaking, spraying or topical application of dsRNA are other methods for delivery of different groups of invertebrates. In nearly half of the Drosophila studies RNAi was induced by

The present document has been produced and adopted by the bodies identified above as authors. This task has been carried out exclusively by the author(s) in the context of a contract between the European Food Safety Authority and the authors, awarded following a tender procedure. The present document is published complying with the transparency principle to which the Authority is subject. It may not be considered as an output adopted by the Authority. The European Food Safety Authority reserves its rights, view and position as regards the issues addressed and the conclusions reached in the present document, without prejudice to the rights of the authors. 
generating transgenic insects or so-called RNAi lines. We have noted that in 140 studies there is more than one method used for dsRNA delivery.

Table 5: Delivery of interfering RNA molecules in invertebrates

\begin{tabular}{|c|c|c|c|c|c|c|}
\hline Delivery method & Annelids & Arthropods & Molluscs & Nematodes & $\begin{array}{l}\text { Mixed } \\
\text { Phyla }\end{array}$ & Total \\
\hline \multicolumn{7}{|l|}{ Single method studies } \\
\hline $\begin{array}{l}\text { Injection (embryos, eggs, } \\
\text { larvae, adults) }\end{array}$ & 5 & 1,547 & 53 & 208 & 2 & 1,815 \\
\hline Oral & & 314 & & 820 & & 1,134 \\
\hline Transgenic insects & & 743 & & & 1 & 744 \\
\hline $\begin{array}{l}\text { Cells (incubation or } \\
\text { transfection) }\end{array}$ & & 554 & 10 & 10 & & 574 \\
\hline Soaking & 1 & 31 & 2 & 100 & & 134 \\
\hline Transgenic nematodes & & & & 21 & & 21 \\
\hline In silico & & 13 & & 7 & 1 & 21 \\
\hline Other & & 10 & & 1 & 1 & 12 \\
\hline Lysate (cells, embryos) & & 6 & & 2 & & 8 \\
\hline Topical & & 6 & & & & 6 \\
\hline Spraying & & 3 & & & & 3 \\
\hline Multi-method studies & & 57 & 2 & 77 & 4 & 140 \\
\hline Total & 6 & 3,284 & 67 & 1246 & 9 & 4,595 \\
\hline
\end{tabular}

4.2. Oral introduction of SIRNA and miRNA and the induction of efficient RNAi in arthropods, nematodes, annelids and molluscs

For the purpose of ERA baseline information, studies using a feeding or soaking delivery are especially important. Therefore, we have identified all such studies and have prepared an overview table (Annex 2) listing the relevant information for each study, including:

- Species;

- Phylum;

- Order;

- Life stage tested;

- Number of generations investigated;

- RNAi triggering molecule (dsRNA, miRNA, siRNA);

- Length of the RNAi triggering molecule;

- Exposure duration;

- Delivery method (e.g., soaking, feeding bacteria producing dsRNA, in planta, via artificial diet,);

- Target gene;

- The dose or concentration used in the experiment;

- The observed effects of RNAi silencing.

The present document has been produced and adopted by the bodies identified above as authors. This task has been carried out exclusively by the author(s) in the context of a contract between the European Food Safety Authority and the authors, awarded following a tender procedure. The present document is published complying with the transparency principle to which the Authority is subject. It may not be considered as an output adopted by the Authority. The European Food Safety Authority reserves its rights, view and position as regards the issues addressed and the conclusions reached in the present document, without prejudice to the rights of the authors. 


\section{Narrative reviews}

\subsection{Uptake and systemic spread of small RNAs in invertebrates (Task 2)}

For exogenous small RNAs (sRNAs) to have a silencing effect, they need to be internalized into the cell and released into the cytoplasm. Once there, they are then processed further into 20-25 nt long small interfering RNAs (siRNAs) by the RNase III Dicer enzymes, which constitutes the start of the cytoplasmatic RNAi pathway. In this part, we review the current evidence on the cellular uptake mechanisms of sRNAs in invertebrates and the pathways involved in this process. While these mechanisms are still being investigated and under debate, a sizable body of research is already available, mainly in nematodes and arthropods. Here, we explore the similarities and differences between these mechanisms in the different taxonomical groups and we also discuss the implications on the efficiency of RNAi in invertebrate species. Furthermore, we also discuss systemic RNAi, which involves the spread of the silencing signal from the cells or tissues that were exposed to environmental sRNAs to other tissues in the body.

\subsubsection{Introduction}

Whangbo and Hunter (2008) described three concepts relating to RNAi and cellular uptake or transport: (1) cell-autonomous RNAi, which is the RNAi process that happens within a cell after the sRNA has been introduced into the cytoplasm; (2) environmental RNAi, in which the cell is able to take up sRNAs from its environment (e.g., gut lumen, cell medium), leading to silencing in those cells that are exposed to the environmental sRNAs; and (3) systemic RNAi, whereby the sRNAs, after uptake from the environment, are also transported further to neighbouring cells and tissues, eventually leading to RNAi-induced gene silencing there. Systemic RNAi has been shown to be functional in several nematode and arthropod species, including the nematodes $C$. elegans (Hunter et al., 2006; Winston et al., 2002b) and Panagrolaimus superbus (Shannon et al., 2008), the coleopterans T. castaneum (Miller et al., 2012; Tomoyasu et al., 2008a), Di. virgifera (Li et al., 2016) and Aethina tumida (Powell et al., 2017), the honeybee A. mellifera (Aronstein et al., 2006; Jarosch and Moritz, 2011), the cricket Gryllus chico (Dabour et al., 2011), the grasshopper Schistocerca americana (Dong and Friedrich, 2005), the locusts Locusta migratoria (Luo et al., 2013) and Schistocerca gregaria (Wynant et al., 2012), the mollusc C. gigas (Fabioux et al., 2009). However, this is not the case for all invertebrates. In both the nematode and arthropod clades, there are species in which efficient (systemic) RNAi has not been achieved yet, or RNAi success is variable at best. This is the case for example in several lepidopteran, dipteran and hemipteran insect species, as well as animal- and plant-parasitic nematodes and several species from the Caenorhabditis genus (Christiaens and Smagghe, 2014; Christiaens et al., 2014; Terenius et al., 2011). This insensitivity to environmental and systemic RNAi could be linked to several different phenomena, such as a slow or limited cellular uptake, a lack of dsRNA stability in the animal's gut or haemolymph, issues with the cellular core machinery, viral interactions, amplification of the silencing signal, etc. Most of these factors are discussed in Task 4, which deals with the efficiency of RNAi in invertebrates.

In this part, we focus on issues related to cellular uptake of sRNAs, systemic transport and amplification of the silencing signal. Understanding these mechanisms and uptake routes could be vital for assessing possible implications in terms of environmental exposure to sRNAs. We start by discussing the possible entry routes of (dietary) sRNA into the body of invertebrates. Next, an overview is given of what is known so far regarding the cellular uptake of sRNAs and the components that have been identified as being involved in this process. Furthermore, we discuss the evidence available for systemic transport and the mechanisms involved in this process. Finally, the amplification of the silencing signal, which has only really been identified in nematodes, is discussed. Since there is very little knowledge available on these processes in annelids and molluscs, this review mainly focuses on nematodes and arthropods.

The present document has been produced and adopted by the bodies identified above as authors. This task has been carried out exclusively by the author(s) in the context of a contract between the European Food Safety Authority and the authors, awarded following a tender procedure. The present document is published complying with the transparency principle to which the Authority is subject. It may not be considered as an output adopted by the Authority. The European Food Safety Authority reserves its rights, view and position as regards the issues addressed and the conclusions reached in the present document, without prejudice to the rights of the authors. 


\subsubsection{Entry routes of sRNA into the body of invertebrates}

Before going into details on cellular uptake, the first question, when considering exposure to environmental SRNAs, is how these molecules could naturally enter the body of an invertebrate and reach the internal tissues. Quite early on in RNAi research, scientists found that both nematodes and arthropods could be targeted for RNAi by oral ingestion of the sRNAs (Baum et al., 2007b; Soares et al., 2005; Timmons et al., 2001; Timmons and Fire, 1998; Turner et al., 2006; Whyard et al., 2009). Once inside the digestive tract, sRNAs can be taken up intracellularly, by epithelial cells lining the gut, and potentially also passed through to the body cavity by transcytosis, leading to a systemic response (Calixto et al., 2010; Chan and Snow, 2017; Jose and Hunter, 2007; Jose et al., 2009). sRNAs could potentially also pass the digestive tract barrier paracellularly, meaning they are transported by diffusion between the epithelial cells, via the septate junctions. This mode of action was suggested by Chan \& Snow (Chan and Snow, 2017), as this type of transport has been found to occur for a number of other molecules and viruses (Bonning and Chougule, 2014; Casartelli et al., 2007; Fiandra et al., 2009; Hardy et al., 1983; Huang et al., 2015; Jeffers and Roe, 2008). However, to the best of our knowledge, no experimental evidence for paracellular transport of sRNAs in invertebrates is available.

Several studies in both nematodes and arthropods have shown that topical application, by spraying or soaking for example, could also trigger an efficient silencing response (Bakhetia et al., 2005; Fanelli et al., 2005; Gu and Knipple, 2013; Killiny et al., 2014; Pridgeon et al., 2008; Tabara et al., 1998; Wang et al., 2011; Whyard et al., 2009). At present, topical uptake routes have not been investigated thoroughly and the exact uptake mechanisms are not well understood. It is known that, for nematodes, the cuticle is permeable to some degree for water molecules, certain ions and nonelectrolytes including some organic nematicides (Bird and Bird, 2012). Whether this is also the case for nucleic acids is unclear. It has been hypothesized that differences in cuticle permeability could be a factor in explaining the variability in RNAi efficiency observed between different nematode species (Dalzell et al., 2011a). However, we were unable to find direct evidence for transport of sRNAs through the cuticle. An alternative explanation for successful RNAi using soaking as the delivery method could be the fact that the dsRNA is actually taken up orally from the soaking solution. In fact, fluorescence microscopy experiments performed by Urwin et al., (2002) showed that soaking $C$. elegans in a solution containing fluorescein isothiocyanate (FITC) led to oral uptake, rather than uptake through the integument This hypothesis might also explain why the silencing of $s i d-2$ in C. elegans, a gene coding for a protein which is critical for SRNA-uptake in the gut, led to a strong decrease in RNAi efficiency, both in feeding and soaking experiments (Winston et al., 2007).

In insects, the chitin-based cuticle itself is thought to be impenetrable, protecting the insect from desiccation. Therefore, topical uptake of sRNAs has to happen in another way. One plausible theory is that the topically applied SRNAs enter the body and reach the internal tissues through the tracheal system (Gu and Knipple, 2013). The tracheal system is comprised of a network of branched tubes inside the insect, which are connected to the outside environment through valved spiracles on the cuticle of the insects. Through these spiracles, air can enter into the trachea, where gas exchange can happen and oxygen can be taken up. However, we found no direct evidence of tracheal uptake of sRNAs in arthropods. A number of insect dsDNA viruses, for example nuclear polyhedrosis viruses and baculoviruses, have been shown to infect tracheoblasts, but these are considered to be secondary infections coming from the midgut (Barrett et al., 1998; Clem and Passarelli, 2013; Engelhard et al., 1994).

Another possible entry way was proposed by Killiny et al., (2014), who managed to induce RNAi by topically applying a dsRNA solution on the thorax of adult citrus psyllids (Diaphorina citri). They hypothesized that the dsRNA could enter the body through the intersegmental membranes of the thorax. Direct proof for uptake through the integument was provided by Wang et al. (2011), using a topical application of fluorescently labelled dsRNA in eggs and larvae of the Asian corn borer Ostrinia furnacalis.

The present document has been produced and adopted by the bodies identified above as authors. This task has been carried out exclusively by the author(s) in the context of a contract between the European Food Safety Authority and the authors, awarded following a tender procedure. The present document is published complying with the transparency principle to which the Authority is subject. It may not be considered as an output adopted by the Authority. The European Food Safety Authority reserves its rights, view and position as regards the issues addressed and the conclusions reached in the present document, without prejudice to the rights of the authors. 
Whyard et al. (2009) published a study investigating several delivery strategies in insects and found that after soaking one-day old $D$. melanogaster larvae in a dsRNA solution targeting a GUS construct led to a small degree of transcript silencing (5-8\%), but only in gut tissues. Addition of Lipofectamine to the dsRNA solution, a transfection agent which can increase cellular uptake efficiency, increased the silencing efficiency to $50 \%$ in isolated guts. No silencing in other tissues was reported. Adding a colouring dye to the dsRNA solution also confirmed that the liquid was merely ingested, and no evidence for transport through the integument or via the trachea could be found. These findings showed that in soaking experiments, a silencing effect due to oral uptake, rather than uptake through the cuticle cannot be excluded. Given the fact that entry through parts of the body integument has been shown in other insect species, as noted earlier, it also raises the question about variability in uptake routes and uptake mechanisms between different species and different orders within the Insecta.

\subsubsection{Cellular uptake and systemic transport}

Much of what we know about RNAi, including sRNA cellular uptake mechanisms, was first reported in nematodes, more specifically in $C$. elegans. However, given the recent interest in the use of RNAi for pest control, more and more knowledge is being gathered on the molecular processes that drive RNAi in arthropods as well. In this section, we review the information available on cellular uptake and systemic transport in invertebrate species, since both are strongly linked. Given the considerable differences between different invertebrate subphyla in terms of sRNA cellular transport mechanisms and pathways, this part will be structured per subphylum.

\subsubsection{Nematodes}

Since the discovery of RNAi in the free-living nematode C. elegans in 1998 (Fire et al., 1998), this species has become a model organism for RNAi research. Most of the molecular mechanisms involved in RNAi, including those of cellular uptake and systemic spread of sRNAs, were first described in this species. We review what is known on cellular uptake and transport of sRNAs in C. elegans, and discuss these systems in other nematodes, and the differences that exist.

\section{Cellular uptake and transport of sRNAs in Caenorhabditis elegans}

The complete picture of the cellular uptake of SRNAs in C. elegans has not yet been elucidated. However, several different types of proteins and mechanisms have been shown to be involved in this process, including certain elements from the endocytosis pathway and dsRNA-specific receptors or importers (Jose, 2015; Saleh et al., 2006b). Several Systemic RNA Interference Deficient (sid) genes have been discovered and characterized in nematodes and their involvement in uptake, export and systemic spread of sRNAs has been described (Feinberg and Hunter, 2003; Hinas et al., 2012; Jose et al., 2012; Krautz-Peterson et al., 2010; McEwan et al., 2012; Rocheleau, 2012; Shih et al., 2009; Shih and Hunter, 2011; Winston et al., 2002a). Despite the common name, these genes encode for proteins that belong to different protein families and have different working mechanisms and functions, besides all being involved in cellular uptake or transport of the silencing signal. Some of these sid genes are mainly active in the gut tissue, while some are active in other tissues and are involved in systemic transport. Besides the sid genes, several other components involved in cellular uptake have been identified as well, such as RNAi spreading-defective (Rsd) genes and components of the endocytosis pathway. A schematic overview of sRNA uptake pathways in $C$. elegans is given in Figure 4. Additionally, Table 6 lists all genes that have been implicated so far in feeding RNAi and systemic transport in C. elegans. The table is based on the table published by Saleh et al. (2006b) and expanded with additional known elements of the feeding/systemic RNAi pathway.

The present document has been produced and adopted by the bodies identified above as authors. This task has been carried out exclusively by the author(s) in the context of a contract between the European Food Safety Authority and the authors, awarded following a tender procedure. The present document is published complying with the transparency principle to which the Authority is subject. It may not be considered as an output adopted by the Authority. The European Food Safety Authority reserves its rights, view and position as regards the issues addressed and the conclusions reached in the present document, without prejudice to the rights of the authors. 


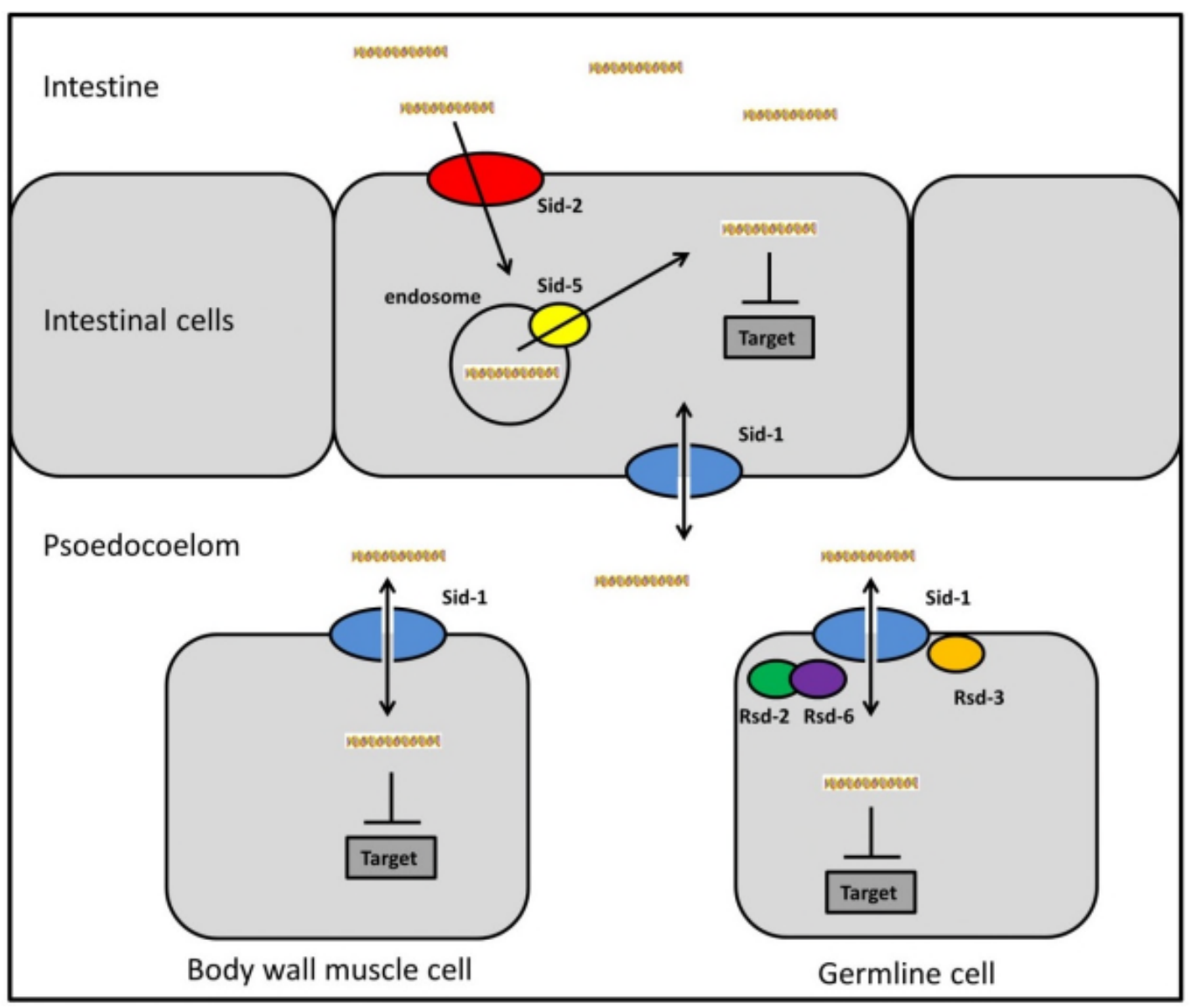

Figure 4: Overview of our current understanding of cellular uptake and transport of dsRNA in Caenorhabditis elegans. A. The situation in cells lining the intestinal lumen involves Sid2 , and has a dual role for Sid-1. Uptake from the intestinal lumen is facilitated by Sid-2. Sid-1 is involved in the release into the cytoplasm. Uptake from the body cavity is facilitated by Sid-1 directly, in association with Sid-3 B. In non-intestinal cells, Sid-2 is not involved and environmental uptake is mainly facilitated by Sid-1 and Sid-3. In both locations, the endosome-associated Sid-5 is involved in export out of the cell and transport of the silencing signal. In gonad cells, Rsd-2, Rsd-3 and Rsd-6 are thought to be involved in cellular uptake, although their exact roles are unknown. More recent research indicates that they might have an indirect effect through the biogenesis of secondary siRNAs (Zhang et al., 2012). Figure based on (Jose and Hunter, 2007); (Dalzell et al., 2011); (Rocheleau, 2012); (McEwan et al., 2012), (Meng et al., 2013) and (Sarkies and Miska, 2014)

Sid-1

Using C. elegans mutant strains which were resistant to systemic RNAi, but not to autonomous RNAi, Sid-1 was first discovered in 2002 as a protein which is required for systemic RNAi. It is expressed in all non-neural cells and possesses predicted transmembrane domains (Winston et al., 2002). The same research group published a study in 2003, where the C. elegans sid-1 gene (CeSid-1) was expressed in Drosophila Schneider 2 (S2) cells, which have a strong cell-autonomous RNAi response, but lack a sid-1 homologue of their own and an environmental RNAi response. Upon expression of CeSid-1, these S2 cells showed a 25- to 130-fold increase of dsRNA uptake, indicating its role in the

The present document has been produced and adopted by the bodies identified above as authors. This task has been carried out exclusively by the author(s) in the context of a contract between the European Food Safety Authority and the authors, awarded following a tender procedure. The present document is published complying with the transparency principle to which the Authority is subject. It may not be considered as an output adopted by the Authority. The European Food Safety Authority reserves its rights, view and position as regards the issues addressed and the conclusions reached in the present document, without prejudice to the rights of the authors. 
cellular uptake of dsRNA. The study also showed that the transport via Sid-1 is passive (Feinberg and Hunter, 2003). Since $C$. elegans sid-2 mutants were found to be resistant to environmental RNAi, it seemed clear that sid-1 alone is not sufficient to fully explain environmental cellular uptake (Winston et al., 2007). McEwan et al. (2012) later showed that, in cells lining the intestinal lumen, it is actually part of a multi-step uptake mechanism involving endocytosis-mediated uptake. First, dsRNA in the intestinal lumen is specifically recognized by Sid-2 membrane proteins on the cell membrane and then taken up by endocytosis. Sid-1 is then responsible for the release of dsRNA from the endosomes into the cytoplasm (McEwan et al., 2012). This also explains the observation that in sid-1 defective intestinal cells, environmental RNAi was not functional, but transport across the intestine was found to occur (Winston et al., 2007).

The involvement of endocytosis in SRNA uptake in C. elegans was unexpected, since earlier studies suggested no involvement in these import (or export) processes (Tijsterman et al., 2004). Further studies also showed that besides dsRNA, Sid-1 is capable of importing pre-miRNA and hairpin-RNA. However, this uptake did not occur as efficiently as 500 bp long dsRNA, possibly due to stretches of single-stranded RNA in those sRNAs (Shih and Hunter, 2011). Finally, a study from José et al. in 2009 also demonstrated that Sid-1 is not necessary for export of sRNAs out of the cell, transport across the cells lining the intestinal lumen or further spreading of the systemic silencing signal (Jose et al., 2009).

Sid-2

The protein encoded by the sid-2 gene is a membrane-bound protein which has been shown to be required for environmental RNAi in C. elegans (McEwan et al., 2012; Winston et al., 2007). Winston et al. (2007) first demonstrated that Sid-2 mutants were resistant to RNAi by bacterial delivery, while transgene-mediated RNAi was successful in these mutants. The study also showed that Sid-2, contrary to Sid-1, was not necessary for export of sRNAs out of the cells and subsequent spread. It is expressed mainly in cells lining the intestinal lumen and was found not to be necessary for uptake of silencing information in muscle cells (Winston et al., 2007). McEwan et al. (2012) reported that the Sid-2 transmembrane receptor is pH-dependant and only active in acidic environments. Furthermore, it was shown to be selective for dsRNA of 50-1,500 bp long. The same study also discovered that the Sid-2 uptake system is linked to vesicle transport and endocytosis, whereby Sid-2 is the receptor selectively binding dsRNA from the intestinal lumen before endocytosis-mediated uptake occurs. McEwan et al. (2012) also suggested that similar systems, using tissue-specific and environmentspecific proteins like Sid-2, could exist in other tissues as well to transport dsRNA to environments which are more suitable for broadly expressed proteins such as Sid-1 to function.

Sid-3

Recently, a tyrosine kinase encoded by the gene sid-3 was discovered and was shown to be implicated in cellular uptake of dsRNA in C. elegans (Jose et al., 2012). This kinase is a homolog of the mammalian activated cdc-42-associated kinase (ACK), which is known to be directly associated with endocytic vesicles (Jose et al., 2012; Teo et al., 2001; Yang et al., 2001). José et al. found the Sid-3 protein to be localized in the cytoplasm of many $C$. elegans tissues and, using sid-3 mutants, found that this protein was required for efficient environmental RNAi, including uptake of dsRNA from the intestine. However, some silencing in the sid-3 mutants was still observed, contrary to what was observed in for example sid-1 mutants. Cell-autonomous RNAi was not affected by the absence of Sid-3, and the researchers could also prove Sid-3 is only involved in import, and not export, of dsRNA (Jose et al., 2012).

\section{Sid-5}

In 2012, the systemic RNAi pathway expanded further with the discovery of Sid-5, a protein encoded by the sid-5 gene and which is found to be associated with the endosomes of somatic cells (Hinas et al., 2012). Hinas et al. discovered that $C$. elegans sid-5 mutants are refractory to systemic RNAi and are only partially sensitive to environmental RNAi (Hinas et al., 2012). While these effects are similar

The present document has been produced and adopted by the bodies identified above as authors. This task has been carried out exclusively by the author(s) in the context of a contract between the European Food Safety Authority and the authors, awarded following a tender procedure. The present document is published complying with the transparency principle to which the Authority is subject. It may not be considered as an output adopted by the Authority. The European Food Safety Authority reserves its rights, view and position as regards the issues addressed and the conclusions reached in the present document, without prejudice to the rights of the authors. 
to those observed in sid-3 mutants, the protein encoded by sid-5 functions entirely differently. Immunohistochemistry experiments revealed that sid-5 is expressed in somatic cells and is associated with late-endosomes in the cells (Hinas et al., 2012). These late endosomes have been implicated as sites of RNAi activity within cells (Gibbings and Voinnet, 2010; Lee et al., 2009). Experiments performed with sid-5 mutants showed that rescue of sid-5 expression in intestinal cells only restored successful RNAi in body wall muscle (bwm) cells, while rescue of sid-5 in bwm cells only, did not recover functional RNAi in these cells, demonstrating that Sid-5 is necessary for transport across the intestine and spreading of the silencing signal. This is contrary to what was discovered for Sid-1, where rescue in the intestine had no effect on bwm cell RNAi functionality, but rescue in bwm cells successfully recovered RNAi there (Hinas et al., 2012; Jose et al., 2009).

\section{Rsd genes}

Similar to the sid genes, RNA Spreading Defective (rsd) genes comprise a group of structurally and functionally diverse proteins. What they have in common is that they are somehow involved in systemic RNAi and the spreading of the silencing signal in C. elegans. Tijsterman et al. (2004) discovered these genes through screens with mutant worms, which were defective for systemic RNAi. They identified 5 genes, which they divided into two groups based on the mutant phenotype; $r s d-4$ (identified as sid-2) and $r s d-8$ (found to be an allele of Sid-1 eventually (Winston et al., 2002)) (Class I) mutations resulted in worms which were defective for RNAi of both somatic and germline specific genes, while $r s d-2, r s d-3$ and $r s d-6$ (Class II) mutations led to worms which were only refractory to RNAi in the germline cells but did not impair initial uptake from the intestine and transport to somatic cells.

The exact role in the cellular uptake or transport mechanisms has not been elucidated yet for most of these genes. One of the best characterized RSD proteins is RSD-3, a homolog of epsinR in mammalian cells, which is implicated in clathrin-mediated vesicular transport (Legendre-Guillemin et al., 2004). Originally thought to be a germline cell-specific gene (Tijsterman et al., 2004), Imae et al. (2016) recently found that the gene is ubiquitously expressed, also in somatic cells, and is required for systemic RNAi in both tissue types. Co-localization studies showed that RSD-3 is associated with the Trans-Golgi-network (TGN) and endosomal vesicles. The rsd-3 mutation caused only a partial resistance to RNAi in somatic cells, contrary to for example knockout of sid-1. The study also showed that RSD-3 is not involved in cellular uptake from, or export out of, intestinal cells, but is required for subsequent uptake from the psoedocoelom. Given these characteristics, the authors hypothesized that RSD-3 might be associated with the same transport pathway involving Sid-1, which is also implicated in uptake and internal cellular transport of sRNAs (Imae et al., 2016).

Tijsterman et al. (2004) further reported that RSD-2 exhibited no known motifs or close homologs in other organisms that could give us a clue about its function, while RSD- 6 contains a Tudor domain, frequently found in RNA-binding proteins. Yeast two-hybrid experiments showed that RSD-2 and RSD-6 potentially act as a complex. Zhang et al. (2012) demonstrated that RSD-2 and RSD-6 are required for the accumulation of secondary siRNAs in $C$. elegans, which could explain these earlier observations, and would imply that they are not directly affecting sRNA uptake. Furthermore, both are also implicated in maintaining chromosome integrity (Han et al., 2008), and in antiviral RNAi through the production of secondary viRNAs (Guo et al., 2013).

\section{fed genes}

Timmons et al. (2003) identified two more mutants, which were insensitive to RNAi by feeding, but did exhibit systemic RNAi when the dsRNA was injected. However, the genes involved in these mutants were not further investigated at that time. fed-1 and fed-2 are likely to be allelic with sid2/rsd-4 and rsd-2, respectively, based on phenotype and genetic map position (Jose and Hunter, 2007; Whangbo and Hunter, 2008).

\section{Other genes or pathways involved in uptake and systemic spread}

The present document has been produced and adopted by the bodies identified above as authors. This task has been carried out exclusively by the author(s) in the context of a contract between the European Food Safety Authority and the authors, awarded following a tender procedure. The present document is published complying with the transparency principle to which the Authority is subject. It may not be considered as an output adopted by the Authority. The European Food Safety Authority reserves its rights, view and position as regards the issues addressed and the conclusions reached in the present document, without prejudice to the rights of the authors. 
At least twelve additional proteins which are likely to be involved in uptake and systemic spread of sRNAs were identified after a genetic screen for feeding RNAi in Drosophila S2 cells was performed by Saleh et al. (2006a). C. elegans homologs of the genes, which were found to be involved in Drosophila feeding RNAi, were knocked out in worms and the effect on feeding RNAi was observed. Most genes which were found in this screen were linked to cellular vesicular transport, such as vacuolar protein sorting-41 (vps-41), conserved oligomeric Golgi complex subunit 2 (cgo-2) and ADPribosylation factor-like protein $1(\mathrm{arl}-1)$. Furthermore, genes involved in lipid metabolism were also identified, together with a few genes of unknown function. These data further proved that endocytosis and vesicular transport is critical in dsRNA uptake and systemic spread. However, the exact role of these genes in the different uptake and transport pathways has not been further investigated yet. A full list of these genes is given in Table 6 .

Table 6: Overview of all genes implicated in Caenorhabditis elegans feeding RNAi uptake and systemic spread (adapted from Saleh et al. (2006b))

\begin{tabular}{lcc}
\hline Group & C. elegans gene ID & C. elegans gene name \\
\hline Sid genes & C04F5.1 & Sid-1 \\
& ZK520.2 & Sid-2 \\
& B0302.1 & Sid-3 \\
Rsd genes & F14B8.2 & Sid-5 \\
& F52G2.2 & Rsd-2 \\
Vesicle mediated transport & C34E11.1 & Rsd-3 \\
Intracellular transport & F16D3.2 & Rsd-6 \\
& F54C9.10 & arl-1 \\
& F22G12.5 & \\
Lipid metabolism & C06G3.10 & cgo-2 \\
Other & ZK1098.5 & trpp-3 \\
Unknown & F32A6.3 & vps-41 \\
& R01H2.5 & ger-1 \\
& B0025.1 & vps-34 \\
& B0464.4 & bre-3 \\
\hline
\end{tabular}

Finally, Sundaram et al. (2006) reported the involvement of at least one ATP binding cassette (ABC) transporter, encoded by haf- 6 , in environmental RNAi of $C$. elegans . In their study, RNAi by injection, soaking and feeding was applied on wild type and haf- 6 mutants. The experiments demonstrated that the haf- 6 mutants had become less sensitive to oral RNAi in germline cells, in a concentrationdependant manner. Interestingly, the assays also showed loss of RNAi phenotype in intestine cells, but not in other somatic cells. Subsequent localization experiments provided an explanation when it was discovered that haf-6 is mainly expressed in intestinal and germline cells and thus the loss of RNAi sensitivity in these haf-6 mutants mainly occurs in the cells where haf- 6 is normally expressed in wild type forms. However, exactly how haf- 6 influences RNAi and whether it is really related to uptake or transport is not known. $A B C$ transporters in general are known to be able to transport substrates in or out of the cells, but also between intracellular compartments, so an involvement in transport of the silencing signal is likely.

The present document has been produced and adopted by the bodies identified above as authors. This task has been carried out exclusively by the author(s) in the context of a contract between the European Food Safety Authority and the authors, awarded following a tender procedure. The present document is published complying with the transparency principle to which the Authority is subject. It may not be considered as an output adopted by the Authority. The European Food Safety Authority reserves its rights, view and position as regards the issues addressed and the conclusions reached in the present document, without prejudice to the rights of the authors. 
A more recent study suggested involvement of another $A B C$ transporter, haf-2, in the RNAi machinery upon exposure to a non-coding RNA (ncRNA) called OxyS from the Escherichia coli bacteria used in the feeding studies (Liu et al., 2012a). This OxyS ncRNA contains a 17nt homologous sequence to the C. elegans gene che-2, encoding a G-protein containing WD40 protein. The authors observed depletion in che-2 mRNA upon feeding on $E$. coli and linked this to a silencing effect induced by OxyS uptake. In their investigation, knockout of sid-1 and sid-2 revealed no change in silencing efficiency, suggesting a different pathway being involved in uptake. Mutants in which haf-2 was knocked out affected the observed che-2 silencing, suggesting an involvement of this ABC transporter (Liu et al., 2012a). However, Akay et al. (2015) further investigated this phenomenon, using OxySoverexpressing and -knockout strains small RNA mapping and came to the conclusion that che-2 silencing was not caused by OxyS ncRNA and that the latter does not cause RNAi in $C$. elegans. In a recent review, Waqas and Shan pointed to the fact that in the small RNA screens performed by Akay et al., there was a shift from 21 nt to $22 \mathrm{nt}$ RNAs between the wild type nematodes and RNAi deficient nematodes used in the study, which was not noticed or mentioned by the authors. Furthermore, the review also points out that different development stages were used in both studies, which can make comparisons difficult (Waqas and Shan, 2016).

\section{Cellular uptake in other nematode species}

In the past 15 years, it has become clear that the mechanisms in C. elegans are not always representative of the whole nematode subphylum. Indeed, several species such as the soil nematodes Caenorhabditis briggsae (Winston et al., 2007), Caenorhabditis remanei (Winston et al., 2007), Caenorhabditis brenneri (Winston et al., 2007), Oscheius tipulae (Louvet-Vallée et al., 2003; Wheeler et al., 2012), Rhabditis sp. (Wheeler et al., 2012), Mesorhabditis sp. (Wheeler et al., 2012), Acrobeloides sp. (Wheeler et al., 2012) and Pristionchus pacificus (Pires-daSilva and Sommer, 2004), as well as the animal-parasitic nematodes Haemonchus contortus (Geldhof et al., 2006), Trichostrongylus colubriformis (Issa et al., 2005) and Ostertagia ostertagi (Visser et al., 2006) exhibit a lower or variable sensitivity to (environmental) RNAi. Plant-parasitic nematodes generally seem to be susceptible to environmental RNAi, although variability in gene silencing efficiency has been observed during in planta experiments (Charlton et al., 2010; Huang et al., 2006; Ibrahim et al., 2011; Klink et al., 2009; Li et al., 2010; Lilley et al., 2012; Patel et al., 2010; Patel et al., 2008; Sindhu et al., 2009; Steeves et al., 2006; Urwin et al., 2002). Furthermore, researchers found that Brugia malayi is competent for environmental RNAi, but has no homologs of sid-1 and sid-2 in its genome (Aboobaker and Blaxter, 2004; Ghedin et al., 2009). In the parasitic nematode Globodera pallida, neuronal cells are susceptible to exogenously supplied dsRNA, while this is not the case for most neuronal cells in C. elegans (Kimber et al., 2007; Whangbo and Hunter, 2008).

This led researchers to investigate the differences between these so-called RNAi effectors in C. elegans and in other nematode species. An extensive study by Dalzell et al. (2011) looked into the available genomic and transcriptomic data of 13 nematode species and revealed that C. elegans possesses an expanded repertoire of RNAi-related genes compared to many other nematodes. Most species which were included in the investigation, except those belonging to the Caenorhabditis genus, contained less than half of the genes considered involved in RNAi in $C$. elegans. Furthermore, several components which are known to be involved in cellular uptake in C. elegans were found absent in parasitic nematodes (Dalzell et al., 2011). Most striking was the observation that sid-1 and sid-2 are absent in most investigated nematodes not belonging to the Caenorhabditis genus, including the freeliving nematode $P$. pacificus, the plant-parasitic Meloidogyne species and the human parasite B. malayi. Since both genes are instrumental in environmental RNAi and systemic spread in $C$. elegans, their absence might, at least partially, explain the lower sensitivity to RNAi in $P$. pacificus (Dalzell et al., 2011; Viney and Thompson, 2008). Interestingly though, several studies have shown successful systemic RNAi in Meloidogyne and Globodera species (Antonino de Souza Junior et al., 2013; Bakhetia et al., 2005; Dalzell et al., 2010a; Dalzell et al., 2010b; Kimber et al., 2007; Rosso et al., 2005) and successful environmental RNAi in B. malayi (Aboobaker and Blaxter, 2004; Song et al., 2010). This raises the question whether the loss of sid-2 and its role in cellular uptake from the gut

The present document has been produced and adopted by the bodies identified above as authors. This task has been carried out exclusively by the author(s) in the context of a contract between the European Food Safety Authority and the authors, awarded following a tender procedure. The present document is published complying with the transparency principle to which the Authority is subject. It may not be considered as an output adopted by the Authority. The European Food Safety Authority reserves its rights, view and position as regards the issues addressed and the conclusions reached in the present document, without prejudice to the rights of the authors. 
environment is compensated by other proteins or whether these genes are fast evolving and species have developed alternative pathways for sRNA uptake and systemic spread. Dalzell et al. (2011) did report that all nematodes used in the study displayed similar coverage of the functional protein groups in the entire RNAi machinery, meaning they possessed genes from each functional group. The main difference was a further expansion and diversification, possibly due to gene duplication events, within these functional groups in Caenorhabditis species, and notably in C. elegans.

Within the group of Caenorhabditis species, the differences were not as profound as compared to other nematode species, especially concerning genes that are involved in cellular uptake and transport processes (Dalzell et al., 2011). However, evidence for a fast evolution of some components can be found within this Caenorhabditis genus as well. Caenorhabditis briggsae was found to possess a highly divergent homolog of sid-2 (Cbsid-2), causing this species to lose its oral RNAi sensitivity. Their study showed that expression of Cesid-2 in C. briggsae caused C. briggsae to become sensitive again for oral RNAi. Conversely, expression of Cbsid-2 in sid-2 mutants of $C$. elegans could not rescue the response/sensitivity to oral RNAi sensitivity (Winston et al., 2007).

\section{Influence of the SRNA molecule on cellular uptake and systemic RNAi efficiency}

Most of the knowledge on cellular uptake mechanisms and systemic transport of sRNAs in C. elegans which is discussed above, was discovered in dsRNA feeding assays, often using bacterial-based delivery constructs. Data on cellular uptake of miRNA in nematodes is scarcer. Furthermore, the length of the dsRNA also proved to be a determining factor for environmental and systemic RNAi. In this section, we discuss what is known on uptake and transport of miRNA and on the influence of dsRNA/siRNA length on efficient environmental and systemic RNAi.

\section{Uptake and transport of miRNAs in nematodes}

miRNAs are small (20-25 nt) RNA molecules, first discovered in C. elegans, which are important in internal gene regulation in all animals (Lau et al., 2001). For the most part, they require an RNAi pathway which is molecularly distinct from the dsRNA/siRNA pathway (Hoogstrate et al., 2014; Parry et al., 2007). While most miRNAs seem to be intracellular, some have been found in extracellular biofluids inside extracellular vesicles, where they can potentially act as signalling molecules. Furthermore, some human parasitic nematodes have also been found to secrete these miRNAcontaining vesicles in their host (Buck et al., 2014; Coakley et al., 2015; Grainger et al., 2010; Maizels et al., 2012; Quintana et al., 2016). How these vesicles are then taken up by the recipient cells is not yet known. In humans, several hypotheses have been proposed, including endocytosis and membrane fusion (Boon and Vickers, 2013). These findings show that nematode cells are at least capable of exporting miRNAs, through the formation of these extracellular vesicles.

Regarding potential uptake of exogenous miRNA, not much is known in nematodes. An RNAi screening study to identify genes involved in the miRNA pathway did not identify any genes that are clearly related to uptake or extracellular transport. The authors did identify genes that are involved in intracellular trafficking and export from the nucleus (Parry et al., 2007), but whether they also have a role in import or export of the cell is unclear. Finally, while Shih and Hunter (2011) discovered that Sid-1 in C. elegans is capable of importing pre-miRNAs when expressed in S2 cells, we have found no evidence for oral or environmental miRNA uptake by nematodes.

\section{Length of SRNA}

The influence of dsRNA length on RNAi efficiency in nematodes was reported early in RNAi research. In 2000, Parrish et al. observed that in green fluorescent protein (GFP) construct carrying transgenic C. elegans, 717 bp-long double-stranded GFP (dsGFP) caused a stronger silencing effect of the GFP construct expression than 50-200 bp-long dsGFP (Parrish et al., 2000). At that moment, the reason for this was still unclear. Furthermore, Feinberg and Hunter (2003) suggested that this factor might be linked to the cellular uptake of dsRNA, and more specifically to Sid-1, important in systemic dsRNA transport. They found that for a $100 \mathrm{bp}$-long dsRNAs to have the same silencing efficiency as $500 \mathrm{bp}$ -

The present document has been produced and adopted by the bodies identified above as authors. This task has been carried out exclusively by the author(s) in the context of a contract between the European Food Safety Authority and the authors, awarded following a tender procedure. The present document is published complying with the transparency principle to which the Authority is subject. It may not be considered as an output adopted by the Authority. The European Food Safety Authority reserves its rights, view and position as regards the issues addressed and the conclusions reached in the present document, without prejudice to the rights of the authors. 
long dsRNA in Drosophila S2 cells expressing the C. elegans sid-1 gene, a 100-1000-fold higher concentration was necessary. Similarly, 21 bp siRNA required a $10^{5}$-fold higher concentration to have the same silencing efficiency as 100 bp-long dsRNA (Feinberg and Hunter, 2003). However, it was later found that Sid-1 does not have selectivity for length (Shih et al., 2009). In contrast, Sid-2, which is required for environmental uptake in the gut of $C$. elegans, does exhibit selectivity for dsRNA length and that dsRNAs longer than 25 bp are required for efficient uptake (McEwan et al., 2012).

In other nematodes, siRNA/dsRNA molecules ranging from $21 \mathrm{bp}$ to $1799 \mathrm{bp}$ have been shown to be capable of inducing functional dsRNA (Bakhetia et al., 2007; Dalzell et al., 2010b; Huang et al., 2006; Hussein et al., 2002; Urwin et al., 2002). Interestingly, when dsRNA of 1799 bp was compared to dsRNA of $204 \mathrm{bp}$ in an RNAi study on Nippostrongylus brasiliensis, researchers found that the silencing effect after feeding on the 1799 bp dsRNA only lasted 1-2 days, while the effect after exposure to 204 bp long dsRNA lasted up to at least 6 days (Hussein et al., 2002). However, to date, no comprehensive study on the correlation between length and RNAi efficiency has been performed in these non- $C$. elegans nematodes.

\subsubsection{Arthropods}

\section{Drosophila}

After C. elegans in 1998, D. melanogaster became the second animal species to have its genome sequenced in 2000. Both species henceforth became model species for many molecular studies, including RNAi. Experiments with S2 cells, derived from a primary D. melanogaster embryo cell line, had already shown that cell-autonomous RNAi and cellular uptake from medium is fully functional (Clemens et al., 2000; Worby et al., 2001). The first attempts to trigger RNAi in flies, using transgenic flies containing a transgenic RNAi construct, suggested that RNAi in D. melanogaster may not be systemic (Giordano et al., 2002; Kalidas and Smith, 2002). This was later confirmed by Roignant et al. (2003) who demonstrated, using a transgenic RNAi construct, that effective gene silencing was only achieved in cells in which RNAi was triggered. In contrast, research on transmission of viruses in $D$. melanogaster suggests that there must be a systemic RNAi pathway, at least for viral RNA (Saleh et al., 2009). These authors hypothesized that, while sRNA might not be exported from healthy, uninfected cells, virus-infected cells could release sRNA either after apoptosis or through a virusinduced shedding mechanism which can then be taken up by other cells in the organism (Saleh et al., 2009).

Additionally, various experimental designs attempting feeding RNAi in Drosophila species showed a lack of silencing response when naked dsRNA was administered (Taning et al., 2016; Whyard et al., 2009). However, when a transfection agent such as Lipofectamine was added to the dsRNA solution, RNAi silencing was observed (Taning et al., 2016; Whyard et al., 2009). These results indicated that besides a lack of systemic RNAi, Drosophila also seems to lack an efficient uptake of dsRNA from the midgut.

Interestingly, the Drosophila genome contains no homologs of the $C$. elegans sid genes, which, as discussed earlier, are instrumental in cellular uptake and systemic spread of dsRNA in C. elegans. However, in vivo experiments delivering dsRNA via microinjection in Drosophila had been shown to cause successful silencing in different tissues, suggesting there is dsRNA-uptake in various cell types in D. melanogaster (Dzitoyeva et al., 2001a; Dzitoyeva et al., 2001b; Dzitoyeva et al., 2003; Goto et al., 2003). In 2006, two independent studies which looked into the cellular uptake mechanism of dsRNA in S2 cells were published almost simultaneously. Both demonstrated that receptor-mediated endocytosis is involved in dsRNA uptake in S2 cells (Saleh et al., 2006b; Ulvila et al., 2006). The first, by Ulvila et al. (2006) used an RNAi-induced lethality screen to investigate components of the RNAi pathway in Drosophila and found that silencing clathrin heavy chain, a protein required for endocytosis, was able to rescue the cells and thus inhibit RNAi. This suggested that the cellular uptake of dsRNA in S2 cells might be facilitated by clathrin-mediated endocytosis. This hypothesis was confirmed by fluorescence microscopy experiments which showed that the fluorescently-labeled

The present document has been produced and adopted by the bodies identified above as authors. This task has been carried out exclusively by the author(s) in the context of a contract between the European Food Safety Authority and the authors, awarded following a tender procedure. The present document is published complying with the transparency principle to which the Authority is subject. It may not be considered as an output adopted by the Authority. The European Food Safety Authority reserves its rights, view and position as regards the issues addressed and the conclusions reached in the present document, without prejudice to the rights of the authors. 
dsRNA were internalized by S2 cells via cytoplasmic vesicles and by the fact that RNAi-mediated knockdown of the genes encoding the Scavenger receptor (SR-CI) and Eater inhibited endocytosis of dsRNA, proving that dsRNA uptake in S2 cells happens via scavenger receptor-mediated endocytosis (Ulvila et al., 2006). Scavenger receptor and eater are two structurally similar membrane-bound proteins which are known to be involved in phagocytosis of bacterial pathogens and are thus not dsRNA-specific (Kocks et al., 2005; Rämet et al., 2001; Ulvila et al., 2006). Interestingly though, expression of both $\mathrm{Sr}$-ci and eater in post-embryonic stages was found to be restricted mainly to haemolymph plasmatocytes of fruit flies, meaning that uptake in other cell types may use other pathways (Kocks et al., 2005; Kroeger et al., 2012; Pearson et al., 1995; Rämet et al., 2001).

The second 2006 study reporting on a dsRNA uptake mechanism in S2 cells was published by Saleh et al. (2006b). They also demonstrated the role of endocytosis in dsRNA uptake in S2 cells through fluorescence microscopy and the use of pharmacological endocytosis-inhibitors and identified an extended list of genes involved in this process using a genome-wide screen, as we have discussed earlier (See 4.1). Furthermore, adding inhibitors of Scavenger receptor to the medium also caused inhibition of the RNAi response, confirming that, at least in S2 cells, SR-CI is involved in uptake. Genes identified in the Saleh et al. (2006b) study are listed in Table 7 and include some elements which are also implicated in $C$. elegans cellular uptake of dsRNA.

Table 7: $\quad$ Overview of all genes implicated in cellular uptake of dsRNA in D. melanogaster S2 cells (adapted from Saleh et al., (2006b))

\begin{tabular}{lcc}
\hline Functional group & Drosophila gene ID & Drosophila gene name \\
\hline Proton transport & CG3161 & Vha16 \\
Vesicle mediated transport & CG17332 & VhaSFD \\
& CG9012 & Clathrin hc \\
& CG7057 & AP-50 \\
Intracellular transport & CG5915 & Rab7 \\
& CG6025 & Arf72A \\
& CG54125 & ninaC \\
& CG6177 & ldICp \\
Lipid metabolism & CG3248 & \\
& CG3911 & light \\
Proteolysis and peptidolysis & CG18028 & Gmer \\
& CG3495 & Pi3K59F \\
& CG5373 & Saposin-r \\
Other & CG12070 & \\
\hline
\end{tabular}

The present document has been produced and adopted by the bodies identified above as authors. This task has been carried out exclusively by the author(s) in the context of a contract between the European Food Safety Authority and the authors, awarded following a tender procedure. The present document is published complying with the transparency principle to which the Authority is subject. It may not be considered as an output adopted by the Authority. The European Food Safety Authority reserves its rights, view and position as regards the issues addressed and the conclusions reached in the present document, without prejudice to the rights of the authors. 


\section{Other arthropods}

As was the case with $C$. elegans, Drosophila does not seem to be the most representative species for other arthropods, in relation to cellular uptake of sRNAs and systemic RNAi. Current evidence suggests that Drosohila is more of an exception, rather than the rule for systemic uptake of RNAi. For example, systemic RNAi has been observed in several insect species, including Orthoptera (Dabour et al., 2011; Dong and Friedrich, 2005; Luo et al., 2013; Luo et al., 2012; Mito et al., 2008; Ronco et al., 2008; Santos et al., 2014; Wynant et al., 2012), Coleoptera (Bucher et al., 2002; Li et al., 2016; Miller et al., 2012; Powell et al., 2017; Tomoyasu et al., 2008; Zhu et al., 2011), Hemiptera (Araujo et al., 2006; Liu and Kaufman, 2004a; Liu and Kaufman, 2004b; Pitino et al., 2011) and Hymenoptera (Aronstein et al., 2006; Jarosch and Moritz, 2011; Lynch and Desplan, 2006). Further evolved lepidopteran and dipteran species are generally thought to be less efficient for systemic RNAi. However, feeding of RNAi leading to effects in other tissues than the gut have been reported for some Lepidoptera (Tian et al., 2009; Turner et al., 2006; Zhang et al., 2015) and Diptera (Li et al., 2011). The first indications for functional systemic RNAi systems have also been reported for several non-insect arthropod species, including several tick and mite species (Aung et al., 2011; Khila and Grbic, 2007; Kyaw Min et al., 2011; Wu and Hoy, 2014), as well as the crustacean Daphnia (Schumpert et al., 2015). Additionally, inducing RNAi by feeding, whether systemically or restricted to gut epithelial cells, has been achieved for many arthropod species, including insects, chelicerates and crustaceans (See Section 5.3).

\section{Sil-mediated uptake}

In contrast to Drosophila and some other dipterans, sid-1 homologues have been found in various other insect species, including T. castaneum (Tomoyasu et al., 2008; Xiao et al., 2015), L. decemlineata (Cappelle et al., 2016), D. virgifera (Miyata et al., 2014), B. mori (Tomoyasu et al., 2008), S. gregaria (Wynant et al., 2014a), L. migratoria (Luo et al., 2012), A. mellifera (Aronstein et al., 2006), N. lugens (Xu et al., 2013). Interestingly, beetle species possess multiple distinct sid-1 homologues (Cappelle et al., 2016; Miyata et al., 2012; Tomoyasu et al., 2008; Xiao et al., 2015). Given the highly efficient systemic RNAi in beetles, compared to other insects, it is tempting to hypothesize that this efficiency might be linked to an augmented repertoire of sid-1 genes. Several studies have been published in various species investigating the role of these sid-1 homologues in insects. A study by Tomoyasu et al. in 2008, investigating systemic RNAi in the red flour beetle $T$. castaneum, found that knockdown of these Tcsid-1 genes did not affect systemic RNAi in $T$. castaneum, suggesting they might not have the same involvement in SRNA cellular uptake as they have in $C$. elegans. Furthermore, a more detailed analysis of these gene sequences revealed that they share more similarity to tag-130, a C. elegans sid-1-like gene, than to sid-1 itself. Hence, they decided to name these genes sid-1-like ( no involvement in RNAi (Tomoyasu et al., 2008). Another observation casting doubt over the link between sil gene repertoire and efficient environmental or systemic RNAi, was the fact that several lepidopteran species, which are much less sensitive to (systemic) RNAi (Terenius et al., 2011), also contain three sil genes, including B. mori, Danaus plexipus and Spodoptera littoralis (Cappelle et al., 2016; Tomoyasu et al., 2008). Of course, a lack of sensitivity to systemic or environmental RNAi could be due to several factors. One example is degradation of dsRNA in digestive systems and/or haemolymph of insect species (Allen and Walker, 2012; Arimatsu et al., 2007; Christiaens et al., 2014; Garbutt et al., 2013; Liu et al., 2012b; Wynant et al., 2014b) which seems especially important in Lepidoptera.

More recently, studies on SRNA cellular uptake and systemic RNAi mechanisms have been conducted in several other insect species. In the coleopteran species $D$. virgifera and $L$. decemlineata, two sil genes were identified in the genome and, in contrast to $T$. castaneum, these sil genes were found to be required for efficient environmental or systemic RNAi in both species (Cappelle et al., 2016; Miyata et al., 2014). In the $D$. virgifera study, a two-step in vivo assay was used to investigate the effect of knocking down silA and silC on RNAi efficiency. Miyata et al. (2014) observed only a modest decrease

The present document has been produced and adopted by the bodies identified above as authors. This task has been carried out exclusively by the author(s) in the context of a contract between the European Food Safety Authority and the authors, awarded following a tender procedure. The present document is published complying with the transparency principle to which the Authority is subject. It may not be considered as an output adopted by the Authority. The European Food Safety Authority reserves its rights, view and position as regards the issues addressed and the conclusions reached in the present document, without prejudice to the rights of the authors. 
in RNAi efficiency after either silA or silC were silenced. The limited effect could just be due to the fact that only a partial knockdown of the effector genes was achieved $(50-60 \%)$, but it could also indicate that these sils are both involved in the uptake mechanism and could exhibit some redundancy towards each other. Another possible explanation is that multiple distinct pathways are involved in uptake of dsRNA in D. virgifera. In the L. decemlineata study, Cappelle et al. (2016) also found a small effect on RNAi feeding efficiency after silencing of silA (14.4\% rescue of expression) or silC (8.1\% rescue of expression), but they also looked at the effect of simultaneous silencing and found a $24.1 \%$ rescue of the reporter gene expression upon simultaneous silencing of both sil genes. In this study, knockdown of silA and silC only resulted in a $59 \%$ and $66 \%$ gene silencing, respectively, making it difficult to draw conclusions on the importance of these genes for environmental or systemic RNAi.

In non-beetle species, sil has been suggested to be involved in environmental RNAi in the honeybee A. mellifera and the brown planthopper $N$. lugens, both containing one sil gene. In the honeybee, Aronstein et al. (2006) observed that upon feeding of dsRNA, honeybee sil expression increased 3.4fold compared to the control group which was not fed dsRNA While the authors concluded that this is proof for involvement of sil in cellular uptake or transport of the silencing signal, the evidence is indirect at best. Further research in honeybee sil is needed to confirm this hypothesis. In N. lugens, the evidence for sil involvement is stronger. The authors injected dsRNA specific for $N$. lugens sil, together with dsRNA targeting distal-less ( $d I I)$, a gene which is involved in development of the distal limb structure. Silencing of dll causes claw defects in this planthopper. Injection of dsSil led to strong silencing of sil throughout the body ( $>90 \%$ ) on day 3 and at day 6 , the authors observed normal claw structures for most treated insects, while only a few had minor defects (Xu et al., 2013). In contrast, even though sil genes were discovered in the genomes of the orthopteran locusts $S$. gregaria and $L$. migratoria, knockdown of these genes had no adverse effect on RNAi efficiency, suggesting that they might not play an important role for dsRNA uptake (Luo et al., 2012; Wynant et al., 2014a). A full overview of what is known so far on sil-involvement in insect RNAi is presented in Table 8.

\section{Endocytosis-mediated uptake}

As discussed above, even though sil genes are not present in Drosophila, S2 cells are still capable of internalizing dsRNA efficiently due to the scavenger receptor-mediated endocytosis of dsRNA. In recent years, a number of studies have investigated whether this pathway also plays a role in other insects and found proof for involvement of this pathway in feeding or systemic RNAi in every species in which it was investigated (Table 8), including $T$. castaneum, L. decemlineata and S. gregaria (Cappelle et al., 2016; Wynant et al., 2014a; Xiao et al., 2015). In all three studies, so-called RNAi of RNAi experiments were used to implicate key endocytosis components in RNAi efficiency. Interestingly, Cappelle et al. (Cappelle et al., 2016) showed for the first time that both endocytosis and sil genes play a role in uptake or transport of dsRNA in an insect species, namely $L$. decemlineata. They found that silencing chc and vha16, both important members of the endocytosis pathway, impaired the feeding RNAi efficiency significantly. The observed effect was also more profound than when silA and silC were silenced, indicating that endocytosis might be more important in dsRNA uptake. Using a pharmacological inhibitor of endocytosis, there was a near-complete rescue of reporter gene expression (Cappelle et al., 2016). Recently, endocytosis was also shown to be involved in RNAi in a non-insect arthropod, namely the tick H. longicornis (Aung et al., 2011). In addition, this study used an RNAi approach to investigate the impact of Scavenger Receptor B (SR-B) silencing on the silencing efficiency of the reporter genes, in this case vitellogenin-1 $(V g-1)$ and vitellogenin receptor $(V g R)$. Knockdown of $S R-B$ before $V g-1$ or $V g R$ silencing led to rescue of the hatching rate to $83 \%$, compared to $0 \%$ and $13.7 \%$ after just $V g-1$ or $V g R$ silencing, respectively (Aung et al., 2011).

\section{Conclusions on arthropods}

This study of the literature indicates that dsRNA uptake in insects, and possibly in other arthropods, is distinct from uptake mechanisms in nematodes, or in $C$. elegans, more specifically. The available

The present document has been produced and adopted by the bodies identified above as authors. This task has been carried out exclusively by the author(s) in the context of a contract between the European Food Safety Authority and the authors, awarded following a tender procedure. The present document is published complying with the transparency principle to which the Authority is subject. It may not be considered as an output adopted by the Authority. The European Food Safety Authority reserves its rights, view and position as regards the issues addressed and the conclusions reached in the present document, without prejudice to the rights of the authors. 
evidence suggests that Sid-1-like transmembrane channels might play some role in dsRNA uptake and transport in insects, but the proof for this is not unequivocal. While Sil has been implicated in $N$. lugens, D. virgifera and $L$. decemlineata, it did not appear to be necessary for systemic RNAi in $T$. castaneum and the orthopteran S. gregaria and L. migratoria. Perhaps there is a link with environmental RNAi here, as the species in which Sil has not been considered necessary are also not sensitive to environmental RNAi, while most insects for which Sil was found to be required for efficient systemic RNAi also exhibit a robust environmental RNAi response. One exception is $N$. lugens, for which feeding RNAi is not successful. But, as suggested before, other factors such as dsRNA stability in the digestive system could also play a role here. The studies conducted so far seem to indicate that endocytosis might play a more prominent role than sil-mediated uptake or transport of dsRNA. However, it cannot be excluded that both pathways act together and could have a synergistic effect (Cappelle et al., 2016).

The present document has been produced and adopted by the bodies identified above as authors. This task has been carried out exclusively by the author(s) in the context of a contract between the European Food Safety Authority and the authors, awarded following a tender procedure. The present document is published complying with the transparency principle to which the Authority is subject. It may not be considered as an output adopted by the Authority. The European Food Safety Authority reserves its rights, view and position as regards the issues addressed and the conclusions reached in the present document, without prejudice to the rights of the authors. 
Table 8: Overview of reported dsRNA uptake experiments in arthropods. Table adapted from Cappelle et al. (2016)

\begin{tabular}{|c|c|c|c|c|c|c|c|c|}
\hline Insect order & Species & $\begin{array}{l}\text { Environmental } \\
\text { RNAi }\end{array}$ & $\begin{array}{l}\text { Systemic } \\
\text { RNAi }\end{array}$ & $\begin{array}{l}\text { No. of sid-1 } \\
\text { homologs } \\
\text { present }\end{array}$ & $\begin{array}{l}\text { Application } \\
\text { method }\end{array}$ & $\begin{array}{l}\text { SID-1 is } \\
\text { involved }\end{array}$ & $\begin{array}{l}\text { Endocytosis } \\
\text { is involved }\end{array}$ & Reference \\
\hline \multirow[t]{2}{*}{ Diptera } & $\begin{array}{l}\text { Drosophila melanogaster } \\
\text { (S2 cell line) }\end{array}$ & + & + & 0 & $\mathrm{~S}$ & no & yes & $\begin{array}{l}\text { (Saleh et al., 2006b; Ulvila et } \\
\text { al., 2006) }\end{array}$ \\
\hline & Bactrocera dorsalis & + & + & 0 & $\mathrm{~F}$ & no & yes & (Li et al., 2015c) \\
\hline \multirow[t]{3}{*}{ Coleoptera } & Tribolium castaneum & + & ++ & 3 & I & no & yes & $\begin{array}{l}\text { (Tomoyasu et al., 2008; Xiao } \\
\text { et al., 2015) }\end{array}$ \\
\hline & Diabrotica virgifera & ++ & ++ & 2 & $\mathrm{~F}$ & yes & n.d. & (Miyata et al., 2014) \\
\hline & $\begin{array}{l}\text { Leptinotarsa } \\
\text { decemlineata }\end{array}$ & ++ & ++ & 2 & $\mathrm{~F}$ & yes & yes & (Cappelle et al., 2016) \\
\hline Lepidoptera & Bombyx mori & - & + & 3 & I & no & n.d. & (Tomoyasu et al., 2008) \\
\hline \multirow[t]{2}{*}{ Orthoptera } & Schistocerca gregaria & - & ++ & 1 & I & no & yes & (Wynant et al., 2014a) \\
\hline & Locusta migratoria & - & ++ & 1 & I & no & n.d. & (Luo et al., 2012) \\
\hline Hymenoptera & Apis mellifera & + & + & 1 & $\mathrm{~F}$ & yes & n.d. & (Aronstein et al., 2006) \\
\hline Hemiptera & Nilaparvata lugens & - & + & 1 & I & yes & n.d. & (Xu et al., 2013) \\
\hline Ixodida & $\begin{array}{l}\text { Haemaphysalis } \\
\text { longicornis }\end{array}$ & $+*$ & $+*$ & $?$ & I & n.d. & yes & (Aung et al., 2011) \\
\hline
\end{tabular}

++ : present and robust, +: present but not robust, -: not present, F: feeding, I: injection, S: soaking, n.d.: not determined.

* Assessments were based on a single soaking experiment and only a small number of injection experiments available for this tick species

The present document has been produced and adopted by the bodies identified above as authors. This task has been carried out exclusively by the author(s) in the context of a contract between the European Food Safety Authority and the authors, awarded following a tender procedure. The present document is published complying with the transparency principle to which the Authority is subject. It may not be considered as an output adopted by the Authority. The European Food Safety Authority reserves its rights, view and position as regards the issues addressed and the conclusions reached in the present document, without prejudice to the rights of the authors. 
Furthermore, as shown in Table 8, several of the studies used injection as a delivery method, making it hard to draw conclusions as to the necessity of Sil proteins in feeding RNAi, or even systemic RNAi. Indeed, several of the studies reporting systemic RNAi were actually performed using an injection delivery into the body cavity or haemolymph, so that systemic RNAi has not been clearly demonstrated. They showed that dsRNA can spread through the haemolymph to different tissues, but not necessarily that the silencing signal can be exported from one cell and imported into another. Further research will be necessary to unravel the uptake mechanisms in insects and to investigate why there is such variability in feeding and systemic RNAi in insects.

\subsubsection{Annelids and molluscs}

None of the studies described in Annex 2 on use of RNAi in annelids and molluscs reported mechanisms of cellular uptake or systemic transport of dsRNA. In total, three studies were retrieved reporting successful oral or soaking RNAi in molluscs (Chen et al., 2014; Knight et al., 2011; Wang et al., 2016), suggesting that snails and clams might possess an environmental RNAi capacity. No feeding RNAi studies for any annelid species has been found in the systematic literature searches, nor in subsequent manual searches.

\subsubsection{Amplification of the silencing signal}

For a strong systemic RNAi response, a sufficient number of siRNAs is logically required inside the body of the target animal, in order to invoke a silencing response in a sufficiently high number of cells. While this could be achieved by a constant supply of sRNAs through feeding (e.g., in planta), strong systemic RNAi responses are also observed in some species upon a single delivery of a small amount of these sRNAs. In C. elegans, a pathway has been identified causing an amplification of the silencing signal inside the body of these nematodes, similar to the one described in plants earlier (Alder et al., 2003; Pak and Fire, 2007; Sijen et al., 2001; Sijen et al., 2007; Smardon et al., 2000; Tsai et al., 2015; Zhang et al., 2012). In short, this mechanism is based on RNA-dependant RNA polymerases (RdRPs) which are able to produce secondary siRNAs from the mRNA that is being targeted by the primary siRNAs. Sijen et al. (2007) described these secondary siRNAs as being distinct from primary siRNAs in the sense that they contain di- or triphosphates at the $5^{\prime}$ end, while primary siRNAs have a monophosphate group at the $5^{\prime}$ end. Furthermore, the secondary siRNAs were found to be of antisense polarity only. They also showed that these siRNAs were not produced directly from the miRNA or exogenous dsRNA, but from the targeted mRNA, since single nucleotide mismatches in the primary siRNAs are not present anymore in the secondary siRNAs, who regain the full homology with the target mRNA (Sijen et al., 2007).

Pak and Fire, (2007) reported that these secondary siRNAs constitute the vast majority of siRNAs present in C. elegans, indicating its importance in RNAi-mediated gene silencing efficiency in these nematodes. Furthermore, since these secondary siRNAs are synthesized from the targeted mRNA, an important consequence of this mechanism is the occurrence of so-called transitive RNAi, which entails silencing regions of a mRNA which were originally not covered by the original dsRNA (Alder et al., 2003; Sijen et al., 2001; Sijen et al., 2007). Alder et al. (2003) confirmed the existence of this transitive RNAi in C. elegans by injecting dsRNA specific to GFP in mutant worms which contained a homologous $g f p$ sequence fused to the sequence of a number of essential genes. The dsGFP resulted in silencing of these essential genes. In $C$. elegans, secondary siRNAs were found to mainly cover the region of the mRNA that is upstream of the silencing trigger (Alder et al., 2003; Pak and Fire, 2007; Sijen et al., 2001). Interestingly, siRNAs also seem to induce a so-called Slicer activity causing them to cleave target mRNAs much more efficiently than primary siRNAs. So enhanced RNAi efficiency is not only caused by the increased amount of siRNAs present, but also by a more efficient target mRNA cleavage (Aoki et al., 2007).

The exact mechanism of secondary siRNA production has not been elucidated yet. The main actors in this pathway appear to be complexes containing RRF-1 or EGO-1, which are RdRPs able to produce

The present document has been produced and adopted by the bodies identified above as authors. This task has been carried out exclusively by the author(s) in the context of a contract between the European Food Safety Authority and the authors, awarded following a tender procedure. The present document is published complying with the transparency principle to which the Authority is subject. It may not be considered as an output adopted by the Authority. The European Food Safety Authority reserves its rights, view and position as regards the issues addressed and the conclusions reached in the present document, without prejudice to the rights of the authors. 
secondary siRNAs in a Dicer-independent manner (Aoki et al., 2007). RRF-1 seems to create siRNAs in an unprimed manner using as a template $3^{\prime}$ uridylated mRNA fragments produced by an argonaute enzyme called RDE-8 (Tsai et al., 2015). One more RdRP that has been identified is RRF-3. However, this protein seems to exert an inhibitory role on secondary siRNA production (Simmer et al., 2002). Furthermore, Zhang et al. (2012) identified several other genes involved in this amplification mechanism, including rde-10, rde-11, rsd-2, rsd-6 and haf-6. RDE-10 and RDE-11 proteins form a complex that appears to be essential for amplification of siRNAs. Indeed, mutation in these genes resulted in worms which remained sensitive to high doses of dsRNA, but lose their sensitivity upon delivery of small doses of RNAi (Zhang et al., 2012). Over the years, it also became clear that secondary siRNAs and their gene silencing require a distinct RNAi pathway than primary siRNAs, including different argonaute effectors, called secondary siRNA-specific argonautes (SAGOs) and worm-specific argonautes (WAGOs) (Tsai et al., 2015). While the RNAi pathways for exogenous dsRNA and endogenous miRNA are for the most part distinct in C. elegans, this RdRP pathway seems to largely overlap in both pathways. Indeed, RDE-10 and RDE-11 have been shown to be required for the production of secondary siRNAs of both endogenous and exogenous RNAi-triggering sRNAs (Zhang et al., 2012).

While RdRP activity seems to be a crucial factor in RNAi efficiency in C. elegans, little data is available on this mechanism in other nematodes. Dalzell et al. (Dalzell et al., 2011) found that the EGO-1 RdRP is present in most nematodes that they studied, including several non-Caenorhabditis species such as Ascaris suum, B. malayi, Meloidogyne sp., H. contortus, Oesophagostomum dentatum and P. pacificus (Dalzell et al., 2011). In contrast, the rrf-1 gene was absent in all but one non-Caenorhabditis species (Dalzell et al., 2011). The high conservation is perhaps not surprising, given that this RdRP plays a major role in endogenous RNAi processes including genome surveillance and germline development (Smardon et al., 2000). Dalzell et al. (2011) concluded that the presence of EGO-1 in most nematodes indicates that most species in this phylum are capable of at least some form of secondary siRNA production.

In arthropods, the presence of homologs for these $C$. elegans RdRPs in the genome has only been reported for two tick species, namely Rhipicephalus microplus and I. scapularis (Kurscheid et al., 2009), which both contain an EGO-1 homolog in their genome and in the two-spotted spidermite Tetranychus urticae, which contains 5 RdRP gene copies (Grbić et al., 2011). However, the activity of these RdRPs and possible involvement in the chelicerate RNAi pathway is still to be confirmed. So far, no homologs have been reported in insect or crustacean species. Whether this means that insects completely lack any amplification system is not sure. Based on the sensitivity of some species for environmental, systemic and sometimes even parental RNAi, notably in a number of beetle species, it would not be surprising to find that some insects have a similar but distinct mechanism by which the RNA-silencing signal can be amplified. However, no evidence for such a pathway has been reported.

\subsubsection{Parental RNAi}

Parental RNAi (pRNAi) or heritable RNAi, meaning RNAi-mediated gene silencing in the offspring of dsRNA-exposed adult females, has been observed in several invertebrate species, including the nematodes C. elegans (Alcazar et al., 2008; Grishok et al., 2000; Vastenhouw et al., 2006) and Meloidogyne chitwoodi (Dinh et al., 2014a; Dinh et al., 2014b), and several insect species belonging to different orders including the coleopterans T. castaneum (Bucher et al., 2002), D. virgifera (Khajuria et al., 2015), Orchesella cincta (Konopova and Akam, 2014), the orthopteran Gryllus bimaculatus (Mito et al., 2006), the hemipterans Oncopeltus fasciatus (Liu and Kaufman, 2004a), Rhodnius prolixus (Paim et al., 2013), Myzus persicae (Coleman et al., 2015), Acyrthosiphon pisum (Mao et al., 2013), the blattodean Blattella germanica (Piulachs et al., 2010) and the hymenopterans Athalia rosae (Yoshiyama et al., 2013) and Nasonia vitripennis (Lynch and Desplan, 2006).

In $C$. elegans, transgenerational silencing effects have been observed over multiple generations (Alcazar et al., 2008; Grishok et al., 2000a; Vastenhouw et al., 2006). Upon injection of dsRNA in the

The present document has been produced and adopted by the bodies identified above as authors. This task has been carried out exclusively by the author(s) in the context of a contract between the European Food Safety Authority and the authors, awarded following a tender procedure. The present document is published complying with the transparency principle to which the Authority is subject. It may not be considered as an output adopted by the Authority. The European Food Safety Authority reserves its rights, view and position as regards the issues addressed and the conclusions reached in the present document, without prejudice to the rights of the authors. 
parent, most RNAi effects remain visible in the next (F1) generation. The silencing effects typically recede over the generations eventually leading to a return to a normal phenotype. Interestingly, it was found that both males and females were able to pass the silencing signal on to the next generation in C. elegans so that both sperm and oocytes were responsible for transmitting the silencing signal (Alcazar et al., 2008). Furthermore, these studies also observed that the silencing signal is passed on unevenly between the progeny, leading to siblings with very different silencing effects (Alcazar et al., 2008; Vastenhouw et al., 2006). The exact mechanisms of this heritable silencing effect are still not fully understood. Grishok et al. (2000) observed that chromatine remodelling factors were necessary for a heritable silencing effect and that $r d e-2$ and $r d e-4$ were not required for successful heritable silencing, which suggested that while the initial silencing in the first (exposed) generation is due to RNAi, the inheritance mechanism could involve silencing at the transcriptional level by chromatin changes at the locus, rather than the post-transcriptional level (Vastenhouw et al., 2006).

Most of the pRNAi studies in arthropods involved heritable silencing effects upon injection of pupae or of the female adult. However, pRNAi has also been observed after feeding on dsRNA by the female adult. Khajuria et al. showed that feeding dsRNA specific to brahma and hunchback genes to adult female WCRs resulted in eggs that did not hatch, showing the involvement of both genes in embryonic development (Khajuria et al., 2015). Vélez et al. (2016) further investigated several parameters for successful pRNA in WCR, and demonstrated that pRNAi exhibits a dsRNA exposure dose- and duration dependant effect.

\subsubsection{Conclusions on uptake and systemic spread}

In this review we have provided an extensive overview of the available literature regarding mechanisms of cellular uptake and systemic spread of sRNAs in invertebrates. By far the most information on these mechanisms and pathways is available in nematodes, and more specifically the free living C. elegans. This nematode possesses a high sensitivity for feeding RNAi, due to a welldeveloped system for cellular uptake, systemic spread and an amplification system which produces secondary siRNAs. Furthermore, the silencing signal in $C$. elegans can be observed over multiple generations. However, as has been clearly shown in the literature, a considerable degree of variation exists within nematodes concerning these pathways. Most nematodes outside of the genus of Caenorhabditis are missing several genes which are considered important in C. elegans, which may explain observed differences in RNAi efficiency in different nematode species. While there are other factors involved in explaining variability in RNAi sensitivity in invertebrates, which are discussed further in other sections, cellular uptake of the sRNA and systemic spread of the silencing signal have been shown to be critical mechanisms for environmental RNAi.

Even more differences and variability in terms of uptake and systemic spread pathways were found in arthropods, where it seems that cellular uptake is much less efficient, especially some insect orders such as Diptera and Lepidoptera. While arthropods do contain some elements of the elaborate cellular uptake and systemic spread systems found in C. elegans, their requirement and involvement in successful environmental or systemic RNAi has not yet been clearly demonstrated. What is clear is that, in insects and possibly some other taxonomic groups, cellular uptake is regulated by two pathways which may or may not be linked. The occurrence and robustness of systemic RNAi in arthropods is variable and no evidence for an amplification system of the silencing signal, as is present in C. elegans and many other nematodes, has been reported yet. However, given the efficiency of environmental and systemic RNAi in some insects, notably Coleoptera, and the fact that parental RNAi has been described for several arthropods, there may well be such a system in some arthropods.

Given the very small number of RNAi studies in molluscs and annelids, no information was found regarding the uptake mechanisms in these phyla. A number of feeding studies in clams and snails suggest that these molluscs possess some sort of environmental RNAi capacity, but future research is

The present document has been produced and adopted by the bodies identified above as authors. This task has been carried out exclusively by the author(s) in the context of a contract between the European Food Safety Authority and the authors, awarded following a tender procedure. The present document is published complying with the transparency principle to which the Authority is subject. It may not be considered as an output adopted by the Authority. The European Food Safety Authority reserves its rights, view and position as regards the issues addressed and the conclusions reached in the present document, without prejudice to the rights of the authors. 
required to investigate whether mechanisms similar to those in nematodes or arthropods are involved.

\subsubsection{References}

Aboobaker $\mathrm{AA}$ and Blaxter ML, 2004. Functional genomics for parasitic nematodes and platyhelminths. Trends in Parasitology 20, 178-184.

Akay A, Sarkies $P$ and Miska EA, 2015. E. coli OxyS non-coding RNA does not trigger RNAi in C. elegans. Scientific Reports, 5, 9597.

Alcazar RM, Lin R and Fire AZ, 2008. Transmission Dynamics of Heritable Silencing Induced by Double-Stranded RNA in Caenorhabditis elegans. Genetics 180, 1275-1288.

Alder MN, Dames S, Gaudet J and Mango SE, 2003. Gene silencing in Caenorhabditis elegans by transitive RNA interference. Rna-a Publication of the Rna Society 9, 25-32.

Allen ML and Walker WB III, 2012. Saliva of Lygus lineolaris digests double stranded ribonucleic acids. Journal of Insect Physiology 58, 391-396.

Antonino De Souza Junior JD, Coelho RR, Lourenco IT, et al., 2013. Knocking-down Meloidogyne incognita proteases by plant-delivered dsRNA has negative pleiotropic effect on nematode vigor. PLOS ONE 8, 1-17.

Aoki $\mathrm{K}$, Moriguchi $\mathrm{H}$, Yoshioka $\mathrm{T}$, Okawa $\mathrm{K}$ and Tabara $\mathrm{H}, 2007$. In vitro analyses of the production and activity of secondary small interfering RNAs in C-elegans. Embo Journal 26, 5007-5019.

Araujo RN, Santos A, Pinto FS, Gontijo NF, Lehane MJ and Pereira MH, 2006. RNA interference of the salivary gland nitrophorin 2 in the triatomine bug Rhodnius prolixus (Hemiptera: Reduviidae) by dsRNA ingestion or injection. Insect Biochemistry and Molecular Biology 36, 683-693.

Arimatsu Y, Furuno T, Sugimura $Y$, et al., 2007. Purification and properties of double-stranded RNAdegrading nuclease, dsRNase, from the digestive juice of the silkworm, Bombyx mori. Journal of Insect Biotechnology and Sericology 76, 57-62.

Aronstein K, Pankiw T and Saldivar E, 2006. SID-I is implicated in systemic gene silencing in the honey bee. Journal of Apicultural Research, 45, 20-24.

Aung KM, Boldbaatar D, Umemiya-Shirafuji $R$, et al., 2011. Scavenger receptor mediates systemic RNA interference in ticks. PLoS ONE 6, e28407.

Bakhetia M, Charlton W, Atkinson HJ, Mcpherson MJ, 2005. RNA interference of dual oxidase in the plant nematode Meloidogyne incognita. Molecular Plant-Microbe Interactions 18, 1099-1106.

Bakhetia M, Urwin PE, Atkinson HJ, 2007. qPCR analysis and RNAi define pharyngeal gland cellexpressed genes of Heterodera glycines required for initial interactions with the host. Molecular Plant-Microbe Interactions 20, 306-312.

Barrett JW, Brownwright AJ, Primavera MJ, Palli SR, 1998. Studies of the nucleopolyhedrovirus infection process in insects by using the green fluorescence protein as a reporter. Journal of Virology 72, 3377-3382.

Baum JA, Bogaert T, Clinton W, et al., 2007. Control of coleopteran insect pests through RNA interference. Nature Biotechnology 25, 1322-1326.

Bird AF and Bird J, 2012. The structure of nematodes. Academic Press.

Bonning BC and Chougule NP, 2014. Delivery of intrahemocoelic peptides for insect pest management. Trends in Biotechnology 32, 91-98.

The present document has been produced and adopted by the bodies identified above as authors. This task has been carried out exclusively by the author(s) in the context of a contract between the European Food Safety Authority and the authors, awarded following a tender procedure. The present document is published complying with the transparency principle to which the Authority is subject. It may not be considered as an output adopted by the Authority. The European Food Safety Authority reserves its rights, view and position as regards the issues addressed and the conclusions reached in the present document, without prejudice to the rights of the authors. 
Boon RA and Vickers KC, 2013. Intercellular transport of microRNAs. Arteriosclerosis, thrombosis, and vascular biology, 33, 186-192.

Bucher G, Scholten J and Klingler M, 2002. Parental RNAi in Tribolium (Coleoptera). Current Biology, 12, R85-R86.

Buck AH, Coakley G, Simbari F, et al., 2014. Exosomes secreted by nematode parasites transfer small RNAs to mammalian cells and modulate innate immunity. Nature Communications 5, 5488.

Calixto A, Chelur D, Topalidou I, Chen X and Chalfie M, 2010. Enhanced neuronal RNAi in C. elegans using SID-1. Nature Methods, 7, 554-559.

Cappelle K, De Oliveira CFR, Van Eynde B, Christiaens O and Smagghe G, 2016. The involvement of clathrin-mediated endocytosis and two Sid-1-like transmembrane proteins in doublestranded RNA uptake in the Colorado potato beetle midgut. Insect Molecular Biology, 25, 315-23.

Casartelli M, Corti P, Cermenati G, et al., 2007. Absorption of horseradish peroxidase in Bombyx mori larval midgut. Journal of Insect Physiology 53, 517-525.

Chan SY, Snow JW, 2017. Uptake and impact of natural diet-derived small RNA in invertebrates: Implications for ecology and agriculture. RNA biology 14, 402-14.

Charlton WL, Harel HYM, Bakhetia M, Hibbard JK, Atkinson HJ, Mcpherson MJ, 2010. Additive effects of plant expressed double-stranded RNAs on root-knot nematode development. International Journal for Parasitology 40, 855-64.

Chen $H$, Yang $X$, Tang $T$, et al., 2014. The involvement of cysteine-rich intestinal protein in early development and innate immunity of Asiatic hard clam, Meretrix meretrix. Fish \& Shellfish Immunology 40, 435-40.

Christiaens O, Smagghe G, 2014. The challenge of RNAi-mediated control of hemipterans. Current Opinion in Insect Science 6, 15-21.

Christiaens O, Swevers L, Smagghe G, 2014. DsRNA degradation in the pea aphid (Acyrthosiphon pisum) associated with lack of response in RNAi feeding and injection assay. Peptides 53, 307-14.

Clem RJ, Passarelli AL, 2013. Baculoviruses: sophisticated pathogens of insects. PLoS Pathog 9, e1003729.

Clemens JC, Worby CA, Simonson-Leff N, et al., 2000. Use of double-stranded RNA interference in Drosophila cell lines to dissect signal transduction pathways. Proceedings of the National Academy of Sciences of the United States of America 97, 6499-503.

Coakley G, Maizels RM, Buck AH, 2015. Exosomes and other extracellular vesicles: the new communicators in parasite infections. Trends in Parasitology 31, 477-89.

Coleman AD, Wouters RHM, Mugford ST, Hogenhout SA, 2015. Persistence and transgenerational effect of plant-mediated RNAi in aphids. Journal of Experimental Botany 66, 541-8.

Dabour N, Bando T, Nakamura T, et al., 2011. Cricket body size is altered by systemic RNAi against insulin signaling components and epidermal growth factor receptor. Development Growth \& Differentiation 53, 857-69.

Dalzell JJ, Mcmaster S, Fleming CC, Maule AG, 2010a. Short interfering RNA-mediated gene silencing in Globodera pallida and Meloidogyne incognita infective stage juveniles. International Journal for Parasitology 40, 91-100.

Dalzell JJ, Mcveigh P, Warnock ND, et al., 2011. RNAi Effector Diversity in Nematodes. Plos Neglected Tropical Diseases 5.

The present document has been produced and adopted by the bodies identified above as authors. This task has been carried out exclusively by the author(s) in the context of a contract between the European Food Safety Authority and the authors, awarded following a tender procedure. The present document is published complying with the transparency principle to which the Authority is subject. It may not be considered as an output adopted by the Authority. The European Food Safety Authority reserves its rights, view and position as regards the issues addressed and the conclusions reached in the present document, without prejudice to the rights of the authors. 
Dalzell J], Warnock ND, Stevenson MA, Mousley A, Fleming CC, Maule AG, 2010b. Short interfering RNA-mediated knockdown of drosha and pasha in undifferentiated Meloidogyne incognita eggs leads to irregular growth and embryonic lethality. International Journal for Parasitology 40, 130310.

Dinh PTY, Brown CR, Elling AA, 2014a. RNA Interference of Effector Gene Mc16D10L Confers Resistance Against Meloidogyne chitwoodi in Arabidopsis and Potato. Phytopathology 104, 1098106.

Dinh PTY, Zhang L, Brown CR, Elling AA, 2014b. Plant-mediated RNA interference of effector gene Mc16D10L confers resistance against Meloidogyne chitwoodi in diverse genetic backgrounds of potato and reduces pathogenicity of nematode offspring. Nematology 16, 669-82.

Dong Y, Friedrich M, 2005. Nymphal RNAi: systemic RNAi mediated gene knockdown in juvenile grasshopper. Bmc Biotechnology 5.

Dzitoyeva S, Dimitrijevic N, Manev H, 2001a. Intra-abdominal injection of double-stranded RNA into anesthetized adult Drosophila triggers RNA interference in the central nervous system. Molecular Psychiatry 6, 665-70.

Dzitoyeva SG, Dimitrijevic N, Manev H, 2001b. RNA interference method for functional gene knockout in adult Drosophila. Society for Neuroscience Abstracts 27, 2347-.

Dzitoyeva SG, Dimitrijevic N, Manev H, 2003. Identification of a novel Drosophila gene, beltless, using injectable embryonic and adult RNA interference. Society for Neuroscience Abstract Viewer and Itinerary Planner 2003, Abstract No. 325.9-Abstract No. .9.

Engelhard E, Kam-Morgan L, Washburn J, Volkman L, 1994. The insect tracheal system: a conduit for the systemic spread of Autographa californica M nuclear polyhedrosis virus. Proceedings of the National Academy of Sciences 91, 3224-7.

Fabioux C, Corporeau C, Quillien V, Favrel P, Huvet A, 2009. In vivo RNA interference in oyster -vasa silencing inhibits germ cell development. Febs Journal 276, 2566-73.

Fanelli E, Di Vito M, Jones JT, De Giorgi C, 2005. Analysis of chitin synthase function in a plant parasitic nematode, Meloidogyne artiellia, using RNAi. Gene 349, 87-95.

Feinberg EH, Hunter CP, 2003. Transport of dsRNA into cells by the transmembrane protein SID-1. Science 301, 1545-7.

Fiandra L, Casartelli M, Cermenati G, Burlini N, Giordana B, 2009. The intestinal barrier in lepidopteran larvae: permeability of the peritrophic membrane and of the midgut epithelium to two biologically active peptides. Journal of Insect Physiology 55, 10-8.

Fire A, Xu S, Montgomery MK, Kostas SA, Driver SE, Mello CC, 1998. Potent and specific genetic interference by double-stranded RNA in Caenorhabditis elegans. Nature (London) 391, 806-11.

Garbutt JS, Belles X, Richards EH, Reynolds SE, 2013. Persistence of double-stranded RNA in insect hemolymph as a potential determiner of RNA interference success: Evidence from Manduca sexta and Blattella germanica. Journal of Insect Physiology 59, 171-8.

Geldhof $P$, Murray L, Couthier A, et al., 2006. Testing the efficacy of RNA interference in Haemonchus contortus. International Journal for Parasitology 36, 801-10.

Ghedin E, Hailemariam T, Depasse JV, et al., 2009. Brugia malayi Gene Expression in Response to the Targeting of the Wolbachia Endosymbiont by Tetracycline Treatment. Plos Neglected Tropical Diseases 3.

Gibbings D, Voinnet $\mathrm{O}, 2010$. Control of RNA silencing and localization by endolysosomes. Trends in Cell Biology 20, 491-501. 
Giordano E, Rendina R, Peluso I, Furia M, 2002. RNAi triggered by symmetrically transcribed transgenes in Drosophila melanogaster. Genetics 160, 637-48.

Goto A, Blandin S, Royet J, Reichhart JM, Levashina EA, 2003. Silencing of Toll pathway components by direct injection of double-stranded RNA into Drosophila adult flies. Nucleic Acids Research 31, 6619-23.

Grainger JR, Smith KA, Hewitson JP, et al., 2010. Helminth secretions induce de novo T cell Foxp3 expression and regulatory function through the TGF- $\beta$ pathway. Journal of Experimental Medicine 207, 2331-41.

Grbić M, Van Leeuwen T, Clark RM, et al., 2011. The genome of Tetranychus urticae reveals herbivorous pest adaptations. Nature 479, 487-92.

Grishok A, Tabara H, Mello CC, 2000. Genetic requirements for inheritance of RNAi in C-elegans. Science 287, 2494-7.

Gu L, Knipple DC, 2013. Recent advances in RNA interference research in insects: Implications for future insect pest management strategies. Crop Protection 45, 36-40.

Hardy JL, Houk EJ, Kramer LD, Reeves WC, 1983. Intrinsic factors affecting vector competence of mosquitoes for arboviruses. Annual Review of Entomology 28, 229-62.

Hinas A, Wright AJ, Hunter CP, 2012. SID-5 Is an Endosome-Associated Protein Required for Efficient Systemic RNAi in C. elegans. Current Biology 22, 1938-43.

Hoogstrate SW, Volkers RJ, Sterken MG, Kammenga JE, Snoek LB, 2014. Nematode endogenous small RNA pathways. Worm 3, e28234-e.

Huang G, Allen R, Davis EL, Baum TJ, Hussey RS, 2006. Engineering broad root-knot resistance in transgenic plants by RNAi silencing of a conserved and essential root-knot nematode parasitism gene. Proceedings of the National Academy of Sciences of the United States of America 103, 14302-6.

Huang J-H, Jing $X$, Douglas $A E, 2015$. The multi-tasking gut epithelium of insects. Insect Biochemistry and Molecular Biology 67, 15-20.

Hunter CP, Winston WM, Molodowitch C, et al., 2006. Systemic RNAi in Caenorhabditis elegans. Cold Spring Harbor symposia on quantitative biology 71, 95-100.

Hussein AS, Kichenin K, Selkirk ME, 2002. Suppression of secreted acetylcholinesterase expression in Nippostrongylus brasiliensis by RNA interference. Molecular and Biochemical Parasitology 122, 914.

Ibrahim HMM, Alkharouf NW, Meyer SLF, et al., 2011. Post-transcriptional gene silencing of root-knot nematode in transformed soybean roots. Experimental parasitology 127, 90-9.

Imae R, Dejima K, Kage-Nakadai E, Arai H, Mitani S, 2016. Endomembrane-associated RSD-3 is important for RNAi induced by extracellular silencing RNA in both somatic and germ cells of Caenorhabditis elegans. Scientific Reports 6.

Issa Z, Grant W, Stasiuk S, Shoemaker C, 2005. Development of methods for RNA interference in the sheep gastrointestinal parasite, Trichostrongylus colubriformis. International Journal for Parasitology 35, 935-40.

Jarosch A, Moritz RFA, 2011. Systemic RNA-interference in the honeybee Apis mellifera: Tissue dependent uptake of fluorescent siRNA after intra-abdominal application observed by laserscanning microscopy. Journal of Insect Physiology 57, 851-7.

Jeffers LA, Roe RM, 2008. The movement of proteins across the insect and tick digestive system. Journal of Insect Physiology 54, 319-32. 
Jose AM, 2015. Movement of regulatory RNA between animal cells. Genesis 53, 395-416.

Jose AM, Hunter CP, 2007. Transport of sequence-specific RNA interference information between cells. In. Annual Review of Genetics. 305-30. (Annual Review of Genetics; vol. 41.)

Jose AM, Kim YA, Leal-Ekman S, Hunter CP, 2012. Conserved tyrosine kinase promotes the import of silencing RNA into Caenorhabditis elegans cells. Proceedings of the National Academy of Sciences of the United States of America 109, 14520-5.

Jose AM, Smith JJ, Hunter CP, 2009. Export of RNA silencing from C-elegans tissues does not require the RNA channel SID-1. Proceedings of the National Academy of Sciences of the United States of America 106, 2283-8.

Kalidas S, Smith DP, 2002. Novel genomic cDNA hybrids produce effective RNA interference in adult Drosophila. Neuron 33, 177-84.

Khajuria C, Velez AM, Rangasamy M, et al., 2015. Parental RNA interference of genes involved in embryonic development of the western corn rootworm, Diabrotica virgifera virgifera LeConte. Insect Biochemistry and Molecular Biology 63, 54-62.

Khila A, Grbic M, 2007. Gene silencing in the spider mite Tetranychus urticae: dsRNA and siRNA parental silencing of the Distal-less gene. Development Genes and Evolution 217, 241-51.

Killiny N, Hajeri S, Tiwari S, Gowda S, Stelinski LL, 2014. Double-Stranded RNA Uptake through Topical Application, Mediates Silencing of Five CYP4 Genes and Suppresses Insecticide Resistance in Diaphorina citri. PLOS ONE 9.

Kimber MJ, Mckinney S, Mcmaster S, Day TA, Fleming CC, Maule AG, 2007. flp gene disruption in a parasitic nematode reveals motor dysfunction and unusual neuronal sensitivity to RNA interference. Faseb Journal 21, 1233-43.

Klink VP, Kim K-H, Martins V, et al., 2009. A correlation between host-mediated expression of parasite genes as tandem inverted repeats and abrogation of development of female Heterodera glycines cyst formation during infection of Glycine max. Planta 230, 53-71.

Knight M, Miller A, Liu Y, Scaria P, Woodle M, Ittiprasert W, 2011. Polyethyleneimine (PEI) Mediated siRNA Gene Silencing in the Schistosoma mansoni Snail Host, Biomphalaria glabrata. Plos Neglected Tropical Diseases 5.

Kocks C, Cho JH, Nehme N, et al., 2005. Eater, a transmembrane protein mediating phagocytosis of bacterial pathogens in Drosophila. Cell 123, 335-46.

Konopova B, Akam M, 2014. The Hox genes Ultrabithorax and abdominal-A specify three different types of abdominal appendage in the springtail Orchesella cincta (Collembola). Evodevo 5.

Krautz-Peterson G, Bhardwaj R, Faghiri Z, Tararam CA, Skelly PJ, 2010. RNA interference in schistosomes: machinery and methodology. Parasitology 137, 485-95.

Kroeger PT, Jr., Tokusumi T, Schulz RA, 2012. Transcriptional regulation of eater gene expression in Drosophila blood cells. Genesis 50, 41-9.

Kurscheid S, Lew-Tabor AE, Valle MR, et al., 2009. Evidence of a tick RNAi pathway by comparative genomics and reverse genetics screen of targets with known loss-of-function phenotypes in Drosophila. Bmc Molecular Biology 10, 26.

Kyaw Min A, Boldbaatar D, Umemiya-Shirafuji R, et al., 2011. Scavenger receptor mediates systemic RNA interference in ticks. PLoS ONE, e28407.

Lau NC, Lim LP, Weinstein EG, Bartel DP, 2001. An abundant class of tiny RNAs with probable regulatory roles in Caenorhabditis elegans. Science 294, 858-62.

The present document has been produced and adopted by the bodies identified above as authors. This task has been carried out exclusively by the author(s) in the context of a contract between the European Food Safety Authority and the authors, awarded following a tender procedure. The present document is published complying with the transparency principle to which the Authority is subject. It may not be considered as an output adopted by the Authority. The European Food Safety Authority reserves its rights, view and position as regards the issues addressed and the conclusions reached in the present document, without prejudice to the rights of the authors. 
Lee YS, Pressman S, Andress AP, et al., 2009. Silencing by small RNAs is linked to endosomal trafficking. Nature Cell Biology 11, 1150-U243.

Legendre-Guillemin V, Wasiak S, Hussain NK, Angers A, Mcpherson PS, 2004. ENTH/ANTH proteins and clathrin-mediated membrane budding. Journal of Cell Science 117, 9-18.

Li H, Bowling AJ, Gandra P, et al., 2016. Systemic RNAi in western corn rootworm, Diabrotica virgifera virgifera, does not involve transitive pathways. Insect Science.

Li J, Todd TC, Oakley TR, Lee J, Trick HN, 2010. Host-derived suppression of nematode reproductive and fitness genes decreases fecundity of Heterodera glycines Ichinohe. Planta 232, 775-85.

Li X, Dong X, Zou C, Zhang H, 2015. Endocytic pathway mediates refractoriness of insect Bactrocera dorsalis to RNA interference. Scientific Reports 5.

Li X, Zhang M, Zhang H, 2011. RNA Interference of Four Genes in Adult Bactrocera dorsalis by Feeding Their dsRNAs. PLOS ONE 6.

Lilley $\mathrm{CJ}$, Davies $\mathrm{L}$, Urwin PE, 2012. RNA interference in plant parasitic nematodes: a summary of the current status. Parasitology 139, 630-40.

Liu $\mathrm{H}$, Wang $\mathrm{X}$, Wang $\mathrm{H}$, et al., 2012a. Escherichia coli noncoding RNAs can affect gene expression and physiology of Caenorhabditis elegans. Nature Communications 3, 1073.

Liu J, Swevers L, Iatrou K, Huvenne H, Smagghe G, 2012b. Bombyx mori DNA/RNA non-specific nuclease: Expression of isoforms in insect culture cells, subcellular localization and functional assays. Journal of Insect Physiology 58, 1166-76.

Liu PZ, Kaufman TC, 2004a. hunchback is required for suppression of abdominal identity, and for proper germband growth and segmentation in the intermediate germband insect Oncopeltus fasciatus. Development (Cambridge) 131, 1515-27.

Liu PZ, Kaufman TC, 2004b. Kruppel is a gap gene in the intermediate germband insect Oncopeltus fasciatus and is required for development of both blastoderm and germ band-derived segments. Development 131, 4567-79.

Louvet-Vallée S, Kolotuev I, Podbilewicz B, Félix M-A, 2003. Control of vulval competence and centering in the nematode Oscheius sp. 1 CEW1. Genetics 163, 133-46.

Luo Y, Wang X, Wang X, Yu D, Chen B, Kang L, 2013. Differential responses of migratory locusts to systemic RNA interference via double-stranded RNA injection and feeding. Insect Molecular Biology 22, 574-83.

Luo Y, Wang X, Yu D, Kang L, 2012. The SID-1 double-stranded RNA transporter is not required for systemic RNAi in the migratory locust. RNA biology 9, 663-71.

Lynch JA, Desplan C, 2006. A method for parental RNA interference in the wasp Nasonia vitripennis. Nature Protocols 1, 486-94.

Maizels RM, Hewitson JP, Murray J, et al., 2012. Immune modulation and modulators in Heligmosomoides polygyrus infection. Experimental parasitology 132, 76-89.

Mao J, Liu C, Zeng F, 2013. Hunchback is required for abdominal identity suppression and germband growth in the parthenogenetic embryogenesis of the pea aphid, Acyrthosiphon pisum. Archives of Insect Biochemistry and Physiology 84, 209-21.

Mcewan DL, Weisman AS, Hunter CP, 2012. Uptake of extracellular double-stranded RNA by SID-2. Molecular Cell 47, 746-54.

Meng L, Chen L, Li Z, Wu Z, Shan G, 2013. Environmental RNA interference in animals. Chinese Science Bulletin 58, 4418-25. 
Miller SC, Miyata K, Brown SJ, Tomoyasu Y, 2012. Dissecting Systemic RNA Interference in the Red Flour Beetle Tribolium castaneum: Parameters Affecting the Efficiency of RNAi. PLoS ONE 7.

Mito T, Okamoto $H$, Shinahara $W$, et al., 2006. Kruppel acts as a gap gene regulating expression of hunchback and even-skipped in the intermediate germ cricket Gryllus bimaculatus. Developmental Biology 294, 471-81.

Mito T, Ronco M, Uda T, Nakamura T, Ohuchi H, Noji S, 2008. Divergent and conserved roles of extradenticle in body segmentation and appendage formation, respectively, in the cricket Gryllus bimaculatus. Developmental Biology 313, 67-79.

Miyata K, Brown SJ, Miller SC, Tomoyasu Y, 2012. Dissecting Systemic RNA Interference in the Red Flour Beetle Tribolium castaneum: Parameters Affecting the Efficiency of RNAi. Figshare 1.

Miyata K, Ramaseshadri P, Zhang Y, Segers G, Bolognesi R, Tomoyasu Y, 2014. Establishing an In Vivo Assay System to Identify Components Involved in Environmental RNA Interference in the Western Corn Rootworm. PLOS ONE 9.

Paim RMM, Araujo RN, Lehane MJ, Gontijo NF, Pereira MH, 2013. Long-term effects and parental RNAi in the blood feeder Rhodnius prolixus (Hemiptera; Reduviidae). Insect Biochemistry and Molecular Biology 43, 1015-20.

Pak J, Fire A, 2007. Distinct populations of primary and secondary effectors during RNAi in C. elegans. Science (Washington) 315, 241-4.

Parrish S, Fleenor J, Xu SQ, Mello C, Fire A, 2000. Functional anatomy of a dsRNA trigger: Differential requirement for the two trigger strands in RNA interference. Molecular Cell 6, 1077-87.

Parry $\mathrm{DH}, \mathrm{Xu} \mathrm{JL}$, Ruvkun G, 2007. A whole-genome RNAi screen for C. elegans miRNA pathway genes. Current Biology 17, 2013-22.

Patel N, Hamamouch N, Li C, et al., 2010. A nematode effector protein similar to annexins in host plants. Journal of Experimental Botany 61, 235-48.

Patel N, Hamamouch N, Li C, et al., 2008. Similarity and Functional Analyses of Expressed Parasitism Genes in Heterodera schachtii and Heterodera glycines. Journal of Nematology 40, 299-310.

Pearson A, Lux A, Krieger M, 1995. Expression cloning of dSR-CI, a class C macrophage-specific scavenger receptor from Drosophila melanogaster. Proceedings of the National Academy of Sciences 92, 4056-60.

Pires-Dasilva A, Sommer RJ, 2004. Conservation of the global sex determination gene tra-1 in distantly related nematodes. Genes \& Development 18, 1198-208.

Pitino M, Coleman AD, Maffei ME, Ridout CJ, Hogenhout SA, 2011. Silencing of Aphid Genes by dsRNA Feeding from Plants. PLOS ONE 6.

Piulachs MD, Pagone $V$, Bellés $X, 2010$. Key roles of the Broad-Complex gene in insect embryogenesis. Insect Biochemistry and Molecular Biology 40, 468-75.

Powell ME, Bradish HM, Gatehouse JA, Fitches EC, 2017. Systemic RNAi in the small hive beetle Aethina tumida Murray (Coleoptera: Nitidulidae), a serious pest of the European honey bee Apis mellifera. Pest Management Science 73, 53-63.

Pridgeon JW, Zhao L, Becnel JJ, Strickman DA, Clark GG, Linthicum KJ, 2008. Topically applied AaeIAP1 double-stranded RNA kills female adults of Aedes aegypti. Journal of Medical Entomology 45, 414-20.

Quintana JF, Babayan SA, Buck AH, 2016. Small RNAs and extracellular vesicles in filarial nematodes: from nematode development to diagnostics. Parasite Immunology.

The present document has been produced and adopted by the bodies identified above as authors. This task has been carried out exclusively by the author(s) in the context of a contract between the European Food Safety Authority and the authors, awarded following a tender procedure. The present document is published complying with the transparency principle to which the Authority is subject. It may not be considered as an output adopted by the Authority. The European Food Safety Authority reserves its rights, view and position as regards the issues addressed and the conclusions reached in the present document, without prejudice to the rights of the authors. 
Rämet M, Pearson A, Manfruelli $P$, et al., 2001. Drosophila scavenger receptor $C I$ is a pattern recognition receptor for bacteria. Immunity 15, 1027-38.

Rocheleau CE, 2012. RNA Interference: Systemic RNAi SIDes with Endosomes. Current Biology 22, R873-R5.

Roignant JY, Carre C, Mugat R, Szymczak D, Lepesant JA, Antoniewski C, 2003. Absence of transitive and systemic pathways allows cell-specific and isoform-specific RNAi in Drosophila. Rna-a Publication of the Rna Society 9, 299-308.

Ronco M, Uda T, Mito T, Minelli A, Noji S, Klingler M, 2008. Antenna and all gnathal appendages are similarly transformed by homothorax knock-down in the cricket Gryllus bimaculatus. Developmental Biology 313, 80-92.

Rosso MN, Dubrana MP, Cimbolini N, Jaubert S, Abad P, 2005. Application of RNA interference to root-knot nematode genes encoding esophageal gland proteins. Molecular Plant-Microbe Interactions $18,615-20$.

Saleh M-C, Tassetto M, Van Rij RP, et al., 2009. Antiviral immunity in Drosophila requires systemic RNA interference spread. Nature 458, 346-U109.

Saleh M-C, Van Rij RP, Hekele A, et al., 2006a. dsRNA uptake and processing. GenomeRNAi.

Saleh M-C, Van Rij RP, Hekele A, et al., 2006b. The endocytic pathway mediates cell entry of dsRNA to induce RNAi silencing. Nature Cell Biology 8, 793-U19.

Santos D, Broeck JV, Wynant N, 2014. Systemic RNA interference in locusts: reverse genetics and possibilities for locust pest control. Current Opinion in Insect Science 6, 9-14.

Sarkies P, Miska EA, 2014. Small RNAs break out: the molecular cell biology of mobile small RNAs. Nature Reviews Molecular Cell Biology 15, 525-35.

Schumpert CA, Dudycha JL, Patel RC, 2015. Development of an efficient RNA interference method by feeding for the microcrustacean Daphnia. Bmc Biotechnology 15.

Shannon AJ, Tyson T, Dix I, Boyd J, Burnell AM, 2008. Systemic RNAi mediated gene silencing in the anhydrobiotic nematode Panagrolaimus superbus. Bmc Molecular Biology 9.

Shih JD, Fitzgerald MC, Sutherlin M, Hunter CP, 2009. The SID-1 double-stranded RNA transporter is not selective for dsRNA length. Rna-a Publication of the Rna Society 15, 384-90.

Shih JD, Hunter CP, 2011. SID-1 is a dsRNA-selective dsRNA-gated channel. Rna-a Publication of the Rna Society 17, 1057-65.

Sijen T, Fleenor J, Simmer F, et al., 2001. On the role of RNA amplification in dsRNA-triggered gene silencing. Cell 107, 465-76.

Sijen T, Steiner FA, Thijssen KL, Plasterk RHA, 2007. Secondary siRNAs result from unprimed RNA synthesis and form a distinct class. Science 315, 244-7.

Simmer F, Tijsterman M, Parrish S, et al., 2002. Loss of the putative RNA-directed RNA polymerase RRF-3 makes C. elegans hypersensitive to RNAi. Current Biology 12, 1317-9.

Sindhu AS, Maier TR, Mitchum MG, Hussey RS, Davis EL, Baum TJ, 2009. Effective and specific in planta RNAi in cyst nematodes: expression interference of four parasitism genes reduces parasitic success. Journal of Experimental Botany 60, 315-24.

Smardon A, Spoerke JM, Stacey SC, Klein ME, Mackin N, Maine EM, 2000. EGO-1 is related to RNAdirected RNA polymerase and functions in germ-line development and RNA interference in Celegans. Current Biology 10, 169-78.

The present document has been produced and adopted by the bodies identified above as authors. This task has been carried out exclusively by the author(s) in the context of a contract between the European Food Safety Authority and the authors, awarded following a tender procedure. The present document is published complying with the transparency principle to which the Authority is subject. It may not be considered as an output adopted by the Authority. The European Food Safety Authority reserves its rights, view and position as regards the issues addressed and the conclusions reached in the present document, without prejudice to the rights of the authors. 
Soares CaG, Lima CMR, Dolan MC, Piesman J, Beard CB, Zeidner NS, 2005. Capillary feeding of specific dsRNA induces silencing of the isac gene in nymphal Ixodes scapularis ticks. Insect Molecular Biology 14, 443-52.

Song C, Gallup JM, Day TA, Bartholomay LC, Kimber MJ, 2010. Development of an in vivo RNAi protocol to investigate gene function in the filarial nematode, Brugia malayi. PLoS Pathog 6, e1001239.

Steeves RM, Todd TC, Essig JS, Trick HN, 2006. Transgenic soybeans expressing siRNAs specific to a major sperm protein gene suppress Heterodera glycines reproduction. Functional Plant Biology 33, 991-9.

Sundaram P, Echalier B, Han W, Hull D, Timmons L, 2006. ATP-binding cassette transporters are required for efficient RNA interference in Caenorhabditis elegans. Molecular Biology of the Cell 17, 3678-88.

Tabara H, Grishok A, Mello CC, 1998. RNAi in C. elegans: Soaking in the genome sequence. Science (Washington) 282, 430-1.

Taning CNT, Christiaens O, Berkvens N, Casteels H, Maes M, Smagghe G, 2016. Oral RNAi to control Drosophila suzukii: laboratory testing against larval and adult stages. Journal of Pest Science 89, 803-14.

Teo M, Tan L, Lim L, Manser E, 2001. The tyrosine kinase ACK1 associates with clathrin-coated vesicles through a binding motif shared by arrestin and other adaptors. Journal of Biological Chemistry 276, 18392-8.

Terenius O, Papanicolaou A, Garbutt JS, et al., 2011. RNA interference in Lepidoptera: An overview of successful and unsuccessful studies and implications for experimental design. Journal of Insect Physiology 57, 231-45.

Tian H, Tang B, Xie Q, et al., 2009. Developmental Control of a Lepidopteran Pest Spodoptera exigua by Ingestion of Bacteria Expressing dsRNA of a Non-Midgut Gene. Figshare.

Tijsterman M, May RC, Simmer F, Okihara KL, Plasterk RHA, 2004. Genes required for systemic RNA interference in Caenorhabditis elegans. Current Biology 14, 111-6.

Timmons L, Court DL, Fire A, 2001. Ingestion of bacterially expressed dsRNAs can produce specific and potent genetic interference in Caenorhabditis elegans. Gene 263, 103-12.

Timmons L, Fire A, 1998. Specific interference by ingested dsRNA. Nature (London) 395, 854.

Timmons L, Tabara $\mathrm{H}$, Mello CC, Fire AZ, 2003. Inducible systemic RNA silencing in Caenorhabditis elegans. Molecular Biology of the Cell 14, 2972-83.

Tomoyasu Y, Miller SC, Tomita S, Schoppmeier M, Grossmann D, Bucher G, 2008. Exploring systemic RNA interference in insects: a genome-wide survey for RNAi genes in Tribolium. Genome Biology 9.

Tsai H-Y, Chen C-CG, Conte D, Jr., et al., 2015. A Ribonuclease Coordinates siRNA Amplification and mRNA Cleavage during RNAi. Cell 160, 407-19.

Turner CT, Davy MW, Macdiarmid RM, Plummer KM, Birch NP, Newcomb RD, 2006. RNA interference in the light brown apple moth, Epiphyas postvittana (Walker) induced by double-stranded RNA feeding. Insect Molecular Biology 15, 383-91.

Ulvila J, Parikka M, Kleino A, et al., 2006. Double-stranded RNA is internalized by scavenger receptormediated endocytosis in Drosophila S2 cells. Journal of Biological Chemistry 281, 14370-5.

Urwin PE, Lilley $\mathrm{C}$, Atkinson $\mathrm{HJ}$, 2002. Ingestion of double-stranded RNA by preparasitic juvenile cyst nematodes leads to RNA interference. Molecular Plant-Microbe Interactions 15, 747-52.

The present document has been produced and adopted by the bodies identified above as authors. This task has been carried out exclusively by the author(s) in the context of a contract between the European Food Safety Authority and the authors, awarded following a tender procedure. The present document is published complying with the transparency principle to which the Authority is subject. It may not be considered as an output adopted by the Authority. The European Food Safety Authority reserves its rights, view and position as regards the issues addressed and the conclusions reached in the present document, without prejudice to the rights of the authors. 
Vastenhouw NL, Brunschwig K, Okihara KL, Mueller F, Tijsterman M, Plasterk RHA, 2006. Long-term gene silencing by RNAi. Nature 442, 882-.

Vélez AM, Fishilevich E, Matz N, Storer NP, Narva KE, Siegfried BD, 2016. Parameters for Successful Parental RNAi as An Insect Pest Management Tool in Western Corn Rootworm, Diabrotica virgifera virgifera. Genes 8, 7.

Viney ME, Thompson FJ, 2008. Two hypotheses to explain why RNA interference does not work in animal parasitic nematodes. International Journal for Parasitology 38, 43-7.

Visser A, Geldhof P, De Maere V, Knox D, Vercruysse J, Claerebout E, 2006. Efficacy and specificity of RNA interference in larval life-stages of Ostertagia ostertagi. Parasitology 133, 777.

Wang G, Li N, Zhang L, Zhang L, Zhang Z, Wang Y, 2016. IGFBP7 is involved in abalone metamorphosis. Aquaculture 451, 377-84.

Wang Y, Li H, Miao X, Zhang H, 2011. Second-Generation Sequencing Supply an Effective Way to Screen RNAi Targets in Large Scale for Potential Application in Pest Insect Control. Figshare.

Waqas A, Shan G, 2016. Uptake and Reaction of C. elegans to Environmental RNAs. In. Non-coding RNAs and Inter-kingdom Communication. Springer, 117-24.

Whangbo JS, Hunter CP, 2008. Environmental RNA interference. Trends in Genetics 24, 297-305.

Wheeler D, Darby BJ, Todd TC, Herman MA, 2012. Several Grassland Soil Nematode Species Are Insensitive to RNA-Mediated Interference. Journal of Nematology 44, 92-101.

Whyard S, Singh AD, Wong $S, 2009$. Ingested double-stranded RNAs can act as species-specific insecticides. Insect Biochemistry and Molecular Biology 39, 824-32.

Winston WM, Molodowitch C, Hunter CP, 2002. Systemic RNAi in C-elegans requires the putative transmembrane protein SID-1. Science 295, 2456-9.

Winston WM, Sutherlin M, Wright AJ, Feinberg EH, Hunter CP, 2007. Caenorhabditis elegans SID-2 is required for environmental RNA interference. Proceedings of the National Academy of Sciences of the United States of America 104, 10565-70.

Worby CA, Simonson-Leff N, Dixon JE, 2001. RNA interference of gene expression (RNAi) in cultured Drosophila cells. Sci Stke 95, PL1.

Wu K, Hoy MA, 2014. Oral delivery of double-stranded RNA induces prolonged and systemic gene knockdown in Metaseiulus occidentalis only after feeding on Tetranychus urticae. Experimental and Applied Acarology 63, 171-87.

Wynant N, Santos D, Van Wielendaele P, Broeck JV, 2014a. Scavenger receptor-mediated endocytosis facilitates RNA interference in the desert locust, Schistocerca gregaria. Insect Molecular Biology 23, 320-9.

Wynant N, Santos D, Verdonck R, Spit J, Van Wielendaele P, Vanden Broeck J, 2014b. Identification, functional characterization and phylogenetic analysis of double stranded RNA degrading enzymes present in the gut of the desert locust, Schistocerca gregaria. Insect Biochemistry and Molecular Biology 46, 1-8.

Wynant N, Verlinden H, Breugelmans B, Simonet G, Broeck JV, 2012. Tissue-dependence and sensitivity of the systemic RNA interference response in the desert locust, Schistocerca gregaria. Insect Biochemistry and Molecular Biology 42, 911-7.

Xiao D, Gao X, Xu J, et al., 2015. Clathrin-dependent endocytosis plays a predominant role in cellular uptake of double-stranded RNA in the red flour beetle. Insect Biochemistry and Molecular Biology $60,68-77$. procedure. The present document is published complying with the transparency principle to which the Authority is subject. It may not be considered as an output adopted by the Authority. The European Food Safety Authority reserves its rights, view and position as regards the issues addressed and the conclusions reached in the present document, without prejudice to the rights of the authors. 
Xu HJ, Chen T, Ma XF, et al., 2013. Genome-wide screening for components of small interfering RNA (siRNA) and micro-RNA (miRNA) pathways in the brown planthopper, Nilaparvata lugens (Hemiptera: Delphacidae). Insect Molecular Biology 22, 635-47.

Yang W, Lo CG, Dispenza T, Cerione RA, 2001. The Cdc42 target ACK2 directly interacts with clathrin and influences clathrin assembly. Journal of Biological Chemistry 276, 17468-73.

Yoshiyama N, Tojo K, Hatakeyama M, 2013. A survey of the effectiveness of non-cell autonomous RNAi throughout development in the sawfly, Athalia rosae (Hymenoptera). Journal of Insect Physiology 59, 400-7.

Zhang C, Montgomery TA, Fischer SE, et al., 2012. The Caenorhabditis elegans RDE-10/RDE-11 complex regulates RNAi by promoting secondary siRNA amplification. ArrayExpress Archive.

Zhang H, Li H, Guan R, Miao X, 2015. Lepidopteran insect species-specific, broad-spectrum, and systemic RNA interference by spraying dsRNA on larvae. Entomologia Experimentalis Et Applicata $155,218-28$.

Zheng G, Ambros V, Li W-H, 2010. Inhibiting miRNA in Caenorhabditis elegans using a potent and selective antisense reagent. Silence 1, 9-.

Zhu F, Xu J, Palli R, Ferguson J, Palli SR, 2011. Ingested RNA interference for managing the populations of the Colorado potato beetle, Leptinotarsa decemlineata. Pest Management Science $67,175-82$.

The present document has been produced and adopted by the bodies identified above as authors. This task has been carried out exclusively by the author(s) in the context of a contract between the European Food Safety Authority and the authors, awarded following a tender procedure. The present document is published complying with the transparency principle to which the Authority is subject. It may not be considered as an output adopted by the Authority. The European Food Safety Authority reserves its rights, view and position as regards the issues addressed and the conclusions reached in the present document, without prejudice to the rights of the authors. 


\subsection{Environmental exposure and fate of dsRNA, siRNA and miRNA (Task 3)}

\subsubsection{Introduction}

In this section, we consider studies on environmental exposure to and fate of dsRNA, siRNA and miRNA and the factors affecting exposure levels. We first focus on environmental exposure routes from plants to invertebrates in general, drawing on what is known from other plant biotechnologies. In a second part, we present the literature specifically dealing with dsRNA molecules in this context, such as expression levels in GM plants and stability of dsRNA in the environment. Furthermore, potential barriers in invertebrates are also considered, but are discussed more in detail in Section 5.3 which deals with efficiency of RNAi in invertebrates.

\subsubsection{Environmental exposure routes from plants to invertebrates}

Plants are the primary producers in both grazing and detritus food chains linked to cropping systems. Plant tissue-expressed compounds can enter food chains through primary and secondary routes, and a number of invertebrate organisms at different trophic levels can be exposed to them. A schematic representation of the possible routes of exposure discussed above is given in Table 9.

\subsubsection{Direct exposure through plant consumption}

The primary route of exposure from plants to invertebrates is through direct feeding on plant living material (herbivory) above or below ground, or through plant residues in the case of detritivores.

Many primary consumers are usually linked to plants as their food source. The variety of feeding habits is quite large and, basically, all plant parts can be ingested by herbivorous invertebrates, including leaves, seeds, pollen, phloem sap, guttation, nectar, roots, tubers/corms. Initial exposure of a herbivore, and possibly the higher trophic species feeding on the herbivore, will depend on expression levels in the plant tissues consumed by the herbivore. In the case of some GM plants, the newly expressed trait is sometimes not expressed or expressed at low levels in certain tissues such as pollen, nectar or phloem. Hence, species feeding exclusively on these tissues may not be exposed or affected. It is therefore important to determine which plant tissues are expressing RNAi in order to determine exposure levels in invertebrate herbivores with different feeding habits.

Cultivated plants represent the first point of exposure for herbivores. However, during or after the growing season, plant tissues may be dispersed in the form of pollen and seeds, or, occasionally, as plant residues on the soil surface. Dispersal occurs at variable distances, due to air, water and soil movement, and by the activity of animals. Therefore, exposure can occur remotely from source plants, and may be significant in relation to pollen collected by insects and harvested seeds, roots, tubers and corms transported to storage and processing facilities.

Seed dispersal of most cultivated species normally occurs over short distances, though there are some exceptions: for example, some fruit crop seeds can be dispersed in animal faeces (Cain et al., 2000), and seeds of some grasses can be widely dispersed by wind, e.g., GM creeping bent grass (Zapiola et al., 2008). Insects and wind can be efficient means of dispersing pollen and the extent of pollen dispersal depends on the crop species, flower and pollen morphology, the vectors and the local meteorological conditions. Several studies on crops such as maize indicate that the large majority of wind-dispersed pollen grains were deposited at relatively short distances from the source (Lavigne et al., 1998; Treu et al., 2000). Pollen grains of other crop species may move over significantly larger distances, with bees foraging over distances of several kilometers (McCartney and Lacey, 1991).

Pollen and nectar feeders include bees, wasps, pollen beetles, etc., but invaders of nests and hives (e.g., wax moth, ants) could also be exposed to it. Foraging adult insects (e.g., bees) will transport pollen and nectar to larvae so that they are also exposed. Exposure to pollen may also occur through

The present document has been produced and adopted by the bodies identified above as authors. This task has been carried out exclusively by the author(s) in the context of a contract between the European Food Safety Authority and the authors, awarded following a tender procedure. The present document is published complying with the transparency principle to which the Authority is subject. It may not be considered as an output adopted by the Authority. The European Food Safety Authority reserves its rights, view and position as regards the issues addressed and the conclusions reached in the present document, without prejudice to the rights of the authors. 
other routes, e.g., maize pollen transported by wind can be deposited on the leaves of food plants of non-target species in the vicinity of cultivation areas. If the pollen is derived from GM plants expressing insecticidal traits (e.g., Cry proteins), the non target species may become exposed to it when feeding on the leaves of their food plants (Felke et al., 2010; Gathmann et al., 2006).

The data collected on the extent of pollen deposition on wild plants are quite variable (e.g., Gathmann et al., 2006; Hofmann et al., 2016; Lang et al., 2004). The actual exposure to GM pollen for a given non-target species will ultimately depend on the presence of individuals of the species during pollen shed in the area, the availability of their host plants, their distance from pollen source and the pollen concentration at the emission source. The combination of all these factors in the specific receiving environment can be very different even for the same crop species. For instance, the estimate of the potential exposure of non-target butterfly larvae to maize pollen in different growing regions, may produce substantially different results (Arpaia et al., 2018).

The possible exposure of NTOs to plants is not limited to the growing season. After harvest, plant residues and most of the root system remain in the soil until degradation. Depending upon the harvesting practices and the cultivated species, part of the residues might also be incorporated into the soil for enriching the reservoir of organic matter. Plant residues during and after mechanical harvesting may be transported outside cropped fields and enter soil and aquatic systems where it could remain near the site of entry or be further dispersed by water currents (e.g., Rosi-Marshall et al., 2007; Poté et al., 2009; Tank et al., 2010).

The fate of GM plant residues in soil has been previously studied. For instance, Cry proteins are known to bind to clay, suggesting that there is potential for their long term persistence and consequently long exposure to NTOs (Icoz and Stotzky, 2008). Degradation of plant tissue will affect the potential for exposure to nucleic acids, since DNA is degraded within the plant during senescence (Pietramellara et al., 2009). However, a certain amount of dsRNA could be expected to be released in the soil.

Depending on the crop species considered, possible outcrossing to wild/weedy relatives may occur. Consequently, the above exposure routes may be applicable to the progeny of the outcrossed plants expressing traits passed from a transformed crop. The potential for outcrossing and introgression of traits from crops to wild relatives has been extensively reviewed, (e.g., Eastham and Sweet, 2002; Ellstrand et al., 2013).

\subsubsection{Indirect exposure through the food chain}

Secondary exposure can occur through indirect feeding (exposure through multi-trophic interactions) e.g., natural enemies feeding on herbivores. Food webs in agro-ecosystems are typically simplified compared to natural habitats due to the major impact of human activities, the short time span for which plants remain in the field and the usual uniformity of cultivated plants. Even so, rather complex multi-trophic relationships are established between primary producers and consumers, at least up to the fourth trophic level (Verkerk, 2004). A herbivore represents a possible host/prey of one or more species of natural enemies. Upon ingestion, a plant compound can be present in the body of a herbivore as such, or as one of its metabolites. When predators or parasitoids feed on herbivores, they are consequently exposed to the plant-derived product and/or its metabolites. 
Table 9: Possible channels and routes of exposure of non-target organisms to plant-expressed compounds

\begin{tabular}{llll}
\hline Channel & Mechanism & Exposed invertebrates & Site of exposure \\
\hline Air & Pollen flow, seed dispersal & $\begin{array}{l}\text { Herbivores ingesting pollen, } \\
\text { seeds }\end{array}$ & $\begin{array}{l}\text { Plants, soils and water } \\
\text { bodies inside and outside } \\
\text { cultivated areas }\end{array}$ \\
Plant & Ingestion of plant parts & $\begin{array}{l}\text { Primary consumers } \\
\text { (herbivores, including } \\
\text { pollinators); } \\
\text { Higher order consumers } \\
\text { (predators, parasitoids, } \\
\text { etc.) }\end{array}$ & $\begin{array}{l}\text { Cultivated areas, plants } \\
\text { and plant propagules/parts } \\
\text { removed from cultivated } \\
\text { areas and sites of } \\
\text { wild/weedy relatives of the } \\
\text { crop }\end{array}$ \\
$\begin{array}{l}\text { Soil } \\
\text { (including soil } \\
\text { water) }\end{array}$ & $\begin{array}{l}\text { Release of plant products } \\
\text { (e.g., exudates), } \\
\text { Plant residues in the soil } \\
\text { and soil water }\end{array}$ & $\begin{array}{l}\text { Meso- and macro-fauna } \\
\text { (detritivores, higher order } \\
\text { consumers), aquatic } \\
\text { species }\end{array}$ & $\begin{array}{l}\text { Cultivated field, drainage } \\
\text { systems, water courses }\end{array}$ \\
\hline
\end{tabular}

Predators usually consume several individuals of their preys when feeding, and therefore accumulation of plant products may also occur at higher trophic levels. Svobodová et al. (2017) found that, among different predators, ladybeetle larvae showed higher concentrations than lacewing larvae and juvenile spiders. A particular case of tritrophic relationships occurs in nature with some higher order consumers, feeding on products made by herbivores (e.g., aphids' honeydew). For instance, ants are well known for obtaining a large portion of their carbohydrate needs by collecting the sugarrich honeydew of aphids, which is secreted as a waste product of the aphids diet of phloem sap (Way, 1963). Production of honeydew is related to sap ingestion from plants so that plant compounds present in the sap, including some defensive compounds, are excreted through aphids' honeydew.

Additionally, plant products may survive the gastrointestinal tract and occur in animal faeces and then pass into degrading organisms in soil and soil water, constituting another possible route of exposure for some invertebrate species.

\subsubsection{Factors affecting RNAs physiological uptake}

Even if an organism is physically exposed to plant compounds through one of the routes described above, further steps may be required after the ingestion of plant parts in order to become physiologically exposed.

When RNA is present in the environmental matrices described above, invertebrate organisms are primarily physically exposed to nucleic acid through ingestion, although some other possible exposure routes have been proposed as well, such as passage through the integument (see Section 5.1). When successful ingestion occurs, cells of the gut surface may take up dsRNA and genes of these cells may become possible targets for interference. Several mechanisms (see Section 5.1) may allow further systemic transport of dsRNA and potentially target active genes in other tissues and organs. Therefore, as a consequence of these mechanisms, dsRNA or siRNA derived from its cleavage, can be found in cells and tissues remote from where they were introduced into the invertebrate.

A large body of research is available indicating that, at least in arthropod species, various barriers to physiological exposure exist. These include the potential degradation of dsRNA prior to ingestion , breakdown by nucleases in saliva and gastrointestinal tract (Allen and Walker, 2012; Christiaens et al., 2014; Garbutt et al., 2013; Liu et al., 2013; Prentice et al., 2017; Shukla et al., 2016; Wynant et al., 2014), degradation of dsRNA in the haemolymph (Christiaens et al., 2014), barriers to cellular uptake (Dalzell et al., 2011; Taning et al., 2016; Tomoyasu et al., 2008; Whyard et al., 2009) and the mechanisms of transport of dsRNA within the organism (Dalzell et al., 2011).

The present document has been produced and adopted by the bodies identified above as authors. This task has been carried out exclusively by the author(s) in the context of a contract between the European Food Safety Authority and the authors, awarded following a tender procedure. The present document is published complying with the transparency principle to which the Authority is subject. It may not be considered as an output adopted by the Authority. The European Food Safety Authority reserves its rights, view and position as regards the issues addressed and the conclusions reached in the present document, without prejudice to the rights of the authors. 
The presence of these barriers and their effectiveness in decreasing RNAi is not common to all invertebrates. In insects, it has become clear that in some orders, such as Lepidoptera (Garbutt et al., 2013; Liu et al., 2013; Shukla et al., 2016; Terenius et al., 2011), Hemiptera (Christiaens et al., 2014) and Homoptera (Wynant et al., 2014), nucleolytic degradation of dsRNA can negatively affect RNAi efficiency. Even in Coleoptera, which are generally considered highly sensitive to oral RNAi, examples of dsRNA degradation in the gut negatively impacting RNAi efficiency have been reported (Prentice et al., 2017). Also, oral intake studies have shown that uptake of RNA varies between different orders of arthropods. Many coleopteran species and nematodes will absorb RNA from plant and/or synthetic diets very efficiently, whereas for example in dipterans, oral RNAi can only be achieved when dsRNAs are formulated together with polymers or liposomes enhancing their cellular uptake and stability (Kumar et al., 2016; Taning et al., 2016; Whyard et al., 2009; Zhang et al., 2010). Furthermore, also in nematodes, research has revealed that differences in dsRNA uptake capacity and the ability to spread the silencing signal within the body can affect RNAi sensitivity by ingestion of dsRNA (Dalzell et al., 2011). A more detailed discussion on these topics, and other factors influencing RNAi efficiency in invertebrates, are presented in Section 5.1 and Section 5.3.

\subsubsection{Relevant publications from the systematic literature search}

From the initial list of 4,612 oral exposure studies, only 122 studies were considered relevant for Task 3 applying the criteria listed in Section 2.2.9. Among these, 117 studies concern exposure characterization (Appendix C) and environmental fate of RNA (Appendix D). Five additional studies in which transgenerational transmission of interference was studied in vitro via injection of RNAi, have also been selected as relevant for this task.

\subsubsection{Molecular characterisation in RNAi-based GM plants}

The primary exposure of organisms to environmental RNAs originating from transgenic plants is linked to their expression levels in the GM plant. Exposure studies on interfering RNAs have mostly focused on dsRNA, as little data was found on other interfering RNAs such as miRNA.

The plant-delivered expression level of an RNA-expressing construct can be measured by absolute or relative quantitative RT-PCR (qRT-PCR). The absolute quantification uses a calibration curve, while the latter technique, which is easier to perform, measures the relative change in mRNA expression levels of a target gene versus housekeeping genes (reference or control gene) (Livak and Schmittgen, 2001).

Analysis of in planta selected studies reveal that the expression of dsRNA is commonly assessed by qRT-PCR analysis and subsequently, transcript levels are determined with the comparative $\mathrm{C}_{\mathrm{T}}$ method using the formula $2^{-\Delta \Delta C t}$ (Abdellatef et al., 2015; Agrawal et al., 2015; Clément et al., 2009).

In some other studies, quantification of gene expression is calculated using the Pfaff equation, that takes into account primers efficiency (Fairbairn et al., 2007). Data is normally presented as the fold change in gene expression normalized to one or preferably, multiple selected endogenous reference genes.

For example, in Jaouannet et al. (2012) the relative expression of the exogenous hpMi-CRT (a hairpin RNA for M. incognita targeting calreticulin, a calcium binding-protein) in three Arabidopsis thaliana transgenic lines, was normalized to the expression of the NADH dehydrogenase (AT1G02020) housekeeping gene (Figure 5).

The present document has been produced and adopted by the bodies identified above as authors. This task has been carried out exclusively by the author(s) in the context of a contract between the European Food Safety Authority and the authors, awarded following a tender procedure. The present document is published complying with the transparency principle to which the Authority is subject. It may not be considered as an output adopted by the Authority. The European Food Safety Authority reserves its rights, view and position as regards the issues addressed and the conclusions reached in the present document, without prejudice to the rights of the authors. 


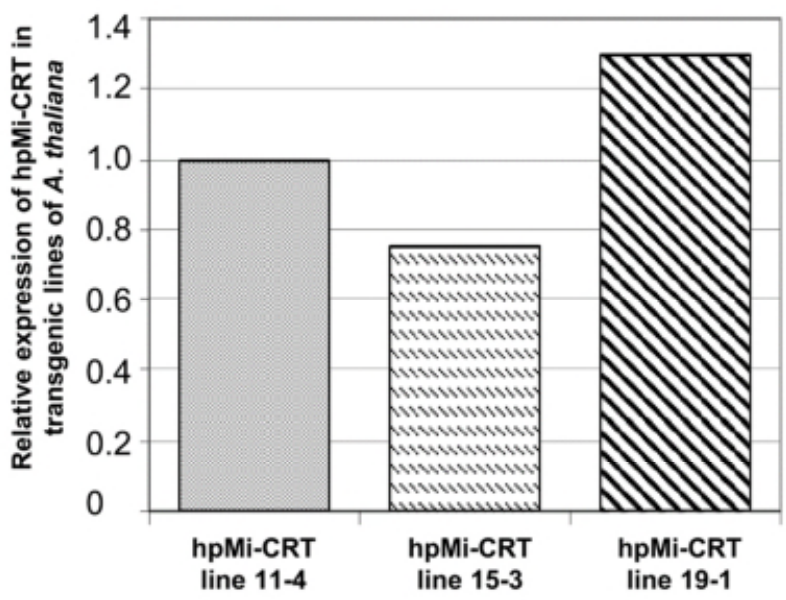

Figure 5: Expression patterns of hpMi-CRT in three Arabidopsis thaliana genetically modified lines. From (Jaouannet et al., 2012)

An effective method to quantify the expression level of dsRNA-containing transcripts expressed in transgenic plants, was developed by Armstrong et al. (2013). In this study, the authors reported for the first time a non-polymerase chain reaction-based quantitative assay for dsRNA-containing transcripts, using the QuantiGene kit. The assay platform does not require RNA purification and has a simple workflow based on RNA hybridization to specific DNA probes containing a fluorescent marker so that the expression level is used to fit a regression with the amount of dsRNA present in the sample. They used RNA from multiple maize tissue types and tissue lysates of the transgenic maize engineered to control western corn rootworm (event MON 87411), to quantify the expression of the DvSnf7 dsRNA transgene. Validation studies indicated that the assay was highly reproducible and sensitive to concentrations as low as $10 \mathrm{pg}$ of $\mathrm{dsRNA} / \mathrm{g}$ of fresh tissue.

Bachman et al. (2016) quantified the DvSnf7 dsRNA expression levels in different tissue types collected from MON 87411 plants across different sites, using the validated QuantiGene assay described above (Armstrong et al., 2013). The authors also calculated the expression levels of DvSnf7 dsRNA in micrograms of RNA per gram $(\mathrm{g})$ of fresh or dry weight tissue (Table 10).

The $D v S n f 7$ dsRNA maximum expression levels found in these studies were then used to determine the maximum expected environmental concentration (MEEC) for testing some groups of exposed arthropods including representative pollinators, natural enemies and soil biota. This is further discussed in Section 5.4 .

The same method was also used for efficiently measuring low concentrations of dsRNA in different soil and water samples (Albright et al., 2017; Dubelman et al., 2014; Fischer et al., 2016; Fischer et al., 2017).

The present document has been produced and adopted by the bodies identified above as authors. This task has been carried out exclusively by the author(s) in the context of a contract between the European Food Safety Authority and the authors, awarded following a tender procedure. The present document is published complying with the transparency principle to which the Authority is subject. It may not be considered as an output adopted by the Authority. The European Food Safety Authority reserves its rights, view and position as regards the issues addressed and the conclusions reached in the present document, without prejudice to the rights of the authors. 
Table 10: DvSnf7 dsRNA expression levels in different tissue type from MON 87411. (Modified from (Bachman et al., 2016)

\begin{tabular}{llc}
\hline Tissue type & Developmental stage & Range $\mathbf{~ g / g}$ \\
\hline Pollen (fwt) & VT-R1 & $0.056 \times 10^{-3}-0.224 \times 10^{-3}$ \\
Leaf (fwt) & V14-R1 & $5.40 \times 10^{-3}-33.8 \times 10^{-3}$ \\
Root (fwt) & V3-V4 & $1.74 \times 10^{-3}-8.00 \times 10^{-3}$ \\
Whole Plant (dwt) & V6-V8 & $33.0 \times 10^{-3}-106 \times 10^{-3}$ \\
Grain (dwt) & R6 & $0.056 \times 10^{-3}-0.175 \times 10^{-3}$ \\
\hline
\end{tabular}

fwt $=$ fresh weight, dwt $=$ dry weight

\subsubsection{Environmental fate in different matrices}

\section{Plant residues in soil}

Soils are chemically complex and spatially heterogeneous with surface-reactive particles (clay, sand, silt and humic substance) which can adsorb nucleic acids (Ranjard and Richaume, 2001). The presence of inorganic compounds and organic molecules on both soil particles and DNA molecules can influence the DNA adsorption and degradation and protect them against nuclease degradation (Pietramellara et al., 2009). The sorption of plant-derived insecticidal compounds may lead to irreversible adsorption to particle surfaces and a successive change of conformation upon adsorption. The adsorbed conformation of dsRNA depends on the adsorbent surface properties and solution chemistry (Parker and Sander, 2017). Existing knowledge on the fate of nucleic acids in soils indicate a low environmental persistence in most conditions (Levy-Booth et al., 2007; Poté et al., 2005). The fate of dsRNA will be affected by the biophysical characteristics of soil and sediments (e.g., hydrophobicity, salt concentration and $\mathrm{pH}$ ), in addition to other abiotic and biotic factors. The biodegradation of dsRNA, as with DNA degradation in soil, is likely to be mediated by microbialproduced ribonucleases, possibly in combination with plant-derived nucleases (Blum et al., 1997; Dubelman et al., 2014; Green, 1994; Poté et al., 2005).

The presence of several active moieties in the molecules may lead to the formation of metabolites that are challenging to identify in the soil. Extraction protocols for dsRNA from soil systems are less established and extraction systems are considered of low efficiency (Parker and Sander, 2017). Studies of degradation in soil were conducted in laboratory conditions to determine the potential biodegradation of a $D v S n f 7$ dsRNA transcript derived from a GM maize product that confers resistance to Diabrotica spp. (Dubelman et al., 2014). Maize tissues (shoots and roots) were added to different types of soil to simulate post-harvest conditions. Three agricultural soils differing in their physicochemical characteristics were used (silt loam, loamy sand and clay loam). Purified (in vitrotranscribed) DvSnf7 dsRNA was added to the soil $(0.3,1.5,7.5,37.5 \mu \mathrm{g} \mathrm{RNA} / \mathrm{g}$ soil in four different treatments) to increase its initial concentration. The GM and control (non-GM maize) materials were added to each soil and incubated at $22^{\circ} \mathrm{C}$ for 48 hours. Samples were collected at set time intervals during the incubation period, extracted, and analyzed using QuantiGene molecular analysis and insect bioassay (Southern Corn rootworm, D. undecimpunctata). The $\mathrm{DT}_{50}$ (half-life) values for DvSnf7 dsRNA in the three soils were 19, 28, and 15 hours based on QuantiGene, and 18, 29, and 14 hours based on the insect bioassay, respectively. The $\mathrm{DT}_{90}$ (time until $90 \%$ degradation) values for $D v S n f 7$ RNA in all three soils were 35 hours. DvSnf7 dsRNA was degraded and biological activity was undetectable within approximately two days after application to soil, regardless of texture, $\mathrm{pH}$, clay content and other soil differences. Furthermore, soil-incorporated DvSnf7 dsRNA was non-detectable in soil after 48 hours, as measured by QuantiGene, independently from the initial concentration, at levels ranging more than two orders of magnitude $(0.3,1.5,7.5$ and $37.5 \mathrm{mg} \mathrm{RNA} / \mathrm{g}$ soil).

The present document has been produced and adopted by the bodies identified above as authors. This task has been carried out exclusively by the author(s) in the context of a contract between the European Food Safety Authority and the authors, awarded following a tender procedure. The present document is published complying with the transparency principle to which the Authority is subject. It may not be considered as an output adopted by the Authority. The European Food Safety Authority reserves its rights, view and position as regards the issues addressed and the conclusions reached in the present document, without prejudice to the rights of the authors. 
A recent presentation given at the $14^{\text {th }}$ International Symposium on the Biosafety of Genetically Modified Organisms (ISBGMO14) ${ }^{2}$ described similar studies with two Brazilian soil samples, and reported similar levels of degradation of DvSnf7 dsRNA with time.

\section{Plant residues in water}

Nucleic acids usually persist in water for a limited time. Gulden et al. (2005) found that $\mathrm{DT}_{50}$ of plant DNA in leachate water ranged from 1.2 to 26.7 hours. Persistence in water of dsRNA was specifically investigated by Fischer et al. (2017) who conducted a study to measure the rate of biodegradation of DvSnf7 dsRNA in aerobic water sediment systems. In laboratory conditions, water from natural aquatic systems and sediments that varied in physical and chemical characteristics were treated with dsRNA by applying $60 \mu \mathrm{g}$ of $D v S n f 7$ dsRNA directly to the water column. The system consisted of about $90 \mathrm{~g}$ of sediment placed in the bottom of a $500 \mathrm{ml}$ graduated cylinders and covered with about $200 \mathrm{ml}$ of the corresponding water source (fresh water from a river or a lake). DvSnf7 dsRNA dissipated rapidly from the water phase and was undetectable within seven days in the diverse systems, when measured by QuantiGene and a sensitive insect bioassay with $D$. undecimpunctata. Degradation kinetics estimated a $\mathrm{DT}_{50}$ of less than three days and a time of about four days to reach $\mathrm{DT}_{90}$. When this laboratory setup was manipulated to obtain a "disturbed" system (i.e., the sediment was mixed into the water column following dsRNA application), $\mathrm{DT}_{50}$ and $\mathrm{DT}_{90}$ values for the water phases were less than one day regardless of the analytical method or the sediment-water origin.

Further analysis indicated that DvSnf7 dsRNA had $\mathrm{DT}_{50}$ values of less than six days in both sedimentfree systems containing natural water as well as systems with only sediment.

In a recent study, Albright et al. (2017) spiked a non-bioactive dsRNA into a column of water and sediment microcosm to mimic a run off of unbound dsRNA or transport of plant tissues. Dissipation of dsRNA in the water column and partitioning into sediment was determined. The dsRNA rapidly dissipated in the water column and was below the limit of detection after 96 hours. The levels detected in the sediment were not significant and may indicate a rapid degradation in the water column prior to partitioning to sediment. In both studies, a QuantiGene assay kit (Affymetrix) was used to determine dsRNA concentrations in the samples. This method has been shown to be capable of detecting low concentrations of dsRNA in soil regardless of sequence, molecular weight, or structure.

dsRNA stability under different temperatures and outdoor conditions was also the subject of the study conducted by Li et al. (2015), who obtained contrasting results. During laboratory experiments conducted in a water system, the authors observed a relative stability of dsRNA-containing solutions over a period of 8 hours under conditions of continuous UV exposure and high temperature (40$60^{\circ} \mathrm{C}$ ). When simulating outdoor conditions, independent of sunlight or shade, dsRNA began to gradually degrade after 1 month and the calculated half-life was 40 to 45 days. In this study, the dsRNA (fluorescent labelled) contents were detected using a NanoDrop ND-1000 spectrophotometer (NanoDrop ND-1000TM) and checked by agarose gel electrophoresis.

Considering the existing scientific literature on the subject, persistence of dsRNA in water is also expected to be short.

\section{Trophic chains from plants}

Transfer of RNA effectors between trophic levels is a mechanism which has been studied by several authors, in order to estimate possible impacts at higher trophic levels (i.e., consumers of different orders). However, no tritrophic studies demonstrating in vivo activity of plant derived RNA in prey on predator or parasitic species are available.

\footnotetext{
${ }^{2}$ Available at http://isbr.info/files/tinymce/uploaded/BOA\%20-\%2020062017\%20VA.pdf
}

The present document has been produced and adopted by the bodies identified above as authors. This task has been carried out exclusively by the author(s) in the context of a contract between the European Food Safety Authority and the authors, awarded following a tender procedure. The present document is published complying with the transparency principle to which the Authority is subject. It may not be considered as an output adopted by the Authority. The European Food Safety Authority reserves its rights, view and position as regards the issues addressed and the conclusions reached in the present document, without prejudice to the rights of the authors. 
Studies of dsRNA transfer from herbivores to natural enemies using non-plant derived RNA have been conducted. Garbian et al. (2012) discovered a bi-directional transfer of RNAi between honey bees and their parasitic mite Varroa destructor. The possible goal of the application of RNA interference mechanisms would be the control of this ubiquitous pest of apiaries. The experiments were originally conducted by using a "neutral" dsRNA carrying a segment of the gene for GFP. Adult bees were fed a sucrose solution containing dsRNA, which was also transferred to larvae via jelly produced by nurse bees and laid in cells where immatures develop. Subsequently, Varroa mites were allowed to feed on adult bees after sucrose feeding and examined after three days for direct dsRNA transfer. Exposure of mites to dsRNA from honeybee larvae was also induced by letting mites feed on larvae in cells (indirect transfer). Both direct and indirect successful transfer was confirmed via qRT-PCR. The authors also tested the bidirectional transfer of dsRNA from mites to bees by using Varroa individuals from previous experiments and allowing them to feed on new honeybee colonies which were not in contact with dsRNA. The dsRNA was detected in bees four days after infestation with dsRNA carrying mites, demonstrating that it was transferred to bees via parasitic activity.

After establishing the bidirectional transfer with marker genes, a number of dsRNAs were designed to target housekeeping genes of Varroa involved in cytoskeleton assembly, energy transfer, apoptosis inhibition, etc. The presence of phenotypic RNAi-mediated silencing effects was observed in experiments of direct and indirect transfer from bees. These results support the idea that dsRNA maintains its biological activity after across-species horizontal transfer. Diets containing different mixtures of dsRNA did not affect bee colony numbers, while a significant effect on the population of mites was detected.

Another demonstration of dsRNA transfer among different trophic levels is described by Itakura et al. (2009). In this study, the third trophic level was constituted by symbiotic protists of the termite, Coptotermes formosanus. The 21nt siRNAs designed for endoglucanases of the symbiotic protists Pseudotrichonympha grassii (PgEG), Holomastigotoides mirabile (HmEG), and Spirotrichonympha leidyi (SIEG) were used to silence protist genes through their termite hosts. In the experiment disorganization of $P$. grassii and $H$. mirabile occurred within a few days in the hindgut of the termites that had ingested PgEG and HmEG siRNAs suggesting that this mechanism could be used to impair the normal development of termite colonies.

\section{Transgenerational effects from plant derived RNA in herbivores}

A particular type of exposure route is the transmission of the effects of gene silencing due to dsRNA from a directly exposed herbivore to its progeny. This may lead to the exposure of larger numbers of individuals of the given species to the stressor (indirect exposure), increasing the overall frequency of exposure.

The first evidence of this phenomenon was found in C. elegans by Grishok et al. (2000b) who first investigated inheritance properties associated with silencing through exogenous dsRNA. Transmission of the interference effect occurred through a dominant extragenic agent. The activities of the RNAi pathway genes $r d e-1$ and $r d e-4$ were required for the formation of this interfering agent but were not needed for interference thereafter. Different genes, $r d e-2$ and mut-7 genes were required downstream for interference.

Burton et al. (2011) discovered that nuclear RNAi maintains heritable gene silencing and siRNA expression in the progeny of animals exposed to dsRNA. The epigenetic transmission pathway in the same species was further elucidated by Buckley et al. (2012) who discovered that the Argonaute protein HRDE-1 directs gene-silencing events in germ-cell nuclei that drive multigenerational RNAi inheritance.

The persistence of the epigenetic transmission was studied by Houri-Ze'evi et al. (2016) who showed that exposure to dsRNA activates a feedback loop, whereby gene-specific RNAi responses dictate the transgenerational duration of RNAi responses mounted against unrelated genes. Besides the induction of silencing, dsRNA-induced effects on the production of heritable endogenous small RNAs, which

The present document has been produced and adopted by the bodies identified above as authors. This task has been carried out exclusively by the author(s) in the context of a contract between the European Food Safety Authority and the authors, awarded following a tender procedure. The present document is published complying with the transparency principle to which the Authority is subject. It may not be considered as an output adopted by the Authority. The European Food Safety Authority reserves its rights, view and position as regards the issues addressed and the conclusions reached in the present document, without prejudice to the rights of the authors. 
regulate the expression of RNAi factors. Manipulating genes in this feedback pathway changed the duration of the heritable silencing.

However, the transgenerational transmission of silencing is not a general phenomenon and Ashe et al. (2015) found that antiviral RNA interference against natural Orsay virus infections is neither systemic nor transgenerational in C. elegans. While exogenous RNAi spreads throughout the organism and can be passed between generations, this phenomenon has not been observed for the endogenous RNA pathways.

Besides certain nematodes, evidence of transgenerational RNAi has also been found in certain insect species belonging to different orders including the coleopterans T. castaneum (Bucher et al., 2002), D. virgifera (Khajuria et al., 2015), Orchesella cincta (Konopova and Akam, 2014), the orthopteran G. bimaculatus (Mito et al., 2006), the hemipterans O. fasciatus (Liu and Kaufman, 2004), R. prolixus (Paim et al., 2013), M. persicae (Coleman et al., 2015), A. pisum (Mao et al., 2013), the blattodean B. germanica (Piulachs et al., 2010) and the hymenopterans A. rosae (Yoshiyama et al., 2013) and N. vitripennis (Lynch and Desplan, 2006).

Khajuria et al. (2015) described an RNAi mediated knockdown of two western corn rootworm developmental genes, (hunchback $(h b)$ and brahma $(b r m)$ ). After feeding adult females with dsRNAcontaining artificial diet a significant decrease in $h b$ and $b r m$ transcripts compared to negative control (treatment with GFP or water) was recorded in the tissues of eggs laid by the exposed females. Although total oviposition was not significantly affected, there was almost complete absence of hatching in the eggs collected from females exposed to dsRNA for either gene. This could be considered as a form of transgenerational effect, as the outcome of the silencing was expressed in embryos of the following generation, which was not directly exposed to the dsRNA.

Persistence and transgenerational effects of dsRNA were also studied on $M$. persicae feeding on GM plants (Coleman et al., 2015). The targeted gene was Rack1, a key component of various cellular processes. Maximal reduction of gene expression was $~ 70 \%$ and was achieved between four and eight days of exposure of the aphids to (dsRNA)-expressing Arabidopsis thaliana plants. Observations of the impact of RNAi was studied over three generations and the effects detected in the progenies lasted longer (12-14 days) than on the original individuals tested (approximately six days). Also, the effect on the population growth of aphids was more dramatic on the progeny $(60 \%$ decline in reproduction) than in the parental aphids (40\%).

\subsubsection{Conclusions}

The exposure of invertebrates to transgene products expressed in GM plant material can follow several pathways, which can be direct and indirect, and will therefore not be limited to the in-field situation or the growing season of the GM plant. Therefore, case-specific and local conditions are crucial for determining the likelihood and the extent of exposure of invertebrate organisms to dsRNAexpressing plant material.

While extensive data from studies of "first generation" GM crops (i.e., expression of proteins conferring insect resistance or herbicide tolerance) have clarified many aspects of exposure of target and NTOs, the evidence base on exposure pathways and environmental fate of interfering RNAs is very limited. Most of the available information focuses on SmartStax Pro maize expressing, among others, DvSnf7 dsRNA to control corn rootworms.

Scientific publications describing RNAi-expressing GM plants do not explicitly investigate potential exposure of invertebrate organisms. In most publications, information on dsRNA expression levels is limited to data on RNA silencing activity. Moreover, dsRNA expression levels in plants are rarely measured, probably because the primary objective of most of these studies is to investigate the effectiveness of RNA silencing. The evaluation of the effects of dsRNA was usually performed by qRT-PCR directly on the target gene expression of the invertebrate organism. In the studies reporting the results of dsRNA detection, relative values of expression compared to reference genes are usually

The present document has been produced and adopted by the bodies identified above as authors. This task has been carried out exclusively by the author(s) in the context of a contract between the European Food Safety Authority and the authors, awarded following a tender procedure. The present document is published complying with the transparency principle to which the Authority is subject. It may not be considered as an output adopted by the Authority. The European Food Safety Authority reserves its rights, view and position as regards the issues addressed and the conclusions reached in the present document, without prejudice to the rights of the authors. 
presented. Studies of in planta relative expression of dsRNA compared to housekeeping genes do not indicate actual amounts of dsRNA detected in plants. Therefore, these publications can only qualitatively orientate an exposure analysis. Similarly, only occasionally have detailed studies of expression in different plant tissues or different phenological stages of the plant life cycle been conducted. For example, Antonino de Souza Júnior et al. (2013) directly calculated the relative abundance of specific protease gene transcripts in $M$. incognita infesting dsRNA-expressing tobacco lines. The $M$. incognita $18 \mathrm{~S}$ ribosomal subunit (mi18S) was used for normalization of qRT-PCR data. The analysis was performed at different stages of the nematode life cycle and the results were presented as fold change referenced to the stage that had the smaller relative expression value to which was arbitrarily assigned the value 1 . Other examples where the efficiency of the silencing was directly evaluated on the target insect, include studies on the green peach aphid M. persicae (Pitino et al., 2011), lepidopteran pests S. littoralis (Apone et al., 2014) and H. armigera (Zhu et al., 2012) and the whitefly Bemisia tabaci (Raza et al., 2016).

The main exception pertains to the studies on characterization of GM maize MON 87411. In these publications, a quantitative determination of dsRNA expression in various plant parts was conducted using the molecular kit QuantiGene. This allows a sensitive detection of dsRNA in all plant tissues. Even with the availability of a relatively small amount of data, it can be inferred that in different transformation events with dsRNA, expression in plant parts was variable and the highest expression was detected in different tissues (from leaves to flowers) in the different studies.

Environmental persistence of dsRNA in decaying plant tissues and released from plants is expected to be limited. However, the use of different detection methods renders the available experimental results incomparable. The role of the different matrices in determining the environmental persistence of dsRNA (e.g., detailed information on the composition of soils, the presence of inorganic and organic compounds, as well as the microbial community of soil and sediments on site) might offer a better understanding of the possible persistence of dsRNA in soils.

No field studies were found in which the actual persistence of dsRNA derived from GM plants was evaluated on site. The published studies on the environmental degradation of dsRNA derived from GM plants are based on data obtained from soils studied under laboratory conditions.

The movement of dsRNA along trophic chains and the persistence of its biological activity have been shown in a few multitrophic systems. The likelihood of a biological effect is primarily linked to the uptake of dsRNA in taxonomically different organisms (see Section 5.1) and the efficiency of RNAi in the exposed organism (see. Section 5.3). Similarly, intergenerational effects have been studied in a few cases where exogenous dsRNA were observed in a number of generations after exposure. Due to the different mechanisms involved, predictions of similar effects in other biological systems are possible but yet to be demonstrated.

The case studies currently addressed by the scientific community are limited in terms of type of dsRNA used, plants species, NTOs selected, environmental conditions and agro-ecological landscape. Thus there is insufficient data to come to general conclusions concerning these factors. The availability of new dsRNA-expressing GM events will offer more opportunities for collecting specific relevant ecological data and filling the data gaps.

\subsubsection{References}

Abdellatef E, Will T, Koch A, Imani J, Vilcinskas A and Kogel K-H, 2015. Silencing the expression of the salivary sheath protein causes transgenerational feeding suppression in the aphid Sitobion avenae. Plant Biotechnology Journal 13, 849-857.

Agrawal A, Rajamani V, Reddy VS, Mukherjee SK, Bhatnagar RK, 2015. Transgenic plants overexpressing insect-specific microRNA acquire insecticidal activity against Helicoverpa armigera: an alternative to Bt-toxin technology. Transgenic Research 24, 791-801.

The present document has been produced and adopted by the bodies identified above as authors. This task has been carried out exclusively by the author(s) in the context of a contract between the European Food Safety Authority and the authors, awarded following a tender procedure. The present document is published complying with the transparency principle to which the Authority is subject. It may not be considered as an output adopted by the Authority. The European Food Safety Authority reserves its rights, view and position as regards the issues addressed and the conclusions reached in the present document, without prejudice to the rights of the authors. 
Albright VC, Wong CR, Hellmich RL, Coats JR, 2017. Dissipation of double-stranded RNA in aquatic microcosms. Environmental Toxicology and Chemistry 36, 1249-53.

Allen ML, Walker WB, 2012. Saliva of Lygus lineolaris digests double stranded ribonucleic acids. Journal of Insect Physiology 58, 391-6.

Álvarez-Alfageme F, Ortego F, Castañera P, 2009. Bt maize fed-prey mediated effect on fitness and digestive physiology of the ground predator Poecilus cupreus L. (Coleoptera: Carabidae). Journal of Insect Physiology 55, 144-50.

Antonino De Souza Júnior JD, Ramos Coelho R, Tristan Lourenço I, et al., 2013. Knocking-Down Meloidogyne incognita Proteases by Plant-Delivered dsRNA Has Negative Pleiotropic Effect on Nematode Vigor. PLoS ONE 8, e85364.

Apone F, Ruggiero A, Tortora A, et al., 2014. Targeting the Diuretic Hormone Receptor to Control the Cotton Leafworm, Spodoptera littoralis. Journal of Insect Science 14, 87.

Armstrong TA, Chen $\mathrm{H}$, Ziegler TE, et al., 2013. Quantification of Transgene-Derived Double-Stranded RNA in Plants Using the QuantiGene Nucleic Acid Detection Platform. Journal of Agricultural and Food Chemistry 61, 12557-64.

Arpaia S, Baldacchino F, Bosi S, et al., 2018. Evaluation of the potential exposure of butterflies to genetically modified maize pollen in protected areas in Italy. Insect Science in press.

Ashe A, Sarkies P, Le Pen J, Tanguy M, Miska EA, 2015. Antiviral RNA Interference against Orsay Virus Is neither Systemic nor Transgenerational in Caenorhabditis elegans. Journal of Virology 89, 12035-46.

Bachman PM, Huizinga KM, Jensen PD, et al., 2016. Ecological risk assessment for DvSnf7 RNA: A plant-incorporated protectant with targeted activity against western corn rootworm. Regulatory Toxicology and Pharmacology 81, 77-88.

Blum SaE, Lorenz MG, Wackernagel W, 1997. Mechanism of Retarded DNA Degradation and Prokaryotic Origin of DNases in Nonsterile Soils. Systematic and Applied Microbiology 20, 513-21.

Bucher G, Scholten J, Klingler M, 2002. Parental RNAi in Tribolium (Coleoptera). Current Biology 12, R85-R6.

Buckley BA, Burkhart KB, Gu SG, et al., 2012. A nuclear Argonaute promotes multigenerational epigenetic inheritance and germline immortality. Nature 489, 447-51.

Burton NO, Burkhart KB, Kennedy S, 2011. Nuclear RNAi maintains heritable gene silencing in Caenorhabditis elegans. Proceedings of the National Academy of Sciences of the United States of America 108, 19683-8.

Cain ML, Milligan BG, Strand AE, 2000. Long-distance seed dispersal in plant populations. American Journal of Botany 87, 1217-27.

Christiaens O, Swevers L, Smagghe G, 2014. DsRNA degradation in the pea aphid (Acyrthosiphon pisum) associated with lack of response in RNAi feeding and injection assay. Peptides 53, 307-14.

Clément M, Ketelaar T, Rodiuc N, et al., 2009. Actin-Depolymerizing Factor2-Mediated Actin Dynamics Are Essential for Root-Knot Nematode Infection of Arabidopsis. The Plant Cell 21, 2963-79.

Coleman AD, Wouters RHM, Mugford ST, Hogenhout SA, 2015. Persistence and transgenerational effect of plant-mediated RNAi in aphids. Journal of Experimental Botany 66, 541-8.

Dalzell JJ, Mcveigh P, Warnock ND, et al., 2011. RNAi Effector Diversity in Nematodes. PLOS Neglected Tropical Diseases 5, e1176.

The present document has been produced and adopted by the bodies identified above as authors. This task has been carried out exclusively by the author(s) in the context of a contract between the European Food Safety Authority and the authors, awarded following a tender procedure. The present document is published complying with the transparency principle to which the Authority is subject. It may not be considered as an output adopted by the Authority. The European Food Safety Authority reserves its rights, view and position as regards the issues addressed and the conclusions reached in the present document, without prejudice to the rights of the authors. 
Dubelman S, Fischer J, Zapata F, et al., 2014. Environmental Fate of Double-Stranded RNA in Agricultural Soils. PLOS ONE 9, e93155.

Eastham K, Sweet J, 2002. Genetically modified organisms (GMOs): the significance of gene flow through pollen transfer. European Environment Agency (EEA) report 28, 75 pp.

Ellstrand NC, Meirmans P, Rong J, et al., 2013. Introgression of Crop Alleles into Wild or Weedy Populations. Annual Review of Ecology, Evolution, and Systematics 44, 325-45.

Fairbairn DJ, Cavallaro AS, Bernard M, Mahalinga-Iyer J, Graham MW, Botella JR, 2007. Hostdelivered RNAi: an effective strategy to silence genes in plant parasitic nematodes. Planta 226, 1525-33.

Felke M, Langenbruch G-A, Feiertag S, Kassa A, 2010. Effect of Bt-176 maize pollen on first instar larvae of the Peacock butterfly (Inachis io) (Lepidoptera; Nymphalidae). Environ. Biosafety Res. 9, $5-12$.

Fischer JR, Zapata F, Dubelman S, Mueller GM, Jensen PD, Levine SL, 2016. Characterizing a novel and sensitive method to measure dsRNA in soil. Chemosphere 161, 319-24.

Fischer JR, Zapata F, Dubelman S, et al., 2017. Aquatic fate of a double-stranded RNA in a sedimentwater system following an over-water application. Environmental Toxicology and Chemistry 36, 727-34.

Garbian Y, Maori E, Kalev H, Shafir S, Sela I, 2012. Bidirectional Transfer of RNAi between Honey Bee and Varroa destructor: Varroa Gene Silencing Reduces Varroa Population. PLOS Pathogens 8, e1003035.

Garbutt JS, Bellés X, Richards EH, Reynolds SE, 2013. Persistence of double-stranded RNA in insect hemolymph as a potential determiner of RNA interference success: Evidence from Manduca sexta and Blattella germanica. Journal of Insect Physiology 59, 171-8.

Gathmann A, Wirooks L, Hothorn LA, Bartsch D, Schuphan I, 2006. Impact of Bt maize pollen (MON810) on lepidopteran larvae living on accompanying weeds. Mol Ecol 15.

Green PJ, 1994. The Ribonucleases of Higher Plants. Annual Review of Plant Physiology and Plant Molecular Biology 45, 421-45.

Grishok A, Tabara H, Mello CC, 2000. Genetic Requirements for Inheritance of RNAi in C. elegans. Science 287, 2494-7.

Gulden RH, Lerat S, Hart MM, et al., 2005. Quantitation of Transgenic Plant DNA in Leachate Water: Real-Time Polymerase Chain Reaction Analysis. Journal of Agricultural and Food Chemistry 53, 5858-65.

Harwood JD, Samson RA, Obrycki JJ, 2007. Temporal detection of Cry1Ab-endotoxins in coccinellid predators from fields of Bacillus thuringiensis corn. Bulletin of Entomological Research 97, 643-8.

Harwood JD, Wallin WG, Obrycki JJ, 2005. Uptake of Bt endotoxins by nontarget herbivores and higher order arthropod predators: molecular evidence from a transgenic corn agroecosystem. Molecular Ecology 14, 2815-23.

Hofmann F, Kruse-Plass M, Kuhn $U$, et al., 2016. Accumulation and variability of maize pollen deposition on leaves of European Lepidoptera host plants and relation to release rates and deposition determined by standardised technical sampling. Environmental Sciences Europe 28, 14.

Houri-Ze'evi L, Korem Y, Sheftel H, et al., 2016. A Tunable Mechanism Determines the Duration of the Transgenerational Small RNA Inheritance in C. elegans. Cell 165, 88-99.

Icoz I, Stotzky G, 2008. Fate and effects of insect-resistant Bt crops in soil ecosystems. Soil Biology and Biochemistry 40, 559-86.

The present document has been produced and adopted by the bodies identified above as authors. This task has been carried out exclusively by the author(s) in the context of a contract between the European Food Safety Authority and the authors, awarded following a tender procedure. The present document is published complying with the transparency principle to which the Authority is subject. It may not be considered as an output adopted by the Authority. The European Food Safety Authority reserves its rights, view and position as regards the issues addressed and the conclusions reached in the present document, without prejudice to the rights of the authors. 
Itakura S, Murayama S, Kamata Y, Tanaka H, Enoki A, 2009. RNA interference in symbiotic protists of the termite Coptotermes formosanus (Isoptera: Rhinotermitidae) through ingestion of siRNA by the host termite. Sociobiology 54, 77-87.

Jaouannet M, Magliano M, Arguel MJ, et al., 2012. The Root-Knot Nematode Calreticulin Mi-CRT Is a Key Effector in Plant Defense Suppression. Molecular Plant-Microbe Interactions 26, 97-105.

Khajuria C, Vélez AM, Rangasamy M, et al., 2015. Parental RNA interference of genes involved in embryonic development of the western corn rootworm, Diabrotica virgifera virgifera LeConte. Insect Biochemistry and Molecular Biology 63, 54-62.

Konopova B, Akam M, 2014. The Hox genes Ultrabithorax and abdominal-A specify three different types of abdominal appendage in the springtail Orchesella cincta (Collembola). Evodevo 5.

Kumar RD, Kumar SP, Gandhi MR, Al-Dhabi NA, Paulraj MG, Ignacimuthu S, 2016. Delivery of chitosan/dsRNA nanoparticles for silencing of wing development vestigial ( $\mathrm{vg}$ ) gene in Aedes aegypti mosquitoes. International Journal of Biological Macromolecules 86, 89-95.

Lang A, Ludy C, Vojtech E, 2004. Dispersion and deposition of Bt maize pollen in field margins / Pollenflug von Bf-Mais in angrenzende Feldränder. Zeitschrift für Pflanzenkrankheiten und Pflanzenschutz / Journal of Plant Diseases and Protection 111, 417-28.

Lavigne C, Klein EK, Vallée P, Pierre J, Godelle B, Renard M, 1998. A pollen-dispersal experiment with transgenic oilseed rape. Estimation of the average pollen dispersal of an individual plant within a field. Theoretical and Applied Genetics 96, 886-96.

Levy-Booth DJ, Campbell RG, Gulden RH, et al., 2007. Cycling of extracellular DNA in the soil environment. Soil Biology and Biochemistry 39, 2977-91.

Li H, Guan R, Guo H, Miao X, 2015. New insights into an RNAi approach for plant defence against piercing-sucking and stem-borer insect pests. Plant, Cell \& Environment 38, 2277-85.

Liu J, Smagghe G, Swevers L, 2013. Transcriptional response of BmToll9-1 and RNAi machinery genes to exogenous dsRNA in the midgut of Bombyx mori. Journal of Insect Physiology 59, 646-54.

Liu PZ, Kaufman TC, 2004. hunchback is required for suppression of abdominal identity, and for proper germband growth and segmentation in the intermediate germband insect Oncopeltus fasciatus. Development (Cambridge) 131, 1515-27.

Livak KJ, Schmittgen TD, 2001. Analysis of Relative Gene Expression Data Using Real-Time Quantitative PCR and the $2^{-\triangle \Delta C T}$ Method. Methods 25, 402-8.

Lynch JA, Desplan C, 2006. A method for parental RNA interference in the wasp Nasonia vitripennis. Nature Protocols 1, 486-94.

Mao J, Liu C, Zeng F, 2013. Hunchback is required for abdominal identity suppression and germband growth in the parthenogenetic embryogenesis of the pea aphid, Acyrthosiphon pisum. Archives of Insect Biochemistry and Physiology 84, 209-21.

Mccartney HA, Lacey ME, 1991. Wind dispersal of pollen from crops of oilseed rape (Brassica napus L.). Journal of Aerosol Science 22, 467-77.

Mito $T$, Okamoto $H$, Shinahara $W$, et al., 2006. Kruppel acts as a gap gene regulating expression of hunchback and even-skipped in the intermediate germ cricket Gryllus bimaculatus. Developmental Biology 294, 471-81.

Obrist LB, Dutton A, Romeis J, Bigler F, 2006. Biological Activity of Cry1Ab Toxin Expressed by Bt Maize Following Ingestion by Herbivorous Arthropods and Exposure of the Predator Chrysoperla carnea. BioControl 51, 31-48.

The present document has been produced and adopted by the bodies identified above as authors. This task has been carried out exclusively by the author(s) in the context of a contract between the European Food Safety Authority and the authors, awarded following a tender procedure. The present document is published complying with the transparency principle to which the Authority is subject. It may not be considered as an output adopted by the Authority. The European Food Safety Authority reserves its rights, view and position as regards the issues addressed and the conclusions reached in the present document, without prejudice to the rights of the authors. 
Paim RMM, Araujo RN, Lehane MJ, Gontijo NF, Pereira MH, 2013. Long-term effects and parental RNAi in the blood feeder Rhodnius prolixus (Hemiptera; Reduviidae). Insect Biochemistry and Molecular Biology 43, 1015-20.

Parker KM, Sander M, 2017. Environmental Fate of Insecticidal Plant-Incorporated Protectants from Genetically Modified Crops: Knowledge Gaps and Research Opportunities. Environmental Science \& Technology 51, 12049-57.

Pietramellara G, Ascher J, Borgogni F, Ceccherini MT, Guerri G, Nannipieri P, 2009. Extracellular DNA in soil and sediment: fate and ecological relevance. Biology and Fertility of Soils 45, 219-35.

Pitino M, Coleman AD, Maffei ME, Ridout CJ, Hogenhout SA, 2011. Silencing of Aphid Genes by dsRNA Feeding from Plants. PLOS ONE 6, e25709.

Piulachs MD, Pagone V, Bellés $X$, 2010. Key roles of the Broad-Complex gene in insect embryogenesis. Insect Biochemistry and Molecular Biology 40, 468-75.

Poté J, Rossé P, Rosselli W, Van VT, Wildi W, 2005. Kinetics of mass and DNA decomposition in tomato leaves. Chemosphere 61, 677-84.

Poté J, Ackermann R, Wildi W (2009) Plant leaf mass loss and DNA release in freshwater sediments. Ecotoxicol Environ Saf 72:1378-1383

Prentice K, Christiaens O, Pertry I, et al., 2017. RNAi-based gene silencing through dsRNA injection or ingestion against the African sweet potato weevil Cylas puncticollis (Coleoptera: Brentidae). Pest Management Science 73, 44-52.

Ranjard L, Richaume A, 2001. Quantitative and qualitative microscale distribution of bacteria in soil. Research in Microbiology 152, 707-16.

Raps A, Kehr J, Gugerli P, Moar WJ, Bigler F, Hilbeck A, 2001. Immunological analysis of phloem sap of Bacillus thuringiensis corn and of the nontarget herbivore Rhopalosiphum padi (Homoptera: Aphididae) for the presence of Cry1Ab. Molecular Ecology 10, 525-33.

Raza A, Malik HJ, Shafiq M, et al., 2016. RNA Interference based Approach to Down Regulate Osmoregulators of Whitefly (Bemisia tabaci): Potential Technology for the Control of Whitefly. PLOS ONE 11, e0153883.

Rosi-Marshall EJ, Tank JL, Royer TV, et al., 2007. Toxins in transgenic crop byproducts may affect headwater stream ecosystems. Proceedings of the National Academy of Sciences 104, 16204-8.

Shi Y, Wang MB, Powell KS, et al., 1994. Use of the rice sucrose synthase-1 promoter to direct phloem-specific expression of $\beta$-glucuronidase and snowdrop lectin genes in transgenic tobacco plants. Journal of Experimental Botany 45, 623-31.

Shukla JN, Kalsi M, Sethi A, et al., 2016. Reduced stability and intracellular transport of dsRNA contribute to poor RNAi response in lepidopteran insects. RNA Biology 13, 656-69.

Svobodová Z, Shu Y, Skoková Habuštová O, Romeis J, Meissle M, 2017. Stacked Bt maize and arthropod predators: exposure to insecticidal Cry proteins and potential hazards. Proceedings of the Royal Society B: Biological Sciences 284.

Taning CNT, Christiaens O, Berkvens N, Casteels H, Maes M, Smagghe G, 2016. Oral RNAi to control Drosophila suzukii: laboratory testing against larval and adult stages. Journal of Pest Science 89, 803-14.

Tank JL, Rosi-Marshall EJ, Royer TV, Whiles MR, Griffiths NA, Frauendorf TC, Treering DJ (2010) Occurrence of maize detritus and a transgenic insecticidal protein (Cry1Ab) within the stream network of an agricultural landscape. Proc Natl Acad Sci USA 107:17645-17650

The present document has been produced and adopted by the bodies identified above as authors. This task has been carried out exclusively by the author(s) in the context of a contract between the European Food Safety Authority and the authors, awarded following a tender procedure. The present document is published complying with the transparency principle to which the Authority is subject. It may not be considered as an output adopted by the Authority. The European Food Safety Authority reserves its rights, view and position as regards the issues addressed and the conclusions reached in the present document, without prejudice to the rights of the authors. 
Terenius O, Papanicolaou A, Garbutt JS, et al., 2011. RNA interference in Lepidoptera: An overview of successful and unsuccessful studies and implications for experimental design. Journal of Insect Physiology 57, 231-45.

Tomoyasu Y, Miller SC, Tomita S, Schoppmeier M, Grossmann D, Bucher G, 2008. Exploring systemic RNA interference in insects: a genome-wide survey for RNAi genes in Tribolium. Genome Biology 9, R10-R.

Treu R, Emberlin J, Association S, 2000. Pollen Dispersal in the Crops Maize (Zea Mays), Oil Seed Rape (Brassica Napus Ssp Oleifera), Potatoes (Solanum Tuberosum), Sugar Beet (Beta Vulgaris Ssp. Vulgaris) and Wheat (Triticum Aestivum): Evidence from Publications. Soil Association.

Verkerk KJ, 2004. Manipulation of tritrophic interactions in IPM. In: Koul O, Dhaliwal GS, Cuperus GW, eds. Integrated Pest Management: Potential, constraints and challenges. UK: CABI Publishing, 5572.

Way MJ, 1963. Mutualism Between Ants and Honeydew-Producing Homoptera. Annual Review of Entomology 8, 307-44.

Whyard $S$, Singh $A D$, Wong $S, 2009$. Ingested double-stranded RNAs can act as species-specific insecticides. Insect Biochemistry and Molecular Biology 39, 824-32.

Wynant N, Santos D, Verdonck R, Spit J, Van Wielendaele P, Vanden Broeck J, 2014. Identification, functional characterization and phylogenetic analysis of double stranded RNA degrading enzymes present in the gut of the desert locust, Schistocerca gregaria. Insect Biochemistry and Molecular Biology 46, 1-8.

Yoshiyama N, Tojo K, Hatakeyama M, 2013. A survey of the effectiveness of non-cell autonomous RNAi throughout development in the sawfly, Athalia rosae (Hymenoptera). Journal of Insect Physiology 59, 400-7.

Zapiola ML, Campbell CK, Butler MD, Mallory-Smith CA, 2008. Escape and establishment of transgenic glyphosate-resistant creeping bentgrass Agrostis stolonifera in Oregon, USA: a 4-year study. Journal of Applied Ecology 45, 486-94.

Zhang X, Zhang J, Zhu KY, 2010. Chitosan/double-stranded RNA nanoparticle-mediated RNA interference to silence chitin synthase genes through larval feeding in the African malaria mosquito (Anopheles gambiae). Insect Molecular Biology, 19, 683-693.

Zhu J-Q, Liu S, Ma Y, et al., 2012. Improvement of Pest Resistance in Transgenic Tobacco Plants Expressing dsRNA of an Insect-Associated Gene ECR. PLOS ONE 7, e38572.

The present document has been produced and adopted by the bodies identified above as authors. This task has been carried out exclusively by the author(s) in the context of a contract between the European Food Safety Authority and the authors, awarded following a tender procedure. The present document is published complying with the transparency principle to which the Authority is subject. It may not be considered as an output adopted by the Authority. The European Food Safety Authority reserves its rights, view and position as regards the issues addressed and the conclusions reached in the present document, without prejudice to the rights of the authors. 


\subsection{Factors influencing silencing efficiency of dsRNA, siRNA and miRNA delivered orally in arthropods, nematodes, annelids and molluscs (Task 4)}

\subsubsection{Introduction}

While RNAi experiments have been performed since the 1980s, reported as antisense, quelling or cosuppression experiments in nematodes, fungi and plants, the true mechanism of RNAi was not known until Fire et al. (1998) demonstrated that this post-transcriptional gene silencing phenomenon in the nematode $C$. elegans is actually initiated by dsRNA, rather than sense or antisense RNA. After this discovery, researchers started using this technique in $C$. elegans on a large scale, mainly for research purposes. Initially, microinjection was used as a delivery method, but a feeding delivery was soon discovered to be functional and was adopted in many studies as an easy and efficient delivery method (Fraser et al., 2000; Timmons and Fire, 1998).

Soon after the discovery of RNAi in nematodes, the first RNAi-experiments in the model insect, the fruitfly $D$. melanogaster were reported, showing that the technique can be used in these flies for gene knockdown by microinjection (Kennerdell and Carthew, 1998; Misquitta and Paterson, 1999; Willert et al., 1999). This was confirmed in other arthropod species such as the coleopteran insect $T$. castaneum. Feeding experiments in D. melanogaster, using naked dsRNA, did not seem to work initially (Perrimon et al., 2010; Whyard et al., 2009). However, they were found to be successful in some other arthropods. One of the first reported successful oral RNAi studies was that in the tick I. scapularis (Soares et al., 2005) where researchers managed to silence the isac gene using capillary feeding of dsRNA. Not long after, Turner et al. (2006) showed that feeding dsRNA to the lepidopteran light brown apple moth Epiphyas postvittana could induce silencing, but only when feeding very high doses of dsRNA. Many more studies on arthropods, mainly insects, followed and researchers quickly recognised the potential of this technique for pest control. The first paper in this context was published by Baum et al. (2007), showing that feeding dsRNA to two beetle species, the Colorado potato beetle $L$. decemlineata and the Western corn rootworm $D$. virgifera, could be a very potent insecticidal pest control strategy as both species proved to be very sensitive to oral RNAi.

These early studies in nematodes and arthropods already gave an indication of the variability of RNAi efficiency in invertebrates. While $L$. decemlineata and $D$. virgifera seemed to exhibit a similar sensitivity to RNAi, as is the case for $C$. elegans, this was not the case for many dipteran and lepidopteran species for example, which were either insensitive or required to be exposed to very high doses of dsRNA in order to achieve any gene silencing. In this Section, we discuss the available data on RNAi sensitivity and efficiency in invertebrates, mainly focusing on feeding RNAi, and also discuss the factors that influence RNAi efficiency. Several factors, for example stability of dsRNA in the invertebrate body, the cellular uptake of dsRNA from the gut, the RNAi core machinery and the possible effect of viral infections have been identified by various authors and are discussed here.

\subsubsection{RNAi efficiency in invertebrates}

In this section, we review oral RNAi experiments in different invertebrate taxonomic clades in order to give an indication on the sensitivity of RNAi in these groups and the doses and concentrations that were required to elicit efficient silencing responses. An overview is given in Table 11 and Table 12.

\subsubsection{Nematodes}

\section{Caenorhabditis elegans}

The success of feeding RNAi in animals was first reported in C. elegans (Timmons and Fire, 1998). In this study, E. coli bacteria were transformed to express dsRNA specifically targeting three genes (unc-22, fem-1 and $g f p$ ) and were fed to the $C$. elegans worms. This was a logical delivery method,

The present document has been produced and adopted by the bodies identified above as authors. This task has been carried out exclusively by the author(s) in the context of a contract between the European Food Safety Authority and the authors, awarded following a tender procedure. The present document is published complying with the transparency principle to which the Authority is subject. It may not be considered as an output adopted by the Authority. The European Food Safety Authority reserves its rights, view and position as regards the issues addressed and the conclusions reached in the present document, without prejudice to the rights of the authors. 
since $C$. elegans nematodes naturally feed on these bacteria. Feeding of these transformed $E$. coli led to a reversible gene silencing, similar to that induced by microinjected dsRNA. Furthermore, the effect was observed in somatic tissues, indicating transport of the RNAi silencing signal from the gut to other parts of the body. However, the authors reported that this feeding approach was less effective compared to microinjection, since only a part of the treated population exhibited the silencing phenotypes and the effects were found to be less severe. Indeed, while silencing unc-22 led to a specific phenotype in $85 \%$ of the treated individuals, for fem-1 and gfp this was only $43 \%$ and $12 \%$, respectively (Timmons and Fire, 1998). Nevertheless, given the practical advantages of this delivery method compared to microinjection, feeding dsRNA-expressing bacteria as a delivery method became widely adopted for RNAi research in $C$. elegans and protocols were also improved, leading to higher efficiencies. Kamath et al. (2001) presented an optimized feeding method which leads to phenotypes which were at least as strong and frequent as those achieved by dsRNA microinjection. They used a specific strain of $E$. coli, called the HT115 strain, in which RNase III expression had been knocked out, to prevent dsRNA degradation inside the bacteria. Furthermore, they also optimized the expression induction conditions, finding that ispropyl $\beta$-D-1-thiogalactopyranoside (IPTG) induction at room temperature overnight leads to optimal RNAi results in the C. elegans worms $(95-100 \%$ phenotype frequency).

One of the disadvantages of this bacterial feeding approach is the difficulty to actually assess the quantitative efficiency, since the amount of dsRNA eventually taken up via these bacteria is unknown. Only a minority of oral RNAi studies in $C$. elegans have been conducted using known concentrations of naked dsRNA in the form of a soaking solution (Annex 2). The soaking solution delivery was first reported by Maeda et al. (2001) who soaked L4 worms in dsRNA at concentrations of $1-5 \mu \mathrm{g} / \mu \mathrm{L}$, resulting in $100 \%$ RNAi phenotypes in the F1 progeny. All soaking RNAi studies in C. elegans that were retrieved in the systematic literature search of this project used dsRNA concentrations in the $\mu \mathrm{g} / \mu \mathrm{L}$ range. No experiments using lower concentrations were found. Many studies using soaking methods reported RNAi phenotypes in most treated worms. For example, silencing $h s p$ genes in C. elegans using the protocol described by Maeda et al. (2001) caused developmental retardation in $73.8 \%$ of the treated population after 72 hours (Al-Amin et al., 2016). When the stip1 gene was silenced in L4 individuals, a 100\% embryonic lethality of the progeny was observed (Ji et al., 2007). Silencing the par-5 gene in L4 larvae caused sterility in $66 \%$ of the $F_{1}$ progeny (Morton et al., 2002). Unfortunately, most of these studies did not include qRT-PCR data, which would allow us to evaluate silencing efficiency at the transcript level. However, the widespread phenotypical effects do indicate that the soaking exposure to dsRNA can efficiently trigger RNAi, albeit at relatively high concentrations of $1-5 \mu \mathrm{g} / \mu \mathrm{L}$. In our systematic literature search, no studies were retrieved where miRNA was fed to nematodes. Interesting to note here is that several natural isolates of $C$. elegans (and other Caenorhabditis species) have been found which have defects in their RNAi pathways either in somatic cells or in the germline (Felix, 2008; Tijsterman et al., 2002). So, variability of RNAi efficiency can exist between different strains of $C$. elegans.

\section{Other nematode species}

As already mentioned in Sections 5.1 when discussing uptake mechanisms, some variability has been observed in RNAi sensitivity in nematode species (Felix, 2008). In the Caenorhabditis species C. briggsae, $C$. remanei and C. brenneri, exposure to oral intake of dsRNA does not elicit as effective silencing response as injected dsRNA. In the former two species, it has been reported that this is most likely due to the lack of sid-2 genes in their genome, as the $C$. elegans Sid-2 protein is able to rescue functional feeding RNAi in these nematodes (Felix, 2008; Nuez and Felix, 2012; Winston et al., 2007). Also in other nematodes, RNAi sensitivity seems to be variable. Felix (2008) compared RNAi efficacy in 17 different nematode species and reported that oral RNAi is ineffective in six species (C. briggsae, C. remanei, C. brenneri, O. tipulae, P. pacificus and Strongyloides stercoralis) and is variably or moderately effective in three others $(H$. contortus, O. ostertagi and $B$. malayi) (Aboobaker and Blaxter, 2003; Aboobaker and Blaxter, 2004; Geldhof et al., 2006; Lok, 2007; Louvet-Vallée et al., 2003; Pires-daSilva and Sommer, 2004; Visser et al., 2006; Winston et al., 2007). These include

The present document has been produced and adopted by the bodies identified above as authors. This task has been carried out exclusively by the author(s) in the context of a contract between the European Food Safety Authority and the authors, awarded following a tender procedure. The present document is published complying with the transparency principle to which the Authority is subject. It may not be considered as an output adopted by the Authority. The European Food Safety Authority reserves its rights, view and position as regards the issues addressed and the conclusions reached in the present document, without prejudice to the rights of the authors. 
both animal and plant parasitic species. Furthermore, the free-living mycophagous nematode Aphelenchus avenae has also been found to be recalcitrant to oral RNAi (Reardon et al., 2010). In several grassland soil nematodes, insensitivity to RNAi was reported, including after dsRNA-injection (Wheeler et al., 2012). A meta study looking into the RNAi-pathway related genes showed that Caenorhabditis sp. have an expanded RNAi-related gene repertoire compared to parasitic nematodes and that especially the genes related to uptake and spread of the RNAi signal in C. elegans are poorly conserved in parasitic species (Dalzell et al., 2011). However, these authors indicated that, despite quantitative differences in terms of RNAi-related genes, there was no qualitative difference in terms of functional groups that were represented in these nematode species. Furthermore, they could also not identify a link between RNAi effector repertoire and RNAi functionality in parasitic nematodes.

Despite this observed RNAi variability and the differences with $C$. elegans in terms of RNAi effector repertoire, successful oral or soaking RNAi has been reported in many nematode species. Contrary to C. elegans, most RNAi studies in other nematode species used a non-bacterial feeding or soaking method. We found 34 studies using artificial diet or soaking and over 40 studies reporting successful RNAi using in planta delivery methods, either using GM plants or viral-/bacterial-mediated plant delivery. In most of the retrieved in vitro feeding or soaking studies, dsRNA concentrations in the $1-10 \mu \mathrm{g} / \mu \mathrm{L}$ range, similar to those in $C$. elegans, were used (Annex 2). In a study by Bakhetia et al. (2007), soaking of $\mathrm{J} 2$ stage $H$. glycines in a $2 \mu \mathrm{g} / \mu \mathrm{L}$ dsRNA solution targeting eng- 1 led to severe silencing at the transcript level for at least five days. Between day five and 10 post-soaking, the gene expression recovered. In another study, targeting pat-10 and unc-87 in Pratylenchus coffeae also led to a strong silencing response after 24 hours soaking in a $1 \mu \mathrm{g} / \mu \mathrm{L}$ dsRNA solution. However, recovery of expression happened quickly, in less than 24 hours. Phenotypically, silencing of both genes led to severe impairment of mobility of the nematodes (Joseph et al., 2012). In the pinewood nematode B. xylophilus, targeting vap- 1 by soaking in a $4 \mu \mathrm{g} / \mu \mathrm{L}$ solution led to a silencing efficiency of $48 \pm 3 \% 24$ hours after the start of the treatment, which resulted in a significantly reduced migration rate (Kang et al., 2012). Similar RNAi successes were also reported, for example in Ditylenchus destructor, Panagrolaimus superbus, Globodera rostochiensis, $R$. similis (Peng et al., 2014; Reardon et al., 2010; Rehman et al., 2009; Wang et al., 2016b). Interestingly, two studies reported successful RNAi silencing using $50 \mathrm{ng} / \mu \mathrm{L}$ and $25 \mathrm{ng} / \mu \mathrm{L}$ siRNA soaking solutions. The former observed a strong silencing effect lasting between 48-72 hours before transcript expression recovered upon soaking of M. graminicola in the siRNA solution (Nsengimana et al., 2013). Phenotypically, the RNAi-treated nematodes in this study were severely affected in their mobility after silencing of the pat-10 and unc-87 genes. In the latter study, Heterodera avenae were soaked in a $25 \mathrm{ng} / \mu \mathrm{L}$ siRNA solution. The researchers did not look at the gene's transcript levels in these soaking experiments, but did report significant mortality in the targeted nematodes (Zheng et al., 2015). Based on the few studies where nematodes were exposed to siRNA, rather than long dsRNA (Ma et al., 2011; Nsengimana et al., 2013; Zheng et al., 2015), there does not seem to be an obvious difference in efficiency between both molecules. Ma et al. (2011) noticed large differences in RNAi silencing efficiency between different siRNAs targeting a different region of the gene.

In the past decade, several studies have also shown that nematodes can be targeted for efficient RNAi through plants, either via a GM plant or transiently introduced dsRNA or siRNA in the plant (Annex 2). A study where hairpin dsRNAs targeting the M. incognita splicing factor and integrase genes were expressed in tobacco plants showed a dramatic decrease in the number of root knots, number of females per knot and number of egg masses on the plant, compared to the non-RNAi plant (Yadav et al., 2006). Furthermore, the authors also confirmed functional RNAi by semi-quantitative PCR, showing a complete absence of target mRNA. In another study, targeting the 16D10 gene in four different root-knot nematodes (M. incognita, Meloidogyne javanica, Meloidogyne arenaria and Meloidogyne hapla) through in vitro soaking and through in vivo Arabidopsis, in planta RNAi caused significant silencing at the transcript and protein level (Huang et al., 2006). Another study investigated both soaking and in planta delivery in Meloidogyne species. Treatment of freshly hatched M. incognita $\mathrm{J} 2 \mathrm{~s}$ in a $1 \mu \mathrm{g} / \mu \mathrm{L}$ dsRNA solution caused $93-97 \%$ silencing at the transcript level and a

The present document has been produced and adopted by the bodies identified above as authors. This task has been carried out exclusively by the author(s) in the context of a contract between the European Food Safety Authority and the authors, awarded following a tender procedure. The present document is published complying with the transparency principle to which the Authority is subject. It may not be considered as an output adopted by the Authority. The European Food Safety Authority reserves its rights, view and position as regards the issues addressed and the conclusions reached in the present document, without prejudice to the rights of the authors. 
$65-69 \%$ reduction at the protein level. In the in vivo experiments, dsRNA-expressing plants showed a $63-96 \%$ reduction in number of galls and a decrease in gall size for several Meloidogyne species. Furthermore, a 66-93\% reduction in number of eggs per gram root was reported as well (Huang et al., 2006). These experiments indicate that in planta exposure to target-specific dsRNAs can cause a highly efficient silencing in these plant-parasitic nematodes.

Table 11: Overview of reported SRNA concentrations or single doses in genetically modified plants, in vitro feeding and soaking studies to elicit successful RNAi gene silencing in nematodes

\begin{tabular}{lllccl}
\hline Class & Order & In planta & $\begin{array}{c}\text { In vitro } \\
\text { feeding }\end{array}$ & $\begin{array}{c}\text { In vitro } \\
\text { soaking }\end{array}$ & Comments \\
\hline Adenophorea & Trichocephalida & - & - & $0.05 \mu \mathrm{g} / \mu \mathrm{L}$ & $\begin{array}{l}\text { Only one soaking study } \\
\text { available (Chen et al., 2012) } \\
\text { Secernentea }\end{array}$ \\
\cline { 2 - 4 } & Rhabditida & - & $1-5 \mu \mathrm{g} / \mu \mathrm{L}$ & $1-10 \mu \mathrm{g} / \mu \mathrm{L}$ & $\begin{array}{l}\text { Most studies using bacterial } \\
\text { production systems (feeding } \\
\text { or soaking) }\end{array}$ \\
& Tylenchida & Sni & $1-2 \mu \mathrm{g} / \mu \mathrm{L}$ & $\begin{array}{c}0.05-10 \\
\mu \mathrm{g} / \mu \mathrm{L}\end{array}$ & $\begin{array}{l}\text { Most studies using in planta } \\
\text { delivery systems }\end{array}$ \\
\hline
\end{tabular}

Sni: Succesful silencing but no information on concentration or expression level in the plant

- No studies found

\subsubsection{Arthropods}

In our systematic literature search, we retrieved nearly 350 studies in which dsRNA, siRNA or miRNA was delivered to arthropod species via feeding, soaking or through a topical application (Annex 2). In the majority of these studies it was achieved with dsRNA molecules, while siRNA or miRNA studies were less common. The majority of studies were done using insect species and the number of Crustacea or Chelicerata oral RNAi studies is limited. An overview of the doses or concentrations that elicited successful silencing in arthropods is given in Table 12.

\subsubsection{Insecta}

In insects, a diverse range of oral delivery methods have been used for sRNAs, including soaking, delivery through plants, topical application or mixing sRNAs with artificial diet. Not surprisingly, given the interest in RNAi for crop protection, most of the information available in this context of oral RNAi concerns pest organisms, as can be seen in Annex 2. In this section, we give an overview of the recorded RNAi sensitivity in insect species. While we mention possible factors explaining the differences in RNAi sensitivity in certain species, these factors are discussed in much more detail in Section 5.3.3 of this Technical Report .

\section{Diptera}

The first insect in which RNAi was attempted was the dipteran D. melanogaster. This was not surprising, given the fact that it has been considered a model insect for decades and that its genome was sequenced in the late 1990s (Adams et al., 2000). While RNAi by injection and by soaking of dechorionated embryos was functional in D. melanogaster (Baqri et al., 2006; Dzitoyeva et al., 2001; Dzitoyeva et al., 2003; Eaton et al., 2002; Misquitta et al., 2008; Schmid et al., 2002), this was not the case when flies were exposed to dsRNA orally (Perrimon et al., 2010; Whyard et al., 2009). However, Whyard et al. (2009) demonstrated that adding a liposomic transfection agent to the dsRNA significantly increased oral RNAi efficiency in these fruit flies, leading to the hypothesis that Drosophila lacks a functional dsRNA cellular uptake mechanism, possibly related to the lack of any Sid-1-like genes in its genome (Feinberg and Hunter, 2003; Miller et al., 2008; Tomoyasu et al., 2008; Ulvila et al., 2006; Whyard et al., 2009). In a close relative, Drosophila suzukii, similar findings were reported for the use of naked dsRNA and liposome-coated dsRNA (Taning et al., 2016b). A single naked dsRNA feeding event to $D$. suzukii larvae did not result in any observable silencing or

The present document has been produced and adopted by the bodies identified above as authors. This task has been carried out exclusively by the author(s) in the context of a contract between the European Food Safety Authority and the authors, awarded following a tender procedure. The present document is published complying with the transparency principle to which the Authority is subject. It may not be considered as an output adopted by the Authority. The European Food Safety Authority reserves its rights, view and position as regards the issues addressed and the conclusions reached in the present document, without prejudice to the rights of the authors. 
phenotype, while coating the dsRNA with liposomes caused up to $42 \pm 2 \%$ silencing on transcript level and an increase of up to $42 \pm 7 \%$ mortality compared to the control (Taning et al., 2016b). Furthermore, another study reported that continuous delivery to $D$. suzukii through dsRNA-expressing yeast symbionts also resulted in a significant RNAi silencing effect (up to $76 \%$ on transcript level) which led to decreased survival, mobility and fecundity of the fruit flies (Murphy et al., 2016). In total, only three studies were retrieved in the systematic literature search on oral RNAi in Drosophila and none of them reported successful RNAi using naked dsRNA.

In another genus of fruit flies, Bactrocera, we found eight studies investigating or using oral RNAi. Interestingly, all these studies targeted adult flies. No RNAi studies on Bactrocera larvae were found. Concentrations used in these studies were $0.5-2.0 \mu \mathrm{g} / \mu \mathrm{L}$ in most cases. A study by Li et al. (2011) reported successful silencing of four genes in $B$. dorsalis after continuous feeding on artificial diet containing $2 \mu \mathrm{g} / \mu \mathrm{L}$ dsRNA. Remarkably, the RNAi silencing effect only lasted for a short time (1-3 days) and in some cases, it was followed by a strong upregulation of the gene expression to a much higher level than the non-dsRNA fed control, while still being fed the target dsRNA. In another study, researchers fed dsRNA targeting the rpl19 gene to $B$. dorsalis and $B$. minax, and also achieved significant silencing 24 hours after feeding. For B. dorsalis, a silencing efficiency of $54 \%$ and $69 \%$ was observed at $0.5 \mu \mathrm{g} / \mu \mathrm{L}$ and $1 \mu \mathrm{g} / \mu \mathrm{L}$ dsRNA concentrations, respectively. For $B$. minax, this was $90 \%$ and $91 \%$ (Chen et al., 2015). Yi et al. (2013) reported successful silencing of the CSP2 gene in B. dorsalis, after continuous feeding on an artificial diet containing $1 \mu \mathrm{g} / \mu \mathrm{L}$ dsRNA. Silencing on transcript level was detected at day 2 and expression further decreased as the flies spent longer on the dsRNA-containing diet, until gene expression was silenced by $79 \%$ at day 6 of the experiment. In all these studies, successful silencing at the transcript level was also linked to clear phenotypical changes in the insects, depending on the gene that was silenced. Despite these successful experiments, Bactrocera, like other dipterans, lacks the sid-1 gene and therefore has to rely solely on the endocytotic pathway for cellular uptake of dsRNA, which might impact its sensitivity for oral RNAi. In fact, a study by Li et al. (2015b) seems to suggest that insects may develop an insensitivity to RNAi caused by feeding of RNAi, through the downregulation of clathrin-mediated endocytosis.

The 31 oral intake RNAi studies on non-fruitfly dipteran insects which we encountered in our literature search, targeted the housefly Musca domestica and important pathogen vectors such as the tsetse fly Glossina morsitans and mosquitoes of the Aedes, Anopheles and Culex genera. Successful RNAi was achieved using siRNA or dsRNA delivered either by soaking, artificial diet or bloodmeal. In G. morsitans adults, Walshe et al. (2009) demonstrated that RNAi could be achieved by mixing dsRNA to bloodmeal. Semi-quantitative PCR indicated a knockdown of around $50 \%$ could be achieved for several days when feeding a $10 \mu \mathrm{g}$ dose of dsRNA. In another study using the same feeding method, knockdown of serpins managed to cause a decrease of trypanosoma infection (Ooi et al., 2015). In M. domestica, an approach mixing dsRNA-producing bacteria was used to achieve up to $76 \%$ gene knockdown (Tang et al., 2012a; Tang et al., 2012b). In mosquitoes, several studies have shown successful RNAi, mostly in larvae. Interestingly, the dsRNA was encapsulated in several of these studies, indicating a less than optimal efficiency when feeding naked dsRNA. Zhang et al. (2010) demonstrated that soaking $A$. gambiae larvae in a chitosan-coated dsRNA solution could lead to efficient RNAi knockdown. In another study, dsRNA was encapsulated by a transfection agent and A. aegypti larvae were placed in a $20 \mathrm{ng} / \mu \mathrm{L}$ solution of this encapsulated dsRNA for 16 hours. This delivery method led to very efficient knockdown (up to $98 \%$, evaluated by semi-quantitative PCR) (Cancino-Rodezno et al., 2010; Cancino-Rodezno et al., 2012). Nonetheless, experiments with naked dsRNA have also been shown to be successful. The Bona et al. (2016) study of silencing the VGSC gene in larval mosquitoes showed that soaking $A$. aegypti larvae in a $0.5 \mu \mathrm{g} / \mu \mathrm{L}$ concentration of dsRNA could lead to a significant silencing effect $( \pm 75 \%)$, and also led to an increase of mortality of about $50 \%$. Lower concentrations that were tested did not elicit a significant gene knockdown. The study also showed that the strong silencing effect was lost as larvae developed into adults. In another study, three different concentrations of dsRNA targeting the NAT5 gene $(0.05,0.5$ and $5 \mu \mathrm{g} / \mu \mathrm{L})$ were tested in $A$. aegypti and, while the lowest concentration did lead to some silencing on day 4, the

The present document has been produced and adopted by the bodies identified above as authors. This task has been carried out exclusively by the author(s) in the context of a contract between the European Food Safety Authority and the authors, awarded following a tender procedure. The present document is published complying with the transparency principle to which the Authority is subject. It may not be considered as an output adopted by the Authority. The European Food Safety Authority reserves its rights, view and position as regards the issues addressed and the conclusions reached in the present document, without prejudice to the rights of the authors. 
other two concentrations led to much higher knockdown levels. Also in terms of phenotypic effect, there was a clear concentration-dependent effect (Meleshkevitch et al., 2013). These and other studies suggest that while soaking or feeding naked dsRNA is able to incite successful gene knockdown, the necessary concentrations are higher and the effect is weaker in comparison to experiments where encapsulated dsRNA was used.

\section{Coleoptera}

Baum et al. (2007) investigated the use of RNAi for protection against coleopteran crop pest insects. In their study, they focused on the WCR, D. virgifera, and the CPB, L. decemlineata. These two species were known to be among the most sensitive insects to RNAi, with $50 \%$ lethal concentration $\left(\mathrm{LC}_{50}\right)$ values in the $1-10 \mathrm{ppb}(1-10 \mathrm{pg} / \mu \mathrm{L})$ range (Baum and Roberts, 2014), which is lower than anything observed in other insect orders. Furthermore, some other coleopteran species, such as the canola flea beetle Phyllotreta striolata (Zhao et al., 2008) seem to show similar efficiency. However, even within Coleoptera there is variability in terms of RNAi sensitivity. In T. castaneum for example, dsRNA delivered through flour discs at a concentration of $100 \mathrm{ng} / \mathrm{mg}$ diet was necessary to achieve a significant silencing of the $\mathrm{Na}_{v}$ gene (Abd El Halim et al., 2016) and Whyard et al. (2009) reported an $\mathrm{LC}_{50}$ of $3.3 \mu \mathrm{g} / \mathrm{mg}$ of dsRNA targeting V-ATPase. Recently, two studies on the African sweet potato weevils Cylas puncticollis and Cylas brunneus demonstrated that even between two very closely related species, a significant amount of variability in RNAi sensitivity could be observed, with C. brunneus being much more sensitive to oral RNAi than $C$. puncticollis, which was rather recalcitrant to oral RNAi using naked dsRNA (Christiaens et al., 2016; Prentice et al., 2017). In C. brunneus, feeding on diet containing $1 \mu \mathrm{g} / \mathrm{mL}$ dsRNA led to $67 \%-73 \%$ gene knockdown, while in C. puncticollis, a concentration of $30 \mu \mathrm{g} / \mu \mathrm{L}$ was necessary to achieve similar knockdown levels. The authors also found the variable sensitivity to be related to differences in dsRNA stability in the gut of these two weevils. In most of the oral RNAi studies retrieved in our search, the dsRNA was mixed with artificial diet, coated on leaves or presented through GM plant material. However, a study of the banana root borer Cosmopolites sordidus demonstrated that a topical application of 200-2000 ng of dsRNA targeting the ubiquitinE2 gene on the eggs also elicited successful gene silencing in hatching larvae, eventually causing $100 \%$ mortality 21 days after hatching (Ocimati et al., 2016).

\section{Lepidoptera}

Lepidoptera comprise some of the most devastating agricultural pests and have therefore received a lot of attention from researchers investigating whether RNAi could be an effective pest control strategy. Experiments using microinjection in some of these lepidopteran species had already indicated that the RNAi pathway is present and functional, and that relatively small amounts of dsRNA were necessary for efficient gene knockdown (Bettencourt et al., 2002; Hirai et al., 2004; Terenius et al., 2007). However, when attempting feeding dsRNA to these insects, it became apparent that the sensitivity to oral RNAi in Lepidoptera was generally quite low, and very variable at best in comparison with Coleoptera. A meta-study by Terenius et al. (2011) gathered all data available on RNAi in Lepidoptera, both published and unpublished studies and concluded that while oral RNAi sometimes works in some species at very high doses of dsRNA, these species are generally refractory to feeding RNAi. They evaluated a total of 32 lepidopteran RNAi studies and reported low efficiency for 5 of those and a complete lack of successful silencing in 18 studies. An efficient silencing response was only observed in nine out of 32 studies.

Manduca sexta was one of the species generally showing moderate levels of oral RNAi efficiency. We retrieved eight studies in total on $M$. sexta RNAi, most of them delivering the dsRNA through feeding of a droplet of water containing 4-5 $\mu \mathrm{g}$ dsRNA to caterpillars (Cancino-Rodezno et al., 2010; FloresEscobar et al., 2013; Gomez et al., 2015; Porta et al., 2011). Three studies also successfully targeted M. sexta larvae through Nicotiana attenuata plant-expression of dsRNA which elicited an efficient gene knockdown (Kumar et al., 2012; Kumar et al., 2014; Poreddy et al., 2015). The majority of the lepidopteran oral RNAi studies we retrieved were targeting $H$. armigera, even though the data from Terenius et al. (2011) suggested that oral RNAi in this species is rather unsuccessful. In most

The present document has been produced and adopted by the bodies identified above as authors. This task has been carried out exclusively by the author(s) in the context of a contract between the European Food Safety Authority and the authors, awarded following a tender procedure. The present document is published complying with the transparency principle to which the Authority is subject. It may not be considered as an output adopted by the Authority. The European Food Safety Authority reserves its rights, view and position as regards the issues addressed and the conclusions reached in the present document, without prejudice to the rights of the authors. 
$H$. armigera studies, concentrations in the $1-10 \mu \mathrm{g} / \mu \mathrm{L}$ range were used. However, one study also reported successful gene knockdown after feeding $35 \mathrm{ng}$ dsRNA/ $\mu \mathrm{g}$ artificial diet to second-instars, leading to near complete knockdown after 24 hours. Gene expression in this study started to recover after 48 hours (Zhang et al., 2015d). Interestingly, the potential for insecticidal miRNAs was also explored in $H$. armigera. Tomato plants were genetically modified to express an artificial miRNA-24 specifically silencing the chitinase gene in $H$. armigera, resulting in successful silencing and toxic effects similar to those seen when using dsRNA to silence chitinase (Agrawal et al., 2015). Other species for which successful RNAi was reported were Heliothis virescens, Ostrinia sp., P. xylostella, Chilo sp. and several Spodoptera species (Apone et al., 2014; Ayra-Pardo et al., 2015; Chen et al., 2014b; Khajuria et al., 2010; Qiu et al., 2015; Zhang et al., 2012; Zhu et al., 2016). Despite these successful experiments, often requiring high doses, there is a consensus that oral RNAi in Lepidoptera does not work very efficiently. Two possible explanations have been suggested, namely a very strong nucleolytic degradation of dsRNA in the digestive system of these caterpillars, and potential issues with cellular uptake or cytoplasmic release (Arimatsu et al., 2007; Garbutt et al., 2013; Liu et al., 2012; Shukla et al., 2016). These factors are discussed further in Section 5.3.3.

\section{Hymenoptera}

Hymenopteran species are an interesting order, since they comprise economically important species such as pollinators and parasitic wasps. Most studies we retrieved on hymenopteran RNAi reported experiments on the honeybee, $A$. mellifera. Several studies explore the potential use of RNAi as a therapeutic, targeting honeybee-specific viruses and also the Varroa mite ( $V$. destructor). Feeding a $1 \mu \mathrm{g}$ dose of Israeli Acute Paralysis Virus (IAPV) specific siRNA mixed with sugar water was able to inhibit viral replication in the honeybees, demonstrating that the technology could indeed be used for therapeutic purposes (Chen et al., 2014c; Hunter et al., 2010; Maori et al., 2009). Similarly, feeding of $1 \mu \mathrm{g}$ deformed wing virus (DWV)-specific dsRNA every day for six days led to significantly lower viral titres upon infection (Desai et al., 2012). Maori et al. (2012) also managed to decrease $V$. destructor incidence by $60 \%$ after feeding parasitized honeybees with a $40 \mu \mathrm{g} / \mu \mathrm{L} V$. destructor specific dsRNA sugar water solution. Successful RNAi experiments targeting honeybee genes were also reported. Nunes and Simões (2009) reported around 90\% silencing of the vitellogenin gene expression upon feeding of $0.5 \mu \mathrm{g}$ dsRNA to honeybee larva.

Data in other Hymenoptera is scarce. One study showed that RNAi in the bumblebee Bombus terrestris, which is an important commercial pollinator, can be used efficiently to target IAPV virus infections in the bumblebee, using high doses of $2 \mu \mathrm{g}$ dsRNA per day per individual (Piot et al., 2015). No studies targeting Bombus-specific genes by feeding dsRNA were found. Injection studies however reveal that even with that delivery method, high doses of $20 \mu \mathrm{g}$ were necessary to elicit a silencing response of an endogenous $B$. terrestris gene (Niu et al., 2016). In another study, targeting the wasp Polistes metricus, very high doses of dsRNA ( $80 \mu \mathrm{g}$ dsRNA over two days) resulted in only a modest silencing effect. It must be noted, however, that only one timepoint was chosen for sampling (Hunt et al., 2011). Finally, targeting three genes in the ant Camponotus floridanus, fed ad libitum on $2 \mu \mathrm{g}$ dsRNA/ $\mu \mathrm{L}$ diet, resulted in an observable silencing effect for only one of the two targeted genes in the body of minor workers, suggesting $C$. floridanus is not extremely sensitive to oral RNAi compared to other more sensitive insect species (Ratzka et al., 2013). Based on these data, RNAi efficiency in Hymenoptera in general does not seem to work highly efficiently. Of course, one has to take into account the size and weight of many of the hymenopteran species studied in these researches, such as honeybees and bumblebees, but, even taking this into account, the efficiency in certain Coleoptera and Hemiptera is considerably higher.

\section{Hemiptera}

The order of Hemiptera also comprises many pest insects, including whiteflies, aphids, stinkbugs, planthoppers and thrips. In many of these species, RNAi by oral feeding has been shown to be moderately efficient, albeit variable in some species (Christiaens and Smagghe, 2014). In the pea aphid, A. pisum, several studies report successful RNAi at concentrations ranging from $750 \mathrm{ng} / \mu \mathrm{L}$ to

The present document has been produced and adopted by the bodies identified above as authors. This task has been carried out exclusively by the author(s) in the context of a contract between the European Food Safety Authority and the authors, awarded following a tender procedure. The present document is published complying with the transparency principle to which the Authority is subject. It may not be considered as an output adopted by the Authority. The European Food Safety Authority reserves its rights, view and position as regards the issues addressed and the conclusions reached in the present document, without prejudice to the rights of the authors. 
$5 \mu \mathrm{g} / \mu \mathrm{L}$ (Mao and Zeng, 2012; Sapountzis et al., 2014; Shakesby et al., 2009; Whyard et al., 2009). However, the knockdown levels and phenotypical effects were not always that dramatic. For example, Mao and Zeng (2012) targeted hunchback in A. pisum by placing aphids on a $750 \mathrm{ng} / \mu \mathrm{L}$ dsRNA containing artificial diet. The highest transcript silencing was recorded at day seven, with $46 \%$ silencing. In terms of mortality, $45 \%$ of dsRNA-treated aphids were dead on day seven, while this was $20 \%$ in the control. In another study, Shakesby et al. (2009) targeted an aquaporin gene using a continuous feeding approach on a $1.8 \mu \mathrm{g} / \mu \mathrm{L}$ dsRNA containing artificial diet. After six days on this diet, the recorded mortality was $46 \%$, compared to $19 \%$ in the GFP control. These authors observed significant silencing in at least one tissue for 12 aphids of the 15 aphids they analysed. In contrast, another study reported a failure to elicit efficient RNAi in $A$. pisum, both with feeding and injection and suggested possible strain-related variabilities in RNAi sensitivity (Christiaens and Smagghe, 2014; Christiaens et al., 2014). In A. pisum, degradation of dsRNA by saliva and in haemolymph was also reported, similar to observations in the tarnished plant bug Lygus lineolaris (Allen and Walker, 2012; Christiaens et al., 2014). Two other aphids, the green peach aphid M. persicae and the wheat aphid Sitobion avenae have been extensively studied and efficient oral RNAi was reported in both species. In $S$. avenae, very low doses of dsRNA, as low as 3-20 $\mathrm{ng}$ dsRNA/ $\mu \mathrm{L}$ diet were sufficient to elicit successful gene knockdown (Deng and Zhao, 2014; Fan et al., 2015; Zhang et al., 2013; Zhang et al., 2016). In M. persicae, all studies which were retrieved via the systematic literature search used GM plants to elicit RNAi silencing. Target gene silencing was confirmed in aphids feeding on these transgenic plants both at the transcript level and based on phenotypical observations such as mortality, decrease in fecundity, etc. (Bhatia et al., 2012; Coleman et al., 2014; Elzinga et al., 2014; Pitino et al., 2011; Pitino and Hogenhout, 2013). Interestingly, one study also included a miRNA construct, targeting acetylcholine esterase in M. persicae. They observed similar results for the miRNA expressing plants compared to those expressing hpRNAs targeting the same gene. However gene knockdown levels did not exceed 30\% (Guo et al., 2014). Succesful oral RNAi has also been reported in Bemisia tabaci whiteflies. Most studies exposed the insects to concentrations around $200 \mathrm{ng} / \mu \mathrm{L}$ and $2 \mu \mathrm{g} / \mu \mathrm{L}$ to achieve successful RNAi. Two studies however used a range of concentrations, including some which were considerably lower. Shim et al. (2015) found that $2.5 \mathrm{ng} / \mu \mathrm{L}$ dsRNA, targeting hsp70, did not cause any observable silencing at the transcript level, while $25 \mathrm{ng} / \mu \mathrm{L}$ ( $66 \%$ silencing) and $250 \mathrm{ng} / \mu \mathrm{L}$ (near complete knockdown) did cause significant silencing. Phenotypically, feeding on $250 \mathrm{ng} / \mu \mathrm{L}$ dsRNA for 24 hours led to approximately $40 \%$ mortality at day six post treatment, while continuous feeding of dsRNA resulted in approximately $80 \%$ mortality after six days. Upadhyay et al. (2011) performed extensive research on the efficiency of RNAi in whiteflies, testing the difference between dsRNA and siRNA, testing different target genes and a range of concentrations. They found that dsRNA was only slightly more efficient than siRNA and reported an $\mathrm{LC}_{50}$ value for dsRNA targeting V-ATPase of $3.08 \mathrm{ng} / \mu \mathrm{L}$, indicating that oral RNAi in whiteflies is highly efficient. In the brown planthopper, $N$. lugens, most studies report dsRNA concentrations in artificial diet of 100-1000 $\mathrm{ng} / \mu \mathrm{L}$ dsRNA. Hao et al. (2015) investigated different concentrations and found that while concentrations as low as $20 \mathrm{ng} / \mu \mathrm{L}$ could elicit significant knockdown after seven days, the effect was limited and concentrations of $100-500 \mathrm{ng} / \mu \mathrm{L}$ were necessary to elicit efficient knockdown leading to an observable phenotype. These results confirmed earlier observations by Chen et al. (2010) who reported that $500 \mathrm{ng} / \mu \mathrm{L}$ dsRNA targeting trehalose phosphate synthase was necessary to cause phenotypic effects, while $20 \mathrm{ng} / \mu \mathrm{L}$ and $100 \mathrm{ng} / \mu \mathrm{L}$ did not cause any observable effects. Thus, these studies indicate that higher concentrations seem to be necessary for successful RNAi silencing in brown planthopper.

\section{Orthoptera}

Orthoptera are an interesting group of insects in terms of RNAi efficiency. Several publications have reported different locust species such as $S$. gregaria and L. migratoria to be highly sensitive to RNAi by microinjection, while feeding dsRNA, even very high doses, does not result in successful gene knockdown (Luo et al., 2013; Wynant et al., 2014b; Wynant et al., 2012). The possible mechanisms behind these observations will be discussed further on in this Technical Report.

The present document has been produced and adopted by the bodies identified above as authors. This task has been carried out exclusively by the author(s) in the context of a contract between the European Food Safety Authority and the authors, awarded following a tender procedure. The present document is published complying with the transparency principle to which the Authority is subject. It may not be considered as an output adopted by the Authority. The European Food Safety Authority reserves its rights, view and position as regards the issues addressed and the conclusions reached in the present document, without prejudice to the rights of the authors. 


\section{Blattodea}

In Blattodea, no oral RNAi has been successfully achieved, to the best of our knowledge. However, RNAi by injection in the cockroach B. germanica works efficiently (Cruz et al., 2006; Garbutt et al., 2013).

\section{Conclusions on Insecta}

Only a relatively small number of studies examine non-target insects, or even beneficial insects, exposed to dsRNA orally. Nevertheless, the insects for which data are available represent all major insect orders, giving us an idea of the range of RNAi efficiency and sensitivity in insects. Indeed, in general terms, the data seem to indicate that there are some inter-order differences in RNAi sensitivity. Generally, many Coleoptera seem to be very sensitive to oral RNAi while Lepidoptera are not. In Hemiptera and Diptera, the sensitivity seems rather variable between species while in orthoptera RNAi by injection seems to work highly efficiently, while locusts appear to be highly refractory to oral RNAi.

\subsubsection{Crustacea}

In our systematic literature search, we identified 21 studies reporting oral RNAi in Crustacea. The majority were studies on decapod Penaeus species. There is great interest in using RNAi to combat viral infections in these shrimps, which are economically important commodities in South-East Asia. Therefore, most Penaeus oral RNAi studies targeted viruses infecting these shrimps. In 2013, researchers used a bacterial approach to deliver dsRNA, specific for the Laem Singh virus (LSNV), to P. monodon shrimp. In these dsRNA treated shrimp, a reduction of 20-60 \% LSNV was observed (Saksmerprome et al., 2013). Other studies also confirmed the functionality of orally delivered dsRNA expressing bacteria, as an antiviral therapeutic in P. monodon, Penaeus merguiensis and Penaeus vannamei (Leigh et al., 2015; Saksmerprome et al., 2013; Sanitt et al., 2016; Sanitt et al., 2014; Sarathi et al., 2008; Sarathi et al., 2010). However, we found one study in which Penaeus-specific genes were targeted. Here, feeding of bacteria expressing dsRNA specific for the Rab7 and Stat genes in $P$. monodon and $L$. vannamei caused successful silencing in both hepatopancreas and gill tissues (Attasart et al., 2013). In most of these studies, $E$. coli was used to deliver the dsRNA. However, two studies reported using nanoparticle-coated dsRNA. Sanitt et al. (2016) reported the use of cholesterol-based liposomic particles to increase RNAi efficiency against the yellow head virus (YHV) in $P$. vannamei. Another study reported the successful use of a chitosan-dextran sulfate nanocapsule to delivery dsRNA in P. monodon post-larvae (Ramesh Kumar et al., 2016).

Nanoparticle-coated dsRNA was also used in the copepod Tigriopus californicus (Barreto et al., 2015). However, the two carriers that were tested, chitosan polymer and a liposome transfection agent, did not result in successful RNAi silencing (Barreto et al., 2015). In the salmon louse, Lepeophteirus salmonis, researchers managed to elicit RNAi knockdown by soaking larvae in a $10 \mathrm{ng} / \mu \mathrm{L}$ dsRNA solution. However, RNAi was only successful when animals were treated at the time they moulted from Nauplius I to Nauplius II stages (Eichner et al., 2015a; Eichner et al., 2014; Eichner et al., 2015b). In the waterfleas Daphnia pulex, Daphnia melanica and Daphnia pulicaria, successful RNAi was achieved by adding bacteria expressing Daphnia-specific dsRNA to the aquatic medium containing the animals. Semi-quantitative transcript analysis, as well as phenotypical observations, suggest an efficient knockdown using this method (Schumpert et al., 2016; Schumpert et al., 2015). Finally, one study also reported that soaking larvae and nauplii of the barnacle Amphibalanus amphitrite in $0.8 \mathrm{~nL} / \mu \mathrm{L}$ siRNA concentration, using a liposomic transfection agent, was successful (Zhang et al., 2015a).

In conclusion, the amount of data on RNAi efficiency in Crustacea is rather limited, and most studies report successful RNAi in the decapod shrimps of the Penaeus genus using either bacteria or nanocarrier-coated dsRNA for virus control. The lack of studies using naked dsRNA may reflect a refractoriness in these shrimp species, or may be related to the short half-life of these dsRNA

The present document has been produced and adopted by the bodies identified above as authors. This task has been carried out exclusively by the author(s) in the context of a contract between the European Food Safety Authority and the authors, awarded following a tender procedure. The present document is published complying with the transparency principle to which the Authority is subject. It may not be considered as an output adopted by the Authority. The European Food Safety Authority reserves its rights, view and position as regards the issues addressed and the conclusions reached in the present document, without prejudice to the rights of the authors. 
molecules in the environment. In other Crustacea, the sensitivity to RNAi proved to be variable using naked dsRNA or siRNA.

\subsubsection{Chelicerata}

In our systematic literature study, we found reports that mites and ticks were susceptible to oral RNAi but no studies on species outside of the Acari subclass were found in the context of oral RNAi. One of the first demonstrations of successful feeding RNAi was delivered by Soares et al. (2005), who managed to elicit successful RNAi knockdown in I. scapularis tick nymphs. In this study, a capillary feeding approach was used, eventually delivering a dose of $2.4 \mu \mathrm{g}$ dsRNA to each nymph. Northern blot analysis confirmed successful silencing of the isac gene and observed effects included a $40.6 \%$ reduction in weight. Sensitivity to oral RNAi was also demonstrated in another ixodid tick, namely Haemaphysalis longicornis (Galay et al., 2016). Immersion of $H$. longicornis ticks in a $1 \mu \mathrm{g} / \mu \mathrm{L}$ dsRNA containing solution led to efficient knockdown in all stages (Galay et al., 2016; Gong et al., 2009).

Also in mites, feeding RNAi seems to be functional. We identified four studies reporting successful gene knockdown in Tetranychus species. Two different delivery methods have been used in T. urticae. One entails a leaf-disk mediated delivery, whereby mites are placed on dsRNA-permeated leaf disks in a feeding apparatus. In one study, this delivery led to only $26 \%$ knockdown of the target gene COPE after 14 hours, and $14.6 \%$ knockdown after 72 hours. In another study, targeting other genes, the researchers managed to achieve $50 \%$ silencing for V-ATPase after 48 hours. For other genes, knockdown efficiency was lower (Kwon et al., 2016; Kwon et al., 2013). The same delivery method was used in Tetranychus cinnabarinus, leading to up to 64\% knockdown efficiency (Shi et al., 2016). Another delivery method, which was used in T. urticae, was feeding of dsRNA through an impregnated piece of cotton, leading to a $42 \%$ transcript knockdown after three days feeding on the dsRNA (Ozawa et al., 2012). In another Acari species, feeding RNAi to the predatory mite Metaseiulus occidentalis also elicited a silencing response. In these studies, delivery of dsRNA was achieved by placing mites on a parafilm disc resting on a water-soaked piece of cotton containing a $350 \mathrm{ng} / \mu \mathrm{L}$ dsRNA (Pomerantz and Hoy, 2015; Wu and Hoy, 2014a; Wu and Hoy, 2014b; Wu and Hoy, 2015). Reported expression knockdown in these experiments was between $70 \%$ and $91 \%$, confirming efficient silencing. Also in other mites such as the house dust mite, Dermatophagoides pteronyssinus, the citrus red spider mite, Panonychus citri, and the red poultry mite, Dermanyssus gallinae, RNAi by feeding dsRNA was shown to be functional (Kamau et al., 2013; Marr et al., 2015; Tian et al., 2015b).

The present document has been produced and adopted by the bodies identified above as authors. This task has been carried out exclusively by the author(s) in the context of a contract between the European Food Safety Authority and the authors, awarded following a tender procedure. The present document is published complying with the transparency principle to which the Authority is subject. It may not be considered as an output adopted by the Authority. The European Food Safety Authority reserves its rights, view and position as regards the issues addressed and the conclusions reached in the present document, without prejudice to the rights of the authors. 
Table 12: Overview of reported sRNA concentrations or single doses leading to successful RNAi-elicited gene silencing in in planta, in vitro feeding and soaking studies in Arthropoda

\begin{tabular}{|c|c|c|c|c|c|c|}
\hline Subphylum & Order & In planta $\mathbf{G M}^{1}$ & $\begin{array}{l}\text { In planta } \\
\text { non-GM }\end{array}$ & In vitro feeding & $\begin{array}{l}\text { In vitro } \\
\text { soaking }\end{array}$ & Comments \\
\hline \multirow[t]{7}{*}{ Hexapoda } & Diptera & - & - & $0.02-6 \mu \mathrm{g} / \mu \mathrm{L}$ & $\begin{array}{l}0.05-0.5 \\
\mu g / \mu L\end{array}$ & $\begin{array}{l}\text { Diptera are sensitive to RNAi by injection of naked dsRNA or } \\
\text { siRNA, but require formulation with a polymer or liposome for } \\
\text { feeding RNAi }\end{array}$ \\
\hline & Lepidoptera & Sni & $1-3 \mu \mathrm{g} / \mathrm{cm}^{2}$ & $0.015-10 \mu \mathrm{g} / \mu \mathrm{L}$ & - & $\begin{array}{l}\text { Additional delivery methods, such as forcefeeding single doses } \\
\text { (usually several } \mu \mathrm{g} \text { ) have been reported }\end{array}$ \\
\hline & Coleoptera & $\begin{array}{c}50 \mathrm{E}-6-0.5 \\
\mu \mathrm{gg} / \mu \mathrm{L} \\
0.5-16 \mathrm{ng} / \mathrm{cm}^{2}\end{array}$ & Sni & $1 \mathrm{E}-6-5 \mu \mathrm{g} / \mu \mathrm{L}$ & - & $\begin{array}{l}\text { Very high sensitivity by feeding, compared to other insect } \\
\text { orders, although some variability exists between different } \\
\text { coleopteran species }\end{array}$ \\
\hline & Hymenoptera & - & - & $\begin{array}{l}0.02-2.2 \mu \mathrm{g} / \mu \mathrm{L} \\
1-40 \mu \mathrm{g} \text { SD per day }\end{array}$ & - & \\
\hline & Hemiptera & Sni & Sni & $0.003-40 \mu \mathrm{g} / \mu \mathrm{L}$ & - & \\
\hline & Orthoptera & - & - & $\begin{array}{l}3 \mu \mathrm{g} / \mu \mathrm{L} \\
6 \mu \mathrm{g} \mathrm{SD} \text { per day }\end{array}$ & - & $\begin{array}{l}\text { In Schistocerca, a dose of } 1 \mathrm{mg} \text { per day for eight days could } \\
\text { not trigger any RNAi knockdown }\end{array}$ \\
\hline & Blattodea & - & - & $\begin{array}{l}0.15 \mu \mathrm{g} / \mu \mathrm{L} \\
2.2-5.1 \mathrm{mg} / \mathrm{cm}^{2} \\
10 \mu \mathrm{g} \mathrm{SD}\end{array}$ & - & \\
\hline Crustacea & & - & - & $6-12 \mathrm{mg} / \mathrm{kg}$ & $\begin{array}{c}0.01-1 \\
\mu \mathrm{g} / \mu \mathrm{L}\end{array}$ & $\begin{array}{l}\text { E. coli- or Artemia-mediated delivery, or a polymer-formulation } \\
\text { used in most Crustacea feeding studies }\end{array}$ \\
\hline Chelicerata & & - & Sni & $0.02-1.2 \mu \mathrm{g} / \mu \mathrm{L}$ & - & \\
\hline
\end{tabular}

Sni: Succesful silencing but no information on concentration or expression level in the plant; SD: single dose

${ }^{1}$ Concentrations for in planta studies were most often not given; ${ }^{2}$ These include leaf immersion, stem injection, root absorption delivery strategies

- : No studies found

The present document has been produced and adopted by the bodies identified above as authors. This task has been carried out exclusively by the author(s) in the context of a contract between the European Food Safety Authority and the authors, awarded following a tender procedure. The present document is published complying with the transparency principle to which the Authority is subject. It may not be considered as an output adopted by the Authority. The European Food Safety Authority reserves its rights, view and position as regards the issues addressed and the conclusions reached in the present document, without prejudice to the rights of the authors. 


\subsubsection{Annelids and molluscs}

Very little information is available concerning RNAi sensitivity for both annelids and molluscs. In both classes there are a number studies demonstrating that injection of dsRNA or morpholinos can be used to elicit gene knockdown (e.g., Conzelmann et al., 2013; Song et al., 2002), but only three studies were retrieved demonstrating a soaking dsRNA approach in molluscs (Chen et al., 2014a; Knight et al., 2011; Wang et al., 2016a), and no studies of direct feeding were found. In one study, solutions ranging between 0.1 and $10 \mu \mathrm{g} d s R N A / m L$ were used to target the Asiatic hard clam, Meretrix meretrix, and while knockdown efficiencies were not investigated at the transcript level, a near $100 \%$ mortality was observed in the RNAi-treated clam larvae, including at the $0.1 \mathrm{ng} / \mu \mathrm{L}$ concentration. This suggests that RNAi by soaking works efficiently in this species. Another study, investigating the role of Prx and CathB genes in schistosomiasis transmission by the freshwater snail Biomphalaria glabrata, demonstrated that soaking in a siRNA or dsRNA solution only resulted in silencing when the sRNAs were coated with the polyethyleneimine (PEI) polymer. Naked dsRNA or siRNA did not result in an observable knockdown (Knight et al., 2011), suggesting that the stability of sRNAs might be compromised in this animal, or in this experimental setup. Finally, in the abalone Haliothis diversicolor, efficient knockdown was reported using a $5 \mathrm{ng} / \mu \mathrm{L}$ soaking solution (Wang et al., 2016a). The concentrations used in some of these studies suggest that at least some molluscs are very sensitive to RNAi.

\subsubsection{Factors involved in oral RNAi efficiency}

In this section, we discuss different biotic, abiotic, biochemical and physiological factors that can influence RNAi efficacy in invertebrates. Many studies have been devoted to the variability in RNAi efficiency in invertebrates, notably in insects. While some factors, such as the stability of RNAs in the body and their cellular uptake, are thoroughly investigated in a range of species, others are still poorly understood, such as the effect of viral infections.

\subsubsection{RNAi machinery genes repertoire}

RNAi efficiency is very much dependent on the presence of the RNAi (core) machinery genes in the genome. Despite the fact that RNAi is a conserved mechanism throughout Eukaryota, considerable differences in pathways and functioning proteins in these pathways exist between the different taxonomical clades. For example, while plants possess four Dicer-like (DCL) protein encoding genes in their genomes, insects only have two, and nematodes, annelids, molluscs and higher animals have only one (Mukherjee et al., 2013). Regarding Argonaute-related proteins, there is even more diversity to be found. For example, while most insects have five Ago-like proteins (Ago1, Ago2, Ago3, PIWI and Aubergine), eight Ago-like proteins have been found in humans, 10 in the plant Arabidopsis thaliana and 27 in nematodes (Höck and Meister, 2008). While not all of these Ago-like proteins have functions in the RNAi pathways, many do. And this diversity of core gene repertoire is also reflected in the diversity and compartmentalization of the different RNAi-related functions in eukaryotic cells. In insects for example, siRNAs, miRNAs and piRNAs are mainly processed by distinct pathways using different dicer and Ago-like proteins. While in mammals, all sRNAs are processed by the one Dicer that is present. Since further comparisons of these RNAi pathways in non-invertebrates falls outside of the scope of this review, we can refer to some excellent recent research articles and reviews for further reading on the diversity of RNAi pathways in Eukaryota (Chapman and Carrington, 2007; Ding and Voinnet, 2007; Höck and Meister, 2008; Mukherjee et al., 2013; Obbard et al., 2009; Pačes et al., 2017).

So far, there is no report on cell-autonomous RNAi not being functional in any invertebrate, to the best of our knowledge. Given the important functions RNAi is involved in, this is no surprise. This also means that in terms of core genes involved in intracellular RNAi, such as Dicer and Ago, we should not expect important qualitative differences between species. Nonetheless, it has been reported that

The present document has been produced and adopted by the bodies identified above as authors. This task has been carried out exclusively by the author(s) in the context of a contract between the European Food Safety Authority and the authors, awarded following a tender procedure. The present document is published complying with the transparency principle to which the Authority is subject. It may not be considered as an output adopted by the Authority. The European Food Safety Authority reserves its rights, view and position as regards the issues addressed and the conclusions reached in the present document, without prejudice to the rights of the authors. 
RNAi-related genes belong to some of the most rapidly evolving genes, similar to other immunity pathway genes (Obbard et al., 2006), so differences do exist and will be discussed here, including whether they could have an impact on RNAi efficiency.

As discussed before, despite $C$. elegans being very sensitive to (oral) RNAi, other nematodes such as parasitic species are often more refractory. One of the possible reasons is a difference in RNAi-related machinery genes repertoire. A study looking into the RNAi-pathway related genes in a wide range of nematodes showed that Caenorhabditis species have an expanded RNAi-related gene repertoire compared to parasitic nematodes, especially concerning Ago genes and the genes related to uptake and spread of the RNAi signal (Dalzell et al., 2011). The variability in effector proteins involved in uptake and systemic spread was discussed in detail in Section 5.1. However, differences can also be found in other pathways, such as genes involved in small RNA biosynthesis, RISC elements and genes involved in amplification and secondary siRNA production. For example, proteins involved in the biosynthesis of small RNAs, such as RNase III enzymes (Drosha, Pasha, Dicer) were found to be broadly conserved, as were RNA helicases and exportins. However, orthologs of the dicer-cofactor RDE-4 were found to be absent in most parasitic nematodes. Another interesting find was that Ago genes involved in internal gene regulation were broadly conserved throughout nematodes, but this was not the case for Ago genes involved in RNAi induced by exogenous RNAi (Dalzell et al., 2011). C. elegans possesses at least 27 distinct Ago proteins. These Ago proteins, by being an important part of the RISC complex, are involved in different RNAi-related pathways. Different subsets could be identified such as those involved in miRNA processing (e.g., ALG-1), those that interact with endosiRNA (e.g., ALG-4 and WAGO's) and the Ago that is responsible for RNAi triggered by exogenous dsRNA (RDE-1). While the former Ago's were found to be broadly conserved throughout nematodes, this was not the case for RDE- 1 which was not found in plant-parasitic nematodes. However, the authors note that their identification strategy, which was based on BLAST analysis of known C. elegans RNAi effectors, could not account for the presence of other uncharacterized Ago's which could have assumed the role of those $C$. elegans Ago's that were not identified. Alternatively, other Ago's which were identified in these species could have assumed other roles than they have in C. elegans (Dalzell et al., 2011). In regard to the amplification of the RNAi signal through RDRPdependent secondary siRNA production, most nematodes appear to have at least some of the RdRPs that are implicated in this mechanism, such as smg-2, smg- 6 and ego-1. In contrast, smg-5 and rsd-2 seem to be only present in Caenorhabditis genomes. However, given the fact that in a number of plant parasitic nematodes efficient RNAi after exposure to very small doses has been observed, the absence of these RDRPs does not seem to affect RNAi efficiency in these species (Dalzell et al., 2010a; Dalzell et al., 2011). One important conclusion the authors made was that a clear link between the RNAi efficiency and the absence of certain effector genes in the investigated nematode species could not be made. In fact, while Caenorhabditis species, and especially $C$. elegans, do have an expanded gene repertoire, the difference is mainly quantitative rather than qualitative. All distinct pathways and essential proteins are represented throughout the nematode class (Dalzell et al., 2011).

For a long time, information on the RNAi core machinery in arthropods was limited to the model insect $D$. melanogaster. However, recently a number of in silico studies have been devoted to the RNAi-related gene repertoire in other arthropod species, including the Colorado potato beetle L. decemlineata (Swevers et al., 2013b), the red flour beetle T. castaneum (Tomoyasu et al., 2008), the common North Sea shrimp Crangon crangon (Christiaens et al., 2015), the Asian citrus psyllid D. citri (Taning et al., 2016a) and the soybean aphid Aphis glycines (Bansal and Michel, 2013). In 2016, a large scale study was published investigating 10 RNAi core genes in 100 insect species (Dowling et al., 2016). These included: the miRNA pathway elements Dcr-1, Ago-1, Loqs, Drosha and Pasha; the siRNA pathway components Dcr-2, Ago-2 and R2D2; the piRNA pathway elements Ago3 and Aub/Piwi; and sid-1-like genes. However, since transcriptomic data was used in the study, it is impossible to claim with any certainty that a certain gene is missing in a species. Nevertheless, the study did deliver a number of interesting results. In 2017, a similar study was published looking at the

The present document has been produced and adopted by the bodies identified above as authors. This task has been carried out exclusively by the author(s) in the context of a contract between the European Food Safety Authority and the authors, awarded following a tender procedure. The present document is published complying with the transparency principle to which the Authority is subject. It may not be considered as an output adopted by the Authority. The European Food Safety Authority reserves its rights, view and position as regards the issues addressed and the conclusions reached in the present document, without prejudice to the rights of the authors. 
RNAi core gene repertoire related to antiviral innate immunity in available Crustacea genomes (Lai and Aboobaker, 2017).

Looking at these arthropods, it is clear that the three RNAi pathways are present in all major lineages throughout both subphyla (Dowling et al., 2016), which is not surprising given their roles in antiviral defence, protection of the genome and internal gene regulation. However, some interesting differences could be found between different groups. For example, all insects possess two Dicer-like proteins, while this number varies in some Crustacea and Chelicerata. In the Crustacean Daphnia magna, three Dicer-like proteins were identified, for the first time in animal species (McTaggart et al., 2009). This extra Dicer is the result of a lineage-specific duplication of the Dicer that is involved in the antiviral immune pathway in arthropods, the siRNA pathway, suggesting that it might have an influence on the efficiency of siRNA RNAi as well. However, no further studies have been conducted to elucidate the role of this extra Dicer protein. Furthermore, in other crustacean species in which the RNAi pathways were investigated, such as the brown shrimp C. crangon, the black tiger shrimp P. monodon and the Pacific white shrimp L. vannamei, only two Dicers were found (Chen et al., 2011a; Christiaens et al., 2015; Li et al., 2013; Su et al., 2008; Yao et al., 2010). In ticks and mites (Acari), a variable number of Dicers was reported as well. The first Acari species to have its genome sequenced and annotated, the two-spotted spider mite T. urticae (Grbić et al., 2011), contains one gene copy for Dicer-1 and Dicer-2, as in insects (Suzuki et al., 2017). However, in the ixodid tick I. scapularis, only one Dicer, homologous to Dicer-1, was identified (Kurscheid et al., 2009). Furthermore, five copies of the Dicer-2 gene were identified in the genome of the predatory mite $M$. occidentalis (Hoy et al., 2016). While these differences in Dicer-encoding genes might influence the efficiency of the pathways involved, no actual proof of their effect on RNAi has been reported.

A number of these studies also shed more light on the issue surrounding R2D2. In Drosophila, R2D2 is an important cofactor of Dicer-2 and is required for successful RNAi and a successful antiviral response (Liu et al., 2003; Wang et al., 2006). In many Coleoptera, including T. castaneum, two distinct R2D2 genes were discovered (Dowling et al., 2016; Tomoyasu et al., 2008) while in several Lepidoptera, expression of R2D2 is either very low or the gene is absent (Dowling et al., 2016; Swevers et al., 2011). Furthermore, R2D2 has been reported absent in a number of other arthropod species, such as the insect $D$. citri and the crustacean $C$. crangon (Christiaens et al., 2015; Taning et al., 2016a). Dowling et al. (2016) confirmed that R2D2 was not present in the primary wingless insects they investigated and was also missing in their outgroup species, the crustacean $D$. pulex and the chelicerate I. scapularis. They also report that, to date, R2D2 has not been found outside of the class of Insecta. The authors speculate that Loquacious, a similar protein normally associated to the miRNA pathway, could take over the role of R2D2 in these basal insect species. This assumption, which was already suggested by Christiaens et al. (2015) and Taning et al. (2016a) is also supported by reports of Loquacious being functional as cofactor to Dicer-2 in the siRNA pathway in D. melanogaster (Czech et al., 2008; Marques et al., 2010; Okamura et al., 2008). In Crustacea, another protein has been identified as a cofactor for Dicer-2, namely an orthologue of the transactivating response (TAR) RNA binding protein (TRBP) (Lai and Aboobaker, 2017).

It is difficult to say whether these differences regarding R2D2 could be linked to RNAi efficiency in these species. Swevers et al. (2013b) have suggested that this could be the case, based on the observations that the RNAi sensitive Coleoptera possess two R2D2 copies (Swevers et al., 2013b), while less sensitive Lepidoptera have either no R2D2, or the gene is only expressed at very low levels as is the case in B. mori (Swevers et al., 2011). However, Diaphorini citri is sensitive to RNAi despite the absence of an R2D2 gene in the genome (Taning et al., 2016a). However, these authors did indicate that, despite quantitative differences in terms of RNAi-related genes, there was no qualitative difference in terms of functional groups that were represented in these nematode species. Furthermore, they could also not identify a link between RNAi effector repertoire and RNAi functionality in parasitic nematodes.

The present document has been produced and adopted by the bodies identified above as authors. This task has been carried out exclusively by the author(s) in the context of a contract between the European Food Safety Authority and the authors, awarded following a tender procedure. The present document is published complying with the transparency principle to which the Authority is subject. It may not be considered as an output adopted by the Authority. The European Food Safety Authority reserves its rights, view and position as regards the issues addressed and the conclusions reached in the present document, without prejudice to the rights of the authors. 


\subsubsection{Persistence of dsRNA in the body}

One of the major factors contributing to the variability in RNAi efficiency in invertebrates, especially insects, is the stability and persistence of dsRNA in the invertebrate body. Many studies have demonstrated that dsRNA can be rapidly degraded by nucleolytic activity in the saliva, the intestinal fluid or the haemolymph of a wide range of insect species (Allen and Walker, 2012; Arimatsu et al., 2007; Christiaens et al., 2014; Garbutt et al., 2013; Liu et al., 2012; Luo et al., 2013; Wynant et al., 2014a; Wynant et al., 2014b). In the pea aphid for example, researchers showed that the secreted saliva of an aphid into its artificial diet, was able to cause degradation of dsRNA in the diet. Additionally, the haemolymph of these plant suckers was also able to degrade dsRNA (Christiaens et al., 2014). The tarnished plant bug $L$. lineolaris and the Southern green stinkbug, saliva was also found to rapidly degrade dsRNA molecules (Allen and Walker, 2012; Lomate and Bonning, 2016). Even more evidence exists for degradation in the gut lumen. Studies in caterpillars of $M$. sexta (Garbutt et al., 2013), B. mori (Arimatsu et al., 2007; Liu et al., 2012), in African sweet potato weevils C. puncticollis and C. brunneus (Prentice et al., 2017), in the locusts S. gregaria and L. migratoria (Luo et al., 2013; Wynant et al., 2014b) and even in the Colorado potato beetle, which is considered very sensitive for oral RNAi, degradation of the dsRNA in the gut has been shown (Prentice et al., 2017; Spit et al., 2017). The fact that dsRNA degradation can be observed in the intestinal fluid of CPB, which is very sensitive to oral RNAi, shows that degrading nucleolytic activity in the gut does not necessarily mean that a species is insensitive to RNAi, but merely that RNAi efficiency can be compromised. Since these experiments were all carried out in vitro, often with extracted intestinal fluid from dissected midguts, it is difficult to say at what speed the dsRNA actually degrades in the gut in an in vivo situation. It is also difficult to compare stabilities between different studies, since gut juice is often collected with different methods, in different dilutions and the incubated dose of dsRNA differs as well. But it is clear that the persistence in some insects is higher than in others, and in some cases, this can be associated with a difference in RNAi efficiency. A recent publication by Prentice et al. (2017) provided data comparing the RNAi responses in different beetle species and comparing them with dsRNA persistence in intestinal fluid. They found a clear correlation between RNAi efficiency and the speed of degradation of the dsRNA. Notably the difference between the Colorado potato beetle, which is very sensitive to oral RNAi, and the sweet potato weevils $C$. puncticollis and $C$. brunneus, which are less sensitive, was striking. While dsRNA in sweet potato weevil extracts remained stable for 30 minutes to one hour, intact fragments could still be detected after 16 hours in CPB. Additionally, the difference in dsRNA stability in both weevil species corresponded to a difference in RNAi efficiency upon feeding with dsRNA (Prentice et al., 2017). Further evidence was also delivered when Spit et al. (2017) were able to improve RNAi sensitivity after silencing certain nucleases present in the gut.

A number of studies have also looked into the nucleases that could be implicated in this phenomenon. In the silkworm $B$. mori, a DNA/RNA non-specific nuclease was discovered that was highly expressed in the gut and was able to degrade dsRNA rapidly (Arimatsu et al., 2007; Liu et al., 2012). In fact, it was later shown that expression of this dsRNase is upregulated upon injection of dsRNA in B. mori (Liu et al., 2013). The authors hypothesized that this might be related to an antiviral mechanism. In M. sexta, Garbutt et al. (2013) concluded, based on their characterization studies, that the nucleolytic breakdown of dsRNA in the gut is caused by one or more metallo-enzymes, since addition of EDTA was able to stop degradation of the dsRNA. In S. gregaria and L. decemlineata, several nucleases were identified in the transcriptome that were able to degrade dsRNA (Spit et al., 2017; Wynant et al., 2014b). Interestingly, when these nuclease-encoding genes were silenced in both species, RNAi efficiency increased in CPB, but the S. gregaria locusts remained insensitive to RNAi. Analysis of the gut juice of these locusts showed that silencing the identified nucleases increased dsRNA persistence, but degradation could still be observed in the 50x diluted gut juice after 10 minutes incubation. This suggests that other nucleases might also be present in the locust which were not identified in these studies (Spit et al., 2017; Wynant et al., 2014b). In the Southern green stinkbug, N. viridula, researchers investigated the transcriptome, searching for potential dsRNA-degrading nucleases

The present document has been produced and adopted by the bodies identified above as authors. This task has been carried out exclusively by the author(s) in the context of a contract between the European Food Safety Authority and the authors, awarded following a tender procedure. The present document is published complying with the transparency principle to which the Authority is subject. It may not be considered as an output adopted by the Authority. The European Food Safety Authority reserves its rights, view and position as regards the issues addressed and the conclusions reached in the present document, without prejudice to the rights of the authors. 
(Lomate and Bonning, 2016). They found very strong dsRNA degradation activity in the saliva, while much lower activity was observed in the gut and salivary glands. An explanation for the difference between activity in the saliva and the salivary glands could be that the enzymes are released as zymogens, and are only activated once they are secreted in the saliva. Given the (extraoral) digestion behaviour of these plant sucking pest, by injecting saliva into the plant, the fact that the nuclease activity is higher in saliva than in the gut might not be surprising (Lomate and Bonning, 2016).

While a large body of evidence exists on dsRNA instability in insects and nucleolytic degradation in the digestive system, we were not able to find any studies investigating sRNA stability in nematodes, molluscs and annelids.

\subsubsection{SRNA molecule}

The characteristics of the SRNA molecule to which the invertebrate is exposed could also have a major impact on RNAi efficiency. For example, it is known that, in the nematode $C$. elegans and in some insects, cellular uptake efficiency is dependent on the dsRNA length. Length dependency in nematodes was already discussed in Section 5.1 in relation to the selectivity for dsRNA length exhibited by Sid-2. To briefly summarize, studies have shown that dsRNA longer than 25bp requires much lower concentrations than siRNA to have a similar RNAi effect and that this is due to Sid-2 being selective for length in uptake from the environment (Feinberg and Hunter, 2003; McEwan et al., 2012). However, nine studies were found reporting successful RNAi using siRNA soaking in nematodes, for example in M. incognita (Arguel et al., 2012; Dalzell et al., 2010a; Dalzell et al., 2010b), B. xylophilus (Ma et al., 2011) M. graminicolis (Haegeman et al., 2013), G. pallida (Dalzell et al., 2010a) and C. elegans (Sivamaruthi and Balamurugan, 2014).

In insects, similar observations have been reported. Saleh et al. (2006) found that long dsRNA were efficiently taken up in Drosophila S2 cells, while siRNAs required a transfection agent to be internalized. Bolognesi et al. (2012) compared cellular uptake of a Cy3-labeled long dsSnf7 fragment (240 bp) with that of a Cy3-labeled siRNA targeting Snf7 in D. virgifera midgut cells, and found that only the $240 \mathrm{bp}$ fragment was taken up into the cells. These findings were also supported by biological activity data, since feeding of the long fragment led to mortality while feeding of the siRNA had no effect. In experiments with the Southern corn rootworm (SCR), Bolognesi et al. (2012) tested dsRNAs of different lengths and observed mortality from dsRNA ingestion increase from $16 \%$ for a $50 \mathrm{bp}$ fragment to $68 \%$ mortality feeding a $60 \mathrm{bp}$ fragment and eventually to $95 \%$ mortality using a $70 \mathrm{bp}$ fragment. These results suggest that for SCR, the minimum length for efficient RNAi is around $60 \mathrm{bp}$. Important to note here is that the authors used only a single $27 \mathrm{nt}$ sequence that was specific for the target insect in each of their dsRNAs, so these effects are not merely the result of having more specific siRNAs in the cells (Bolognesi et al., 2012). Miller et al. (2012) reported similar observations in $T$. castaneum, where 65 bp dsRNA fragments were successful in silencing a GFP construct, while 31 bp dsRNAs were not.

It is not yet clear whether this is a characteristic shared by all insects, let alone other invertebrates. For example, several publications have reported the use of 20-23 bp siRNA in RNAi experiments in a range of insect species, including the honeybee $A$. mellifera (Chen et al., 2014c), the diamondback moth P. xylostella (Gong et al., 2013), the cotton bollworm H. armigera (Zhang et al., 2015c), the pea aphid $A$. pisum (Mutti et al., 2006), the whitefly B. tabaci (Upadhyay et al., 2011), the potato/tomato psyllid Bactericerca cockerelli (Wuriyanghan et al., 2011), termites (Zhou et al., 2006) and several others. Therefore, it appears to be probable that there are differences between different taxonomical clades concerning the influence of dsRNA length on RNAi efficiency. It is also important to note that the siRNA in some of these experiments was produced by in vitro dicing of dsRNA, meaning that a very small residual amount of long dsRNA might still have been present. However, most studies used chemically synthesized siRNA. In Table 13, we give an overview of invertebrate orders for which successful RNAi, using siRNA, has been reported. Remarkably, many of the insect species where

The present document has been produced and adopted by the bodies identified above as authors. This task has been carried out exclusively by the author(s) in the context of a contract between the European Food Safety Authority and the authors, awarded following a tender procedure. The present document is published complying with the transparency principle to which the Authority is subject. It may not be considered as an output adopted by the Authority. The European Food Safety Authority reserves its rights, view and position as regards the issues addressed and the conclusions reached in the present document, without prejudice to the rights of the authors. 
siRNA has proven to successfully elicit RNAi were Hemiptera, possibly suggesting that siRNA works more efficiently in Hemiptera than in other orders.

Table 13: Overview of type of sRNA used to successfully elicit gene silencing by oral feeding

\begin{tabular}{lllll}
\hline Class & Order & siRNA & dsRNA & miRNA (mimic) \\
\hline Hexapoda & Diptera & $\left(^{*}\right)$ & $\left(^{*}\right)$ & + \\
& Lepidoptera & + & + & + \\
& Coleoptera & - & + & N/A \\
& Hymenoptera & + & + & + \\
& Hemiptera & + & + & + \\
& Orthoptera & + & + & N/A \\
Crustacea & Blattodea & + & + & N/A \\
Chelicerata & & - & + & N/A \\
Adenophorea & & N/A & + & N/A \\
Secernentea & & N/A & + & N/A \\
\hline
\end{tabular}

* Oral RNAi experiments in Diptera required polymer- or liposome-based formulations to elicit successful knockdown, both for siRNA and longer dsRNA

N/A: No studies were found exposing the invertebrate to this SRNA orally

Interesting to note in this context is that in GM plants expressing dsRNA, the plant RNAi machinery appears to process most expressed pest-specific dsRNA to siRNAs. Given the successes that have been reported using these insect-specific dsRNA expressing plants to induce gene silencing in the target insect, for example in Hemiptera (Abdellatef et al., 2015; Bhatia et al., 2012; Coleman et al., 2011; Coleman et al., 2014; Coleman et al., 2015; Khan et al., 2015; Khan et al., 2013; Raza et al., 2016; Zha et al., 2011), Lepidoptera (Apone et al., 2014; Kumar et al., 2012; Liu et al., 2015; Tian et al., 2015a; Zhu et al., 2012) and Coleoptera (Baum et al., 2007; Li et al., 2015a), it raises the question of whether siRNA is causing the gene silencing or residual dsRNA in the plant, not yet processed by the plant's RNAi machinery. In several studies, target RNA characterization of the transgenic plants showed that most dsRNA is processed by the plant into siRNAs. Li et al. (2015a) found a majority of $21 \mathrm{bp}-24 \mathrm{bp}$ fragments but also a clear band for the intact $275 \mathrm{nt}$ dsRNA fragment that was used in this study. Similar observations of plant processing of the dsRNA were made in other studies (Pitino et al., 2011; Thakur et al., 2014; Wuriyanghan and Falk, 2013; Zha et al., 2011). Interestingly, an in planta study using tobacco plants to express dsRNA specifically targeting a M. sexta cytochrome P450 (CYP) gene, found that gene silencing in the herbivorous insect was more efficient when the plant Dicer enzyme was silenced, providing further evidence of the importance of dsRNA length for efficient RNAi in insects (Kumar et al., 2012). Similarly, in another study using Dicer-mutant Arabidopsis lines, higher levels of dsRNA accumulation and less processing into siRNA in the mutant plants compared to wild type Arabidopsis plants, was accompanied by a higher RNAi efficiency in the cotton bollworm feeding on these plants (Mao et al., 2007).

The exact mechanism for this length dependency is unclear. In nematodes, Sid-2 has been shown to import dsRNA of greater length more efficiently than siRNAs. Possibly a similar mechanism is at work in arthropods through the sid-1-like uptake route. Uptake of siRNA through the endocytosis route could then explain why siRNAs can also still incite RNAi, but less efficiently than long dsRNA. But this is speculation at this moment. Further research at the cell level should be able to provide more information on the uptake capacity in invertebrates in the future. Recently, the production of dsRNA in the chloroplasts of the plant was reported as a way to increase RNAi efficiency against target

The present document has been produced and adopted by the bodies identified above as authors. This task has been carried out exclusively by the author(s) in the context of a contract between the European Food Safety Authority and the authors, awarded following a tender procedure. The present document is published complying with the transparency principle to which the Authority is subject. It may not be considered as an output adopted by the Authority. The European Food Safety Authority reserves its rights, view and position as regards the issues addressed and the conclusions reached in the present document, without prejudice to the rights of the authors. 
herbivores. The main advantage was that dsRNA accumulated in these chloroplasts and was not processed by the plant's own RNAi system (Jin et al., 2015; Zhang et al., 2015b). Another element to take into account here is that siRNAs are modified with 2 'O-methylation when processed by the plant RNAi machinery, to protect them from nuclease activity. Whether this has any impact on the stability of these siRNAs in the invertebrate body when confronted with a nucleolytic environment is uncertain (Chan and Snow, 2017). If it does, this might compensate for the potentially less efficient uptake of these siRNAs by invertebrates.

Another characteristic of the dsRNA molecule that could influence RNAi efficiency is the location of the site/region in the gene that the dsRNA is homologous to. Several research studies have looked at the possibility of designing dsRNAs in the $3^{\prime}$ untranslated region (UTR) or $5^{\prime}$ UTR. The advantage of choosing these regions are that they are much less conserved than the part of the coding sequence (CDS) that is translated into protein, leading to a potentially much higher specificity of the pesticidal molecule. However, variable efficacies have been reported. For example, in a study targeting the inhibitor of apoptosis gene in $A$. aegypti, researchers compared the efficacies of different dsRNAs targeting different regions of the target gene and found that the $3^{\prime}$ end targeting dsRNA had a greater effect on mosquito mortality than the dsRNA targeting the $5^{\prime}$ end (Pridgeon et al., 2008). In contrast, Loy et al. (2012) found that the most effective dsRNA targeting the infectious myonecrosis virus in shrimp was the one targeting the $5^{\prime}$ end. Finally, a study in the pea aphid $A$. pisum revealed no differences in efficiency between dsRNA targeting the $3^{\prime}$ end and the $5^{\prime}$ end of the hunchback gene (Mao and Zeng, 2012). These data demonstrate that target gene region can be an important factor affecting RNAi efficiency, and these will have to be determined empirically for every case.

\subsubsection{Cellular uptake of dsRNA}

dsRNA cellular uptake efficiency varies between different species. For example, in nematodes, research has shown that $C$. elegans possesses an expanded gene repertoire encoding proteins which have different functions in cellular uptake and systemic spread, including several sid-like genes. However, many parasitic nematodes appear to have a more limited set of effectors in dsRNA uptake and systemic spread, possible having an impact on RNAi efficiency (Dalzell et al., 2011). In insects, two different cellular uptake pathways have been identified (Cappelle et al., 2016; Saleh et al., 2006; Tomoyasu et al., 2008; Ulvila et al., 2006). One is based on clathrin-mediated endocytosis, while another involves a SID-1-like protein. Whether both pathways are completely independent is still unsure, but research in several species that have both pathways functional has shown that inhibition of one of these negatively impacts RNAi efficiency. Diptera, comprising flies and mosquitoes, do not possess a sid-1-like homologue and appear to rely solely on their endocytosis mechanism for cellular uptake of dsRNA. Research in S2 cells has demonstrated that expression of sid-1 could significantly enhance the uptake of dsRNA. Additionally, feeding RNAi studies in D. melanogaster and D. suzukii suggest that cellular uptake is a major reason why these fruit flies are refractory to oral RNAi (Taning et al., 2016b; Whyard et al., 2009). Recently, researchers found that in two lepidopteran cell lines, $S$. frugiperda Sf9 and $H$. virescens HvE6, RNAi was taken up from the medium, but never processed into siRNA, unlike in coleopteran cells. These results suggest that intracellular release of dsRNA is an additional problem in Lepidoptera possibly explaining their insensitivity for RNAi (Shukla et al., 2016).

There is a more detailed discussion on cellular uptake mechanisms and systemic spread of sRNAs, in Section 5.1 .

\subsubsection{Amplification of the RNAi signal}

One of the important factors influencing the efficiency of RNAi in the nematode $C$. elegans is the presence of an amplification system for the RNAi silencing signal, like that in plants. In $C$. elegans, an $\mathrm{RdRP}$ is at the center of this process, eventually leading to the production of secondary siRNAs. The mechanism itself has been discussed in depth in Section 5.1. Thus far, no homologue of this RdRP has been found in any insect or crustacean genome. There is however evidence for the presence of

The present document has been produced and adopted by the bodies identified above as authors. This task has been carried out exclusively by the author(s) in the context of a contract between the European Food Safety Authority and the authors, awarded following a tender procedure. The present document is published complying with the transparency principle to which the Authority is subject. It may not be considered as an output adopted by the Authority. The European Food Safety Authority reserves its rights, view and position as regards the issues addressed and the conclusions reached in the present document, without prejudice to the rights of the authors. 
this RdRP in certain tick species (Kurscheid et al., 2009). Whether or not this means that these ticks can amplify silencing RNAs, as in C. elegans, is not yet clear. Additionally, the absence of this RdRP does not necessarily mean that some species of insects or Crustacea do not possess a different mechanism to amplify the silencing signal. An alternative amplification mechanism could explain why in some species long lasting gene silencing or even a strong parental RNAi response can be observed. In fact, recent research in D. melanogaster has identified a siRNA amplification system, where secondary siRNAs are synthesized from viral dsRNA upon infection, via viral cDNAs. However, the study reported that this amplification mechanism was only activated in the presence of viruses and might therefore not be involved in an amplification of siRNA derived from delivery of exogenous dsRNA (Tassetto et al., 2017).

\subsubsection{Viral infections}

In invertebrates and plants, RNAi is a major component of the innate antiviral immunity response (Ding, 2010; Keene et al., 2004; Lu et al., 2005; Robalino et al., 2005; Schott et al., 2005; Sidahmed et al., 2014; van Rij et al., 2006; Wilkins et al., 2005). Several studies have shown that injection of non-specific dsRNA is able to activate this RNAi response, for example by upregulation of RNAi machinery core genes Dicer-2 and Ago-2 (Garbutt and Reynolds, 2012; Liu et al., 2013; Lozano et al., 2012). In B. mori, injection of dsRNA also led to the upregulation of a dsRNase capable of degrading dsRNA in the silkworm. A similar effect was seen when B. mori larvae were infected with B. mori cytoplasmi polyhedrosis virus (BmCPV), which is characterized by a genome of linear dsRNA elements (Wu et al., 2009). Given the close relationship between viruses and RNAi, it would not be surprising that viruses which have co-evolved with this line of defense, have developed mechanisms to inhibit the RNAi machinery. As hypothesized by Swevers et al. (2013a), such mechanisms could entail the production of viral suppressors of RNAi (VSRs) and saturation of the RNAi machinery due to accumulation of large quantities of viRNAs.

The first discovery of a VSR in invertebrate viruses was in Flock House Virus, which encodes the socalled B2 protein, capable of interfering with the RNAi machinery in different ways, including binding to dsRNA and siRNA, making it unavailable to be processed by the RNAi machinery, and directly interacting with Dicer-2 (Aliyari et al., 2008; Chao et al., 2005; Li et al., 2002; Singh et al., 2009). Another example was found with Drosophila $C$ virus, which encodes an $1 A$ protein capable of binding to dsRNA and, to a lesser extent, siRNA (van Rij et al., 2006). In Cricket Paralysis virus (CrPV) infections, the A1 protein was found not to bind to dsRNA/siRNA but was able to directly interact with Ago-2 and interfere with the function of RISC (Nayak et al., 2010). Interestingly, a study on bumblebees showed that infection with CrPV had no apparent effect on RNAi efficiency, while Israeli Acute Paralysis Virus infection actually enhanced RNAi efficiency (Cappelle et al., 2017). Also in human virus diseases, such as Dengue and West Nile virus, VSRs have been reported (Kakumani et al., 2013; Schnettler et al., 2012). Finally, VSRs in the form of nucleases have also been found to be expressed by invertebrate viruses. For example in Heliothis virescens ascovirus (HvAV) infections, an RNase III enzyme was found to be expressed capable of degrading dsRNA and siRNA and negatively affecting RNAi efficiency in the host (Hussain et al., 2010).

In vertebrates, a different mechanism based on the expression of a non-coding RNA by adenoviruses has been discovered allowing these viruses to suppress the RNAi response (by inhibiting siRNA and miRNA production) in adenovirus-infected vertebrate cells. This happens by interfering with nuclear export of short hairpin (shRNA) or pre-microRNA precursors, by competition for the Exportin 5 nuclear export factor or by interaction with Dicer function by direct binding to Dicer (Lu and Cullen, 2004). In contrast, production of so-called defective interfering RNAs (diRNAs) by a plant tombovirus actually enhanced RNAi efficiency due to hindering of the activity of VSRs produced by the virus (Havelda et al., 2005; Pathak and Nagy, 2009). Recently, suppression of RNAi in tick and insect cells by viRNAs produced by flaviviruses was also discovered, although the exact mechanism is not known yet (Schnettler et al., 2012; Schnettler et al., 2014).

The present document has been produced and adopted by the bodies identified above as authors. This task has been carried out exclusively by the author(s) in the context of a contract between the European Food Safety Authority and the authors, awarded following a tender procedure. The present document is published complying with the transparency principle to which the Authority is subject. It may not be considered as an output adopted by the Authority. The European Food Safety Authority reserves its rights, view and position as regards the issues addressed and the conclusions reached in the present document, without prejudice to the rights of the authors. 
Whether viral suppression of the RNAi machinery is a major problem in field populations of invertebrates is unclear. It could be one of the factors explaining variation sometimes observed between different strains or even different laboratory cultures of the same strain, such as the pea aphid which is a vector for several plant viruses (Christiaens and Smagghe, 2014). Interestingly, Swevers et al. (2013a) report that the vast majority of insect viruses are found in Lepidoptera, which are less sensitive to RNAi, while the RNAi sensitive Coleoptera are characterized by very low occurrences of viral infections. However, whether this observation indicates any causality is still to be determined. A study on lepidopteran cell lines indicated no apparent effect on RNAi efficiency upon persistent infection by viruses known to encode VSRs (Swevers et al., 2016).

\subsubsection{Invertebrate life stage}

A few studies of the influence of developmental stage of invertebrates on RNAi efficiency were found. These include Terenius et al. (2011) who reported that in some experiments on lepidopteran insects, adults were more sensitive to RNAi when injected than juvenile stages. However, these observations should be confirmed by experiments using identical experimental conditions and setup. In contrast, some studies suggested that RNAi worked more efficiently in earlier juvenile stages than in later stages. For example, in the kissing bug $R$. prolixus no knockdown was observed when treating $4^{\text {th }}$ instars with $2 \times 80 \mu \mathrm{g}$ nitropin2 dsRNA orally while $42 \%$ silencing was achieved in second instar individuals which had ingested approximately $13 \mu \mathrm{g}$ dsNRA on average (Araujo et al., 2006). Additionally, Griebler et al. (2008) also reported higher RNAi efficiency in larvae compared to adults of the fall armyworm S. frugiperda. Obviously, juvenile stages feed more than adult stages, which means that in most experiments with an ad libitum dsRNA supply, they will be exposed to a much higher dose of dsRNA. However, taking into account the dose of dsRNA/gram body weight, it seems that younger juvenile instars were more susceptible than older juvenile instars in some of these studies. Nevertheless, very little actual data is available and more research into this phenomenon is necessary before a more complete picture can be drawn.

\subsubsection{Protein stability or protein half life}

Protein half-life varies enormously. For example in mammals, the half-life of certain collagen proteins have been reported to be longer than 100 years, while ornithine carboxylase has a reported half-life of 22 minutes (Iwami et al., 1990; Verzijl et al., 2000). Moreover, protein half-lives for the same protein can also vary between different species (Kuhar and Joyce, 2001). While this factor does not influence silencing at the transcript level per se, the stability or half-life of the protein encoded by the gene targeted for knockdown determines the degree in which phenotypical effects could be observed and is therefore something that has to be taken account when choosing target genes in a certain invertebrate species. Unfortunately, we found no reports on half-lives of invertebrate proteins associated with targets for RNAi and information on half-lives for most proteins are still unknown.

\subsubsection{Conclusions}

In this section, we provided an overview of the literature related to RNAi efficiency in invertebrates, which shows that it is very variable, not only between species, but also sometimes between strains, (laboratory) cultures, life stage or due to experimental aspects such as the sRNA molecule that is used. We have considered and discussed a number of factors and mechanisms that are known to affect the ability and hence the efficiency to induce RNAi-based gene silencing. While some of these factors, such as dsRNA stability in the insect body and cellular uptake, have attracted a lot of attention from researchers, a number of other factors such as the influence of the life stage or the impact of viral infections have not been studied in depth.

The present document has been produced and adopted by the bodies identified above as authors. This task has been carried out exclusively by the author(s) in the context of a contract between the European Food Safety Authority and the authors, awarded following a tender procedure. The present document is published complying with the transparency principle to which the Authority is subject. It may not be considered as an output adopted by the Authority. The European Food Safety Authority reserves its rights, view and position as regards the issues addressed and the conclusions reached in the present document, without prejudice to the rights of the authors. 


\subsubsection{References}

Abd El Halim HM, Alshukri BMH, Ahmad MS, et al., 2016. RNAi-mediated knockdown of the voltage gated sodium ion channel TcNav causes mortality in Tribolium castaneum. Scientific Reports 6, 29301-.

Abdellatef E, Will T, Koch A, Imani J, Vilcinskas A, Kogel K-H, 2015. Silencing the expression of the salivary sheath protein causes transgenerational feeding suppression in the aphid Sitobion avenae. Plant Biotechnology Journal 13, 849-57.

Aboobaker $A A$, Blaxter $M L, 2003$. Use of RNA interference to investigate gene function in the human filarial nematode parasite Brugia malayi. Molecular and Biochemical Parasitology 129, 41-51.

Aboobaker AA, Blaxter ML, 2004. Functional genomics for parasitic nematodes and platyhelminths. Trends in Parasitology 20, 178-84.

Adams MD, Celniker SE, Holt RA, et al., 2000. The genome sequence of Drosophila melanogaster. Science 287, 2185-95.

Agrawal A, Rajamani V, Reddy VS, Mukherjee SK, Bhatnagar RK, 2015. Transgenic plants overexpressing insect-specific microRNA acquire insecticidal activity against Helicoverpa armigera: an alternative to Bt-toxin technology. Transgenic Research 24, 791-801.

Al-Amin M, Kawasaki I, Gong J, Shim Y-H, 2016. Caffeine Induces the Stress Response and UpRegulates Heat Shock Proteins in Caenorhabditis elegans. Molecules and Cells 39, 163-8.

Aliyari R, Wu Q, Li H-W, et al., 2008. Mechanism of Induction and Suppression of Antiviral Immunity Directed by Virus-Derived Small RNAs in Drosophila. Cell Host \& Microbe 4, 387-97.

Allen ML, Walker WB, Iii, 2012. Saliva of Lygus lineolaris digests double stranded ribonucleic acids. Journal of Insect Physiology 58, 391-6.

Apone F, Ruggiero A, Tortora A, et al., 2014. Targeting the diuretic hormone receptor to control the cotton leafworm, Spodoptera littoralis. Journal of Insect Science 14.

Araujo RN, Santos A, Pinto FS, Gontijo NF, Lehane MJ, Pereira MH, 2006. RNA interference of the salivary gland nitrophorin 2 in the triatomine bug Rhodnius prolixus (Hemiptera : Reduviidae) by dsRNA ingestion or injection. Insect Biochemistry and Molecular Biology 36, 683-93.

Arguel M-J, Jaouannet M, Magliano M, Abad P, Rosso M-N, 2012. siRNAs Trigger Efficient Silencing of a Parasitism Gene in Plant Parasitic Root-Knot Nematodes. Genes 3, 391-408.

Arimatsu Y, Furuno T, Sugimura Y, et al., 2007. Purification and properties of double-stranded RNAdegrading nuclease, dsRNase, from the digestive juice of the silkworm, Bombyx mori. Journal of Insect Biotechnology and Sericology 76, 57-62.

Attasart $P$, Namramoon $O$, Kongphom $U$, Chimwai $C$, Panyim S, 2013. Ingestion of bacteria expressing dsRNA triggers specific RNA silencing in shrimp. Virus Research 171, 252-6.

Ayra-Pardo C, Raymond B, Gulzar A, et al., 2015. Novel genetic factors involved in resistance to Bacillus thuringiensis in Plutella xylostella. Insect Molecular Biology 24, 589-600.

Bakhetia M, Urwin PE, Atkinson $\mathrm{HJ}$, 2007. qPCR analysis and RNAi define pharyngeal gland cellexpressed genes of Heterodera glycines required for initial interactions with the host. Molecular Plant-Microbe Interactions 20, 306-12.

Bansal R, Michel AP, 2013. Core RNAi Machinery and Sid1, a Component for Systemic RNAi, in the Hemipteran Insect, Aphis glycines. International Journal of Molecular Sciences 14, 3786-801.

The present document has been produced and adopted by the bodies identified above as authors. This task has been carried out exclusively by the author(s) in the context of a contract between the European Food Safety Authority and the authors, awarded following a tender procedure. The present document is published complying with the transparency principle to which the Authority is subject. It may not be considered as an output adopted by the Authority. The European Food Safety Authority reserves its rights, view and position as regards the issues addressed and the conclusions reached in the present document, without prejudice to the rights of the authors. 
Baqri R, Charan R, Schimmelpfeng K, Chavan S, Ray K, 2006. Kinesin-2 differentially regulates the anterograde axonal transports of acetylcholinesterase and choline acetyltransferase in Drosophila. Journal of Neurobiology 66, 378-92.

Barreto FS, Schoville SD, Burton RS, 2015. Reverse genetics in the tide pool: knock-down of target gene expression via RNA interference in the copepod Tigriopus californicus. Molecular Ecology Resources 15, 868-79.

Baum JA, Bogaert T, Clinton W, et al., 2007. Control of coleopteran insect pests through RNA interference. Nature Biotechnology 25, 1322-6.

Baum JA, Roberts JK, 2014. Progress Towards RNAi-Mediated Insect Pest Management. In: Dhadialla TS, Gill SS, eds. Insect Midgut and Insecticidal Proteins. 249-95. (Advances in Insect Physiology; vol. 47.)

Bettencourt R, Terenius O, Faye I, 2002. Hemolin gene silencing by ds-RNA injected into Cecropia pupae is lethal to next generation embryos. Insect Molecular Biology 11, 267-71.

Bhatia V, Bhattacharya R, Uniyal PL, Singh R, Niranjan RS, 2012. Host Generated siRNAs Attenuate Expression of Serine Protease Gene in Myzus persicae. PLoS ONE 7.

Bolognesi R, Ramaseshadri P, Anderson J, et al., 2012. Characterizing the Mechanism of Action of Double-Stranded RNA Activity against Western Corn Rootworm (Diabrotica virgifera virgifera LeConte). PLOS ONE 7.

Bona ACD, Chitolina RF, Fermino ML, et al., 2016. Larval application of sodium channel homologous dsRNA restores pyrethroid insecticide susceptibility in a resistant adult mosquito population. Parasites \& Vectors 9, 397-.

Cancino-Rodezno A, Alexander C, Villasenor R, et al., 2010. The mitogen-activated protein kinase p38 is involved in insect defense against Cry toxins from Bacillus thuringiensis. Insect Biochemistry and Molecular Biology 40, 58-63.

Cancino-Rodezno A, Lozano L, Oppert C, et al., 2012. Comparative Proteomic Analysis of Aedes aegypti Larval Midgut after Intoxication with Cry11Aa Toxin from Bacillus thuringiensis. PLoS ONE 7.

Cappelle K, De Oliveira CFR, Van Eynde B, Christiaens O, Smagghe G, 2016. The involvement of clathrin-mediated endocytosis and two Sid-1-like transmembrane proteins in doublestranded RNA uptake in the Colorado potato beetle midgut. Insect Molecular Biology 25, 315-23.

Cappelle K, Smagghe G, Dhaenens M, Meeus I, 2016. Israeli acute paralysis virus infection leads to an enhanced rna interference response and not its suppression in the bumblebee Bombus terrestris. Viruses 8, 1-17

Chan SY, Snow JW, 2017. Uptake and impact of natural diet-derived small RNA in invertebrates: Implications for ecology and agriculture. RNA biology 14, 402-14.

Chao JA, Lee JH, Chapados BR, Debler EW, Schneemann A, Williamson JR, 2005. Dual modes of RNAsilencing suppression by flock house virus protein B2. Nature Structural \& Molecular Biology 12, 952-7.

Chapman EJ, Carrington JC, 2007. Specialization and evolution of endogenous small RNA pathways. Nature Reviews Genetics 8, 884-96.

Chen A, Zheng W, Zheng W, Zhang $H, 2015$. The effects of RNA interference targeting Bactrocera dorsalis ds-Bdrpl19 on the gene expression of rpl19 in non-target insects. Ecotoxicology 24, 595603.

The present document has been produced and adopted by the bodies identified above as authors. This task has been carried out exclusively by the author(s) in the context of a contract between the European Food Safety Authority and the authors, awarded following a tender procedure. The present document is published complying with the transparency principle to which the Authority is subject. It may not be considered as an output adopted by the Authority. The European Food Safety Authority reserves its rights, view and position as regards the issues addressed and the conclusions reached in the present document, without prejudice to the rights of the authors. 
Chen $H$, Yang $X$, Tang T, et al., 2014a. The involvement of cysteine-rich intestinal protein in early development and innate immunity of Asiatic hard clam, Meretrix meretrix. Fish \& Shellfish Immunology 40, 435-40.

Chen J, Zhang D, Yao Q, et al., 2010. Feeding-based RNA interference of a trehalose phosphate synthase gene in the brown planthopper, Nilaparvata lugens. Insect Molecular Biology 19, 777-86.

Chen R, Ren X, Han Z, et al., 2014b. A cadherin-like protein from the beet armyworm Spodoptera exigua (Lepidoptera: Noctuidae) is a putative Cry1Ac receptor. Archives of Insect Biochemistry and Physiology 86, 58-71.

Chen X, Yang Y, Yang J, Zhang Z, Zhu X, 2012. RNAi-Mediated Silencing of Paramyosin Expression in Trichinella spiralis Results in Impaired Viability of the Parasite. PLOS ONE 7.

Chen $\mathrm{Y}-\mathrm{H}$, Jia X-T, Zhao $\mathrm{L}$, et al., 2011. Identification and functional characterization of Dicer2 and five single VWC domain proteins of Litopenaeus vannamei. Developmental and Comparative Immunology 35, 661-71.

Chen YP, Pettis JS, Corona M, et al., 2014c. Israeli Acute Paralysis Virus: Epidemiology, Pathogenesis and Implications for Honey Bee Health. PLoS pathogens 10.

Christiaens O, Delbare D, Van Neste C, et al., 2015. Differential transcriptome analysis of the common shrimp Crangon crangon: Special focus on the nuclear receptors and RNAi-related genes. General and Comparative Endocrinology 212, 163-77.

Christiaens O, Prentice K, Pertry I, et al., 2016. RNA interference: a promising biopesticide strategy against the African Sweetpotato Weevil Cylas brunneus. Scientific Reports 6, 38836.

Christiaens O, Smagghe G, 2014. The challenge of RNAi-mediated control of hemipterans. Current Opinion in Insect Science 6, 15-21.

Christiaens O, Swevers L, Smagghe G, 2014. DsRNA degradation in the pea aphid (Acyrthosiphon pisum) associated with lack of response in RNAi feeding and injection assay. Peptides 53, 307-14.

Coleman AD, Hogenhout SA, Pitino M, Maffei ME, Ridout CJ, 2011. Silencing of Aphid Genes by dsRNA Feeding from Plants. Figshare.

Coleman AD, Pitino M, Hogenhout SA, 2014. Silencing of Aphid Genes by Feeding on Stable Transgenic Arabidopsis thaliana. In: Birch P, Jones JT, Bos JIB, eds. Plant-Pathogen Interactions: Methods and Protocols, 2nd Edition. 125-36. (Methods in Molecular Biology; vol. 1127.)

Coleman AD, Wouters RHM, Mugford ST, Hogenhout SA, 2015. Persistence and transgenerational effect of plant-mediated RNAi in aphids. Journal of Experimental Botany 66, 541-8.

Conzelmann M, Williams EA, Tunaru S, et al., 2013. Conserved MIP receptor-ligand pair regulates Platynereis larval settlement. Proceedings of the National Academy of Sciences 110, 8224-9.

Cruz J, Mane-Padros D, Belles X, Martin D, 2006. Functions of the ecdysone receptor isoform-A in the hemimetabolous insect Blattella germanica revealed by systemic RNAi in vivo. Developmental Biology 297, 158-71.

Czech $B$, Malone $C D$, Zhou $R$, et al., 2008. An endogenous small interfering RNA pathway in Drosophila. Nature 453, 798-U7.

Dalzell JJ, Mcmaster S, Fleming CC, Maule AG, 2010a. Short interfering RNA-mediated gene silencing in Globodera pallida and Meloidogyne incognita infective stage juveniles. International Journal for Parasitology 40, 91-100.

Dalzell JJ, Mcveigh P, Warnock ND, et al., 2011. RNAi Effector Diversity in Nematodes. Plos Neglected Tropical Diseases 5.

The present document has been produced and adopted by the bodies identified above as authors. This task has been carried out exclusively by the author(s) in the context of a contract between the European Food Safety Authority and the authors, awarded following a tender procedure. The present document is published complying with the transparency principle to which the Authority is subject. It may not be considered as an output adopted by the Authority. The European Food Safety Authority reserves its rights, view and position as regards the issues addressed and the conclusions reached in the present document, without prejudice to the rights of the authors. 
Dalzell J], Warnock ND, Stevenson MA, Mousley A, Fleming CC, Maule AG, 2010b. Short interfering RNA-mediated knockdown of drosha and pasha in undifferentiated Meloidogyne incognita eggs leads to irregular growth and embryonic lethality. International Journal for Parasitology 40, 130310.

Deng F, Zhao Z, 2014. influence of catalase gene silencing on the survivability of sitobion avenae. Archives of Insect Biochemistry and Physiology 86, 46-57.

Desai SD, Eu YJ, Whyard S, Currie RW, 2012. Reduction in deformed wing virus infection in larval and adult honey bees (Apis mellifera L.) by double-stranded RNA ingestion. Insect Molecular Biology 21, 446-55.

Ding S-W, 2010. RNA-based antiviral immunity. Nature Reviews Immunology 10, 632-44.

Ding S-W, Voinnet O, 2007. Antiviral immunity directed by small RNAs. Cell 130, 413-26.

Dowling D, Pauli T, Donath A, et al., 2016. Phylogenetic Origin and Diversification of RNAi Pathway Genes in Insects. Genome Biology and Evolution 8, 3784-93.

Dzitoyeva S, Dimitrijevic N, Manev $\mathrm{H}$, 2001. Intra-abdominal injection of double-stranded RNA into anesthetized adult Drosophila triggers RNA interference in the central nervous system. Molecular Psychiatry 6, 665-70.

Dzitoyeva S, Dimitrijevic N, Manev H, 2003. Identification of a novel Drosophila gene, beltless, using injectable embryonic and adult RNA interference (RNAi). Bmc Genomics 4.

Eaton BA, Fetter RD, Davis GW, 2002. Dynactin is necessary for synapse stabilization. Neuron 34, 729-41.

Eichner C, Harasimczuk E, Nilsen F, Grotmol S, Dalvin S, 2015a. Molecular characterisation and functional analysis of LsChi2, a chitinase found in the salmon louse (Lepeophtheirus salmonis salmonis, Kroyer 1838). Experimental parasitology 151, 39-48.

Eichner C, Nilsen F, Grotmol S, Dalvin S, 2014. A method for stable gene knock-down by RNA interference in larvae of the salmon louse (Lepeophtheirus salmonis). Experimental parasitology 140, 44-51.

Eichner C, Øvergárd AC, Nilsen F, Dalvin S, 2015b. Molecular characterization and knock-down of salmon louse (Lepeophtheirus salmonis) prostaglandin E synthase. Experimental parasitology 159, 79-93.

Elzinga DA, De Vos M, Jander G, 2014. Suppression of Plant Defenses by a Myzus persicae (Green Peach Aphid) Salivary Effector Protein. Molecular Plant-Microbe Interactions 27, 747-56.

Fan J, Zhang Y, Francis F, Cheng D, Sun J, Chen J, 2015. Orco mediates olfactory behaviors and winged morph differentiation induced by alarm pheromone in the grain aphid, Sitobion avenae. Insect Biochemistry and Molecular Biology 64, 16-24.

Feinberg EH, Hunter CP, 2003. Transport of dsRNA into cells by the transmembrane protein SID-1. Science 301, 1545-7.

Felix M-A, 2008. RNA interference in nematodes and the chance that favored Sydney Brenner. Journal of Biology 7, 1-5.

Fire A, Xu S, Montgomery MK, Kostas SA, Driver SE, Mello CC, 1998. Potent and specific genetic interference by double-stranded RNA in Caenorhabditis elegans. Nature (London) 391, 806-11.

The present document has been produced and adopted by the bodies identified above as authors. This task has been carried out exclusively by the author(s) in the context of a contract between the European Food Safety Authority and the authors, awarded following a tender procedure. The present document is published complying with the transparency principle to which the Authority is subject. It may not be considered as an output adopted by the Authority. The European Food Safety Authority reserves its rights, view and position as regards the issues addressed and the conclusions reached in the present document, without prejudice to the rights of the authors. 
Flores-Escobar B, Rodriguez-Magadan H, Bravo A, Soberon M, Gomez I, 2013. Differential Role of Manduca sexta Aminopeptidase-N and Alkaline Phosphatase in the Mode of Action of Cry1Aa, Cry1Ab, and Cry1Ac Toxins from Bacillus thuringiensis. Applied and Environmental Microbiology 79, 4543-50.

Fraser AG, Kamath RS, Zipperlen P, Martinez-Campos M, Sohrmann M, Ahringer J, 2000. Functional genomic analysis of C-elegans chromosome I by systematic RNA interference. Nature 408, 325-30.

Galay RL, Hernandez EP, Talactac MR, et al., 2016. Induction of gene silencing in Haemaphysalis longicornis ticks through immersion in double-stranded RNA. Ticks and Tick-Borne Diseases 7, 813-6.

Garbutt JS, Belles X, Richards EH, Reynolds SE, 2013. Persistence of double-stranded RNA in insect hemolymph as a potential determiner of RNA interference success: Evidence from Manduca sexta and Blattella germanica. Journal of Insect Physiology 59, 171-8.

Garbutt JS, Reynolds SE, 2012. Induction of RNA interference genes by double-stranded RNA; implications for susceptibility to RNA interference. Insect Biochemistry and Molecular Biology 42, 621-8.

Geldhof P, Murray L, Couthier A, et al., 2006. Testing the efficacy of RNA interference in Haemonchus contortus. International Journal for Parasitology 36, 801-10.

Gomez I, Flores B, Bravo A, Soberon M, 2015. Bacillus thuringiensis Cry1 AbMod toxin counters tolerance associated with low cadherin expression but not that associated with low alkaline phosphatase expression in Manduca sexta. Peptides 68, 130-3.

Gong H, Umemiya R, Zhou J, et al., 2009. Blocking the secretion of saliva by silencing the HIYkt6 gene in the tick Haemaphysalis longicornis. Insect Biochemistry and Molecular Biology 39, 372-81.

Gong L, Chen Y, Hu Z, Hu M, 2013. Testing Insecticidal Activity of Novel Chemically Synthesized siRNA against Plutella xylostella under Laboratory and Field Conditions. PLoS ONE 8.

Grbić M, Van Leeuwen T, Clark RM, et al., 2011. The genome of Tetranychus urticae reveals herbivorous pest adaptations. Nature 479, 487-92.

Griebler M, Westerlund SA, Hoffmann KH, Meyering-Vos M, 2008. RNA interference with the allatoregulating neuropeptide genes from the fall armyworm Spodoptera frugiperda and its effects on the JH titer in the hemolymph. Journal of Insect Physiology 54, 997-1007.

Guo H, Song X, Wang G, et al., 2014. Plant-Generated Artificial Small RNAs Mediated Aphid Resistance. PLOS ONE 9.

Haegeman A, Bauters L, Kyndt T, Rahman MM, Gheysen G, 2013. Identification of candidate effector genes in the transcriptome of the rice root knot nematode Meloidogyne graminicola. Molecular plant pathology 14, 379-90.

Hao P, Lu C, Ma Y, Xu L, Zhu J, Yu X, 2015. Roles of NIAKTIP in the Growth and Eclosion of the Rice Brown Planthopper, Nilaparvata lugens Stal, as Revealed by RNA Interference. International Journal of Molecular Sciences 16, 22888-903.

Havelda Z, Hornyik C, Válóczi A, Burgyán J, 2005. Defective interfering RNA hinders the activity of a tombusvirus-encoded posttranscriptional gene silencing suppressor. Journal of Virology 79, 450-7.

Hirai M, Terenius O, Li W, Faye I, 2004. Baculovirus and dsRNA induce Hemolin, but no antibacterial activity, in Antheraea pernyi. Insect Molecular Biology 13, 399-405.

Höck J, Meister G, 2008. The Argonaute protein family. Genome Biology 9, 210.

The present document has been produced and adopted by the bodies identified above as authors. This task has been carried out exclusively by the author(s) in the context of a contract between the European Food Safety Authority and the authors, awarded following a tender procedure. The present document is published complying with the transparency principle to which the Authority is subject. It may not be considered as an output adopted by the Authority. The European Food Safety Authority reserves its rights, view and position as regards the issues addressed and the conclusions reached in the present document, without prejudice to the rights of the authors. 
Hoy MA, Waterhouse RM, Wu K, et al., 2016. Genome Sequencing of the Phytoseiid Predatoryte Metaseiulus occidentalis Reveals Completely Atomized Hox Genes and Superdynamic Intron Evolution. Genome Biology and Evolution 8, 1762-75.

Huang G, Allen R, Davis EL, Baum TJ, Hussey RS, 2006. Engineering broad root-knot resistance in transgenic plants by RNAi silencing of a conserved and essential root-knot nematode parasitism gene. Proceedings of the National Academy of Sciences of the United States of America 103, 14302-6.

Hunt JH, Mutti NS, Havukainen H, Henshaw MT, Amdam GV, 2011. Development of an RNA Interference Tool, Characterization of Its Target, and an Ecological Test of Caste Differentiation in the Eusocial Wasp Polistes. PLoS ONE 6.

Hunter W, Ellis J, Engelsdorp DV, et al., 2010. Large-scale field application of RNAi technology reducing Israeli Acute Paralysis Virus disease in honey bees (Apis mellifera, Hymenoptera: Apidae). PLoS pathogens, e1001160.

Hussain M, Abraham AM, Asgari S, 2010. An Ascovirus-Encoded RNase III Autoregulates Its Expression and Suppresses RNA Interference-Mediated Gene Silencing. Journal of Virology 84, 3624-30.

Iwami K, Wang J-Y, Jain R, Mccormack S, Johnson L, 1990. Intestinal ornithine decarboxylase: halflife and regulation by putrescine. American Journal of Physiology-Gastrointestinal and Liver Physiology 258, G308-G15.

Ji Q, Huang C-H, Peng J, Hashmi S, Ye T, Chen Y, 2007. Characterization of STIP, a multi-domain nuclear protein, highly conserved in metazoans, and essential for embryogenesis in Caenorhabditis elegans. Experimental Cell Research 313, 1460-72.

Jin S, Singh ND, Li L, Zhang X, Daniell H, 2015. Engineered chloroplast dsRNA silences cytochrome p450 monooxygenase, V-ATPase and chitin synthase genes in the insect gut and disrupts Helicoverpa armigera larval development and pupation. Plant Biotechnology Journal 13, 435-46.

Joseph S, Gheysen G, Subramaniam K, 2012. RNA interference in Pratylenchus coffeae: Knock down of Pc-pat-10 and Pc-unc-87 impedes migration. Molecular and Biochemical Parasitology 186, 51-9.

Kakumani PK, Ponia SS, Rajgokul KS, et al., 2013. Role of RNA Interference (RNAi) in Dengue Virus Replication and Identification of NS4B as an RNAi Suppressor. Journal of Virology 87, 8870-83.

Kamath RS, Martinez-Campos M, Zipperlen P, Fraser AG, Ahringer J, 2001. Effectiveness of specific RNA-mediated interference through ingested double-stranded RNA in Caenorhabditis elegans. Genome Biology 2.

Kamau LM, Wright HW, Nisbet AJ, Bowman AS, 2013. Development of an RNA-interference procedure for gene knockdown in the poultry red mite, Dermanysus gallinae: studies on histamine releasing factor and Cathepsin-D. African Journal of Biotechnology 12, 1350-6.

Kang JS, Koh YH, Moon YS, Lee SH, 2012. Molecular properties of a venom allergen-like protein suggest a parasitic function in the pinewood nematode Bursaphelenchus xylophilus. International Journal for Parasitology 42, 63-70.

Keene KM, Foy BD, Sanchez-Vargas I, Beaty BJ, Blair CD, Olson KE, 2004. RNA interference acts as a natural antiviral response to O'nyong-nyong virus (Alphavirus; Togaviridae) infection of Anopheles gambiae. Proceedings of the National Academy of Sciences of the United States of America 101, 17240-5.

Kennerdell JR, Carthew RW, 1998. Use of dsRNA-mediated genetic interference to demonstrate that frizzled and frizzled 2 act in the wingless pathway. Cell 95, 1017-26.

The present document has been produced and adopted by the bodies identified above as authors. This task has been carried out exclusively by the author(s) in the context of a contract between the European Food Safety Authority and the authors, awarded following a tender procedure. The present document is published complying with the transparency principle to which the Authority is subject. It may not be considered as an output adopted by the Authority. The European Food Safety Authority reserves its rights, view and position as regards the issues addressed and the conclusions reached in the present document, without prejudice to the rights of the authors. 
Khajuria C, Buschman LL, Chen MS, Muthukrishnan S, Zhu KY, 2010. A gut-specific chitinase gene essential for regulation of chitin content of peritrophic matrix and growth of Ostrinia nubilalis larvae. Insect Biochemistry and Molecular Biology 40, 621-9.

Khan AM, Ashfaq M, Khan AA, Rasool A, Iqbal J, Mansoor S, 2015. Inoculation of Nicotiana tabacum with recombinant potato virus $X$ induces RNA interference in the solenopsis mealybug, Phenacoccus solenopsis Tinsley (Hemiptera: Pseudococcidae). Biotechnology Letters 37, 2083-90.

Khan AM, Ashfaq M, Kiss Z, Khan AA, Mansoor S, Falk BW, 2013. Use of Recombinant Tobacco Mosaic Virus To Achieve RNA Interference in Plants against the Citrus Mealybug, Planococcus citri (Hemiptera: Pseudococcidae). PLoS ONE 8.

Knight M, Miller A, Liu Y, Scaria P, Woodle M, Ittiprasert W, 2011. Polyethyleneimine (PEI) Mediated siRNA Gene Silencing in the Schistosoma mansoni Snail Host, Biomphalaria glabrata. Plos Neglected Tropical Diseases 5.

Kuhar MJ, Joyce AR, 2001. Slow onset of CNS drugs: can changes in protein concentration account for the delay? Trends in Pharmacological Sciences 22, 450-6.

Kumar P, Pandit SS, Baldwin IT, 2012. Tobacco Rattle Virus Vector: A Rapid and Transient Means of Silencing Manduca sexta Genes by Plant Mediated RNA Interference. PLOS ONE 7.

Kumar P, Pandit SS, Steppuhn A, Baldwin IT, 2014. Natural history-driven, plant-mediated RNAibased study reveals CYP6B46's role in a nicotine-mediated antipredator herbivore defense. Proceedings of the National Academy of Sciences of the United States of America 111, 1245-52.

Kurscheid S, Lew-Tabor AE, Valle MR, et al., 2009. Evidence of a tick RNAi pathway by comparative genomics and reverse genetics screen of targets with known loss-of-function phenotypes in Drosophila. Bmc Molecular Biology 10, 26.

Kwon D, Park J, Ashok PA, Lee U, Lee S, 2016. Screening of target genes for RNAi in Tetranychus urticae and RNAi toxicity enhancement by chimeric genes. Pesticide Biochemistry and Physiology 130, 1-7.

Kwon D, Park J, Lee S, 2013. Screening of lethal genes for feeding RNAi by leaf disc-mediated systematic delivery of dsRNA in Tetranychus urticae. Pesticide Biochemistry and Physiology 105, 69-75.

Lai AG, Aboobaker AA, 2017. Comparative genomic analysis of innate immunity reveals novel and conserved components in crustacean food crop species. Bmc Genomics 18, 389.

Leigh O, Kelly C, Praveen R, Indrani K, 2015. Diet-delivery of therapeutic RNA interference in live Escherichia coli against pre-existing Penaeus merguiensis hepandensovirus in Penaeus merguiensis. Aquaculture 437, 360-5.

Li H, Khajuria C, Rangasamy M, et al., 2015a. Long dsRNA but not siRNA initiates RNAi in western corn rootworm larvae and adults. Journal of Applied Entomology 139, 432-45.

Li H, Li WX, Ding SW, 2002. Induction and suppression of RNA silencing by an animal virus. Science 296, 1319-21.

Li X, Dong X, Zou C, Zhang H, 2015b. Endocytic pathway mediates refractoriness of insect Bactrocera dorsalis to RNA interference. Scientific Reports 5.

Li X, Yang L, Jiang S, Fu M, Huang J, Jiang S, 2013. Identification and expression analysis of Dicer2 in black tiger shrimp (Penaeus monodon) responses to immune challenges. Fish \& Shellfish Immunology 35, 1-8.

Li X, Zhang M, Zhang H, 2011. RNA Interference of Four Genes in Adult Bactrocera dorsalis by Feeding Their dsRNAs. PLOS ONE 6.

The present document has been produced and adopted by the bodies identified above as authors. This task has been carried out exclusively by the author(s) in the context of a contract between the European Food Safety Authority and the authors, awarded following a tender procedure. The present document is published complying with the transparency principle to which the Authority is subject. It may not be considered as an output adopted by the Authority. The European Food Safety Authority reserves its rights, view and position as regards the issues addressed and the conclusions reached in the present document, without prejudice to the rights of the authors. 
Liu F, Wang X, Zhao Y, Li Y, Liu Y, Sun J, 2015. Silencing the HaAK Gene by Transgenic PlantMediated RNAi Impairs Larval Growth of Helicoverpa armigera. International Journal of Biological Sciences 11, 67-74.

Liu J, Smagghe G, Swevers L, 2013. Transcriptional response of BmTo119-1 and RNAi machinery genes to exogenous dsRNA in the midgut of Bombyx mori. Journal of Insect Physiology 59, 64654.

Liu J, Swevers L, Iatrou K, Huvenne H, Smagghe G, 2012. Bombyx mori DNA/RNA non-specific nuclease: Expression of isoforms in insect culture cells, subcellular localization and functional assays. Journal of Insect Physiology 58, 1166-76.

Liu QH, Rand TA, Kalidas S, et al., 2003. R2D2, a bridge between the initiation and effector steps of the Drosophila RNAi pathway. Science 301, 1921-5.

Lok JB, 2007. Strongyloides stercoralis: a model for translational research on parasitic nematode biology. WormBook: the online review of C. elegans biology, 1-18.

Lomate PR, Bonning BC, 2016. Distinct properties of proteases and nucleases in the gut, salivary gland and saliva of southern green stink bug, Nezara viridula. Scientific Reports 6, 27587.

Louvet-Vallée S, Kolotuev I, Podbilewicz B, Félix M-A, 2003. Control of vulval competence and centering in the nematode Oscheius sp. 1 CEW1. Genetics 163, 133-46.

Loy JD, Mogler MA, Loy DS, et al., 2012. dsRNA provides sequence-dependent protection against infectious myonecrosis virus in Litopenaeus vannamei. Journal of General Virology 93, 880-8.

Lozano J, Gomez-Orte E, Lee H-J, Belles X, 2012. Super-induction of Dicer-2 expression by alien double-stranded RNAs: an evolutionary ancient response to viral infection? Development Genes and Evolution 222, 229-35.

Lu R, Maduro M, Li F, et al., 2005. Animal virus replication and RNAi-mediated antiviral silencing in Caenorhabditis elegans. Nature 436, 1040-3.

Lu S, Cullen BR, 2004. Adenovirus VA1 noncoding RNA can inhibit small interfering RNA and MicroRNA biogenesis. Journal of Virology 78, 12868-76.

Luo Y, Wang X, Wang X, Yu D, Chen B, Kang L, 2013. Differential responses of migratory locusts to systemic RNA interference via double-stranded RNA injection and feeding. Insect Molecular Biology 22, 574-83.

Ma HB, Lu Q, Liang J, Zhang XY, 2011. Functional analysis of the cellulose gene of the pine wood nematode, Bursaphelenchus xylophilus, using RNA interference. Genetics and Molecular Research $10,1931-41$.

Maeda I, Kohara Y, Yamamoto M, Sugimoto A, 2001. Large-scale analysis of gene function in Caenorhabditis elegans by high-throughput RNAi. Current Biology 11, 171-6.

Mao J, Zeng F, 2012. Feeding-Based RNA Intereference of a Gap Gene Is Lethal to the Pea Aphid, Acyrthosiphon pisum. PLoS ONE 7.

Mao Y, Cai W, Wang J, et al., 2007. Silencing a cotton bollworm P450 monooxygenase gene by plantmediated RNAi impairs larval tolerance of gossypol. Nature Biotechnology 25, 1307-13.

Maori E, Kalev H, Shafir S, Sela I, Garbian Y, 2012. Bidirectional Transfer of RNAi between Honey Bee and Varroa destructor: Varroa Gene Silencing Reduces Varroa Population. Figshare 1.

Maori E, Paldi N, Shafir S, et al., 2009. IAPV, a bee-affecting virus associated with Colony Collapse Disorder can be silenced by dsRNA ingestion. Insect Molecular Biology 18, 55-60.

The present document has been produced and adopted by the bodies identified above as authors. This task has been carried out exclusively by the author(s) in the context of a contract between the European Food Safety Authority and the authors, awarded following a tender procedure. The present document is published complying with the transparency principle to which the Authority is subject. It may not be considered as an output adopted by the Authority. The European Food Safety Authority reserves its rights, view and position as regards the issues addressed and the conclusions reached in the present document, without prejudice to the rights of the authors. 
Marques JT, Kim K, Wu P-H, Alleyne TM, Jafari N, Carthew RW, 2010. Loqs and R2D2 act sequentially in the siRNA pathway in Drosophila. Nature Structural \& Molecular Biology 17, 24-U37.

Marr EJ, Sargison ND, Nisbet AJ, Burgess STG, 2015. Gene silencing by RNA interference in the house dust mite, Dermatophagoides pteronyssinus. Molecular and Cellular Probes 29, 522-6.

Mcewan DL, Weisman AS, Hunter CP, 2012. Uptake of extracellular double-stranded RNA by SID-2. Molecular Cell 47, 746-54.

Mctaggart SJ, Conlon C, Colbourne JK, Blaxter ML, Little TJ, 2009. The components of the Daphnia pulex immune system as revealed by complete genome sequencing. Bmc Genomics 10, 175.

Meleshkevitch EA, Voronov DA, Miller MM, et al., 2013. A novel eukaryotic $\mathrm{Na}+$ methionine selective symporter is essential for mosquito development. Insect Biochemistry and Molecular Biology 43, 755-67.

Miller SC, Brown SJ, Tomoyasu Y, 2008. Larval RNAi in Drosophila? Development Genes and Evolution 218, 505-10.

Miller SC, Miyata K, Brown SJ, Tomoyasu Y, 2012. Dissecting Systemic RNA Interference in the Red Flour Beetle Tribolium castaneum: Parameters Affecting the Efficiency of RNAi. PLoS ONE 7.

Misquitta L, Paterson BM, 1999. Targeted disruption of gene function in Drosophila by RNA interference (RNA-i): A role for nautilus in embryonic somatic muscle formation. Proceedings of the National Academy of Sciences of the United States of America 96, 1451-6.

Misquitta L, Wei $Q$, Paterson BM, 2008. Injection of dsRNA into Drosophila Embryos for RNA Interference (RNAi). CSH protocols 2008, pdb.prot4918-pdb.prot.

Morton DG, Shakes DC, Nugent S, et al., 2002. The Caenorhabditis elegans par-5 gene encodes a 143-3 protein required for cellular asymmetry in the early embryo. Developmental Biology 241, 4758.

Mukherjee K, Campos H, Kolaczkowski B, 2013. Evolution of animal and plant dicers: early parallel duplications and recurrent adaptation of antiviral RNA binding in plants. Molecular Biology and Evolution 30, 627-41.

Murphy KA, Tabuloc CA, Cervantes KR, Chiu JC, 2016. Ingestion of genetically modified yeast symbiont reduces fitness of an insect pest via RNA interference. Scientific Reports 6.

Mutti NS, Park Y, Reese JC, Reeck GR, 2006. RNAi knockdown of a salivary transcript leading to lethality in the pea aphid, Acyrthosiphon pisum. Journal of Insect Science 6.

Nayak A, Berry B, Tassetto M, et al., 2010. Cricket paralysis virus antagonizes Argonaute 2 to modulate antiviral defense in Drosophila. Nature Structural \& Molecular Biology 17, 547-U41.

Niu J, Smagghe G, Coninck DIMD, Nieuwerburgh FV, Deforce D, Meeus I, 2016. In vivo study of Dicer-2-mediated immune response of the small interfering RNA pathway upon systemic infections of virulent and avirulent viruses in Bombus terrestris. Insect Biochemistry and Molecular Biology 70, 127-37.

Nsengimana J, Bauters L, Haegeman A, Gheysen G, 2013. Silencing of Mg-pat-10 and Mg-unc-87 in the plant parasitic nematode Meloidogyne graminicola using siRNAs. Agriculture 3, 567-78.

Nuez I, Felix M-A, 2012. Evolution of Susceptibility to Ingested Double-Stranded RNAs in Caenorhabditis Nematodes. PLoS ONE 7.

Nunes FMF, Simões ZLP, 2009. A non-invasive method for silencing gene transcription in honeybees maintained under natural conditions. Insect Biochemistry and Molecular Biology 39, 157-60.

The present document has been produced and adopted by the bodies identified above as authors. This task has been carried out exclusively by the author(s) in the context of a contract between the European Food Safety Authority and the authors, awarded following a tender procedure. The present document is published complying with the transparency principle to which the Authority is subject. It may not be considered as an output adopted by the Authority. The European Food Safety Authority reserves its rights, view and position as regards the issues addressed and the conclusions reached in the present document, without prejudice to the rights of the authors. 
Obbard DJ, Gordon KH, Buck AH, Jiggins FM, 2009. The evolution of RNAi as a defence against viruses and transposable elements. Philosophical Transactions of the Royal Society of London B: Biological Sciences 364, 99-115.

Obbard DJ, Jiggins FM, Halligan DL, Little TJ, 2006. Natural selection drives extremely rapid evolution in antiviral RNAi genes. Current Biology 16, 580-5.

Ocimati W, Kiggundu A, Bailey A, et al., 2016. Suppression of the ubiquitin E2 gene through RNA interference causes mortality in the banana weevil, Cosmopolites sordidus (Germar). Acta Horticulturae, 181-9.

Okamura K, Balla S, Martin R, Liu N, Lai EC, 2008. Two distinct mechanisms generate endogenous siRNAs from bidirectional transcription in Drosophila melanogaster. Nature Structural \& Molecular Biology 15, 581-90.

Ooi C-P, Haines LR, Southern DM, Lehane MJ, Acosta-Serrano A, 2015. Tsetse GmmSRPN10 Has Anticomplement Activity and Is Important for Successful Establishment of Trypanosome Infections in the Fly Midgut. Plos Neglected Tropical Diseases 9.

Ozawa R, Nishimura O, Yazawa S, Muroi A, Takabayashi J, Arimura G-I, 2012. Temperaturedependent, behavioural, and transcriptional variability of a tritrophic interaction consisting of bean, herbivorous mite, and predator. Molecular Ecology 21, 5624-35.

Pathak KB, Nagy PD, 2009. Defective interfering RNAs: foes of viruses and friends of virologists. Viruses 1, 895-919.

Peng $H$, Peng $D$, Long $H$, et al., 2014. Characterisation and functional importance of beta-1,4endoglucanases from the potato rot nematode, Ditylenchus destructor. Nematology 16, 505-17.

Perrimon N, Ni J-Q, Perkins L, 2010. In vivo RNAi: Today and Tomorrow. Cold Spring Harbor Perspectives in Biology 2.

Piot N, Snoeck S, Vanlede M, Smagghe G, Meeus I, 2015. The Effect of Oral Administration of dsRNA on Viral Replication and Mortality in Bombus terrestris. Viruses-Basel 7, 3172-85.

Pires-Dasilva A, Sommer RJ, 2004. Conservation of the global sex determination gene tra-1 in distantly related nematodes. Genes \& Development 18, 1198-208.

Pitino M, Coleman AD, Maffei ME, Ridout CJ, Hogenhout SA, 2011. Silencing of Aphid Genes by dsRNA Feeding from Plants. PLOS ONE 6.

Pitino M, Hogenhout SA, 2013. Aphid Protein Effectors Promote Aphid Colonization in a Plant SpeciesSpecific Manner. Molecular Plant-Microbe Interactions 26, 130-9.

Pomerantz AF, Hoy MA, 2015. RNAi-mediated knockdown of transformer-2 in the predatory mite Metaseiulus occidentalis via oral delivery of double-stranded RNA. Experimental and Applied Acarology 65, 17-27.

Poreddy S, Mitra S, Schoettner M, et al., 2015. Detoxification of hostplant's chemical defence rather than its anti-predator co-option drives beta-glucosidase-mediated lepidopteran counteradaptation. Nature Communications 6.

Porta H, Jimenez G, Cordoba E, Leon P, Soberon M, Bravo A, 2011. Tobacco plants expressing the Cry1AbMod toxin suppress tolerance to Cry1Ab toxin of Manduca sexta cadherin-silenced larvae. Insect Biochemistry and Molecular Biology 41, 513-9.

Prentice K, Christiaens O, Pertry I, et al., 2017. RNAi-based gene silencing through dsRNA injection or ingestion against the African sweet potato weevil Cylas puncticollis (Coleoptera: Brentidae). Pest Management Science 73, 44-52.

The present document has been produced and adopted by the bodies identified above as authors. This task has been carried out exclusively by the author(s) in the context of a contract between the European Food Safety Authority and the authors, awarded following a tender procedure. The present document is published complying with the transparency principle to which the Authority is subject. It may not be considered as an output adopted by the Authority. The European Food Safety Authority reserves its rights, view and position as regards the issues addressed and the conclusions reached in the present document, without prejudice to the rights of the authors. 
Pridgeon JW, Zhao L, Becnel JJ, Strickman DA, Clark GG, Linthicum KJ, 2008. Topically applied AaeIAP1 double-stranded RNA kills female adults of Aedes aegypti. Journal of Medical Entomology 45, 414-20.

Qiu L, Hou L, Zhang B, et al., 2015. Cadherin is involved in the action of Bacillus thuringiensis toxins Cry1Ac and Cry2Aa in the beet armyworm, Spodoptera exigua. Journal of Invertebrate Pathology 127, 47-53.

Ramesh Kumar D, Elumalai R, Raichur AM, et al., 2016. Development of antiviral gene therapy for Monodon baculovirus using dsRNA loaded chitosan-dextran sulfate nanocapsule delivery system in Penaeus monodon post-larvae. Antiviral research 131, 124-30.

Ratzka C, Gross R, Feldhaar H, 2013. Systemic gene knockdown in Camponotus floridanus workers by feeding of dsRNA. Insectes Sociaux 60, 475-84.

Raza A, Malik HJ, Shafiq M, et al., 2016. RNA Interference based Approach to Down Regulate Osmoregulators of Whitefly (Bemisia tabaci): Potential Technology for the Control of Whitefly. PLOS ONE 11.

Reardon W, Chakrabortee S, Pereira TC, et al., 2010. Expression profiling and cross-species RNA interference (RNAi) of desiccation-induced transcripts in the anhydrobiotic nematode Aphelenchus avenae. Bmc Molecular Biology 11.

Rehman S, Butterbach $\mathrm{P}$, Popeijus $\mathrm{H}$, et al., 2009. Identification and Characterization of the Most Abundant Cellulases in Stylet Secretions from Globodera rostochiensis. Phytopathology 99, 194202.

Robalino J, Bartlett T, Shepard E, et al., 2005. Double-stranded RNA induces sequence-specific antiviral silencing in addition to nonspecific immunity in a marine shrimp: Convergence of RNA interference and innate immunity in the invertebrate antiviral response? Journal of Virology 79, 13561-71.

Saksmerprome V, Thammasorn T, Jitrakorn S, Wongtripop S, Borwornpinyo S, Withyachumnarnkul B, 2013. Using double-stranded RNA for the control of Laem-Singh Virus (LSNV) in Thai P-monodon. Journal of Biotechnology 164, 449-53.

Saleh M-C, Van Rij RP, Hekele A, et al., 2006. The endocytic pathway mediates cell entry of dsRNA to induce RNAi silencing. Nature Cell Biology 8, 793-U19.

Sanitt P, Apiratikul N, Niyomtham N, et al., 2016. Cholesterol-based cationic liposome increases dsRNA protection of yellow head virus infection in Penaeus vannamei. Journal of Biotechnology 228, 95-102.

Sanitt P, Attasart P, Panyim S, 2014. Protection of yellow head virus infection in shrimp by feeding of bacteria expressing dsRNAs. Journal of Biotechnology 179, 26-31.

Sapountzis P, Duport G, Balmand S, et al., 2014. New insight into the RNA interference response against cathepsin-L gene in the pea aphid, Acyrthosiphon pisum: Molting or gut phenotypes specifically induced by injection or feeding treatments. Insect Biochemistry and Molecular Biology 51, 20-32.

Sarathi M, Simon MC, Venkatesan C, Hameed ASS, 2008. Oral administration of bacterially expressed VP28dsRNA to protect Penaeus monodon from white spot syndrome virus. Marine Biotechnology 10, 242-9.

Sarathi M, Simon MC, Venkatesan C, et al., 2010. Efficacy of bacterially expressed dsRNA specific to different structural genes of white spot syndrome virus (WSSV) in protection of shrimp from WSSV infection. Journal of Fish Diseases 33, 603-7.

The present document has been produced and adopted by the bodies identified above as authors. This task has been carried out exclusively by the author(s) in the context of a contract between the European Food Safety Authority and the authors, awarded following a tender procedure. The present document is published complying with the transparency principle to which the Authority is subject. It may not be considered as an output adopted by the Authority. The European Food Safety Authority reserves its rights, view and position as regards the issues addressed and the conclusions reached in the present document, without prejudice to the rights of the authors. 
Schmid A, Schindelholz B, Zinn K, 2002. Combinatorial RNAi: a method for evaluating the functions of gene families in Drosophila. Trends in Neurosciences 25, 71-4.

Schnettler E, Sterken MG, Leung JY, et al., 2012. Noncoding flavivirus RNA displays RNA interference suppressor activity in insect and Mammalian cells. Journal of Virology 86, 13486-500.

Schnettler $E$, Tykalová $H$, Watson $M$, et al., 2014. Induction and suppression of tick cell antiviral RNAi responses by tick-borne flaviviruses. Nucleic Acids Research 42, 9436-46.

Schott DH, Cureton DK, Whelan SP, Hunter CP, 2005. An antiviral role for the RNA interference machinery in Caenorhabditis elegans. Proceedings of the National Academy of Sciences of the United States of America 102, 18420-4.

Schumpert CA, Anderson C, Dudycha JL, Patel RC, 2016. Involvement of Daphnia pulicaria Sir2 in regulating stress response and lifespan. Aging-Us 8, 402-17.

Schumpert CA, Dudycha JL, Patel RC, 2015. Development of an efficient RNA interference method by feeding for the microcrustacean Daphnia. Bmc Biotechnology 15.

Shakesby AJ, Wallace IS, Isaacs HV, Pritchard J, Roberts DM, Douglas AE, 2009. A water-specific aquaporin involved in aphid osmoregulation. Insect Biochemistry and Molecular Biology 39, 1-10.

Shi L, Wei P, Wang X, et al., 2016. Functional Analysis of Esterase TCE2 Gene from Tetranychus cinnabarinus (Boisduval) involved in Acaricide Resistance. Scientific Reports 6.

Shim J, Lee G, Lee S, Lee K, 2015. Oral ingestion of heat shock protein 70 dsRNA is lethal under normal and thermal stress conditions in the sweetpotato whitefly, Bemisia tabaci. Journal of AsiaPacific Entomology 18, 797-800.

Shukla JN, Kalsi M, Sethi A, et al., 2016. Reduced stability and intracellular transport of dsRNA contribute to poor RNAi response in lepidopteran insects. RNA biology 13, 656-69.

Sidahmed A, Abdalla S, Mahmud S, Wilkie B, 2014. Antiviral innate immune response of RNA interference. Journal of Infection in Developing Countries 8, 804-10.

Singh G, Popli S, Hari Y, Malhotra P, Mukherjee S, Bhatnagar RK, 2009. Suppression of RNA silencing by Flock house virus B2 protein is mediated through its interaction with the PAZ domain of Dicer. Faseb Journal 23, 1845-57.

Sivamaruthi BS, Balamurugan K, 2014. Physiological and Immunological Regulations in Caenorhabditis elegans Infected with Salmonella enterica serovar Typhi. Indian Journal of Microbiology 54, 52-8.

Soares CaG, Lima CMR, Dolan MC, Piesman J, Beard CB, Zeidner NS, 2005. Capillary feeding of specific dsRNA induces silencing of the isac gene in nymphal Ixodes scapularis ticks. Insect Molecular Biology 14, 443-52.

Song MH, Huang FZ, Chang GY, Weisblat DA, 2002. Expression and function of an even-skipped homolog in the leech Helobdella robusta. Development 129, 3681-92.

Spit J, Philips A, Wynant N, Santos D, Plaetinck G, Broeck JV, 2017. Knockdown of nuclease activity in the gut enhances RNAi efficiency in the Colorado potato beetle, Leptinotarsa decemlineata, but not in the desert locust, Schistocerca gregaria. Insect biochemistry and molecular biology 81, 103-16.

Su J, Oanh DTH, Lyons RE, et al., 2008. A key gene of the RNA interference pathway in the black tiger shrimp, Penaeus monodon: Identification and functional characterisation of Dicer-1. Fish \& Shellfish Immunology 24, 223-33.

The present document has been produced and adopted by the bodies identified above as authors. This task has been carried out exclusively by the author(s) in the context of a contract between the European Food Safety Authority and the authors, awarded following a tender procedure. The present document is published complying with the transparency principle to which the Authority is subject. It may not be considered as an output adopted by the Authority. The European Food Safety Authority reserves its rights, view and position as regards the issues addressed and the conclusions reached in the present document, without prejudice to the rights of the authors. 
Suzuki T, Nunes MA, España MU, et al., 2017. RNAi-based reverse genetics in the chelicerate model Tetranychus urticae: A comparative analysis of five methods for gene silencing. PLoS ONE 12, e0180654.

Swevers L, Broeck JV, Smagghe G, 2013a. The possible impact of persistent virus infection on the function of the RNAi machinery in insects: a hypothesis. Frontiers in Physiology 4.

Swevers L, Huvenne H, Menschaert G, et al., 2013b. Colorado potato beetle (Coleoptera) gut transcriptome analysis: expression of RNA interference-related genes. Insect Molecular Biology 22, 668-84.

Swevers L, Huvenne H, Smagghe G, Liu J, 2011. Search for Limiting Factors in the RNAi Pathway in Silkmoth Tissues and the Bm5 Cell Line: The RNA-Binding Proteins R2D2 and Translin. Figshare.

Swevers L, Ioannidis K, Kolovou M, et al., 2016. Persistent RNA virus infection of lepidopteran cell lines: Interactions with the RNAi machinery. Journal of Insect Physiology 93-94, 81-93

Tang T, Huang D-W, Zhou C-Q, Li X, Xie Q-J, Liu F-S, 2012a. Molecular cloning and expression patterns of copper/zinc superoxide dismutase and manganese superoxide dismutase in Musca domestica. Gene 505, 211-20.

Tang T, Wu C, Li J, Ren G, Huang D, Liu F, 2012b. Stress-induced HSP70 from Musca domestica plays a functionally significant role in the immune system. Journal of Insect Physiology 58, 1226-34.

Taning CN, Andrade EC, Hunter WB, Christiaens O, Smagghe G, 2016a. Asian Citrus Psyllid RNAi Pathway-RNAi evidence. Scientific Reports 6, 38082.

Taning CNT, Christiaens O, Berkvens N, Casteels H, Maes M, Smagghe G, 2016b. to control Drosophila suzukii: laboratory testing against larval and adult stages. Journal of Pest Science 89, 803-14.

Tassetto M, Kunitomi M, Andino R, 2017. Circulating immune cells mediate a systemic RNAi-based adaptive antiviral response in Drosophila. Cell 169, 314-325

Terenius O, Bettencourt R, Lee SY, Li W, Soderhall K, Faye I, 2007. RNA interference of Hemolin causes depletion of phenoloxidase activity in Hyalophora cecropia. Developmental and Comparative Immunology 31, 571-5.

Terenius O, Papanicolaou A, Garbutt JS, et al., 2011. RNA interference in Lepidoptera: An overview of successful and unsuccessful studies and implications for experimental design. Journal of Insect Physiology 57, 231-45.

Thakur N, Upadhyay SK, Verma PC, Chandrashekar K, Tuli R, Singh PK, 2014. Enhanced Whitefly Resistance in Transgenic Tobacco Plants Expressing Double Stranded RNA of v-ATPase A Gene. PLOS ONE 9.

Tian G, Cheng L, Qi X, et al., 2015a. Transgenic Cotton Plants Expressing Double-stranded RNAs Target HMG-CoA Reductase (HMGR) Gene Inhibits the Growth, Development and Survival of Cotton Bollworms. International Journal of Biological Sciences 11, 1296-305.

Tian H, Yu S, Liu B, et al., 2015b. Molecular cloning of heat shock protein gene HSP90 and effects of abamectin and double-stranded RNA on its expression in Panonychus citri (Trombidiformes: Tetranychidae). Florida Entomologist 98, 37-43.

Tijsterman M, Okihara KL, Thijssen K, Plasterk RHA, 2002. PPW-1, a PAZ/PIWI protein required for efficient germline RNAi, is defective in a natural isolate of C-elegans. Current Biology 12, 1535-40.

Timmons L, Fire A, 1998. Specific interference by ingested dsRNA. Nature (London) 395, 854.

The present document has been produced and adopted by the bodies identified above as authors. This task has been carried out exclusively by the author(s) in the context of a contract between the European Food Safety Authority and the authors, awarded following a tender procedure. The present document is published complying with the transparency principle to which the Authority is subject. It may not be considered as an output adopted by the Authority. The European Food Safety Authority reserves its rights, view and position as regards the issues addressed and the conclusions reached in the present document, without prejudice to the rights of the authors. 
Tomoyasu Y, Miller SC, Tomita S, Schoppmeier M, Grossmann D, Bucher G, 2008. Exploring systemic RNA interference in insects: a genome-wide survey for RNAi genes in Tribolium. Genome Biology 9.

Turner CT, Davy MW, Macdiarmid RM, Plummer KM, Birch NP, Newcomb RD, 2006. RNA interference in the light brown apple moth, Epiphyas postvittana (Walker) induced by double-stranded RNA feeding. Insect Molecular Biology 15, 383-91.

Ulvila J, Parikka M, Kleino A, et al., 2006. Double-stranded RNA is internalized by scavenger receptormediated endocytosis in Drosophila S2 cells. Journal of Biological Chemistry 281, 14370-5.

Upadhyay SK, Chandrashekar K, Nidhi T, et al., 2011. RNA interference for the control of whiteflies (Bemisia tabaci) by oral route. Journal of Biosciences 36, 153-61.

Van Rij RP, Saleh M-C, Berry B, et al., 2006. The RNA silencing endonuclease Argonaute 2 mediates specific antiviral immunity in Drosophila melanogaster. Genes \& Development 20, 2985-95.

Verzijl N, Degroot J, Thorpe SR, et al., 2000. Effect of collagen turnover on the accumulation of advanced glycation end products. Journal of Biological Chemistry 275, 39027-31.

Visser A, Geldhof P, De Maere V, Knox D, Vercruysse J, Claerebout E, 2006. Efficacy and specificity of RNA interference in larval life-stages of Ostertagia ostertagi. Parasitology 133, 777.

Walshe DP, Lehane SM, Lehane MJ, Haines LR, 2009. Prolonged gene knockdown in the tsetse fly Glossina by feeding double stranded RNA. Insect Molecular Biology 18, 11-9.

Wang G, Li N, Zhang L, Zhang L, Zhang Z, Wang Y, 2016a. IGFBP7 is involved in abalone metamorphosis. Aquaculture 451, 377-84.

Wang K, Li Y, Huang X, Wang D-W, Xu C-L, Xie H, 2016b. The cathepsin S cysteine proteinase of the burrowing nematode Radopholus similis is essential for the reproduction and invasion. Cell and Bioscience 6.

Wang $\mathrm{XH}$, Aliyari R, Li WX, et al., 2006. RNA interference directs innate immunity against viruses in adult Drosophila. Science 312, 452-4.

Wheeler D, Darby BJ, Todd TC, Herman MA, 2012. Several Grassland Soil Nematode Species Are Insensitive to RNA-Mediated Interference. Journal of Nematology 44, 92-101.

Whyard S, Singh AD, Wong S, 2009. Ingested double-stranded RNAs can act as species-specific insecticides. Insect Biochemistry and Molecular Biology 39, 824-32.

Wilkins C, Dishongh R, Moore SC, Whitt MA, Chow M, Machaca K, 2005. RNA interference is an antiviral defence mechanism in Caenorhabditis elegans. Nature 436, 1044-7.

Willert K, Logan CY, Arora A, Fish M, Nusse R, 1999. A Drosophila Axin homolog, Daxin, inhibits Wnt signaling. Development 126, 4165-73.

Winston WM, Sutherlin M, Wright AJ, Feinberg EH, Hunter CP, 2007. Caenorhabditis elegans SID-2 is required for environmental RNA interference. Proceedings of the National Academy of Sciences of the United States of America 104, 10565-70.

Wu K, Hoy MA, 2014a. Clathrin Heavy Chain Is Important for Viability, Oviposition, Embryogenesis and, Possibly, Systemic RNAi Response in the Predatory Mite Metaseiulus occidentalis. PLOS ONE 9.

Wu K, Hoy MA, 2014b. Oral delivery of double-stranded RNA induces prolonged and systemic gene knockdown in Metaseiulus occidentalis only after feeding on Tetranychus urticae. Experimental and Applied Acarology 63, 171-87.

The present document has been produced and adopted by the bodies identified above as authors. This task has been carried out exclusively by the author(s) in the context of a contract between the European Food Safety Authority and the authors, awarded following a tender procedure. The present document is published complying with the transparency principle to which the Authority is subject. It may not be considered as an output adopted by the Authority. The European Food Safety Authority reserves its rights, view and position as regards the issues addressed and the conclusions reached in the present document, without prejudice to the rights of the authors. 
Wu K, Hoy MA, 2015. Cloning and Functional Characterization of Two BTB Genes in the Predatory Mite Metaseiulus occidentalis. PLoS ONE 10.

Wu P, Li M, Wang X, et al., 2009. Differentially expressed genes in the midgut of silkworm infected with cytoplasmic polyhedrosis virus. African Journal of Biotechnology 8.

Wuriyanghan H, Falk BW, 2013. RNA Interference towards the Potato Psyllid, Bactericera cockerelli, Is Induced in Plants Infected with Recombinant Tobacco mosaic virus (TMV). PLOS ONE 8.

Wuriyanghan H, Rosa C, Falk BW, 2011. Oral Delivery of Double-Stranded RNAs and siRNAs Induces RNAi Effects in the Potato/Tomato Psyllid, Bactericerca cockerelli. PLoS ONE 6.

Wynant N, Santos D, Vanden Broeck J, 2014a. Biological Mechanisms Determining the Success of RNA Interference in Insects. In: Jeon K, ed. International Review of Cell and Molecular Biology, Vol 312. 139-67. (International Review of Cell and Molecular Biology; vol. 312.)

Wynant N, Santos D, Verdonck R, Spit J, Van Wielendaele P, Vanden Broeck J, 2014b. Identification, functional characterization and phylogenetic analysis of double stranded RNA degrading enzymes present in the gut of the desert locust, Schistocerca gregaria. Insect Biochemistry and Molecular Biology 46, 1-8.

Wynant N, Verlinden $H$, Breugelmans B, Simonet G, Broeck JV, 2012. Tissue-dependence and sensitivity of the systemic RNA interference response in the desert locust, Schistocerca gregaria. Insect Biochemistry and Molecular Biology 42, 911-7.

Yadav BC, Karuppannan V, Kuppuswamy S, 2006. Host-generated double stranded RNA induces RNAi in plant-parasitic nematodes and protects the host from infection. Molecular and Biochemical Parasitology 148, 219-22.

Yao X, Wang L, Song L, et al., 2010. A Dicer-1 gene from white shrimp Litopenaeus vannamei: Expression pattern in the processes of immune response and larval development. Fish \& Shellfish Immunology 29, 565-70.

Yi X, Zhao H, Dong X, Wang P, Hu M, Zhong G, 2013. BdorCSP2 Is Important for Antifeed and Oviposition-Deterring Activities Induced by Rhodojaponin-III against Bactrocera dorsalis. PLoS ONE 8.

Zha W, Peng X, Chen R, Du B, Zhu L, He G, 2011. Knockdown of Midgut Genes by dsRNA-Transgenic Plant-Mediated RNA Interference in the Hemipteran Insect Nilaparvata lugens. PLoS ONE 6.

Zhang G, He L-S, Wong YH, Yu L, Qian P-Y, 2015a. siRNA transfection in larvae of the barnacle Amphibalanus amphitrite. Journal of Experimental Biology 218, 2505-9.

Zhang J, Khan SA, Hasse C, Ruf S, Heckel DG, Bock R, 2015b. Full crop protection from an insect pest by expression of long double-stranded RNAs in plastids. Science 347, 991-4.

Zhang L-L, Liang G-M, Gao X-W, Cao G-C, Guo Y-Y, 2015c. Characterization and functional analysis of beta-1,3-galactosyltransferase involved in Cry1Ac resistance from Helicoverpa armigera (Hubner). Journal of Integrative Agriculture 14, 337-46.

Zhang L, Shang Q, Lu Y, Zhao Q, Gao X, 2015d. A transferrin gene associated with development and 2-tridecanone tolerance in Helicoverpa armigera. Insect Molecular Biology 24, 155-66.

Zhang $M$, Zhou $Y$, Wang $H$, et al., 2013. Identifying potential RNAi targets in grain aphid (Sitobion avenae $F$.) based on transcriptome profiling of its alimentary canal after feeding on wheat plants. Bmc Genomics 14, 1-15.

Zhang X, Zhang J, Zhu KY, 2010. Chitosan/double-stranded RNA nanoparticle-mediated RNA interference to silence chitin synthase genes through larval feeding in the African malaria mosquito (Anopheles gambiae). Insect Molecular Biology 19, 683-93.

The present document has been produced and adopted by the bodies identified above as authors. This task has been carried out exclusively by the author(s) in the context of a contract between the European Food Safety Authority and the authors, awarded following a tender procedure. The present document is published complying with the transparency principle to which the Authority is subject. It may not be considered as an output adopted by the Authority. The European Food Safety Authority reserves its rights, view and position as regards the issues addressed and the conclusions reached in the present document, without prejudice to the rights of the authors. 
Zhang Y, Deng F, Fan Y, Zhao Z, 2016. Effects of carboxylesterase gene silence on wheat aphid Sitobion avenae (Fabricius). Journal of Asia-Pacific Entomology 19, 341-5.

Zhang Y, Zhang S, Kulye M, et al., 2012. Silencing of molt-regulating transcription factor gene, CiHR3, affects growth and development of sugarcane stem borer, Chilo infuscatellus. Journal of Insect Science (Madison) 12, (9 August 2012).

Zhao Y, Yang G, Wang-Pruski G, You M, 2008. Phyllotreta striolata (Coleoptera: Chrysomelidae): Arginine kinase cloning and RNAi-based pest control. European Journal of Entomology 105, 81522.

Zheng M, Long H, Zhao Y, et al., 2015. RNA-Seq Based Identification of Candidate Parasitism Genes of Cereal Cyst Nematode (Heterodera avenae) during Incompatible Infection to Aegilops variabilis. PLOS ONE 10.

Zhou XG, Oi FM, Scharf ME, 2006. Social exploitation of hexamerin: RNAi reveals a major casteregulatory factor in termites. Proceedings of the National Academy of Sciences of the United States of America 103, 4499-504.

Zhu J, Dong Y-C, Li P, Niu C-Y, 2016. The effect of silencing 20E biosynthesis relative genes by feeding bacterially expressed dsRNA on the larval development of Chilo suppressalis. Scientific Reports 6.

Zhu J, Liu S, Ma Y, et al., 2012. Improvement of pest resistance in transgenic tobacco plants expressing dsRNA of an insect-associated gene EcR. PLoS ONE 7, e38572.

The present document has been produced and adopted by the bodies identified above as authors. This task has been carried out exclusively by the author(s) in the context of a contract between the European Food Safety Authority and the authors, awarded following a tender procedure. The present document is published complying with the transparency principle to which the Authority is subject. It may not be considered as an output adopted by the Authority. The European Food Safety Authority reserves its rights, view and position as regards the issues addressed and the conclusions reached in the present document, without prejudice to the rights of the authors. 


\subsection{Off-target, non-target and unintended effects of RNAi-based GM plants (Task 5)}

\subsubsection{Introduction}

Food and energy turnover is one of the pillars of the functioning of every ecosystem, the way organic matter flows through an ecosystem is influenced by the organisms within the system. In agroecosystems, as in all other terrestrial ecosystems, plants constitute the primary source of energy supporting food webs on which other functional groups are sustained. Different orders of consumers in grazing or detritus food webs constitute the animal diversity which allows the provision of ecological services in agriculture (Table 14).

Table 14: Examples of functional groups of invertebrate species and ecosystem services provided to agriculture

\begin{tabular}{|c|c|c|}
\hline $\begin{array}{l}\text { Functional } \\
\text { group }\end{array}$ & Examples & $\begin{array}{l}\text { Examples of ecosystem } \\
\text { functions and services }\end{array}$ \\
\hline Herbivores & $\begin{array}{l}\text { Sap/cell feeders: e.g., Acarina, Homoptera: Aphidoidea, } \\
\text { Aleyrodidae, Cicadellidae; Thysanoptera: Thripidae. } \\
\text { Leaf feeders: e.g., Coleoptera: Chrysomelidae, } \\
\text { Curculionidae, Lepidoptera: Noctuidae, Pieridae, } \\
\text { Tortricidae; Gasteropoda: snails, slugs } \\
\text { Leaf miners: e.g., Diptera: Agromyzidae, Lepidoptera: } \\
\text { Gelechiidae } \\
\text { Root feeders: e.g., juveniles Hemiptera: Heteroptera, } \\
\text { Diptera, Symphyla } \\
\text { Seed feeders: e.g., Coleoptera: Carabidae, } \\
\text { Hymenoptera: Formicidae }\end{array}$ & $\begin{array}{l}\text { Nutrient cycling } \\
\text { (as prey/hosts for natural } \\
\text { enemies) }\end{array}$ \\
\hline Predators & $\begin{array}{l}\text { Thysanoptera (e.g., Aeolothripidae); } \\
\text { Heteroptera (e.g., Anthocoridae, Miridae, Nabidae); } \\
\text { Neuroptera (e.g., Chrysopidae, Hemerobidae); } \\
\text { Coleoptera (e.g., Coccinellidae, Carabidae, } \\
\text { Staphilinidae); Diptera (e.g., Syrphidae, } \\
\text { Cecidomyiidae); } \\
\text { Araneae; Acarina (e.g., Phytoseiidae) } \\
\text { Nematoda }\end{array}$ & $\begin{array}{l}\text { Population regulation } \\
\text { (natural control of arthropod } \\
\text { pests) }\end{array}$ \\
\hline Parasitoids & Diptera (e.g., Tachinidae); Hymenoptera Parasitica & $\begin{array}{l}\text { Population regulation (natural } \\
\text { control of arthropod pests) }\end{array}$ \\
\hline $\begin{array}{l}\text { Pollinators, } \\
\text { pollen feeders }\end{array}$ & $\begin{array}{l}\text { Diptera: Syrphidae; Hymenoptera: Apidae; Coleoptera: } \\
\text { Coccinellidae }\end{array}$ & Pollination, honey production \\
\hline Decomposers & $\begin{array}{l}\text { Nematoda, Collembola, Acarina, Isopoda, Haplotaxida: } \\
\text { Lumbricidae }\end{array}$ & $\begin{array}{l}\text { Decomposing litter, soil } \\
\text { structure and fertility, } \\
\text { Nutrient cycling (as prey/hosts } \\
\text { for natural enemies) }\end{array}$ \\
\hline
\end{tabular}

GM plants expressing invertebrate resistance traits could introduce potentially harmful novel metabolites into existing food webs (e.g., Cry toxins, proteinase inhibitors, lectins). Therefore, higher order consumers might possibly be adversely affected (Lövei and Arpaia, 2005). All living organisms that are not meant to be affected by newly expressed compounds in GM plants, and that can be potentially exposed, directly or indirectly, to the GM plant and/or its products are considered as "nontarget" organisms (Arpaia, 2010). NTOs could be affected either directly, by feeding on plant parts or preys containing RNAi-triggering molecules, or indirectly, by the reliance on a suboptimal diet due to the changed quality of the plants and/or the herbivore preys (Andow et al., 2006).

The present document has been produced and adopted by the bodies identified above as authors. This task has been carried out exclusively by the author(s) in the context of a contract between the European Food Safety Authority and the authors, awarded following a tender procedure. The present document is published complying with the transparency principle to which the Authority is subject. It may not be considered as an output adopted by the Authority. The European Food Safety Authority reserves its rights, view and position as regards the issues addressed and the conclusions reached in the present document, without prejudice to the rights of the authors. 
Another possible concern is that unintentional changes in expression of some metabolites in GM plants or changes in plant composition and structure may affect ecological interactions. Arpaia et al. (2017) showed that genetic modification can also induce a range of unintended effects on plant metabolism and overall phenotype, though their biological relevance needs to be evaluated case by case.

In this section, we will present the literature specifically dealing with off target and non-target effects of GM plants expressing sRNAs primarily targeted at invertebrate genes. Similar as in Section 5.3, we have first performed an assessment of relevant papers, dealing specifically with RNAi off-target effects and effects on NTOs, in the initial list of 4,612 oral exposure studies. In this search, we found a total of 41 studies which dealt specifically with these topics (Appendix E).

\subsubsection{Food chain effects}

Traits of individual plants influence interactions between plants, herbivores and natural enemies (Price et al., 1980). These traits may be molecular (e.g., toxins, antifeedants, sRNAs) or physical (e.g., pubescence, toughness). Effects are often mediated between different trophic levels, i.e., sublethal effects such as delayed development, induced by plants may have consequences on the activity of natural enemies of the herbivores (Johnson and Gould, 1992). Therefore, any change in plant composition due to genetic modification may induce effects at different trophic levels.

Herbivores may acquire plant constitutive compounds and herbivores possess a number of mechanisms to deal with these compounds (e.g., degradation, sequestration, excretion). As a result, some products derived from plants, or their metabolites, are present in the body of herbivores.

Some invertebrate species show specialization with regard to the feeding sites they occupy on their host plants. Since plant compounds, including RNA molecules, are expressed at different levels in plant tissues, the feeding habits of the herbivores determine their exposure to plant compounds.

However, the occurrence of natural defensive compounds is usually concentrated in the regions where their presence may increase the fitness of the plant when attacked. Thus, knowledge of the expression of sRNA as well as endogenous compounds in different plant parts is important for determining exposure both in herbivores and in species at higher trophic levels exposed to these herbivores.

When a significant change in plant composition occurs in a host plant, a "counter-adaptation" is needed for the herbivore to continue feeding on this plant. Changes in host plants can consequently affect organisms at the second trophic level and may also produce changes in their quality as hosts/preys for natural enemies due to the additional presence of novel compounds in their body. In turn, natural enemies which normally feed on their hosts/preys with a "familiar" chemical composition will have to cope with new metabolites (intact or partly digested) which were acquired during herbivory. Finally, compounds originated from the host plant may interact with the herbivore's constitutive compounds representing a new challenge to the natural enemies' metabolism.

The possible outcomes of the changes in trophic interactions along food webs are therefore to be determined on a case by case basis.

\subsubsection{RNA-expressing genetically modified plants}

The silencing effect of the siRNA is highly dependent on the active metabolism of the target organisms. Furthermore, since basically any gene of interest could be targeted with RNAi, some completely new modes of action are involved in the process. For instance, GM maize MON 87411 expresses, among other traits, a dsRNA targeting the DvSnf7 gene, a gene involved in vacuolar sorting at cellular level, a mechanism which has not been previously targeted with any insecticidal compound or GM plant.

The present document has been produced and adopted by the bodies identified above as authors. This task has been carried out exclusively by the author(s) in the context of a contract between the European Food Safety Authority and the authors, awarded following a tender procedure. The present document is published complying with the transparency principle to which the Authority is subject. It may not be considered as an output adopted by the Authority. The European Food Safety Authority reserves its rights, view and position as regards the issues addressed and the conclusions reached in the present document, without prejudice to the rights of the authors. 
Several mechanisms triggered by dsRNA exposure were suggested to possibly produce unintended effects. Here, we divide them into two main categories, namely (1) sequence-dependent mechanisms such as off-target gene silencing, silencing genes in NTOs and (2) sequence-independent mechanisms such as immune stimulation and saturation of the RNAi machinery (Lundgren and Duan, 2013). Offtarget effects in the target species may not necessarily be a safety issue, but we discuss the literature on both off-target effects and unintended effects on NTOs, since both rely on the same mechanisms, which is related to the specificity of RNAi, more specifically the minimum homology between an siRNA and the target mRNA sequence leading to successful gene silencing.

\subsubsection{Sequence-dependent mechanisms}

Within a eukaryotic cell, long dsRNA is cleaved by the enzyme Dicer into siRNAs, which are about 21 nucleotides in length. The siRNAs, in conjunction with the RISC, scan the cell's mRNA molecules until a complementary match is found. Once recognized, the target mRNA is cleaved by the Argonaute in the RISC with consequent silencing of the corresponding gene. Thus, a highly sequence-specific gene silencing can be achieved in some invertebrates by feeding dsRNAs (Baum et al., 2007; Swevers and Smagghe, 2012; Whyard et al., 2009). Due to this mechanism of action, dsRNA-expressing plants targeting invertebrate herbivores are expected to be a highly selective means of pest control, since a high level of specificity can be achieved with the appropriate selection of target genes and target sequences within the gene of interest. Depending on the selected sequence, either a single species can be efficiently targeted or, in cases of more conserved sequences, a broader specificity can be achieved (Runo et al., 2011). Unfortunately, little fundamental biochemical information in invertebrates is available on the necessary homology between siRNA and mRNA to cause effective RNAi gene silencing. However, a number of studies conducted in invertebrates give some indication on the necessary level of homology. In this section, we review the available data on the necessary sequence homology to incite RNAi silencing, the potential for off-target effects and studies investigating the potential for unintended effects on NTOs.

\subsubsection{Target specificity of dsRNA}

There is no consensus among scientists on the number of nucleotides that must match the target sequence identically or on the allowed number and type of mismatches in invertebrates. Although dsRNA expressed in crop plants as means of pest control has been claimed to have a high degree of specificity (Dillin, 2003; Petrick et al., 2013; Whyard et al., 2009) other studies have shown that siRNAs can silence unintended genes (Davidson and McCray, 2011). However, fundamental and direct evidence of the siRNA homology necessary to cause gene silencing in invertebrates not found in our literature search. In mammals, several studies have shown that mismatches between siRNA and target mRNA can be allowed while still leading to successful gene silencing (Huang et al., 2009; Jackson et al., 2003; Jackson et al., 2006; Schwarz et al., 2006). Further research is required to determine whether the same issues apply to invertebrates. It is also important to note that, when using long dsRNA, the effector molecules after Dicer processing of the dsRNAs are very diverse and the concentration of any single siRNA is much lower than the original dsRNA concentration. Therefore, a single siRNA able to cause gene silencing does not necessarily lead to a strong gene silencing or any phenotypical effects.

Whyard et al. (2009) obtained V-ATPase specific dsRNA for four different insect species from different orders: the fruit fly $D$. melanogaster, the flour beetle $T$. castaneum, the pea aphid $A$. pisum, and the tobacco hornworm M. sexta. When individuals of these species were fed with dsRNA-containing diets in laboratory experiments, high levels of mortality where achieved only when species-specific dsRNA was provided in the diets. In contrast, dsRNA targeting the homologous gene in other species did not produce adverse effects. Moreover, this study also investigated whether specificity between species belonging to the same genus (Drosophila) could be achieved. To this purpose, dsRNAs specific for the gamma-tubulin gene in D. melanogaster, D. sechellia, D. yakubu and D. pseudoobscura were

The present document has been produced and adopted by the bodies identified above as authors. This task has been carried out exclusively by the author(s) in the context of a contract between the European Food Safety Authority and the authors, awarded following a tender procedure. The present document is published complying with the transparency principle to which the Authority is subject. It may not be considered as an output adopted by the Authority. The European Food Safety Authority reserves its rights, view and position as regards the issues addressed and the conclusions reached in the present document, without prejudice to the rights of the authors. 
designed and fed to these fruitflies. The results confirmed that selectivity between these species was possible (Whyard et al., 2009).

Likewise, Baum et al. (2007) tested the potential for species selectivity based on nucleotide-sequence identity with the WCR, D. virgifera virgifera and the CPB, L. decemlineata using dsRNA that targets the gene encoding the V-ATPase subunit $A$ and $E$ for each species. The V-ATPase subunit A target sequences from CPB and WCR share $83 \%$ nucleotide-sequence identity whereas the V-ATPase subunit $E$ target sequences from these organisms share $79 \%$ nucleotide-sequence identity. Feeding both WCR and CPB with the heterospecific dsRNA that targeted V-ATPase subunits A and E, caused mortality in both species. However, such response was expected given the multiple 21 nt shared sequence over the targeted portion of the gene for these two species.

Bachman et al. (2013) characterized the spectrum of insecticidal activity of a $240 \mathrm{nt}$ dsRNA targeting the Snf7 ortholog in the WCR. They first performed a similar study to the one of Baum et al. (2007), feeding heterospecific dsRNA to CPB and WCR. In contrast to the dsRNA targeting the v-ATPase subunits, the dsSnf7 was species-specific in causing mortality. Analysis of sequence homology showed that the longest match between both homologous sequences was only $14 \mathrm{nt}$. The authors then selected and tested insects of 10 different families and four different orders (i.e., Hemiptera, Hymenoptera, Lepidoptera and Coleoptera) based upon their phylogenetic relatedness to WCR and measured potential lethal and sublethal effects in continuous feeding diet bioassays with DvSnf7 dsRNA. Bioassay results demonstrated that the spectrum of activity for DvSnf7 was narrow and activity was only evident in a group of beetles within the Galerucinae subfamily of Chrysomelidae (>90\% identity with WCR Snf7 $240 \mathrm{nt}$ ). A shared sequence length of $\geq 20$ nt seemed required for efficacy against WCR (containing 221 potential 21-nt matches) and all orthologs susceptible for gene silencing by DvSnf7 contained at least three 21 nt matches with the DvSnf7 sequence.

Zhang et al. (2015) used a lepidopteran pest, the Asian corn borer $O$. furnacalis, as a target species. In order to investigate whether the dsRNA of a gene has species-specific or broad-spectrum activity on Lepidoptera, the authors identified a methionine-rich storage protein gene (OfSP) in the species' genome. dsRNA for the three functional domains of the gene were synthesized and were tested on the larvae of the target species and another lepidopteran, $H$. armigera. The dsRNA targeting the C-terminal domain, which was sprayed on the larvae, caused high mortality rates in both insects, whereas those targeting the M-segment and $\mathrm{N}$-terminal domains only caused high mortality in the Asian corn borer. Sequence analysis revealed one perfect $21 \mathrm{nt}$ match and four $20 \mathrm{nt}$ matches between the dsRNA and the homologous gene in $H$. armigera. Furthermore, several $19 \mathrm{nt}$ matches were found between this dsRNA and several other genes in $H$. armigera belonging to the hexameric storage protein family (Zhang et al., 2015).

\subsubsection{Off-target effects}

The phenomenon of RNAi off-target effects (Birmingham et al., 2006) was described for the first time in eggs of $M$. incognita harvested from infected tobacco lines expressing dsRNA targeting proteases. Non-target proteases genes were up-regulated when the target gene was knocked-down, suggesting the presence of an innate response to compensate for the lack of some proteases, though their upregulation gives no significant effects on nematode development (Antonino de Souza Júnior et al., 2013).

Off-target effects are commonly related to the siRNA sequence itself and most often arise from partial complementarily of the sense or antisense strands to an unintended target within an organism (Jackson et al., 2006). In particular, these effects appear to be related to partial sequence homology between the "seed region" (positions 2-7 or 2-8) of the siRNA and the 3 ' untranslated region of messenger RNA transcribed from a non-target gene, though different conclusions were reached by (Chen et al., 2015).

The present document has been produced and adopted by the bodies identified above as authors. This task has been carried out exclusively by the author(s) in the context of a contract between the European Food Safety Authority and the authors, awarded following a tender procedure. The present document is published complying with the transparency principle to which the Authority is subject. It may not be considered as an output adopted by the Authority. The European Food Safety Authority reserves its rights, view and position as regards the issues addressed and the conclusions reached in the present document, without prejudice to the rights of the authors. 
Given the small sizes of siRNAs, it is not surprising that off-target binding sites are quite common in different organisms (Qiu et al., 2005). Substantial sequence complementarity is needed to trigger offtarget gene effects, but siRNAs containing some mismatches may still effectively trigger silencing (Jackson et al., 2006). The allowance for some mismatches could therefore increase the potential for off-target gene silencing effects.

Sequence dependent off-target effects were found in insects by Kulkarni et al. (2006). Based on genome-wide screenings they confirmed experimentally that dsRNAs containing $\geq 19$ nt perfect matches lead to efficient knockdown of a cross-hybridizing transcript. Even though the use of long dsRNAs in D. melanogaster, where Dicer-mediated processing produces small RNAs inside cells, has been thought to reduce the probability of off-target effects, similar results were obtained by Ma et al. (2006). They conducted genome-wide RNAi screen for novel components of wingless signal transduction and showed that, even in the absence of candidate genes for positively acting wingless pathway components, off-target effects mediated by short homology stretches within long dsRNAs are prevalent in $D$. melanogaster.

Jarosch and Moritz (2012) worked on honeybees as a model system. Bees were tested by injecting three different dsRNAs: two obtained from the honeybee transcriptome (dsGPDH and dsVG) and one targeting a marker gene not present in the honeybee genome (dsGFP). The authors analyzed the gene expression of four non-target genes coding for proteins that are involved in different physiological processes. The genes selected lacked similarity with the dsRNA injected and were: AmSID-I coding for the production of a transmembrane channel protein, amATF-2 a gene regulating transcription factors and stress proteins, amDHAP-AT involved in lipid metabolism and amCPR a cytochrome P450 reductase. All these proteins are commonly used as stress biomarkers. After treatment with three dsRNAs in two abdominal tissues (fat bodies and ovaries), the authors evaluated the tissue-specific responses of the dsRNA. The different dsRNA-off-target gene combinations showed altered transcript abundances after the treatment. All three dsRNAs, specifically designed to have no sequence homology longer than 20 bp with any gene in the honeybee genome, showed at least one unspecific off-target knockdown. However, the authors, considered the observed effects as non- sequence specific off-target effects and indicated the similarity of the metabolic function of the genes as a possible explanation for their results. The authors did not find similar effects when larvae of the studied non-target were injected with the dsRNA solvent, so they concluded that the observations were not the result of a wounding or septic reaction in response to injections. Other possible stressing factors, including injections, have been indicated as possible causes of stimulation of the immune system (see Section 5.4.5.2).

Off-target effects were shown in experiments with honeybees using dsRNA targeting gfp (Nunes et al., 2013). The use of dsRNA as an exogenous control for RNAi testing in arthropods is a wellestablished technique (Mutti et al., 2006; Sim et al., 2012; Tang et al., 2010). The GFP gene does not exist in the honeybee genome and it has therefore also been used as negative control in experiments with this species (Jarosch and Moritz, 2011; Kamakura, 2011; Nunes et al., 2013). Although dsGFP is not expected to trigger an RNAi response in bees, Nunes et al. (2013) report that phenotypical effects in pupal pigmentation and larval development have been observed in RNAi screens using dsGFP as a control. The authors fed honeybee larvae with diets containing dsGFP and checked the individuals at different time points through their development as worker bees. Three different experiments were conducted using different concentrations of dsGFP. Gene expression of the individuals under treatment and their respective negative control bees was determined through microarray analysis. A large number of genes (almost 10\% of the whole genome) appeared to be upregulated or downregulated in bees treated in the larval phase, while a significantly lower number of activated genes appeared after treatment of adults. However, only five genes appeared in the list of differentially expressed genes in each of the different experiments, suggesting that the expression of most genes is changed due to a sequence-unspecific effect. Expression changes appear to be the result of both direct off-target effects and indirect downstream secondary effects; indeed, there were

The present document has been produced and adopted by the bodies identified above as authors. This task has been carried out exclusively by the author(s) in the context of a contract between the European Food Safety Authority and the authors, awarded following a tender procedure. The present document is published complying with the transparency principle to which the Authority is subject. It may not be considered as an output adopted by the Authority. The European Food Safety Authority reserves its rights, view and position as regards the issues addressed and the conclusions reached in the present document, without prejudice to the rights of the authors. 
several instances of sequence similarity between putative siRNAs generated from the dsGFP construct and genes whose expression levels were altered. Thus siRNA-induced silencing can generate specific and non-specific effects on an organism (Davidson and McCray, 2011).

Zhou et al. (2014) conducted a study in C. elegans and showed that nuclear Ago NRDE-3 protein associates with off target siRNAs in C. elegans following administration of exogenous RNAi. These findings supported the idea that the nuclear RNAi pathway is a primary source of exogenous and endogenous off-targeting effects in this species and suggest that off-target silencing is more than a mere biochemical limitation of the RNAi machinery but rather a genetically programmed aspect of the RNAi.

\subsubsection{Non-target effects}

The possible exposure of NTOs to dsRNA expressed in plants is discussed in Section 5.2. In this section, we review the available information on the unintended and off-target effects of RNAi in invertebrate species other than the primary intended target organisms.

Phylogenetic relatedness to the target species and a high degree of homologous overlap between at least one siRNA and the gene sequence are considered to be two important requisites for RNAi effects (Bachman et al., 2013). Baum et al. (2007) reported that V-ATPase dsRNA targeted against the WCR, $D$. virgifera virgifera, proved to be also effective on the $C P B, L$. decemlineata, and noticed a sequence similarity of $83 \%$ of the $V$-ATPase $A$ between the two species. More specifically, the CPB V-ATPase A dsRNA yielded an $\mathrm{LC}_{50}$ value of $5.2 \mathrm{ng} / \mathrm{cm}^{2}$ in the CPB bioassay, whereas the orthologous WCR dsRNA yielded a $\mathrm{LC}_{50}$ value $452 \mathrm{ng} / \mathrm{cm}^{2}$, a greater than ten-fold difference in activity that could be attributed to divergence in target sequence.

Zhu et al. (2012) found that H. armigera ECR dsRNA, targeting a receptor complex of the steroid hormone 20-hydroxyecdysone, expressed in transgenic tobacco plants was also effective against another lepidopteran pest, S. exigua, due to the high similarity in the nucleotide sequences $(89 \%)$ of the two ECR genes. In both species mortality levels of $40-50 \%$ were detected during bioassays with GM tobacco plants.

Pan et al. (2017) developed an in vivo toxicity assay to examine the impacts of ingested dsRNAs targeting the $V$-ATPase $A$ gene on the larvae of the monarch butterfly $D$. plexippus. The full-length $v$ ATPase A cDNAs from the target pest, the WCR, D. virgifera virgifera, (Coleoptera) and the nontarget $D$. plexippus were respectively cloned. To start from a 'worst case scenario', they opted for a 400 bp dsRNA located in a highly conserved region of a highly conserved gene ( $v$-ATPase A). The $V$ ATPase $A$ gene is highly conserved among holometabolous insects and, when analysing DNA sequences from data sets, the authors argued that Coleoptera were sister groups to Lepidoptera and Diptera and more phylogenetically distant from Hymenoptera and other insect orders. The sequence alignment of the $V$-ATPase $A$ gene from $D$. plexippus and $D$. $v$. virgifera enabled identification of a highly conserved region of $400 \mathrm{bp}$ which showed $77 \%$ identity between the two species. The bioinformatic analysis showed that 19-25 nt contiguous sequence matches between the two insect species were present. However, no significant differences in the survival of $D$. plexippus larvae across treatments were observed. The development time from the $1^{\text {st }}$ instar to the adult was not affected by the treatments, though some differences appeared during each larval stage.

Although $D$. plexippus and $D$. virgifera virgifera shared nearly $80 \%$ sequence similarity within the selected $400 \mathrm{nt}$ region of V-ATPase A, the lack of 21-mer matches between target and non-target insect species might explain the results of the bioassays. In addition, it must be remembered that silencing activity of dsRNA in Lepidoptera is very variable among families due to several factors such as non-target species, targeted tissue, delivery methods, dsRNA uptake, dsRNA degradation and gene function (Terenius et al., 2011) (reviewed in Section 5.3). The two above mentioned studies were based on molecular analyses, and there are other cases where adverse effects on NTOs have been identified.

The present document has been produced and adopted by the bodies identified above as authors. This task has been carried out exclusively by the author(s) in the context of a contract between the European Food Safety Authority and the authors, awarded following a tender procedure. The present document is published complying with the transparency principle to which the Authority is subject. It may not be considered as an output adopted by the Authority. The European Food Safety Authority reserves its rights, view and position as regards the issues addressed and the conclusions reached in the present document, without prejudice to the rights of the authors. 
Chen et al. (2015) investigated the effects of dsRNA targeting rpl19 gene from Bactrocera dorsalis on non-target insects which are normally found in the same environment (Citrus cultivations) of the target species, by feeding the dsRNA to the congeneric species Bactrocera minax, the honeybee A. mellifera and the parasitoid wasp Diachasmimorpha longicaudata. Two different dsRNA were produced targeting two different regions of the cloned gene. Both dsRNA extracts were fed to the chosen NTOs in a series of laboratory bioassays, and subsequent gene silencing was measured with RT-PCR. The authors performed a homology search to reveal shared sequence in $r p / 19$ gene between target and non-target species. The expression levels of $r p / 19$ gene in the non-target insects were down-regulated by the $B$. dorsalis rp/19 dsRNA, with the exception of honeybee. The maximum effect (approximately $90 \%$ down-regulation) was obtained on B. minax, but significant effects were also obtained on the hymenopteran $D$. longicaudata (approximately $40 \%$ ) which shared $72 \%$ sequence homology with $B$. dorsalis. A sequence identity of $69 \%$ is shared between $B$. dorsalis and A. mellifera, however there was no apparent down-regulation of the $r p / 19$ gene on the latter. By contrast, the dsRNA targeting the $3^{\prime}$ region did not induce effects on gene expression on any of the non-target species studied.

Pan et al. (2016) chose the collembolan Sinella curviseta as a non-target species to study possible effects of dsRNA targeting the $V$-ATPase A. The dsRNA was constructed in order to simulate a worstcase scenario, since the region with the highest homology ( $85 \%$ in a region 400 bp-long) between the gene of $D$. virgifera virgifera and $S$. curviseta was selected to prepare dsRNA. Several 19- through 23-mer matches were present in the targeted region of the V-ATPase A gene of the two species. Dietary RNAi toxicity assays were conducted and several measurement endpoints were used to determine effects on the collembolan, both at the molecular and phenotypical level. No significant degradation of $V$-ATPase $A$ mRNA was observed. Similarly, there were no significant differences in adult survival rate, adult body length, fecundity and hatching rate. Larvae fed dsRNA developed faster compared to the control, however the biological significance of this difference is not clear. Vélez et al. (2016) evaluated the insecticidal activity of a known lethal dsRNA target for $D$. virgifera, the $V$ ATPase $A$ in larvae and adult honeybees. A $400 \mathrm{nt} V$-ATPase- $A$ dsRNA was designed based on the region of highest sequence similarity between the target and the non-target species. The dsRNA was tested via oral ingestion for specificity against both $D$. virgifera virgifera and $A$. mellifera. Larval mortality occurred only when species-specific dsRNA was provided to insects during bioassays. In bioassays with adults the relative expression of the $V$-ATPase- $A$ in $A$. mellifera was visible up to 48 hours while it was not visible in adults collected at $96 \mathrm{~h}$, suggesting that the reduced gene expression observed at 48 hours for $A m V$-ATPase- $A$ dsRNA was transient. Overall the results indicate that honey bee larval development, adult eclosion and adult survival were unaffected by both $D$. virgifera virgifera as well as $A$. mellifera dsRNA, suggesting that honey bees are insensitive to $V$ ATPase- $A$ dsRNA. The lack of response suggests that the activity spectrum does not only depend on the sequence specificity, but also on the ability of the organism to respond to RNAi. An overview of studies providing information on the relationship between sequence homology and RNAi silencing is given in Table 15.

The present document has been produced and adopted by the bodies identified above as authors. This task has been carried out exclusively by the author(s) in the context of a contract between the European Food Safety Authority and the authors, awarded following a tender procedure. The present document is published complying with the transparency principle to which the Authority is subject. It may not be considered as an output adopted by the Authority. The European Food Safety Authority reserves its rights, view and position as regards the issues addressed and the conclusions reached in the present document, without prejudice to the rights of the authors. 
Table 15: Overview of studies providing information on RNAi silencing effects in non-target species and sequence homology between the dsRNA and the homologous sequence in the non-target species

\begin{tabular}{|c|c|c|c|c|c|c|c|}
\hline Non-target species & Target species & Target gene & $\begin{array}{l}\text { Measurement } \\
\text { endpoint }\end{array}$ & $\begin{array}{l}\text { Sequence } \\
\text { homology }\end{array}$ & Sequence overlap & Effect on nto & Reference \\
\hline $\begin{array}{l}\text { Leptinotarsa } \\
\text { decemlineata } \\
\text { (Coleoptera: } \\
\text { Chrysomelidae) }\end{array}$ & $\begin{array}{l}\text { Diabrotica virgifera } \\
\text { virgifera (Coleoptera: } \\
\text { Chrysomelidae) }\end{array}$ & $\begin{array}{l}\text { V-ATPase A } \\
\text { V-ATPase E }\end{array}$ & Mortality & $79 \%$ & - & $\begin{array}{l}\text { Mortality (10-fold } \\
\text { less toxic) }\end{array}$ & Baum et al. 2007 \\
\hline $\begin{array}{l}\text { Spodoptera exigua } \\
\text { (Lepidoptera: } \\
\text { Noctuidae) }\end{array}$ & $\begin{array}{l}\text { Helicoverpa armigera } \\
\text { (Lepidoptera: } \\
\text { Noctuidae) }\end{array}$ & EcR & Mortality & $89 \%$ & - & Mortality & Zhu et al. 2012 \\
\hline $\begin{array}{l}\text { Danaus plexippus } \\
\text { (Lepidoptera: } \\
\text { Nymphalidae) }\end{array}$ & $\begin{array}{l}\text { Diabrotica virgifera } \\
\text { virgifera (Coleoptera: } \\
\text { Chrysomelidae) }\end{array}$ & V-ATPase A & $\begin{array}{l}\text { Mortality, } \\
\text { development }\end{array}$ & $77 \%$ & $\begin{array}{l}\text { Twelve } 19-\text {, seven } \\
20-, \text { three } 21-\text {, two } \\
22-\text {, one } 23- \\
\text { contiguous matches }\end{array}$ & No effects & Pan et al. 2017 \\
\hline $\begin{array}{l}\text { Bactrocera minax } \\
\text { (Diptera: } \\
\text { Tephritidae) }\end{array}$ & $\begin{array}{l}\text { Bactrocera dorsalis } \\
\text { (Diptera: } \\
\text { Tephritidae) }\end{array}$ & rpl19 & Gene silencing & $93 \%$ & $\begin{array}{l}\text { Two 19-21 bp } \\
\text { overlap }\end{array}$ & $\begin{array}{l}50-70 \% \text { reduction of } \\
\text { gene expression }\end{array}$ & Chen et al. 2015 \\
\hline $\begin{array}{l}\text { Apis mellifera } \\
\text { (Hymenoptera: } \\
\text { Apidae) }\end{array}$ & & & & $69 \%$ & - & No effects & \\
\hline $\begin{array}{l}\text { Diachasmimorpha } \\
\text { longicaudata } \\
\text { (Hymenoptera: } \\
\text { Braconidae) }\end{array}$ & & & & $72 \%$ & One $19-21 b p$ & $40 \%$ reduction & \\
\hline $\begin{array}{l}\text { Sinella curviseta } \\
\text { (Collembola: } \\
\text { Entomobryidae) }\end{array}$ & $\begin{array}{l}\text { Diabrotica virgifera } \\
\text { virgifera (Coleoptera: } \\
\text { Chrysomelidae) }\end{array}$ & V-ATPase A & $\begin{array}{l}\text { Gene silencing, } \\
\text { survival rate, body } \\
\text { length, fecundity, } \\
\text { hatching rate }\end{array}$ & $85 \%$ & $\begin{array}{l}\text { Twelve 19-, seven } \\
20-, \text { three } 21-, \text { two } \\
22-, \text { and one } 23-n t \\
\text { contiguous matches }\end{array}$ & No effects & Pan et al. 2016 \\
\hline $\begin{array}{l}\text { Apis mellifera } \\
\text { (Hymenoptera: } \\
\text { Apidae) }\end{array}$ & $\begin{array}{l}\text { Diabrotica virgifera } \\
\text { virgifera (Coleoptera: } \\
\text { Chrysomelidae) }\end{array}$ & V-ATPase A & $\begin{array}{l}\text { Development, } \\
\text { survival }\end{array}$ & High similarity & $20-, 24-n t$ & No effects & Vélez et al. 2016 \\
\hline
\end{tabular}

The present document has been produced and adopted by the bodies identified above as authors. This task has been carried out exclusively by the author(s) in the context of a contract between the European Food Safety Authority and the authors, awarded following a tender procedure. The present document is published complying with the transparency principle to which the Authority is subject. It may not be considered as an output adopted by the Authority. The European Food Safety Authority reserves its rights, view and position as regards the issues addressed and the conclusions reached in the present document, without prejudice to the rights of the authors. 


\section{Non-target studies on maize MON 87411}

The most extensively studied case of GM plant-expressing dsRNA so far, relates to the characterization of the GM maize MON 87411, for which a non-regulated status has been recently declared by the US Department of Agriculture $^{3}$ (https://www.aphis.usda.gov/brs/aphisdocs/13_29001p_det.pdf). The applicant conducted an ERA of this maize which included an evaluation of impacts on NTOs (Ahmad et al., 2016; Bachman et al., 2016).

The potential for non-target effects of DvSnf7 dsRNA was tested under worst-case laboratory conditions in several of NTOs, including the predators (Coleomegilla maculata, Poecilus chalcites, Aleochara bilineata, C. carnea, Orius insidiosus), parasitoids (Pediobius foveolatus), pollinators ( $A$. mellifera), soil biota (Eisenia andrei, Folsomia candida) as well as aquatic and terrestrial vertebrate species (Bachman et al., 2016). A number of measurement endpoints were used to assess the potential for non-target effects, including mortality and possible indicators of sub-lethal effects (e.g., development time, adult biomass, adult emergence, fertility, fecundity).

The authors quantified the DvSnf7 dsRNA expression levels in different tissue types collected from maize MON 87411 plants across different sites, using the validated QuantiGene assay (Armstrong et al., 2013) and calculated the expression levels in micrograms of RNA per gram of fresh or dry weight tissue (Table 16).

Table 16: DvSnf7 RNA expression levels in different tissue type from MON 87411. Modified from Bachman et al. (2016)

\begin{tabular}{lll}
\hline Tissue type & Developmental stage & Range $\boldsymbol{\mu g} / \mathbf{g}$ \\
\hline Pollen (fwt) & VT-R1 & $0.056 \times 10^{-3}-0.224 \times 10^{-3}$ \\
Leaf (fwt) & V14-R1 & $5.40 \times 10^{-3}-33.8 \times 10^{-3}$ \\
Root (fwt) & V3-V4 & $1.74 \times 10^{-3}-8.00 \times 10^{-3}$ \\
Whole Plant (dwt) & V6-V8 & $33.0 \times 10^{-3}-106 \times 10^{-3}$ \\
Grain (dwt) & R6 & $0.056 \times 10^{-3}-0.175 \times 10^{-3}$ \\
\hline
\end{tabular}

fwt $=$ fresh weight; $d w t=$ dry weight

The DvSnf7 dsRNA maximum expression levels found in these studies were then used to determine the MEEC for testing the surrogate species of NTOs chosen. For all species tested, no statistically significant effects from ingestion of or exposure to DvSnf7 RNA were detected when compared to the control. A synthesis of the experiment conducted is shown in Table 17.

${ }^{3}$ Available at: https://www.aphis.usda.gov/brs/aphisdocs/13_29001p_det.pdf

The present document has been produced and adopted by the bodies identified above as authors. This task has been carried out exclusively by the author(s) in the context of a contract between the European Food Safety Authority and the authors, awarded following a tender procedure. The present document is published complying with the transparency principle to which the Authority is subject. It may not be considered as an output adopted by the Authority. The European Food Safety Authority reserves its rights, view and position as regards the issues addressed and the conclusions reached in the present document, without prejudice to the rights of the authors. 
Table 17: List of laboratory bioassays conducted on invertebrate non-target organisms for the characterization of maize MON 87411 (Ahmad et al., 2016; Bachman et al., 2016).

\begin{tabular}{lll}
\hline Organism & Functional role & Measurement endpoint \\
\hline Apis mellifera & Pollinator & Larval survival and development, worker survival \\
Coleomegilla maculata & Biocontrol agent & Survival, growth and development \\
Aleochara bilineata & Herbivore & Adult survival and reproduction \\
Poecilus chalcites & Biocontrol agent & Survival, growth and development \\
Chrysoperla carnea & Biocontrol agent & Adult survival and reproduction \\
Orius insidiosus & Biocontrol agent & Survival, growth and development \\
Pediobius foveolatus & Biocontrol agent & Adult survival \\
Eisenia andrei & Nutrient cycling & Survival and body weight \\
Folsomia candida & Nutrient cycling & Survival and reproduction \\
\hline
\end{tabular}

Bioinformatic analyses were conducted to evaluate whether the NTO species tested have sufficient genomic match to the DvSnf7 sequence. In silico evaluation with available genomes and transcriptomes did not identify any 21-nucleotide contiguous matches for any of the species.

Ahmad et al. (2016) evaluated the potential impact of maize MON 87411 on non-target arthropods in field experiments. Field trials were conducted at 14 sites in the U.S.A., Argentina and Brazil providing geographic and environmental diversity within maize production areas. Abundance, measured with visual observations and the use of sticky traps, and damage assessment (for pest species) were chosen as endpoints. Twenty arthropod taxa were abundant enough to allow statistical analyses, and nine of them occurred in at least two of the three regions. Across the 20 taxa analyzed, no statistically significant differences in abundance were detected between MON 87411 and the conventional control for 123 of the 128 individual-site comparisons (96.1\%). For the nine widely distributed taxa, no statistically significant differences in abundance were detected between MON 87411 and the conventional control.

\subsubsection{Trophic chain effects}

While several non-target species have been assayed for possible nonspecific RNA interference, only a handful studies have performed trophic experiments which actually demonstrated movement of dsRNA along trophic chains.

Garbian et al. (2012) provided adult honeybees with a diet containing a dsRNA targeting gfp, used as a marker gene. Successively, individuals of the parasitic mite $V$. destructor were introduced into the bee colonies and allowed to feed on them. To test for bidirectional horizontal transfer, some of the mites were then removed after three days from the dsRNA-carrying bees and introduced into a container with untreated bees. DsGFP was detected in RNA extracts of parasitized bees that had not consumed dsRNA. After demonstrating the cross-species, reciprocal exchange of dsRNA between honeybees and mites, the same experiments were repeated with dsRNA aimed at silencing Varroa genes. The authors designed a number of dsRNA targeting fundamental housekeeping genes whose silencing was expected to harm the Varroa mites. The authors achieved a decrease in the mite population of over $60 \%$ when a mixture of dsRNA was fed to bees, demonstrating that dsRNA maintained its biological activity after transferring across species. Cedeño et al. (2015) similarly used this system to target $V$. destructor and also the deformed wing virus infecting bees.

The present document has been produced and adopted by the bodies identified above as authors. This task has been carried out exclusively by the author(s) in the context of a contract between the European Food Safety Authority and the authors, awarded following a tender procedure. The present document is published complying with the transparency principle to which the Authority is subject. It may not be considered as an output adopted by the Authority. The European Food Safety Authority reserves its rights, view and position as regards the issues addressed and the conclusions reached in the present document, without prejudice to the rights of the authors. 


\subsubsection{Sequence-independent mechanisms}

\subsubsection{Saturation of the RNAi machinery}

In the RNAi pathway, high levels of siRNAs could saturate the protein RISC complex (Lundgren and Duan, 2013), as there is a limited number of RNAi effectors, such as Dicer enzymes and RISCs, present within a cell. The saturation process can consequently temporarily inhibit cellular use of RNAi (Jackson and Linsley, 2010; Khan et al., 2009) and compromise some of its natural functions. For example, it could lead to lowering the efficiency of endogenous gene regulation (Agrawal et al., 2003; Dillin, 2003) and it could lead to reduced defenses against viral infection (Dillin, 2003). On the other hand, under realistic exposure conditions, it is not clear whether the oral dose would be sufficient to affect the RNAi machinery of exposed NTOs.

This process of saturation is better documented with a type of siRNA that targets a specific place on the mRNA named small hairpin RNA (shRNA) although it is also known to occur with siRNA (Jackson and Linsley, 2010). In vitro and in vivo shRNA transfection studies implied that one such factor, shared by the shRNA/miRNA pathways and readily saturated, is the nuclear karyopherin exportin- 5 (Grimm et al., 2006). So far however, this phenomenon has not been reported in invertebrate species.

\subsubsection{Immune stimulation}

In invertebrates such as insects and nematodes, RNAi developed evolutionarily as a natural defense mechanism against viruses (Gammon and Mello, 2015; Karlikow et al., 2014). Recently it was found that the injection in the blood stream of small fragments (fewer than $30 \mathrm{nt}$ ) of nanocarrier-formulated siRNA (to facilitate cellular uptake) could activate the mammalian innate immune system via a toll-like receptor (TLR) pathway which recognized and responded to the sequence, length, and structure of siRNAs (Robbins et al., 2009). It is unclear how the immune system of other organisms responds to exogenous administered small RNA (Lundgren and Duan, 2013) and, despite similarities in the innate immune response of insects and mammals (Lundgren and Jurat-Fuentes, 2012), dsRNA-induced innate immune response has been rarely reported in invertebrates (Dong et al., 2009). Furthermore, it is also unclear whether such stimulation of the immune response could lead to adverse effects on the fitness of invertebrate species.

Recent studies described a non-sequence-specific immune response to dsRNA in honeybees, and a reduction of virus titer in virus-infected adult bees (Brutscher et al., 2017; Flenniken and Andino, 2013). Non-sequence-specific dsRNA has also been shown to reduce virus abundance and affect gene expression in bumble bees (Niu et al., 2016; Piot et al., 2015). This immune stimulation effect is not restricted to bees. Non-specific dsRNA-triggered antiviral response has also been reported in the Chinese oak silk moth Antheraea pernyi (Hirai et al., 2004) and in some crustacean species (Dong et al., 2009; Labreuche et al., 2010; Robalino et al., 2005; Robalino et al., 2007; Robalino et al., 2004). Overall, these findings indicate that an invertebrate immune system can recognize dsRNA as a virusassociated molecular pattern, and therefore activate an antiviral response (Robalino et al., 2004).

In several studies, it has been reported that dsRNA causes upregulation of the RNAi machinery components. For example, dsRNA-injection experiments in the tobacco hornworm $M$. sexta, revealed that dicer-2 mRNA levels and, to a lesser extent, argonaute- 2 mRNA levels were elevated following injection in a specific and dose-dependent manner (Garbutt and Reynolds, 2012). An increase of Dicer-2 mRNA level was also observed in $B$. germanica after treatment with a nucleopolyhedrovirus dsRNA (Lozano et al., 2012).

Studies on other taxa, e.g., shrimps and fishes, reported increased levels of dicer-2 mRNA in response to challenge with dsRNA (Chen et al., 2011b; Su et al., 2009).

The present document has been produced and adopted by the bodies identified above as authors. This task has been carried out exclusively by the author(s) in the context of a contract between the European Food Safety Authority and the authors, awarded following a tender procedure. The present document is published complying with the transparency principle to which the Authority is subject. It may not be considered as an output adopted by the Authority. The European Food Safety Authority reserves its rights, view and position as regards the issues addressed and the conclusions reached in the present document, without prejudice to the rights of the authors. 
Some components of the innate immune response might also react to virus-specific dsRNA (Kingsolver et al., 2013). For example Paradkar et al. (2012) reported the activation of different (nonRNAi) innate immune pathways through Dicer-2.

In the fruit fly D. melanogaster Deddouche et al. (2008) reported the upregulation of the Vago gene after infection by the alphavirus Sindbis virus (SINV) and by a Drosophila C virus (DCV), while a third RNA virus, the nodavirus flock house virus (FHV) did not induce Vago expression. In a similar study by Paradkar et al. (2012) it has been shown that the West Nile virus (WNV) dsRNA was able to stimulate Vago production in Culex quinquefasciatus cells, while the bluetongue virus dsRNA did not cause Vago expression to increase. The induction of this gene was dependent on Dicer- 2 but not by other RNAi pathway components (Deddouche et al., 2008). These outcomes suggest an RNAiindependent signaling mechanism for Dicer-2 and a cross-talk between the RNAi and Jak-STAT (Janus kinase (JAK) and Signal Transducer and Activator of Transcription (STAT) pathways (Kingsolver et al., 2013).

Although the exact immune response pathway is still unclear, it seems that non-specific dsRNA is recognized as a PAMP (Pathogen-Associated Molecular Pattern) triggering the anti-viral defense (Liu et al., 2013; Piot et al., 2015).

Dicer proteins, particularly Dicer-2 in Drosophila and mosquitoes, are recognized as the main PRR (Pattern Recognition Receptor) involved in recognition of virus-derived dsRNA and initiating not only the RNAi response, but also the Jak-STAT response (Deddouche et al., 2008; Ding, 2010; Paradkar et al., 2012).

\subsubsection{Resistance mechanisms}

As for most means of pest control, the genetic plasticity of pests, their short life cycle and multiple number of generations favour the insurgence of new pathogenic strains. Possible resistance development mechanisms to RNAi have been proposed. For example, nuclease upregulation or dsRNA uptake malfunction, processing and systemic spread could stop the initiation and spread of RNAi response. RNAi resistance in mammalian cell cultures is known to occur and many RNAi viruses escape RNAi-mediated suppression through mutation of the target region, by encoding viral suppressors, or by cellular factors such as ADAR1 (adenosine deaminases acting on RNA), which is responsible for editing-mediated RNA resistance (Zheng et al., 2005).

While development of resistance to RNAi has not been documented in invertebrates so far, reports of RNAi (phenotypic) different effects and efficacy among insects strains (Kitzmann et al., 2013) give indications that such mechanism may arise. A recent study looking into the geographic variation in RNAi sensitivity in the migratory locust Locusta migratoria, showed that most individuals from two of the analyzed strains were sensitive to injection of different dsRNA, whereas those from two other strains were resistant (Sugahara et al., 2017). Furthermore, selection of dsRNA sensitive-individuals resulted in an increase in RNAi sensitivity in the following generation and the reciprocal crosses between a sensitive and a resistant strain resulted in F1 generations whose response to RNAi was comparable to that of the resistant strain, suggesting that the resistant phenotype might be dominant (Sugahara et al., 2017).

\subsubsection{Conclusions}

The availability of GM plants-incorporating dsRNA has triggered discussions about their risk assessment and the possible adaptations needed for the current ERA frameworks (U.S. Environmental Protection Agency 2014; EFSA 2014). Particularly, the issue of the possible effects on NTOs is confronted with new challenges due to the lack of familiarity with the new modes of action of the RNA interfering mechanisms. At first sight, RNAi technology looks promising due to its potential to be highly species-specific. However, potential unintended off-target and non-target effects and knowledge gaps discussed in this report should be taken into account. The systematic literature

The present document has been produced and adopted by the bodies identified above as authors. This task has been carried out exclusively by the author(s) in the context of a contract between the European Food Safety Authority and the authors, awarded following a tender procedure. The present document is published complying with the transparency principle to which the Authority is subject. It may not be considered as an output adopted by the Authority. The European Food Safety Authority reserves its rights, view and position as regards the issues addressed and the conclusions reached in the present document, without prejudice to the rights of the authors. 
search confirmed that, to date, only a few systems have been specifically investigated for these impacts.

Several publications reported studies of off-target effects and effects on NTOs linked to interference triggered by dsRNA against DvSnf7 or V-ATPase.

The impacts of DvSnf7 expressed by GM maize event MON 87411 on NTOs and certain surrogate species representative of the main ecological groups in agro-ecosystems, have revealed no unintended effects in both in vitro procedures and field studies.

Several studies have also used dsRNA targeting different subunits of the $V$-ATPase gene. In a recent symposium on "Modern Biotechnology in Integrated Crop Management", Haller et al. ${ }^{4}$ described experiments conducted with dsRNA targeting the $V$-ATPase $A$ of $D$. virgifera virgifera and two ladybird species, Coccinella septempunctata and Adalia bipunctata. Both ladybirds were sensitive to dietary RNAi when ingesting their specific dsRNA, with $C$. septempunctata being much more sensitive than $A$. bipunctata. When assays where conducted with the dsRNA targeting $D$. virgifera virgifera $V$ ATPase $A$, adverse effects were detected in the two ladybird species (prolonged developmental time for $A$. bipunctata and significantly reduced survival rate for $C$. septempunctata). The results were supported by bioinformatic analyses that revealed a higher number of possible $21 \mathrm{nt}$ matches of the targeted dsRNA sequence with the V-ATPase $A$ of $C$. septempunctata (34 matches) compared to that of $A$. bipunctata (six matches).

This and other studies have identified an active silencing on NTOs in species taxonomically related to the target organism (i.e., Chrysomelidae and Coccinellidae) in some cases and, in one case, effects were also observed on a species belonging to a different insect order than the target (i.e., Coleoptera and Hymenoptera).

The likelihood of off-target effects has been suggested to be linked to the sequence similarity of the siRNA to any sequence in the genome of the NTO. In addition, some authors indicated that a certain number of mismatches in the siRNA sequence is allowed and silencing effects can still occur in such cases. Other sequence-independent mechanisms might also cause off-target effects such as similarity of functions of some genes, whose RNA might be similarly affected. In some cases, the use of dsRNA targeting genes absent from the genome of the tested species, caused expressional changes in a range of genes. Thus, the accurate design of the dsRNA to induce interference does not exclude the possibility of off-target and/or non-target effects. On the other hand, the question arises whether the presence of one $21 \mathrm{nt}$ match siRNA in a pool of many different siRNAs after Dicer-processing of the long dsRNA would result in a silencing response strong enough to cause any significant effects.

The support of bioinformatics in this respect could be very valuable, but the limited availability of insect genomic sequences, the possible silencing in the presence of mismatches between the target and the siRNA sequences, and the possibility of sequence-unrelated off-target effects indicate the fundamental role of bioassays in assessing the actual activity spectrum of dsRNA.

Possible non-sequence dependent mechanisms leading to unintended effects on non-target invertebrates orally exposed to dsRNA have been hypothesized (e.g., immune-stimulation, saturation of the dsRNA machinery), however supporting experimental data are not available to date. Similarly, potential mechanisms which could lead to the onset of resistance in target organisms exist and will need to be duly considered.

${ }^{4}$ Available at: http://www.eigmo.info/sites/default/files/Book\%20of\%20abstracts_final.pdf

The present document has been produced and adopted by the bodies identified above as authors. This task has been carried out exclusively by the author(s) in the context of a contract between the European Food Safety Authority and the authors, awarded following a tender procedure. The present document is published complying with the transparency principle to which the Authority is subject. It may not be considered as an output adopted by the Authority. The European Food Safety Authority reserves its rights, view and position as regards the issues addressed and the conclusions reached in the present document, without prejudice to the rights of the authors. 


\subsubsection{References}

Agrawal N, Dasaradhi PVN, Mohmmed A, Malhotra P, Bhatnagar RK and Mukherjee SK, 2003. RNA Interference: Biology, Mechanism, and Applications. Microbiology and Molecular Biology Reviews $67,657-685$.

Ahmad A, Negri I, Oliveira W, et al., 2016. Transportable data from non-target arthropod field studies for the environmental risk assessment of genetically modified maize expressing an insecticidal double-stranded RNA. Transgenic Research 25, 1-17.

Andow DA, Lovei GL, Arpaia S, 2006. Ecological risk assessment for Bt crops. Nature Biotechnology, 24, 749-51.

Antonino De Souza Júnior JD, Ramos Coelho R, Tristan Lourenço I, et al., 2013. KnockingDown Meloidogyne incognita Proteases by Plant-Delivered dsRNA Has Negative Pleiotropic Effect on Nematode Vigor. PloS one 8, e85364.

Armstrong TA, Chen $\mathrm{H}$, Ziegler TE, et al., 2013. Quantification of Transgene-Derived Double-Stranded RNA in Plants Using the QuantiGene Nucleic Acid Detection Platform. Journal of Agricultural and Food Chemistry 61, 12557-64.

Arpaia S, 2010. Genetically modified plants and "non-target" organisms: analysing the functioning of the agro-ecosystem. Collection of Biosafety review 5, 12-80.

Arpaia S, Birch ANE, Kiss J, et al., 2017. Assessing environmental impacts of genetically modified plants on non-target organisms: The relevance of in planta studies. Science of The Total Environment 583, 123-32.

Bachman PM, Bolognesi R, Moar WJ, et al., 2013. Characterization of the spectrum of insecticidal activity of a double-stranded RNA with targeted activity against Western Corn Rootworm (Diabrotica virgifera virgifera LeConte). Transgenic Research 22, 1207-22.

Bachman PM, Huizinga KM, Jensen PD, et al., 2016. Ecological risk assessment for DvSnf7 RNA: A plant-incorporated protectant with targeted activity against western corn rootworm. Regulatory Toxicology and Pharmacology 81, 77-88.

Baum JA, Bogaert T, Clinton W, et al., 2007. Control of coleopteran insect pests through RNA interference. Nat Biotech 25, 1322-6.

Birmingham A, Anderson EM, Reynolds A, et al., 2006. 3[prime] UTR seed matches, but not overall identity, are associated with RNAi off-targets. Nat Meth 3, 199-204.

Brutscher LM, Daughenbaugh KF, Flenniken ML, 2017. Virus and dsRNA-triggered transcriptional responses reveal key components of honey bee antiviral defense. Scientific Reports 7, 6448.

Cedeño AG, Nakaya P, Niz J, Sciocco-Cap A, Salvador R, 2015. Double-stranded RNA synthesized in bacteria can be transferred to bee and varroa tissues. Journal of Apicultural Research 54, 99-100.

Chen A, Zheng W, Zheng W, Zhang H, 2015. The effects of RNA interference targeting Bactrocera dorsalis ds-Bdrpl19 on the gene expression of rpl19 in non-target insects. Ecotoxicology 24, 595603.

Chen $\mathrm{Y}-\mathrm{H}$, Jia X-T, Zhao L, et al., 2011. Identification and functional characterization of Dicer2 and five single VWC domain proteins of Litopenaeus vannamei. Developmental \& Comparative Immunology 35, 661-71.

Davidson BL, Mccray PB, 2011. Current prospects for RNA interference-based therapies. Nat Rev Genet 12, 329-40.

The present document has been produced and adopted by the bodies identified above as authors. This task has been carried out exclusively by the author(s) in the context of a contract between the European Food Safety Authority and the authors, awarded following a tender procedure. The present document is published complying with the transparency principle to which the Authority is subject. It may not be considered as an output adopted by the Authority. The European Food Safety Authority reserves its rights, view and position as regards the issues addressed and the conclusions reached in the present document, without prejudice to the rights of the authors. 
Deddouche S, Matt N, Budd A, et al., 2008. The DExD/H-box helicase Dicer-2 mediates the induction of antiviral activity in drosophila. 9, 1425.

Dillin A, 2003. The specifics of small interfering RNA specificity. Proceedings of the National Academy of Sciences of the United States of America 100, 6289-91.

Ding S-W, 2010. RNA-based antiviral immunity. Nat Rev Immunol 10, 632-44.

Dong C, Zhao J, Song L, et al., 2009. The immune responses in Chinese mitten crab Eriocheir sinensis challenged with double-stranded RNA. Fish \& Shellfish Immunology 26, 438-42.

European Food Safety Authority, 2014. International scientific workshop 'Risk assessment considerations for RNAi-based GM plants' (4-5 June 2014, Brussels, Belgium). EFSA Supporting publication 2014; 11(12):EN-705, 38 pp. doi:10.2903/sp.efsa.2014.EN-705

Flenniken ML, Andino R, 2013. Non-Specific dsRNA-Mediated Antiviral Response in the Honey Bee. PloS one 8, e77263.

Gammon DB, Mello CC, 2015. RNA interference-mediated antiviral defense in insects. Current Opinion in Insect Science 8, 111-20.

Garbian Y, Maori E, Kalev H, Shafir S, Sela I, 2012. Bidirectional Transfer of RNAi between Honey Bee and Varroa destructor: Varroa Gene Silencing Reduces Varroa Population. PLoS Pathogens 8, e1003035.

Garbutt JS, Reynolds SE, 2012. Induction of RNA interference genes by double-stranded RNA; implications for susceptibility to RNA interference. Insect Biochemistry and Molecular Biology 42, 621-8.

Grimm D, Streetz $\mathrm{KL}$, Jopling $\mathrm{CL}$, et al., 2006. Fatality in mice due to oversaturation of cellular microRNA/short hairpin RNA pathways. Nature 441, 537-41.

Hirai M, Terenius O, Li W, Faye I, 2004. Baculovirus and dsRNA induce Hemolin, but no antibacterial activity, in Antheraea pernyi. Insect Molecular Biology 13, 399-405.

Huang $H$, Qiao R, Zhao D, et al., 2009. Profiling of mismatch discrimination in RNAi enabled rational design of allele-specific siRNAs. Nucleic Acids Research 37, 7560-9.

Jackson AL, Bartz SR, Schelter J, et al., 2003. Expression profiling reveals off-target gene regulation by RNAi. Nature Biotechnology 21, 635-7.

Jackson AL, Burchard J, Schelter J, et al., 2006. Widespread siRNA "off-target" transcript silencing mediated by seed region sequence complementarity. RNA 12, 1179-87.

Jackson AL, Linsley PS, 2010. Recognizing and avoiding siRNA off-target effects for target identification and therapeutic application. Nat Rev Drug Discov 9, 57-67.

Jarosch A, Moritz RFA, 2011. Systemic RNA-interference in the honeybee Apis mellifera: Tissue dependent uptake of fluorescent siRNA after intra-abdominal application observed by laserscanning microscopy. Journal of Insect Physiology 57, 851-7.

Jarosch A, Moritz RFA, 2012. RNA interference in honeybees: off-target effects caused by dsRNA. Apidologie 43, 128-38.

Johnson MT, Gould F, 1992. Interaction of Genetically Engineered Host Plant Resistance and Natural Enemies of Heliothis virescens (Lepidoptera: Noctuidae) in Tobacco. Environmental Entomology 21, 586-97.

Kamakura M, 2011. Royalactin induces queen differentiation in honeybees. Nature, 473, 478-83.

The present document has been produced and adopted by the bodies identified above as authors. This task has been carried out exclusively by the author(s) in the context of a contract between the European Food Safety Authority and the authors, awarded following a tender procedure. The present document is published complying with the transparency principle to which the Authority is subject. It may not be considered as an output adopted by the Authority. The European Food Safety Authority reserves its rights, view and position as regards the issues addressed and the conclusions reached in the present document, without prejudice to the rights of the authors. 
Karlikow M, Goic B, Saleh M-C, 2014. RNAi and antiviral defense in Drosophila: Setting up a systemic immune response. Developmental \& Comparative Immunology 42, 85-92.

Khan AA, Betel D, Miller ML, Sander C, Leslie CS, Marks DS, 2009. Transfection of small RNAs globally perturbs gene regulation by endogenous microRNAs. Nature Biotechnology 27, 549-55.

Kingsolver MB, Huang Z, Hardy RW, 2013. Insect antiviral innate immunity: pathways, effectors, and connections. Journal of molecular biology 425, 4921-36.

Kitzmann P, Schwirz J, Schmitt-Engel C, Bucher G, 2013. RNAi phenotypes are influenced by the genetic background of the injected strain. BMC Genomics 14, 5.

Kulkarni MM, Booker M, Silver SJ, et al., 2006. Evidence of off-target effects associated with long dsRNAs in Drosophila melanogaster cell-based assays. Nat Meth 3, 833-8.

Labreuche $Y$, Veloso A, De La Vega E, et al., 2010. Non-specific activation of antiviral immunity and induction of RNA interference may engage the same pathway in the Pacific white leg shrimp Litopenaeus vannamei. Developmental \& Comparative Immunology 34, 1209-18.

Liu J, Smagghe G, Swevers L, 2013. Transcriptional response of BmToll9-1 and RNAi machinery genes to exogenous dsRNA in the midgut of Bombyx mori. Journal of Insect Physiology 59, 646-54.

Lövei GL, Andow DA, Arpaia S, 2009. Transgenic Insecticidal Crops and Natural Enemies: A Detailed Review of Laboratory Studies. Environmental Entomology 38, 293-306.

Lövei GL, Arpaia S, 2005. The impact of transgenic plants on natural enemies: a critical review of laboratory studies. Entomologia Experimentalis et Applicata 114, 1-14.

Lozano J, Gomez-Orte E, Lee H-J, Belles X, 2012. Super-induction of Dicer-2 expression by alien double-stranded RNAs: an evolutionary ancient response to viral infection? Development Genes and Evolution 222, 229-35.

Lundgren J, Jurat-Fuentes J, 2012. Chapter 13. Physiology and Ecology of Host Defense Against Microbial Invaders.

Lundgren JG, Duan JJ, 2013. RNAi-Based Insecticidal Crops: Potential Effects on Nontarget Species. Bioscience 63, 657-65.

Ma Y, Creanga A, Lum L, Beachy PA, 2006. Prevalence of off-target effects in Drosophila RNA interference screens. Nature 443, 359-63.

Mckey D, 1974. Adaptive Patterns in Alkaloid Physiology. The American Naturalist 108, 305-20.

Mutti NS, Park Y, Reese JC, Reeck GR, 2006. RNAi Knockdown of a Salivary Transcript Leading to Lethality in the Pea Aphid, Acyrthosiphon pisum. Journal of Insect Science 6, 38.

Naranjo SE, 2009. Impacts of Bt Crops on Non-Target Invertebrates and Insecticide Use Patterns. CAB Reviews: Perspectives in Agriculture, Veterinary Science, Nutrition and Natural Resources, 4, $1-11$.

Niu J, Meeus I, Smagghe G, 2016. Differential expression pattern of Vago in bumblebee (Bombus terrestris), induced by virulent and avirulent virus infections. 6, 34200.

Nunes FMF, Aleixo AC, Barchuk AR, Bomtorin AD, Grozinger CM, Simões ZLP, 2013. Non-target Effects of Green Fluorescent Protein (GFP)-derived Double-Stranded RNA (dsRNA-GFP) Used in Honey Bee RNA Interference (RNAi) Assays. Insects 4, 90-103.

Pan H, Xu L, Noland JE, Li H, Siegfried BD, Zhou X, 2016. Assessment of Potential Risks of Dietary RNAi to a Soil Micro-arthropod, Sinella curviseta Brook (Collembola: Entomobryidae). Frontiers in Plant Science 7.

The present document has been produced and adopted by the bodies identified above as authors. This task has been carried out exclusively by the author(s) in the context of a contract between the European Food Safety Authority and the authors, awarded following a tender procedure. The present document is published complying with the transparency principle to which the Authority is subject. It may not be considered as an output adopted by the Authority. The European Food Safety Authority reserves its rights, view and position as regards the issues addressed and the conclusions reached in the present document, without prejudice to the rights of the authors. 
Pan H, Yang X, Bidne K, Hellmich RL, Siegfried BD, Zhou X, 2017. Dietary Risk Assessment of vATPase A dsRNAs on Monarch Butterfly Larvae. Frontiers in Plant Science 8, 242.

Paradkar PN, Trinidad L, Voysey R, Duchemin J-B, Walker PJ, 2012. Secreted Vago restricts West Nile virus infection in Culex mosquito cells by activating the Jak-STAT pathway. Proceedings of the National Academy of Sciences of the United States of America 109, 18915-20.

Petrick JS, Brower-Toland B, Jackson AL, Kier LD, 2013. Safety assessment of food and feed from biotechnology-derived crops employing RNA-mediated gene regulation to achieve desired traits: A scientific review. Regulatory Toxicology and Pharmacology 66, 167-76.

Piot N, Snoeck S, Vanlede M, Smagghe G, Meeus I, 2015. The Effect of Oral Administration of dsRNA on Viral Replication and Mortality in Bombus terrestris. Viruses 7, 3172-85.

Price PW, Bouton CE, Gross P, Mcpheron BA, Thompson JN, Weis AE, 1980. Interactions Among Three Trophic Levels: Influence of Plants on Interactions Between Insect Herbivores and Natural Enemies. Annual Review of Ecology and Systematics 11, 41-65.

Qiu S, Adema CM, Lane T, 2005. A computational study of off-target effects of RNA interference. Nucleic Acids Research 33, 1834-47.

Robalino J, Bartlett T, Shepard E, et al., 2005. Double-Stranded RNA Induces Sequence-Specific Antiviral Silencing in Addition to Nonspecific Immunity in a Marine Shrimp: Convergence of RNA Interference and Innate Immunity in the Invertebrate Antiviral Response? Journal of Virology 79, 13561-71.

Robalino J, Bartlett TC, Chapman RW, Gross PS, Browdy CL, Warr GW, 2007. Double-stranded RNA and antiviral immunity in marine shrimp: Inducible host mechanisms and evidence for the evolution of viral counter-responses. Developmental \& Comparative Immunology 31, 539-47.

Robalino J, Browdy CL, Prior S, et al., 2004. Induction of Antiviral Immunity by Double-Stranded RNA in a Marine Invertebrate. Journal of Virology 78, 10442-8.

Robbins M, Judge A, Maclachlan I, 2009. SiRNA and innate immunity.

Runo S, Alakonya A, Machuka J, Sinha N, 2011. RNA interference as a resistance mechanism against crop parasites in Africa: a 'Trojan horse' approach. Pest Management Science 67, 129-36.

Schwarz DS, Ding $H$, Kennington $L$, et al., 2006. Designing siRNA that distinguish between genes that differ by a single nucleotide. PLoS genetics 2, 1307-18.

Sim S, Ramirez JL, Dimopoulos G, 2012. Dengue Virus Infection of the Aedes aegypti Salivary Gland and Chemosensory Apparatus Induces Genes that Modulate Infection and Blood-Feeding Behavior. PLoS Pathogens 8, e1002631.

Su J, Zhu Z, Wang Y, Zou J, Wang N, Jang S, 2009. Grass carp reovirus activates RNAi pathway in rare minnow, Gobiocypris rarus. Aquaculture 289, 1-5.

Sugahara R, Tanaka S, Jouraku A, Shiotsuki T, 2017. Geographic variation in RNAi sensitivity in the migratory locust. Gene 605, 5-11.

Swevers L, Smagghe G, 2012. Use of RNAi for Control of Insect Crop Pests. In: Smagghe G, Diaz I, eds. Arthropod-Plant Interactions: Novel Insights and Approaches for IPM. Dordrecht: Springer Netherlands, 177-97.

Tang B, Wang S, Zhang F, 2010. Two storage hexamerins from the beet armyworm Spodoptera exigua: Cloning, characterization and the effect of gene silencing on survival. BMC Molecular Biology 11, 65-.

The present document has been produced and adopted by the bodies identified above as authors. This task has been carried out exclusively by the author(s) in the context of a contract between the European Food Safety Authority and the authors, awarded following a tender procedure. The present document is published complying with the transparency principle to which the Authority is subject. It may not be considered as an output adopted by the Authority. The European Food Safety Authority reserves its rights, view and position as regards the issues addressed and the conclusions reached in the present document, without prejudice to the rights of the authors. 
Terenius O, Papanicolaou A, Garbutt JS, et al., 2011. RNA interference in Lepidoptera: An overview of successful and unsuccessful studies and implications for experimental design. Journal of Insect Physiology 57, 231-45.

United States Environmental Protection Agency 2014. SAP Minutes No. 2014-02. A Set of Scientific Issues Being Considered by the Environmental Protection Agency Regarding: RNAi Technology: Program Formulation for Human Health and Ecological Risk Assessment. http://wssa.net/wpcontent/uploads/RNAi-report_EPA-May-2014.pdf

Vélez AM, Jurzenski J, Matz N, et al., 2016. Developing an in vivo toxicity assay for RNAi risk assessment in honey bees, Apis mellifera L. Chemosphere 144, 1083-90.

Whyard S, Singh AD, Wong S, 2009. Ingested double-stranded RNAs can act as species-specific insecticides. Insect Biochemistry and Molecular Biology 39, 824-32.

Zhang H, Li H, Guan R, Miao X, 2015. Lepidopteran insect species-specific, broad-spectrum, and systemic RNA interference by spraying dsRNA on larvae. Entomologia Experimentalis et Applicata $155,218-28$.

Zheng Z-M, Tang S, Tao M, 2005. Development of resistance to RNAi in mammalian cells. Annals of the New York Academy of Sciences 1058, 105-18.

Zhou X, Xu F, Mao H, et al., 2014. Nuclear RNAi Contributes to the Silencing of Off-Target Genes and Repetitive Sequences in Caenorhabditis elegans. Genetics 197, 121-32.

Zhu J-Q, Liu S, Ma Y, et al., 2012. Improvement of Pest Resistance in Transgenic Tobacco Plants Expressing dsRNA of an Insect-Associated Gene EcR. PloS one 7, e38572.

The present document has been produced and adopted by the bodies identified above as authors. This task has been carried out exclusively by the author(s) in the context of a contract between the European Food Safety Authority and the authors, awarded following a tender procedure. The present document is published complying with the transparency principle to which the Authority is subject. It may not be considered as an output adopted by the Authority. The European Food Safety Authority reserves its rights, view and position as regards the issues addressed and the conclusions reached in the present document, without prejudice to the rights of the authors. 


\subsection{Overview of invertebrate species for which a sequenced genome is available (Task 6)}

In this Section, we present an overview of the available genomic data for invertebrates belonging to the phyla of nematodes, arthropods, molluscs and annelids.

\subsubsection{Introduction to genome sequencing in relation to RNAi}

RNAi gene silencing is based on sequence homology between the effector molecule (dsRNA, siRNA, miRNA) and the target mRNA. Therefore, the availability of sequence data can be of great importance. It can be of importance for researchers and crop protectors when designing the sRNA and for avoiding off-target effects or gene silencing effects on NTOs. Furthermore, the availability of genomic sequence data on species in a given agroecosystem might also be of interest for risk assessors in determining potential effects on NTOs in that ecosystem. However, there has been some debate on the potential use of bioinformatics to predict silencing effects in target and NTOs. One of the main issues is that for invertebrates, there is no real consensus yet on the 'rules' for siRNA/RISC binding to the homologous mRNA (see Section 5.4).

Additionally, one must also consider that most genome sequences do not have a $100 \%$ coverage of the genome, nor are they always a $100 \%$ accurate. For example sequencing mistakes may occur or inclusion of contaminant sequences (e.g., bacterial symbionts) that sometimes remain in the genome after assembly and deposition in the public database can also cause errors (Lam et al., 2012; Merchant et al., 2014). Furthermore, automatic gene-predicting algorithms are also prone to mistakes, such as the failure to detect ORFs or the failure to correctly identify exon/intron structures. An additional issue is the fact that for most species, only one genome from one strain is publicly available, the so-called reference genome. Mutations in the genomes of other strains would be unknown.

Nevertheless, having an overview of the collection of genomic data can be of use. While using bioinformatics might not fully exclude potential off-target effects, it is helpful in identifying high-risk sRNAs for some NTOs, for example when multiple fully matching $21 \mathrm{nt}$ siRNA sequence matches are present between the SRNA and a gene in the genome. Finally, the phenomenon of transitive RNAi, which entails the production of secondary siRNAs which can match regions of the target gene upstream or downstream the dsRNA triggering gene silencing, must be considered as well. This mechanism, which has been identified in the nematode C. elegans, has been discussed earlier in Section 5.1.4. So far, this mechanism has not been identified in arthropods, molluscs or annelids.

\subsubsection{Genomic data}

There are several databases which are receiving information from on-going international projects sequencing the genomes of a range of animals, microbes and plants. We present an overview of the available genomic data for invertebrates belonging to the phyla of nematodes, arthropods, molluscs and annelids extracted from four major databases or platforms:

- NCBI (Genbank): https://www.ncbi.nlm.nih.gov/

- JGI Genomes Online Databases (GOLD): https://gold.jgi.doe.gov/

- National human genome research institute (NIH): https://www.genome.gov/

- I5K (5,000 insect/arthropod genomes initiative): http://i5k.github.io/

Our search was performed on 17 February 2017, and it resulted in a list of 494 finished or ongoing invertebrate genome sequencing projects in total; 330 in arthropod species, 113 in nematodes, 40 in molluscs and 11 in annelids. Of these 494 genome sequencing projects, 333 (237 arthropods, 76 nematodes, 15 molluscs and 5 annelids) had been completed and have their genomic data publicly available on Genbank. For the other 161 genomes, which were only identified in the JGI database and

The present document has been produced and adopted by the bodies identified above as authors. This task has been carried out exclusively by the author(s) in the context of a contract between the European Food Safety Authority and the authors, awarded following a tender procedure. The present document is published complying with the transparency principle to which the Authority is subject. It may not be considered as an output adopted by the Authority. The European Food Safety Authority reserves its rights, view and position as regards the issues addressed and the conclusions reached in the present document, without prejudice to the rights of the authors. 
not in Genbank, projects are labelled 'incomplete' and are most likely still ongoing; for example, with assembly or annotation processes. Furthermore, an additional four genome sequencing projects, three Caenorhabditis strains and the chilopod Strigamia maritime were found on the NIH platform only. The I5K platform did not reveal any additional genomes that were not already found in Genbank.

The complete list of genome projects for which the data were available on 17 February 2017 is given in Table 18. The table also includes the genome Genbank ID and information on the assembly level for NCBI datasets (Contig, Scaffold or Chromosome). In genome sequencing, a contig refers to a collection of overlapping sequenced fragments. In other words, it forms a continuous stretch of sequence data created by read overlaps. These contigs can then be assembled into larger scaffolds, which still contain gaps of known length between the contigs. Several strategies are available to fill these gaps afterwards. Assembly at contig level means that nothing in the genome has been assembled beyond the level of sequenced contigs. Assembly at scaffold level means that at least some contigs have been assembled into larger scaffolds. At this level, scaffolds are still unplaced and unlocalized. Assembly at chromosome level refers to an available complete sequence for one or more chromosomes where scaffolds have been localized and placed. However, there may still be unlocalized and unplaced scaffolds. These levels give an indication of the coverage and assembly of the genome and the ability to annotate most genes in the genome. For some species there are genome projects and datasets for multiple strains. In those cases, only the so-called representative genome was referred to in the table.

Table 18: Overview of invertebrate species for which the genome sequence data was publicly available as of 17 February 2017

\begin{tabular}{llcl}
\hline Phylum & Organism & Genbank ID & Assembly level \\
\hline Arthropoda & Achipteria coleoptrata & 37199 & Scaffold \\
& Acromyrmex echinatior & 34551 & Scaffold \\
& Acyrthosiphon pisum & 29162 & Scaffold \\
& Aedes aegypti & 307340 & Scaffold \\
& Aedes albopictus & 304150 & Scaffold \\
& Agrilus planipennis & 202629 & Scaffold \\
& Amyelois transitella & 38909 & Scaffold \\
& Anopheles albimanus & 11556 & Scaffold \\
& Anopheles arabiensis & 11544 & Scaffold \\
& Anopheles atroparvus & 11555 & Scaffold \\
& Anopheles christyi & 11547 & Scaffold \\
& Anopheles culicifacies & 11552 & Scaffold \\
& Anopheles darlingi & 2624 & Contig \\
& Anopheles dirus & 11554 & Scaffold \\
Anopheles epiroticus & 11546 & Scaffold \\
& Anopheles farauti & 11553 & Contig \\
Anopheles funestus & 2697 & Scaffold \\
Anopheles gambiae & 46 & Scaffold \\
Anopheles koliensis & 36653 & Contig \\
Anopheles maculatus & 11550 & Scaffold \\
Anopheles melas & 11549 & Scaffold
\end{tabular}

The present document has been produced and adopted by the bodies identified above as authors. This task has been carried out exclusively by the author(s) in the context of a contract between the European Food Safety Authority and the authors, awarded following a tender procedure. The present document is published complying with the transparency principle to which the Authority is subject. It may not be considered as an output adopted by the Authority. The European Food Safety Authority reserves its rights, view and position as regards the issues addressed and the conclusions reached in the present document, without prejudice to the rights of the authors. 


\begin{tabular}{|c|c|c|}
\hline Anopheles merus & 11548 & Scaffold \\
\hline Anopheles minimus & 11551 & Scaffold \\
\hline Anopheles nili & 17525 & Scaffold \\
\hline Anopheles punctulatus & 36652 & Contig \\
\hline Anopheles quadriannulatus & 11545 & Scaffold \\
\hline Anopheles sinensis & 12170 & Scaffold \\
\hline Anopheles stephensi & 2653 & Scaffold \\
\hline Anoplophora glabripennis & 14033 & Scaffold \\
\hline Apis cerana & 12051 & Scaffold \\
\hline Apis dorsata & 15019 & Scaffold \\
\hline Apis florea & 2740 & Scaffold \\
\hline Apis mellifera & 937 & Chromosome \\
\hline Athalia rosae & 14027 & Scaffold \\
\hline Atta cephalotes & 2844 & Scaffold \\
\hline Atta colombica & 44113 & Scaffold \\
\hline Bactrocera cucurbitae & 11807 & Scaffold \\
\hline Bactrocera dorsalis & 10754 & Scaffold \\
\hline Bactrocera oleae & 17298 & Scaffold \\
\hline Bactrocera tryoni & 15403 & Scaffold \\
\hline Belgica antarctica & 14659 & Scaffold \\
\hline Blattella germanica & 13223 & Scaffold \\
\hline Bombus impatiens & 3415 & Scaffold \\
\hline Bombus terrestris & 2739 & Chromosome \\
\hline Bombyx mori & 76 & Scaffold \\
\hline Caligus rogercresseyi & 38161 & Contig \\
\hline Calliphora vicina & 15677 & Scaffold \\
\hline Calycopis cecrops & 44594 & Scaffold \\
\hline Camponotus floridanus & 2966 & Scaffold \\
\hline Catajapyx aquilonaris & 17772 & Scaffold \\
\hline Centruroides sculpturatus & 14105 & Scaffold \\
\hline Cephus cinctus & 14134 & Scaffold \\
\hline Cerapachys biroi & 55526 & Scaffold \\
\hline Ceratina calcarata & 45025 & Scaffold \\
\hline Ceratitis capitata & 2738 & Scaffold \\
\hline Ceratosolen solmsi & 23331 & Scaffold \\
\hline Chaoborus trivitattus & 38285 & Scaffold \\
\hline Chilo suppressalis & 13084 & Contig \\
\hline Chironomus riparius & 2978 & Scaffold \\
\hline Chironomus tentans & 35154 & Scaffold \\
\hline Cimex lectularius & 11279 & Scaffold \\
\hline Clogmia albipunctata & 38294 & Scaffold \\
\hline
\end{tabular}

The present document has been produced and adopted by the bodies identified above as authors. This task has been carried out exclusively by the author(s) in the context of a contract between the European Food Safety Authority and the authors, awarded following a tender procedure. The present document is published complying with the transparency principle to which the Authority is subject. It may not be considered as an output adopted by the Authority. The European Food Safety Authority reserves its rights, view and position as regards the issues addressed and the conclusions reached in the present document, without prejudice to the rights of the authors. 


\begin{tabular}{|c|c|c|}
\hline Coboldia fuscipes & 38275 & Scaffold \\
\hline Condylostylus patibulatus & 38287 & Scaffold \\
\hline Copidosoma floridanum & 12734 & Scaffold \\
\hline Cotesia vestalis & 12200 & Contig \\
\hline Culex quinquefasciatus & 393 & Scaffold \\
\hline Cyphomyrmex costatus & 44114 & Scaffold \\
\hline Dactylopius coccus & 36092 & Contig \\
\hline Danaus plexippus & 11702 & Scaffold \\
\hline Daphnia magna & 10953 & Scaffold \\
\hline Daphnia pulex & 288 & Scaffold \\
\hline Dendroctonus ponderosae & 11242 & Scaffold \\
\hline Dermatophagoides farinae & 9138 & Scaffold \\
\hline Diachasma alloeum & 40839 & Scaffold \\
\hline Diaphorina citri & 867 & Scaffold \\
\hline Dinoponera quadriceps & 40599 & Scaffold \\
\hline Diuraphis noxia & 24062 & Scaffold \\
\hline Drosophila albomicans & 2712 & Scaffold \\
\hline Drosophila americana & 10917 & Scaffold \\
\hline Drosophila ananassae & 244 & Scaffold \\
\hline Drosophila arizonae & 45090 & Scaffold \\
\hline Drosophila biarmipes & 3499 & Scaffold \\
\hline Drosophila bipectinata & 3489 & Scaffold \\
\hline Drosophila busckii & 38276 & Chromosome \\
\hline Drosophila elegans & 3490 & Scaffold \\
\hline Drosophila erecta & 250 & Scaffold \\
\hline Drosophila eugracilis & 6863 & Scaffold \\
\hline Drosophila ficusphila & 3491 & Scaffold \\
\hline Drosophila grimshawi & 257 & Scaffold \\
\hline Drosophila kikkawai & 3492 & Scaffold \\
\hline Drosophila melanogaster & 47 & Chromosome \\
\hline Drosophila miranda & 10915 & Scaffold \\
\hline Drosophila mojavensis & 259 & Scaffold \\
\hline Drosophila navojoa & 45091 & Scaffold \\
\hline Drosophila persimilis & 265 & Scaffold \\
\hline Drosophila pseudoobscura & 219 & Scaffold \\
\hline Drosophila rhopaloa & 6853 & Scaffold \\
\hline Drosophila sechellia & 271 & Scaffold \\
\hline Drosophila simulans & 200 & Chromosome \\
\hline Drosophila suzukii & 18317 & Scaffold \\
\hline Drosophila takahashii & 3493 & Scaffold \\
\hline Drosophila virilis & 300047 & Scaffold \\
\hline
\end{tabular}

The present document has been produced and adopted by the bodies identified above as authors. This task has been carried out exclusively by the author(s) in the context of a contract between the European Food Safety Authority and the authors, awarded following a tender procedure. The present document is published complying with the transparency principle to which the Authority is subject. It may not be considered as an output adopted by the Authority. The European Food Safety Authority reserves its rights, view and position as regards the issues addressed and the conclusions reached in the present document, without prejudice to the rights of the authors. 


\begin{tabular}{|c|c|c|}
\hline Drosophila willistoni & 251 & Scaffold \\
\hline Drosophila yakuba & 148 & Chromosome \\
\hline Dufourea novaeangliae & 39971 & Scaffold \\
\hline Ephemera danica & 14601 & Scaffold \\
\hline Ephydra gracilis & 38282 & Scaffold \\
\hline Ephydra hians & 38282 & Scaffold \\
\hline Eristalis dimidiata & 38298 & Scaffold \\
\hline Eufriesea mexicana & 44840 & Scaffold \\
\hline Eurytemora affinis & 17731 & Scaffold \\
\hline Eutreta diana & 38297 & Scaffold \\
\hline Ferrisia virgata & 44261 & Scaffold \\
\hline Fopius arisanus & 35518 & Scaffold \\
\hline Frankliniella occidentalis & 14741 & Scaffold \\
\hline Gerris buenoi & 17730 & Scaffold \\
\hline Glossina austeni & 16689 & Scaffold \\
\hline Glossina brevipalpis & 15576 & Scaffold \\
\hline Glossina fuscipes & 15034 & Scaffold \\
\hline Glossina morsitans & 55 & Scaffold \\
\hline Glossina pallidipes & 15956 & Scaffold \\
\hline Glossina palpalis & 15033 & Scaffold \\
\hline Habropoda laboriosa & 39899 & Scaffold \\
\hline Halyomorpha halys & 14101 & Scaffold \\
\hline Harpegnathos saltator & 2965 & Scaffold \\
\hline Heliconius cydno & 10843 & Scaffold \\
\hline Heliconius elevatus & 42294 & Scaffold \\
\hline Heliconius ethilla & 42293 & Scaffold \\
\hline Heliconius hecale & 33769 & Scaffold \\
\hline Heliconius ismenius & 36108 & Scaffold \\
\hline Heliconius melpomene & 10844 & Scaffold \\
\hline Heliconius numata & 12823 & Scaffold \\
\hline Heliconius pardalinus & 42296 & Scaffold \\
\hline Heliconius timareta & 15536 & Scaffold \\
\hline Hermetia illucens & 38288 & Scaffold \\
\hline Holcocephala fusca & 38300 & Scaffold \\
\hline Homalodisca vitripennis & 13454 & Scaffold \\
\hline Hyalella azteca & 16496 & Scaffold \\
\hline Hypochthonius rufulus & 37200 & Scaffold \\
\hline Hypothenemus hampei & 38265 & Scaffold \\
\hline Ixodes ricinus & 16267 & Scaffold \\
\hline Ixodes scapularis & 523 & Scaffold \\
\hline Ladona fulva & 17240 & Scaffold \\
\hline
\end{tabular}

The present document has been produced and adopted by the bodies identified above as authors. This task has been carried out exclusively by the author(s) in the context of a contract between the European Food Safety Authority and the authors, awarded following a tender procedure. The present document is published complying with the transparency principle to which the Authority is subject. It may not be considered as an output adopted by the Authority. The European Food Safety Authority reserves its rights, view and position as regards the issues addressed and the conclusions reached in the present document, without prejudice to the rights of the authors. 


\begin{tabular}{|c|c|c|}
\hline Lasioglossum albipes & 15038 & Scaffold \\
\hline Lasius niger & 14569 & Scaffold \\
\hline Latrodectus hesperus & 14107 & Scaffold \\
\hline Lepeophtheirus salmonis & 2713 & Contig \\
\hline Leptinotarsa decemlineata & 12832 & Scaffold \\
\hline Lerema accius & 40057 & Scaffold \\
\hline Limnephilus lunatus & 17773 & Scaffold \\
\hline Limulus polyphemus & 787 & Scaffold \\
\hline Linepithema humile & 2733 & Scaffold \\
\hline Liriomyza trifolii & 16235 & Scaffold \\
\hline Locusta migratoria & 10772 & Contig \\
\hline Loxosceles reclusa & 14028 & Scaffold \\
\hline Lucilia cuprina & 12732 & Scaffold \\
\hline Lucilia sericata & 8289 & Scaffold \\
\hline Lutzomiya longipalpis & 30816 & Scaffold \\
\hline Maconellicoccus hirsutus & 44264 & Scaffold \\
\hline Manduca sexta & 12037 & Scaffold \\
\hline Mayetiola destructor & 2619 & Scaffold \\
\hline Megachile rotundata & 6563 & Scaffold \\
\hline Megaselia abdita & 38299 & Scaffold \\
\hline Megaselia scalaris & 15767 & Scaffold \\
\hline Melipona quadrifasciata & 12726 & Scaffold \\
\hline Melitaea cinxia & 814 & Scaffold \\
\hline Mengenilla moldrzyki & 14989 & Contig \\
\hline Mesobuthus martensii & 14571 & Contig \\
\hline Metaseiulus occidentalis & 3487 & Scaffold \\
\hline Microplitis demolitor & 12766 & Scaffold \\
\hline Mochlonyx cinctipes & 38286 & Scaffold \\
\hline Monomorium pharaonis & 37124 & Scaffold \\
\hline Musca domestica & 14461 & Scaffold \\
\hline Nasonia giraulti & 759 & Scaffold \\
\hline Nasonia longicornis & 760 & Scaffold \\
\hline Nasonia vitripennis & 449 & Chromosome \\
\hline Nasutitermes exitiosus & 40812 & Contig \\
\hline Neobellieria bullata & 13341 & Scaffold \\
\hline Neodiprion lecontei & 39861 & Scaffold \\
\hline Nicrophorus vespilloides & 40824 & Scaffold \\
\hline Nilaparvata lugens & 2941 & Scaffold \\
\hline Oncopeltus fasciatus & 11434 & Scaffold \\
\hline Onthophagus taurus & 12827 & Scaffold \\
\hline Operophtera brumata & 39883 & Scaffold \\
\hline
\end{tabular}

The present document has been produced and adopted by the bodies identified above as authors. This task has been carried out exclusively by the author(s) in the context of a contract between the European Food Safety Authority and the authors, awarded following a tender procedure. The present document is published complying with the transparency principle to which the Authority is subject. It may not be considered as an output adopted by the Authority. The European Food Safety Authority reserves its rights, view and position as regards the issues addressed and the conclusions reached in the present document, without prejudice to the rights of the authors. 


\begin{tabular}{|c|c|c|}
\hline Orussus abietinus & 14602 & Scaffold \\
\hline Oryctes borbonicus & 41561 & Contig \\
\hline Pachypsylla venusta & 14032 & Scaffold \\
\hline Papilio glaucus & 36240 & Scaffold \\
\hline Papilio machaon & 14159 & Scaffold \\
\hline Papilio polytes & 33756 & Scaffold \\
\hline Papilio xuthus & 13942 & Scaffold \\
\hline Paracoccus marginatus & 44262 & Scaffold \\
\hline Parasteatoda tepidariorum & 13270 & Scaffold \\
\hline Parhyale hawaiensis & 15533 & Scaffold \\
\hline Pediculus humanus & 522 & Scaffold \\
\hline Phlebotomus papatasi & 10999 & Scaffold \\
\hline Phoebis sennae & 44038 & Scaffold \\
\hline Phortica variegata & 38277 & Scaffold \\
\hline Piezodorus guildinii & 34914 & Scaffold \\
\hline Platynothrus peltifer & 37201 & Scaffold \\
\hline Plutella xylostella & 11570 & Scaffold \\
\hline Pogonomyrmex barbatus & 2732 & Scaffold \\
\hline Polistes canadensis & 16494 & Scaffold \\
\hline Polistes dominula & 42066 & Scaffold \\
\hline Priacma serrata & 11831 & Contig \\
\hline Pseudococcus longispinus & 44263 & Scaffold \\
\hline Rhipicephalus microplus & 2797 & Scaffold \\
\hline Rhodnius prolixus & 447 & Scaffold \\
\hline Sarcoptes scabiei & 36095 & Scaffold \\
\hline Scaptodrosophila lebanonensis & 38279 & Scaffold \\
\hline Solenopsis invicta & 2938 & Scaffold \\
\hline Sphyracephala brevicornis & 38301 & Scaffold \\
\hline Spodoptera frugiperda & 10985 & Scaffold \\
\hline Steganacarus magnus & 8750 & Scaffold \\
\hline Stegodyphus mimosarum & 12925 & Scaffold \\
\hline Stomoxys calcitrans & 11278 & Scaffold \\
\hline Strigamia maritima & 790 & Scaffold \\
\hline Teleopsis dalmanni & 13232 & Scaffold \\
\hline Tephritis californica & 38315 & Scaffold \\
\hline Tetranychus urticae & 2710 & Scaffold \\
\hline Themira minor & 38280 & Scaffold \\
\hline Tipula oleracea & 38316 & Scaffold \\
\hline Trachymyrmex cornetzi & 44115 & Scaffold \\
\hline Trachymyrmex septentrionalis & 44116 & Scaffold \\
\hline Trachymyrmex zeteki & 44394 & Scaffold \\
\hline
\end{tabular}

The present document has been produced and adopted by the bodies identified above as authors. This task has been carried out exclusively by the author(s) in the context of a contract between the European Food Safety Authority and the authors, awarded following a tender procedure. The present document is published complying with the transparency principle to which the Authority is subject. It may not be considered as an output adopted by the Authority. The European Food Safety Authority reserves its rights, view and position as regards the issues addressed and the conclusions reached in the present document, without prejudice to the rights of the authors. 


\begin{tabular}{|c|c|c|c|}
\hline & Tribolium castaneum & 216 & Chromosome \\
\hline & Trichogramma pretiosum & 14106 & Scaffold \\
\hline & Trionymus perrisii & 44268 & Scaffold \\
\hline & Triops cancriformis & 7259 & contig \\
\hline & Trupanea jonesi & 38283 & Scaffold \\
\hline & unclassified Sarcophagidae & 40370 & Scaffold \\
\hline & Unclassified Trichoceridae & 38278 & Scaffold \\
\hline & Varroa destructor & 937 & Contig \\
\hline & Vollenhovia emeryi & 36511 & Scaffold \\
\hline & Wasmannia auropunctata & 36651 & Scaffold \\
\hline & Zootermopsis nevadensis & 17755 & Scaffold \\
\hline \multirow[t]{30}{*}{ Nematoda } & Ancylostoma ceylanicum & 10936 & Scaffold \\
\hline & Ancylostoma duodenale & 348 & Scaffold \\
\hline & Angiostrongylus cantonensis & 30176 & Scaffold \\
\hline & Angiostrongylus costaricensis & 9101 & Scaffold \\
\hline & Anisakis simplex & 7896 & Scaffold \\
\hline & Ascaris lumbricoides & 11969 & Scaffold \\
\hline & Ascaris suum & 350 & Scaffold \\
\hline & Brugia malayi & 42 & Scaffold \\
\hline & Brugia pahangi & 13249 & Scaffold \\
\hline & Brugia timori & 36524 & Scaffold \\
\hline & Bursaphelenchus xylophilus & 11822 & Contig \\
\hline & Caenorhabditis angaria & 3127 & Scaffold \\
\hline & Caenorhabditis brenneri & 254 & Scaffold \\
\hline & Caenorhabditis briggsae & 40 & Chromosome \\
\hline & Caenorhabditis elegans & 41 & Chromosome \\
\hline & Caenorhabditis japonica & 252 & Scaffold \\
\hline & Caenorhabditis nigoni & 44874 & Scaffold \\
\hline & Caenorhabditis remanei & 253 & scaffold \\
\hline & Caenorhabditis tropicalis & 35261 & Scaffold \\
\hline & Cylicostephanus goldi & 36526 & Scaffold \\
\hline & Dictyocaulus viviparus & 779 & Scaffold \\
\hline & Dirofilaria immitis & 10757 & Contig \\
\hline & Ditylenchus destructor & 43946 & Scaffold \\
\hline & Dracunculus medinensis & 10838 & Scaffold \\
\hline & Elaeophora elaphi & 24454 & Scaffold \\
\hline & Enterobius vermicularis & 8694 & Scaffold \\
\hline & Globodera pallida & 32442 & Scaffold \\
\hline & Globodera rostochiensis & 44826 & Scaffold \\
\hline & Gongylonema pulchrum & 36527 & Scaffold \\
\hline & Haemonchus contortus & 16936 & Scaffold \\
\hline
\end{tabular}

The present document has been produced and adopted by the bodies identified above as authors. This task has been carried out exclusively by the author(s) in the context of a contract between the European Food Safety Authority and the authors, awarded following a tender procedure. The present document is published complying with the transparency principle to which the Authority is subject. It may not be considered as an output adopted by the Authority. The European Food Safety Authority reserves its rights, view and position as regards the issues addressed and the conclusions reached in the present document, without prejudice to the rights of the authors. 


\begin{tabular}{|c|c|c|}
\hline Haemonchus placei & 36529 & Scaffold \\
\hline Heligmosomoides polygyrus & 36519 & Scaffold \\
\hline Heterodera glycines & 852 & Scaffold \\
\hline Heterorhabditis bacteriophora & 481 & Scaffold \\
\hline Loa loa & 2686 & Contig \\
\hline Meloidogyne floridensis & 33826 & Scaffold \\
\hline Meloidogyne hapla & 260 & Contig \\
\hline Meloidogyne incognita & 281 & Contig \\
\hline Necator americanus & 770 & Scaffold \\
\hline Nippostrongylus brasiliensis & 11002 & Scaffold \\
\hline Oesophagostomum dentatum & 782 & Scaffold \\
\hline Onchocerca flexuosa & 11948 & Scaffold \\
\hline Onchocerca ochengi & 18268 & Scaffold \\
\hline Onchocerca volvulus & 2687 & Scaffold \\
\hline Oscheius sp. MCB & 36317 & Contig \\
\hline Panagrellus redivivus & 16242 & Scaffold \\
\hline Parascaris equorum & 36531 & Scaffold \\
\hline Parastrongyloides trichosuri & 36401 & Scaffold \\
\hline Pristionchus pacificus & 246 & Contig \\
\hline Rhabditophanes sp. & 36407 & Scaffold \\
\hline Romanomermis culicivorax & 23995 & Scaffold \\
\hline Rotylenchulus reniformis & 11277 & Scaffold \\
\hline Soboliphyme baturini & 36532 & Scaffold \\
\hline Steinernema carpocapsae & 2699 & Scaffold \\
\hline Steinernema feltiae & 17805 & Scaffold \\
\hline Steinernema glaseri & 17808 & Scaffold \\
\hline Steinernema monticolum & 17810 & Scaffold \\
\hline Steinernema scapterisci & 17807 & Scaffold \\
\hline Strongyloides papillosus & 36387 & Scaffold \\
\hline Strongyloides ratti & 3496 & Chromosome \\
\hline Strongyloides stercoralis & 13317 & Scaffold \\
\hline Strongyloides venezuelensis & 12700 & Scaffold \\
\hline Strongylus vulgaris & 9335 & Scaffold \\
\hline Subanguina moxae & 37144 & Scaffold \\
\hline Syphacia muris & 36393 & Scaffold \\
\hline Thelazia callipaeda & 14733 & Scaffold \\
\hline Toxocara canis & 8517 & Scaffold \\
\hline Trichinella & 41654 & Scaffold \\
\hline Trichinella britovi & 35242 & Scaffold \\
\hline Trichinella murrelli & 35264 & Scaffold \\
\hline Trichinella nativa & 35238 & Scaffold \\
\hline
\end{tabular}

The present document has been produced and adopted by the bodies identified above as authors. This task has been carried out exclusively by the author(s) in the context of a contract between the European Food Safety Authority and the authors, awarded following a tender procedure. The present document is published complying with the transparency principle to which the Authority is subject. It may not be considered as an output adopted by the Authority. The European Food Safety Authority reserves its rights, view and position as regards the issues addressed and the conclusions reached in the present document, without prejudice to the rights of the authors. 
Trichinella nelsoni

Trichinella papuae

35237

35252

Trichinella patagoniensis

Trichinella pseudospiralis

Trichinella spiralis

Trichinella zimbabwensis

Trichuris muris

Trichuris suis

Trichuris trichiura

Wuchereria bancrofti

Mollusca

Annelida

Aplysia californica

Bankia setacea

Biomphalaria glabrata

Conus tribblei

Corbicula fluminea

Crassostrea gigas

Crassostrea virginica

Dreissena polymorpha

Lottia gigantea

Lymnaea stagnalis

Mizuhopecten yessoensis

Mytilus galloprovincialis

Octopus bimaculoides

Pinctada martensi

Radix auricularia

Amynthas corticis

Capitella teleta

Eisenia fetida

Helobdella robusta

Hydroides elegans
41656

11844

35251

31518

13427

13417

2616

443

10465

357

40372

15808

10758

398

13187

15113

14639

12193

12190

41501

55961

55350

40146

15118

12810

15112

45775
Scaffold

Scaffold

Scaffold

Scaffold

Scaffold

Scaffold

Scaffold

Scaffold

Scaffold

Scaffold

Scaffold

Contig

Scaffold

Scaffold

Contig

Scaffold

Chromosome

Contig

Scaffold

Contig

Scaffold

Contig

Scaffold

Chromosome

Scaffold

Scaffold

Scaffold

Scaffold

Scaffold

Scaffold

\subsubsection{References}

Lam HY, Clark MJ, Chen R, et al., 2012. Performance comparison of whole-genome sequencing platforms. Nature Biotechnology, 30, 78-82.

Merchant S, Wood DE and Salzberg SL, 2014. Unexpected cross-species contamination in genome sequencing projects. Peer], 2, e675.

The present document has been produced and adopted by the bodies identified above as authors. This task has been carried out exclusively by the author(s) in the context of a contract between the European Food Safety Authority and the authors, awarded following a tender procedure. The present document is published complying with the transparency principle to which the Authority is subject. It may not be considered as an output adopted by the Authority. The European Food Safety Authority reserves its rights, view and position as regards the issues addressed and the conclusions reached in the present document, without prejudice to the rights of the authors. 


\section{Conclusions}

In this Technical Report, an overview is given on the state of the art regarding RNAi in invertebrates. First, a systematic literature search was performed to identify all peer-reviewed studies conducted on RNAi in nematodes, arthropods, molluscs and annelids, leading to the collection of 13,867 studies. After this initial search, 4,612 studies which dealt with oral exposure to small RNAs were identified, analysed and listed in Annex 2. When we examined these studies, we found that most research on RNAi was conducted in the phyla of nematodes $(27 \%)$ and arthropods $(71 \%)$. Only a very limited number of studies have investigated RNAi in molluscs and annelids. In both nematodes and arthropods, there is a clear level of variability in sensitivity to RNAi. For example, nematodes belonging to the Caenorhabditis genus appear to be more sensitive than many other nematode species, due to an expanded RNAi-related gene repertoire in the genome. Similarly, in arthropods, a high degree of variability also exists, especially in the sensitivity of species to oral RNAi. Generally Coleoptera, which is comprised of beetles and weevils, appear to be more sensitive to RNAi than species in other orders and require smaller doses to elicit successful gene silencing. This indicates that the dose of sRNA to which target and NTOs are exposed is important when designing RNAi systems in plants.

In Section 5.1 and 5.3, several factors that influence this variability have been identified, including sRNA stability in the invertebrate body, the efficiency of cellular uptake of sRNAs, the presence of siRNA amplification systems, viral infections which could interfere with the RNAi machinery and RNAirelated gene repertoire. These factors have implications regarding the potential for silencing effects in non-target species and also the development of resistance to (oral) RNAi. Furthermore, we also found that while the cellular uptake machinery in nematodes (notably $C$. elegans) has been well-studied and described, this is not the case for species belonging to arthropods, molluscs and annelids. This cellular uptake has been studied in a limited number of insect species, but it seems clear that arthropods do not possess most of the genes involved in C. elegans SRNA specific uptake. While similar systems might exist in both phyla, they have not been entirely elucidated in arthropods yet.

In Section 5.2, we consider possible exposure routes and the fate of sRNAs produced in GM plants. Several direct or indirect exposure routes were identified. While methods exist to identify the sRNA expression levels in plants, we found very few studies actually reporting these. Interestingly, dsRNA seems to be very short-lived in the environment. Laboratory studies investigating the persistence of dsRNA in soil and aquatic environments indicated that most dsRNA is degraded within 48 hours. However, no field studies investigating the fate of dsRNA derived from GM plants are available yet.

We also reported on the potential for adverse effects on NTOs caused by sRNA exposure (Section 5.4). Several mechanisms have been identified in the literature which could cause unintended effects, including sequence-dependent silencing effects caused by sequence homology between SRNA and a (homologous) gene in the genome and sequence-independent effects such as immune system stimulation and RNAi machinery saturation. Only a limited number of studies have investigated unintended silencing effects in NTOs and all of these were conducted in insects using dsRNA targeting either the DvSnf7 or the DvV-ATPase in the Western Corn Rootworm. These studies indicated that successful gene silencing requires a high degree of homology and at least a full 20nt match between the dsRNA sequence and the mRNA. However, we could not find any study really fundamentally investigating siRNA specificity in invertebrates, making it difficult to identify a general rule on the necessary homology between dsRNA and mRNA to cause gene silencing. Phylogenetic relatedness between target and non-target species might favor the possible occurrence of non-target effects, though in some cases silencing has occurred after ingestion of dsRNA in test organisms belonging to different insect orders. Immune stimulation and RNAi machinery saturation have been proposed as potential adverse effects in some commentaries, but hard evidence that this might occur, let alone be a cause for a fitness cost in NTOs, is lacking. Furthermore, the studies conducted in the context of the ERA of the dsSnf7-expressing GM maize seemed to indicate the very high specificity of this product, especially when compared to other plant produced pesticides used to date.

The present document has been produced and adopted by the bodies identified above as authors. This task has been carried out exclusively by the author(s) in the context of a contract between the European Food Safety Authority and the authors, awarded following a tender procedure. The present document is published complying with the transparency principle to which the Authority is subject. It may not be considered as an output adopted by the Authority. The European Food Safety Authority reserves its rights, view and position as regards the issues addressed and the conclusions reached in the present document, without prejudice to the rights of the authors. 
An interesting question in the context of RNAi ERA is whether bioinformatics could play a useful role. In Section 5.5, we have compiled a list of invertebrate genomes that have been sequenced and are publically available and we discuss the potential usefulness of bioinformatics in predicting adverse effects in NTOs. In total, we identified 494 species for which the genomic data is available. This number represents only a very small part of the diversity of potential non-target invertebrates which could be exposed to SRNA in agroecosystems. Furthermore, the incomplete coverage of most genomes and the uncertainty regarding the necessary homology between SRNA and mRNA to cause gene silencing means that, at this time, bioinformatics alone cannot be relied on to predict the absence of any possible silencing effects. However, bioinformatics could still be useful at an early stage to identify highly conserved potential high risk sRNA for some NTOs, for example when multiple fully matching $21 \mathrm{nt}$ siRNA sequence matches are present between the sRNA and a gene in the genome.

\section{Recommendations}

The evidence collected here indicates that RNAi is a very promising pest control method, compared to many of the currently available pest control strategies, due to the possibility of designing highly species-specific dsRNA and their short-lived persistence in the environment. However, there are several gaps in our knowledge of this mechanism, for example on the necessary homology between siRNA and mRNA and the potential adverse effects on immune systems of invertebrate NTOs. In order to support environmental risk assessments of RNAi GM plants, this report has indicated several areas which warrant further study and more bioinformatics information is required on a wider diversity of the invertebrate species occurring in agro-ecosystems. Bioinformatics could be a useful tool in ERA to screen and predict some sRNAs that have a $21 \mathrm{nt}$ match against any of the available genomes but until we have more research information we cannot use it to determine the absence of silencing effects in NTOs with any certainty.

The present document has been produced and adopted by the bodies identified above as authors. This task has been carried out exclusively by the author(s) in the context of a contract between the European Food Safety Authority and the authors, awarded following a tender procedure. The present document is published complying with the transparency principle to which the Authority is subject. It may not be considered as an output adopted by the Authority. The European Food Safety Authority reserves its rights, view and position as regards the issues addressed and the conclusions reached in the present document, without prejudice to the rights of the authors. 


\section{Abbreviations}

\begin{tabular}{|c|c|}
\hline$A B C$ & ATP-binding cassette \\
\hline Bt & Bacillus thuringhiensis \\
\hline CDS & coding sequence \\
\hline CPB & Colorado potato beetle \\
\hline diRNA & defective interfering RNA \\
\hline dsRNA & double-stranded RNA \\
\hline EFSA & European Food Safety Authority \\
\hline EPA & Environmental Protection Agency \\
\hline ERA & environmental risk assessment \\
\hline FITC & fluorescein isothiocyanate \\
\hline GFP & green fluorescent protein \\
\hline GM & genetically modified \\
\hline MEEC & maximum expected environmental concentration \\
\hline miRNA & micro RNA \\
\hline mRNA & messenger RNA \\
\hline ncRNA & non-coding RNA \\
\hline NTO & non-target organism \\
\hline qRT-PCR & quantitative reversed transcription polymerase chain reaction \\
\hline RdRP & RNA dependant RNA polymerase \\
\hline RISC & RNA-induced silencing complex \\
\hline RNAi & RNA interference \\
\hline rsd & RNAi spreading defective \\
\hline SAGO & siRNA-specific Argonaute \\
\hline SCR & Southern corn rootworm \\
\hline shRNA & short hairpin RNA \\
\hline sid & spreading RNA interference defective \\
\hline SiRNA & small interfering RNA \\
\hline sRNA & short RNA \\
\hline TGN & Trans-Golgi network \\
\hline TO & target organism \\
\hline UTR & untranslated region \\
\hline viRNA & viral RNA \\
\hline VSR & viral suppressor of RNAi \\
\hline WAGO & Worm-specific argonaute \\
\hline WCR & Western corn rootworm \\
\hline WoS & Web of Science \\
\hline
\end{tabular}

The present document has been produced and adopted by the bodies identified above as authors. This task has been carried out exclusively by the author(s) in the context of a contract between the European Food Safety Authority and the authors, awarded following a tender procedure. The present document is published complying with the transparency principle to which the Authority is subject. It may not be considered as an output adopted by the Authority. The European Food Safety Authority reserves its rights, view and position as regards the issues addressed and the conclusions reached in the present document, without prejudice to the rights of the authors. 


\section{Appendix A - List of reference studies used to assess the relevance of the results from the defined search string in Web of Science}

\begin{tabular}{|c|c|c|c|c|}
\hline No. & Authors & Year & $\begin{array}{l}\text { Journal, volume, } \\
\text { pages }\end{array}$ & Title \\
\hline 1 & Abdellatef et al. & 2015 & $\begin{array}{l}\text { Plant Biotechnology } \\
\text { Journal } 13,849-857\end{array}$ & $\begin{array}{l}\text { Silencing the expression of the salivary sheath } \\
\text { protein causes transgenerational feeding } \\
\text { suppression in the aphid Sitobion avenae }\end{array}$ \\
\hline 2 & $\begin{array}{l}\text { Abdel-Latief and } \\
\text { Hoffmann }\end{array}$ & 2014 & Peptides 53, 172-184 & $\begin{array}{l}\text { Functional activity of allatotropin and } \\
\text { allatostatin in the pupal stage of a } \\
\text { holometablous insect, Tribolium castaneum } \\
\text { (Coleoptera, Tenebrionidae) }\end{array}$ \\
\hline 3 & Aditi et al. & 2015 & $\begin{array}{l}\text { Transgenic Research 24, } \\
791-801\end{array}$ & $\begin{array}{l}\text { Transgenic plants over-expressing insect- } \\
\text { specific microRNA acquire insecticidal activity } \\
\text { against Helicoverpa armigera: an alternative to } \\
\text { Bt-toxin technology }\end{array}$ \\
\hline 4 & Ahmad et al. & 2016 & $\begin{array}{l}\text { Transgenic Research } 25, \\
1-17\end{array}$ & $\begin{array}{l}\text { Transportable data from non-target arthropod } \\
\text { field studies for the environmental risk } \\
\text { assessment of genetically modified maize } \\
\text { expressing an insecticidal double-stranded } \\
\text { RNA }\end{array}$ \\
\hline 5 & Allen and Walker & 2012 & $\begin{array}{l}\text { Journal of Insect } \\
\text { Physiology 58, 391-396 }\end{array}$ & $\begin{array}{l}\text { Saliva of Lygus lineolaris digests double } \\
\text { stranded ribonucleic acids }\end{array}$ \\
\hline 6 & Araujo et al & 2006 & $\begin{array}{l}\text { Insect Biochemistry and } \\
\text { Molecular Biology 36, } \\
683-693\end{array}$ & $\begin{array}{l}\text { RNA interference of the salivary gland } \\
\text { nitrophorin } 2 \text { in the triatomine bug Rhodnius } \\
\text { prolixus (Hemiptera: Reduviidae) by dsRNA } \\
\text { ingestion or injection }\end{array}$ \\
\hline 7 & Aronstein et al & 2006 & $\begin{array}{l}\text { Journal of Apicultural } \\
\text { Research } 45,20-24\end{array}$ & $\begin{array}{l}\text { SID-1 is implicated in systemic gene silencing } \\
\text { in the honey bee }\end{array}$ \\
\hline 8 & Bachman et al & 2013 & $\begin{array}{l}\text { Transgenic Research 22, } \\
1207-1222\end{array}$ & $\begin{array}{l}\text { Characterization of the spectrum of insecticidal } \\
\text { activity of a double-stranded RNA with } \\
\text { targeted activity against western } \\
\text { cornrootworm (Diabrotica virgifera virgifera Le } \\
\text { Conte) }\end{array}$ \\
\hline 9 & Baum, et al & 2007 & $\begin{array}{l}\text { Nature Biotechnology 25, } \\
1322-1326\end{array}$ & $\begin{array}{l}\text { Control of coleopteran insect pests through } \\
\text { RNA interference }\end{array}$ \\
\hline 10 & Bautista et al & 2009 & $\begin{array}{l}\text { Insect Biochemistry and } \\
\text { Molecular Biology 39, 38- } \\
46\end{array}$ & $\begin{array}{l}\text { RNA interference-mediated knockdown of a } \\
\text { cytochrome P450, CYP6BG1, from the } \\
\text { diamondback moth, Plutella xylostella, reduces } \\
\text { larval resistance to permethrin }\end{array}$ \\
\hline 11 & Belles & 2010 & $\begin{array}{l}\text { Annual Review of } \\
\text { Entomology 55, 111-128 }\end{array}$ & $\begin{array}{l}\text { Beyond Drosophila: RNAi in vivo and functional } \\
\text { genomics in insects }\end{array}$ \\
\hline 12 & Blandin et al & 2002 & $\begin{array}{l}\text { EMBO Reports 3, 852- } \\
856\end{array}$ & $\begin{array}{l}\text { Reverse genetics in the mosquito Anopheles } \\
\text { gambiae: targeted disruption of the Defensin } \\
\text { gene }\end{array}$ \\
\hline 13 & Boisson et al & 2006 & $\begin{array}{l}\text { FEBS Letters 580, 1988- } \\
1992\end{array}$ & $\begin{array}{l}\text { Gene silencing in mosquito salivary glands by } \\
\text { RNAi }\end{array}$ \\
\hline 14 & Bolognesi et al & 2012 & PLoS ONE 7, e47534 & $\begin{array}{l}\text { Characterizing the mechanism of action of } \\
\text { double-stranded RNA activity against western } \\
\text { corn rootworm (Diabrotica virgifera virgifera } \\
\text { LeConte) }\end{array}$ \\
\hline 15 & $\begin{array}{l}\text { Burand and } \\
\text { Hunter }\end{array}$ & 2013 & $\begin{array}{l}\text { Journal of Invertebrate } \\
\text { Pathology } 112, \text { S68-S74 }\end{array}$ & RNAi: future in insect management \\
\hline 16 & Chen et al & 2013 & Insect Biochemistry and & Conserved microRNAs miR-8-5p and miR-2a- \\
\hline
\end{tabular}

The present document has been produced and adopted by the bodies identified above as authors. This task has been carried out exclusively by the author(s) in the context of a contract between the European Food Safety Authority and the authors, awarded following a tender procedure. The present document is published complying with the transparency principle to which the Authority is subject. It may not be considered as an output adopted by the Authority. The European Food Safety Authority reserves its rights, view and position as regards the issues addressed and the conclusions reached in the present document, without prejudice to the rights of the authors. 
Molecular Biology 43, 839-848

17 Chen et al

2014 PLoS Pathogens 10, e1004261

18

Christiaens et al

2014

Peptides 53, 307-314

19

Chu et al

2014

Pesticide Biochemistry and Physiology 110, 1-6

20 Clemens et al

2000

Proceedings of the National Academy of Sciences of the United States of America 97, 6499-6503

21 Coleman et al

2014 Journal of Experimental Botany 66, 541-548

22 Coy et al

Journal of Applied

Entomology 136, 741-748

23

Drake et al

2012

Journal of Visualized

Experiments, e3479

24

Dubelman et al

2014

PLOS ONE 9, e93155

25

Dutta et al

2014

Frontiers in Microbiology

5, nr. 760

26

El-Shesheny et

2013

PLOS ONE 8, e65392 al

27

Fabioux et al

2009

FEBS Journal 276, 2566-

2573

28 Fang et al

2011

PLoS ONE 6, e21860

29

Feinberg and

Hunter

30 Fire et al

2003

Science 301, 1545-1547

1998

Nature $391,806-811$

31

Garbian et al

2012

PLoS Pathogens 8, e1003035

32

Garbutt and Reynolds

33

Ghanim et al

34

Gong et al

35
2012

Insect Biochemistry and Molecular Biology 42, 621-628

2007 Insect Biochemistry and Molecular Biology 37, 732-738

2013 PLOS ONE 8, e62990

2004 Development Growth \& Differentiation 46, 83-95
$3 p$ modulate chitin biosynthesis in response to 20- hydroxyecdysone signaling in the brown planthopper, Nilaparvata lugens

Israeli acute paralysis virus: epidemiology, pathogenesis and implications for honey bee health

DsRNA degradation in the pea aphid

(Acyrthosiphon pisum) associated with lack of response in RNAi feeding and injection assay Differential effects of RNAi treatments on field populations of the western corn rootworm Use of double-stranded RNA interference in Drosophila cell lines to dissect signal transduction pathways

Persistence and transgenerational effect of plant-mediated RNAi in aphids

Gene silencing in adult Aedes aegypti

mosquitoes through oral delivery of doublestranded RNA

RNAi-mediated gene knockdown and in vivo diuresis assay in adult female Aedes aegypti mosquitoes

Environmental fate of double-stranded RNA in agricultural soils

The status of RNAi-based transgenic research in plant nematology

Silencing abnormal wing disc gene of the Asian citrus psyllid, Diaphorina citri disrupts adult wing development and increases nymph mortality

In vivo RNA interference in oyster-vasa silencing inhibits germ cell development Identification of genes directly involved in shell formation and their functions in pearl oyster;

Pinctada fucata

Transport of dsRNA into cells by the transmembrane protein SID-1

Potent and specific genetic interference by double-stranded RNA in Caenorhabditis elegans

Bidirectional transfer of RNAi between honey bee and Varroa destructor: Varroa gene silencing reduces Varroa population Induction of RNA interference genes by double-stranded RNA; implications for susceptibility to RNA interference Tissue-specific gene silencing by RNA interference in the whitefly Bemisia tabaci

Testing insecticidal activity of novel chemically synthesized siRNA against Plutella xylostella under laboratory and field conditions Muscle differentiation in tentacles of Sepia officinalis Mollusc is regulated by muscle regulatory factors MRF related proteins

The present document has been produced and adopted by the bodies identified above as authors. This task has been carried out exclusively by the author(s) in the context of a contract between the European Food Safety Authority and the authors, awarded following a tender procedure. The present document is published complying with the transparency principle to which the Authority is subject. It may not be considered as an output adopted by the Authority. The European Food Safety Authority reserves its rights, view and position as regards the issues addressed and the conclusions reached in the present document, without prejudice to the rights of the authors. 
2013

Crop Protection 45, 36-40

37

Guo et al

2014

PLoS ONE 9, e97410

38

Hajeri et al

2014 Journal of Biotechnology $176,42-49$

39

Hiruta et al

40

Hossain et al

2008

BMC Biot

Insect Biochemistry and Molecular Biology 38, 1001-1007

41 Huang et al

2006 Proceedings of the National Academy of Sciences of the United States of America 103, 14302-14306

42 Huvenne and

2010 Journal of Insect

Smagghe

43 Ivashuta et al

44 Jaubert-

Possamai et al

45

Jiang et al

46

Jiang et al

47

Kamath and

Ahringer

48

Kamath et al

49

Kamath et al

Karim et al

51

Kato et al

52

Kennerdell and Carthew

53

Khajuria, et al

2015

Insect Biochemistry and Molecular Biology 63, 5462

$54 \quad$ Killiny et al

2014

PLoS ONE 9, e110536 63
Recent advances in RNA interference research in insects: implications for future insect pest management strategies

Plant-Generated Artificial Small RNAs Mediated Aphid Resistance

Citrus tristeza virus-based RNAi in citrus plants induces gene silencing in Diaphorina citri, a phloem-sap sucking insect vector of citrus greening disease (Huanglongbing)

Development of a microinjection system for RNA interference in the water flea Daphnia pulex

Expression of 20-hydroxyecdysone- induced genes in the silkworm brain and their functional analysis in post-embryonic development

Engineering broad root-knot resistance in transgenic plants by RNAi silencing of a conserved and essential root-knot nematode parasitism gene

Mechanisms of dsRNA uptake in insects and potential of RNAi for pest control: a review Environmental RNAi in herbivorous insects

2007 BMC Biotechnology 7, nr. Gene knockdown by RNAi in the pea aphid

Acyrthosiphon pisum

In vivo and in vitro knockdown of FREP2 gene expression in the snail Biomphalaria glabrata using RNA interference

MicroRNA-281 regulates the expression of ecdysone receptor (EcR) isoform $B$ in the silkworm, Bombyx mori

Genome-wide RNAi screening in

Caenorhabditis elegans

Effectiveness of specific RNA-mediated

interference through ingested double stranded

RNA in Caenorhabditis elegans

Systematic functional analysis of the

Caenorhabditis elegans genome using RNAi

Functional genomics tool: gene silencing in

Ixodes scapularis eggs and nymphs by

electroporated dsRNA

Development of an RNA interference method in the cladoceran crustacean Daphnia magna

Use of dsRNA-mediated genetic interference to demonstrate that frizzled and frizzled 2 act in the wingless pathway

Parental RNA interference of genes involved in embryonic development of the western corn rootworm, Diabrotica virgifera virgifera LeConte

Double-stranded RNA uptake through topical application, mediates silencing of five CYP4 genes and suppresses insecticide resistance in Diaphorina citri

Suppression of nitric oxide NO-dependent behaviour by double-stranded RNA-mediated

The present document has been produced and adopted by the bodies identified above as authors. This task has been carried out exclusively by the author(s) in the context of a contract between the European Food Safety Authority and the authors, awarded following a tender procedure. The present document is published complying with the transparency principle to which the Authority is subject. It may not be considered as an output adopted by the Authority. The European Food Safety Authority reserves its rights, view and position as regards the issues addressed and the conclusions reached in the present document, without prejudice to the rights of the authors. 
2012 PLoS ONE 7, e31347

57

La Fauce and

Owens

58 La Fauce and Owens

59 Labreuche et al

2010 Developmental and Comparative Immunology $34,1209-1218$

60 Lee et al

2001 Learning and Memory 8, 220-226

61 Lee et al

62 Lee et al

\section{Cell 117, 69-81}

2011 Insect biochemistry and Molecular Biology 41, 236-243

PLoS ONE 10, e0118622
Li et al

Journal of Applied Entomology 139, 432-445

65

Li-Byarlay et al

2013

Proceedings of the National Academy of Sciences of the United States of America 110, 12750-12755

66 Liu et al

2010 Insect Biochemistry and Molecular Biology 40, 666-671

67 Liu et al

2013 Journal of Insect Physiology 59, 646-654

68 Luan et al

2013 Insect Biochemistry and Molecular Biology 43, 740-746

69 Luo et al

2012 RNA Biology 9, 663-671

70 Maeda et al

2001 Current Biology 11, 171176

71 Mao et al

2007 Nature Biotechnology 25, 1307-1313

72 Mao et al

2011 Transgenic Research 20, 665-673 silencing of a neuronal NO synthase gene

Tobacco rattle virus vector: a rapid and transient means of silencing Manduca sexta genes by plant mediated RNA interference Suppression of Penaeus merguiensis densovirus following oral delivery of live bacteria expressing dsRNA in the house cricket (Acheta domesticus) model

RNA interference reduces PmergDNV expression and replication in an in vivo cricket model

Non-specific activation of antiviral immunity and induction of RNA interference may engage the same pathway in the Pacific white leg shrimp Litopenaeus vannamei

Overexpression of and RNA interference with the CCAAT enhancer-binding protein on long term facilitation of Aplysia sensory to motor synapses

Distinct roles for Drosophila Dicer-1 and Dicer2 in the siRNA/miRNA silencing pathways RNA interference of pheromone biosynthesisactivating neuropeptide receptor suppresses mating behavior by inhibiting sex pheromone production in Plutella xylostella (L)

Independent action between DvSnf7 RNA and Cry3Bb1 protein in southern corn rootworm, Diabrotica undecimpunctata howardi and Colorado potato beetle, Leptinotarsa decemlineata

Long dsRNA but not siRNA initiates RNAi in western corn rootworm larvae and adults RNA interference knockdown of DNA methyltransferase 3 affects gene alternative splicing in the honey bee

Gene knockdown by intro-thoracic injection of double-stranded RNA in the brown planthopper, Nilaparvata lugens

Transcriptional response of BmToll9-1 and RNAi machinery genes to exogenous dsRNA in the midgut of Bombyx mori

Silencing the ecdysone synthesis and signaling pathway genes disrupts nymphal development in the whitefly

The SID-1 double-stranded RNA transporter is not required for systemic RNAi in the migratory locust

Large-scale analysis of gene function in

Caenorhabditis elegans by high-throughput RNAi

Silencing a cotton bollworm P450

monooxygenase gene by plant-mediated RNAi impairs larval tolerance of gossypol Cotton plants expressing CYP6AE14 doublestranded RNA show enhanced resistance to

The present document has been produced and adopted by the bodies identified above as authors. This task has been carried out exclusively by the author(s) in the context of a contract between the European Food Safety Authority and the authors, awarded following a tender procedure. The present document is published complying with the transparency principle to which the Authority is subject. It may not be considered as an output adopted by the Authority. The European Food Safety Authority reserves its rights, view and position as regards the issues addressed and the conclusions reached in the present document, without prejudice to the rights of the authors. 


\begin{tabular}{|c|c|c|c|}
\hline 73 & Mao et al & 2013 & $\begin{array}{l}\text { Plant Molecular Biology } \\
83,119-129\end{array}$ \\
\hline 74 & Mao and Zeng & 2012 & PLoS ONE 7, e48718 \\
\hline 75 & Mao and Zeng & 2014 & $\begin{array}{l}\text { Transgenic Research 23, } \\
145-152\end{array}$ \\
\hline 76 & Maori et al & 2009 & $\begin{array}{l}\text { Insect Molecular Biology } \\
18,55-60\end{array}$ \\
\hline 77 & Marques et al & 2013 & $\begin{array}{l}\text { PLoS Pathogens 9, } \\
\text { e1003579 }\end{array}$ \\
\hline 78 & Martin et al & 2006 & $\begin{array}{l}\text { Journal of Insect } \\
\text { Physiology } 52,410-416\end{array}$ \\
\hline 79 & Miller et al & 2008 & $\begin{array}{l}\text { Development Genes and } \\
\text { Evolution } 218,505-510\end{array}$ \\
\hline 80 & Miller, et al & 2012 & PLOS ONE 7, e47431 \\
\hline
\end{tabular}

$81 \quad$ Minakuchi et al 2008 FEBS Journal 275, 29192931

\begin{tabular}{|c|c|c|c|}
\hline 82 & Miyata et al & 2014 & PLoS ONE 9, e101661 \\
\hline 83 & Miyoshi et al & 2010 & $\begin{array}{l}\text { Nature Structural and } \\
\text { Molecular Biology 17, } \\
\text { 1024-1026 }\end{array}$ \\
\hline 84 & Mussig et al & 2010 & $\begin{array}{l}\text { Journal of Neuroscience } \\
30,7817-7825\end{array}$ \\
\hline 85 & Mutti et al & 2006 & $\begin{array}{l}\text { Journal of Insect Science } \\
6,1-7\end{array}$ \\
\hline 86 & Leigh et al & 2015 & $\begin{array}{l}\text { Aquaculture } 437,360- \\
365\end{array}$ \\
\hline 87 & Paldi et al & 2010 & $\begin{array}{l}\text { Applied and } \\
\text { Environmental } \\
\text { Microbiology 76, 5960- } \\
5964\end{array}$ \\
\hline 88 & Pitino et al & 2011 & PLoS ONE 6, e25709 \\
\hline 89 & Pridgeon et al & 2008 & $\begin{array}{l}\text { Journal of Medical } \\
\text { Entomology } 45,414-420\end{array}$ \\
\hline 90 & $\begin{array}{l}\text { Ramaseshadri et } \\
\text { al }\end{array}$ & 2013 & PLoS ONE 8, e54270 \\
\hline 91 & $\begin{array}{l}\text { Rangasamy and } \\
\text { Siegfried }\end{array}$ & 2012 & $\begin{array}{l}\text { Pest Management } \\
\text { Science } 68,587-591\end{array}$ \\
\hline 92 & Roberts et al & 2015 & $\begin{array}{l}\text { Frontiers in Plant Science } \\
6, \mathrm{nr} .953\end{array}$ \\
\hline
\end{tabular}

bollworms

Cysteine protease enhances plant-mediated bollworm RNA interference

Feeding-based RNA interference of a gap gene is lethal to the pea aphid, Acyrthosiphon pisum Plant-mediated RNAi of a gap gene enhanced tobacco tolerance against the Myzus persicae IAPV, a bee-affecting virus associated with colony collapse disorder can be silenced by dsRNA ingestion

Functional specialization of the small interfering RNA pathway in response to virus infection

RNAi studies reveal a conserved role for RXR in molting in the cockroach Blattella germanica Larval RNAi in Drosophila?

Dissecting systemic RNA interference in the red flour beetle Tribolium castaneum: parameters affecting the efficiency of RNAi RNAi-mediated knockdown of juvenile hormone acid O-methyltransferase gene causes precocious metamorphosis in the red flour beetle Tribolium castaneum Establishing an in vivo assay system to identify components involved in environmental RNA interference in the western corn rootworm A direct role for Hsp90 in pre-RISC formation in Drosophila

Acute disruption of the NMDA receptor subunit NR1 in the honeybee brain selectively impairs memory formation

RNAi knockdown of a salivary transcript leading to lethality in the pea aphid, Acyrthosiphon pisum

Diet-delivery of therapeutic RNA interference in live E coli against pre-existing Penaeus merguiensis hepandensovirus in Penaeus merguiensis

Effective gene silencing of a microsporidian parasite associated with honey bee (Apis mellifera) colony declines
Silencing of aphid genes by dsRNA feeding from plants
Topically applied AaeIAP1 double-stranded RNA kills female adults of Aedes aegypti Physiological and cellular responses caused by RNAi- mediated suppression of Snf7 orthologue in western corn rootworm (Diabrotica virgifera) larvae Validation of RNA interference in western corn rootworm diabrotica virgifera virgifera Le Conte (Coleoptera: Chrysomelidae) adults Biosafety research for non-target organism risk assessment of RNAi-based GE plants

The present document has been produced and adopted by the bodies identified above as authors. This task has been carried out exclusively by the author(s) in the context of a contract between the European Food Safety Authority and the authors, awarded following a tender procedure. The present document is published complying with the transparency principle to which the Authority is subject. It may not be considered as an output adopted by the Authority. The European Food Safety Authority reserves its rights, view and position as regards the issues addressed and the conclusions reached in the present document, without prejudice to the rights of the authors. 
Rosa et al

2010

Journal of RNAi and gene silencing 6, 361-366

95 Rosa et al

2012

Pest Management Science 68, 995-1002

Nature $458,346-350$

Scott, et al

2013

Seitz et al

2011

Journal of Insect Physiology 59, 1212-1221

Insect Biochemistry and Molecular Biology 39, 110

100

Shih and Hunter

2011

RNA

101

Sijen et al

2001

Cell 107, 465-476

102 Snow et al

RNA Biology 10, 11071116

103 Steeves et al

2006

Functior 33, 991-999

104

Surakasi et al

2011

Journal of Insect

Physiology 57, 1537-1544

105

Terenius et al

2011

Journal of Insect

Physiology 57, 231-245

106

Thakur et al

2014

PLOS ONE 9, e87235

107

Tian et al

2009

PLoS ONE 4, e6225

108

Timmons et al

2001

Gene 263, 103-112

109

Tomoyasu et al

2008

Genome Biology 9, R10

110 Turner et al

2006

Insect Molecular Biology 15, 383-391

111 Upadhyay et al

2011

Journal of Biosciences 36, 153-161

112 van Rij et al

2006

Genes and Development 20, 2985-2995

113 Walshe et al

2009

Insect Molecular Biology 18, 11-19

114 Wang et al
Developmental and Comparative Immunology
RNA interference to reveal roles of beta-Nacetylglucosaminidase gene during molting process in Locusta migratoria

RNAi effects on actin mRNAs in Homalodisca vitripennis cells

RNA interference is induced in the glassy winged sharpshooter Homalodisca vitripennis by actin dsRNA

Antiviral immunity in Drosophila requires systemic RNA interference spread

Towards the elements of successful insect RNAi

A 5'-uridine amplifies miRNA/miRNA* asymmetry in Drosophila by promoting RNAinduced silencing complex formation A water-specific aquaporin involved in aphid osmoregulation

SID-1 is a dsRNA-selective dsRNA-gated channel RNA

On the role of RNA amplification in dsRNAtriggered gene silencing

Ineffective delivery of diet-derived microRNAs to recipient animal organisms

Transgenic soybeans expressing siRNAs specific to a major sperm protein gene suppress Heterodera glycines reproduction RNA interference of beta1 integrin subunit impairs development and immune responses of the beet armyworm, Spodoptera exigua RNA interference in Lepidoptera: an overview of successful and unsuccessful studies and implications for experimental design Enhanced Whitefly Resistance in Transgenic Tobacco Plants Expressing Double Stranded RNA of V-ATPase A Gene

Developmental control of a lepidopteran pest Spodoptera exigua by ingestion of bacteria expressing dsRNA of a non-midgut gene Ingestion of bacterially expressed dsRNAs can produce specific and potent genetic interference in Caenorhabditis elegans Exploring systemic RNA interference in insects: a genomewide survey for RNAi genes in Tribolium

RNA interference in the light brown apple moth, Epiphyas postvittana (Walker) induced by doublestranded RNA feeding

RNA interference for the control of whiteflies (Bemisia tabaci) by oral route

The RNA silencing endonuclease Argonaute 2 mediates specific antiviral immunity in Drosophila melanogaster Prolonged gene knockdown in the tsetse fly Glossina by feeding double stranded RNA A primitive Toll-like receptor signalling pathway in mollusk Zhikong scallop Chlamys

The present document has been produced and adopted by the bodies identified above as authors. This task has been carried out exclusively by the author(s) in the context of a contract between the European Food Safety Authority and the authors, awarded following a tender procedure. The present document is published complying with the transparency principle to which the Authority is subject. It may not be considered as an output adopted by the Authority. The European Food Safety Authority reserves its rights, view and position as regards the issues addressed and the conclusions reached in the present document, without prejudice to the rights of the authors. 
115

Whitten et al

2016

116 Whyard et al

2009

Proceedings of the Royal Society B 283, 20160042

Insect Biochemistry and

Molecular Biology 39, 824-832

117 Winston et al

2002 Science 295, 2456-2459

118 Winston et al

2007 Proceedings of the National Academy of Sciences of the United States of America 104, 10565-10570

119 Wuriyanghan et

2011 PLOS ONE 6, e27736 al

120 Wuriyanghan and Falk

121 Xu et al

122 Yadav et al

123 Yigit et al

124 Zha et al

125 Zhang et al

2010 Insect Molecular Biology 19, 683-693

126 Zhang et al

2013

BMC Genomics 14, 1-15

127 Zhang et al

2015

Science 347, 991-994

128 Zhang et al

2015

Entomologia

Experimentalis Et Applicata 155, 218-228

129 Zhou et al 2008 Insect Biochemistry and Molecular Biology 38, 805-815

130 Zhu et al

2011 Pest Management Science 67, 175-182

131 Zhu et al

2012 PLOS ONE 7, e38572 farreri

Symbiont-mediated RNA interference in insects

Ingested double-stranded RNAs can act as species-specific insecticides

Systemic RNAi in C. elegans requires the putative transmembrane protein sid-1 Caenorhabditis elegans SID-2 is required for environmental RNA interference

Oral delivery of double-stranded RNAs and siRNAs induces RNAi effects in the potato/tomato psyllid, Bactericerca cockerelli RNA Interference towards the Potato Psyllid, Is Induced in Plants Infected with Recombinant Tobacco mosaic virus (TMV)

Juvenile hormone and insulin regulate trehalose homeostasis in the red flour beetle, Tribolium castaneum

Hostgenerated double stranded RNA induces RNAi in plantparasitic nematodes and protects the host from infection

Analysis of the $C$. elegans Argonaute family reveals that distinct Argonautes act sequentially during RNAi

Knockdown of midgut genes by dsRNAtransgenic plant-mediated RNA interference in the hemipteran insect Nilaparvata lugens Chitosan/double-stranded RNA nanoparticlemediated RNA interference to silence chitin synthase genes through larval feeding in the African malaria mosquito (Anopheles gambiae) Identifying potential RNAi targets in grain aphid (Sitobion avenae F.) based on transcriptome profiling of its alimentary canal after feeding on wheat plants

Full crop protection from an insect pest by expression of long double-stranded RNAs in plastids

Lepidopteran insect species-specific, broadspectrum, and systemic RNA interference by spraying dsRNA on larvae

RNA interference in the termite Reticulitermes flavipes through ingestion of double-stranded RNA

Ingested RNA interference for managing the populations of the Colorado potato beetle, Leptinotarsa decemlineata

Improvement of pest resistance in transgenic tobacco plants expressing dsRNA of an insectassociated gene EcR

The present document has been produced and adopted by the bodies identified above as authors. This task has been carried out exclusively by the author(s) in the context of a contract between the European Food Safety Authority and the authors, awarded following a tender procedure. The present document is published complying with the transparency principle to which the Authority is subject. It may not be considered as an output adopted by the Authority. The European Food Safety Authority reserves its rights, view and position as regards the issues addressed and the conclusions reached in the present document, without prejudice to the rights of the authors. 
Appendix B - List of invertebrate species or genera used in RNAi studies

\begin{tabular}{|c|c|c|c|}
\hline Phylum & Subphylum/Class & Species & No. of studies \\
\hline \multicolumn{4}{|c|}{ Single species studies } \\
\hline \multirow{3}{*}{ Annelida } & & & 7 \\
\hline & Clitellata & Hirudo medicinalis & 6 \\
\hline & & Hirudo verbana & 1 \\
\hline \multirow[t]{34}{*}{ Arthropoda } & & & 3,420 \\
\hline & Chelicerata & & 149 \\
\hline & & Achaearanea tepidariorum & 8 \\
\hline & & Amblyomma americanum & 16 \\
\hline & & Amblyomma hebraeum & 2 \\
\hline & & Amblyomma maculatum & 7 \\
\hline & & Amblyomma variegatum & 1 \\
\hline & & Aquarius paludum & 1 \\
\hline & & Boophilus microplus & 1 \\
\hline & & Cupiennius salei & 6 \\
\hline & & Dermacentor marginatus & 1 \\
\hline & & Dermacentor variabilis & 7 \\
\hline & & Dermanyssus gallinae & 1 \\
\hline & & Dermatophagoides pteronyssinus & 1 \\
\hline & & Haemaphysalis longicornis & 29 \\
\hline & & Hyalomma anatolicum & 1 \\
\hline & & Ixodes ricinus & 12 \\
\hline & & Ixodes scapularis & 20 \\
\hline & & Limnoporus dissortis & 2 \\
\hline & & Metaseiulus occidentalis & 5 \\
\hline & & Ornithodoros erraticus & 1 \\
\hline & & Ornithodoros moubata & 3 \\
\hline & & Panonychus citri & 1 \\
\hline & & Parasteatoda tepidariorum & 1 \\
\hline & & Phytoseiulus persimilis & 1 \\
\hline & & Rhipicephalus decoloratus & 1 \\
\hline & & Rhipicephalus evertsi evertsi & 1 \\
\hline & & Rhipicephalus haemaphysaloides & 4 \\
\hline & & Rhipicephalus microplus & 5 \\
\hline & & Rhipicephalus sanguineus & 3 \\
\hline & & Tetranychus cinnabarinus & 1 \\
\hline & & Tetranychus urticae & 3 \\
\hline & & Varroa destructor & 3 \\
\hline & Collembola & & 1 \\
\hline
\end{tabular}

The present document has been produced and adopted by the bodies identified above as authors. This task has been carried out exclusively by the author(s) in the context of a contract between the European Food Safety Authority and the authors, awarded following a tender procedure. The present document is published complying with the transparency principle to which the Authority is subject. It may not be considered as an output adopted by the Authority. The European Food Safety Authority reserves its rights, view and position as regards the issues addressed and the conclusions reached in the present document, without prejudice to the rights of the authors. 
Orchesella cincta $\quad 1$

Crustacea

Amphibalanus amphitrite 1

Artemia diapause 1

Artemia franciscana $\quad 5$

Artemia parthenogenetica $\quad 7$

Artemia salina 2

Artemia sp 1

Caligus rogercresseyi 1

Callinectes sapidus 1

Cherax quadricarinatus $\quad 2$

Daphnia magna 2

Daphnia melanica 1

Daphnia pulex 1

Daphnia pulicaria 1

Eriocheir sinensis $\quad 7$

Exopalaemon carinicauda 1

Fenneropenaeus chinensis $\quad 14$

Fenneropenaeus merguiensis 1

Lepeophtheirus salmonis $\quad 6$

Litopenaeus schmitti 1

Litopenaeus stylirostris 1

Litopenaeus vannamei $\quad 88$

Macrobrachium nipponense $\quad 6$

Macrobrachium rosenbergii $\quad 8$

Mallotus japonicus $\quad 2$

Marsupenaeus japonicus $\quad 39$

Metapenaeus ensis 3

Pacifastacus leniusculus $\quad 5$

Penaeus chinensis 1

Penaeus japonicus 2

Penaeus merguiensis $\quad 1$

Penaeus monodon $\quad 71$

Penaeus vannamei 4

Portunus trituberculatus 1

Procambarus clarkii 9

Scylla paramamosain 1

Tigriopus californicus 1

Uca pugilator

$\begin{array}{ll}\text { Insecta } & 2,970\end{array}$

Achaea janata $\quad 1$

Acheta domesticus $\quad 6$

The present document has been produced and adopted by the bodies identified above as authors. This task has been carried out exclusively by the author(s) in the context of a contract between the European Food Safety Authority and the authors, awarded following a tender procedure. The present document is published complying with the transparency principle to which the Authority is subject. It may not be considered as an output adopted by the Authority. The European Food Safety Authority reserves its rights, view and position as regards the issues addressed and the conclusions reached in the present document, without prejudice to the rights of the authors. 
Acyrthosiphon pisum $\quad 15$

Aedes aegypti 133

Aedes albopictus 16

Aedes fluviatilis 1

Aedes taeniorhynchus 1

Agrilus planipennis 1

Agrotis ipsilon 1

Allonemobius socius $\quad 1$

Alphitobius diaperinus 1

Anastrepha obliqua 1

Anastrepha suspensa 1

Annigeres subalbatus $\quad 1$

Anopheles aquasalis $\quad 2$

Anopheles coluzzii 2

Anopheles dirus 3

Anopheles gambiae $\quad 66$

Anopheles quadrimaculatus 1

Anopheles stephensi $\quad 4$

Antheraea assama $\quad 1$

Antheraea pernyi 7

Anthonomus grandis 1

Aperiona germari 1

Aphis gossypii $\quad 7$

Apis cerana 4

Apis mellifera $\quad 78$

Apis sp 1

Apolygus lucorum 2

Apteronemobius asahinai 3

Araneae spp 1

Armigeres subalbatus 10

Arthropoda spp 1

Astylus atromaculatus 1

Athalia rosae 1

Autographa californica $\quad 4$

Bactericera cockerelli 5

Bactrocera dorsalis $\quad 23$

Bactrocera minax 1

Bactrocera oleae 1

Bemisia tabaci 15

Bicyclus anynana 1

Blattella germanica $\quad 39$

Bombus ignitus 3

The present document has been produced and adopted by the bodies identified above as authors. This task has been carried out exclusively by the author(s) in the context of a contract between the European Food Safety Authority and the authors, awarded following a tender procedure. The present document is published complying with the transparency principle to which the Authority is subject. It may not be considered as an output adopted by the Authority. The European Food Safety Authority reserves its rights, view and position as regards the issues addressed and the conclusions reached in the present document, without prejudice to the rights of the authors. 
Bombus terrestris 3

Bombyx mandarina 1

Bombyx mori 176

Camponotus floridanus 2

Carabidae spp. 1

Ceratitis capitata 7

Chaetocnema pulicaria 1

Chilo infuscatellus $\quad 1$

Chilo suppressalis $\quad 4$

Choristoneura fumiferana 2

Chrysomela populi 2

Chrysopa perla 1

Chrysopa septempunctata 1

Chrysoperla spp. 1

Chymomyza costata $\quad 1$

Cimex lectularius 4

Circulifer haematoceps 1

Coccinellidae spp. 1

Cochliomyia hominivorax 1

Cochliomyia macellaria $\quad 1$

Colaphellus bowringi 1

Coleomegilla maculata 1

Coptotermes formosanus 1

Corcyra cephalonica 1

Cosmopolites sordidus 1

Cotesia plutellae 1

Cryptotermes secundus 2

Culex pipiens 19

Culex quinquefasciatus 9

Culicoides sonorensis 1

Cyclommatus metallifer 2

Cydia pomonella 1

Cylas puncticollis $\quad 1$

Dalbulus maidis 1

Danaus plexippus 1

Delphacidae spp. 1

Dendroctonus armandi 1

Dendroctonus ponderosae 1

Dermestes maculatus 1

Diabrotica undecimpunctata howardi 3

Diabrotica virgifera virgifera $\quad 17$

Diacamma sp 1

The present document has been produced and adopted by the bodies identified above as authors. This task has been carried out exclusively by the author(s) in the context of a contract between the European Food Safety Authority and the authors, awarded following a tender procedure. The present document is published complying with the transparency principle to which the Authority is subject. It may not be considered as an output adopted by the Authority. The European Food Safety Authority reserves its rights, view and position as regards the issues addressed and the conclusions reached in the present document, without prejudice to the rights of the authors. 
Diachasmimorpha longicaudata 1

Diaphorina citri 4

Diatraea saccharalis $\quad 2$

Diploptera punctata 5

Diuraphis noxia 1

Dolichopus spp 1

Drosophila brahma

Drosophila melanogaster $\quad 1,243$

Drosophila pseudoobscura 1

Drosophila suzukii 2

Epicauta chinensis $\quad 1$

Epilachna varivestis 1

Epiphyas postvittana 1

Episyrphus balteatus 2

Eurygaster integriceps 1

Euschistus heros 1

Euxesta stigmatias 1

Eyprepocnemis plorans 2

Forficulidae spp 1

Frankliniella occidentalis 2

Gastrophysa atrocyanea 3

Geocoris spp 1

Georgecraigius atropalpus 1

Gerris buenoi 1

Glossina morsitans morsitans $\quad 16$

Glossina palpalis palpalis $\quad 1$

Graminella nigrifrons 1

Grapholita molesta $\quad 1$

Gryllus bimaculatus 31

Gryllus caudal 1

Halyomorpha halys 2

Harmonia axyridis 3

Helicoverpa armigera $\quad 67$

Helicoverpa assulta $\quad 1$

Helicoverpa zea 3

Heliothis virescens $\quad 2$

Henosepilachna vigintioctopunctata $\quad 1$

Hippodamia convergens 1

Hodotermopsis sjostedti 1

Homalodisca vitripennis 3

Homoptera spp 1

Hyalophora cecropia $\quad 2$

The present document has been produced and adopted by the bodies identified above as authors. This task has been carried out exclusively by the author(s) in the context of a contract between the European Food Safety Authority and the authors, awarded following a tender procedure. The present document is published complying with the transparency principle to which the Authority is subject. It may not be considered as an output adopted by the Authority. The European Food Safety Authority reserves its rights, view and position as regards the issues addressed and the conclusions reached in the present document, without prejudice to the rights of the authors. 
Hymenoptera spp 1

Hyphantria cunea $\quad 1$

Laodelphax striatellus $\quad 24$

Leptinotarsa decemlineata $\quad 32$

Leptopilina boulardi 1

Locusta migratoria $\quad 39$

Lucilia cuprina 1

Lucilia sericata 3

Lutzomyia longipalpis $\quad 4$

Lygus lineolaris 3

Lymantria dispar $\quad 5$

Maecolapsis sp 1

Mamestra brassicae $\quad 2$

Mamestra configurata

Manduca sexta $\quad 27$

Mayetiola destructor 3

Megacopta punctatissima 1

Megaselia abdita

Microplitis demolitor $\quad 1$

Microplitis mediator $\quad 1$

Modicogryllus siamensis 1

Monochamus alternatus 2

Musca domestica 6

Mythimna separata $\quad 2$

Myzus persicae $\quad 11$

Nasonia vitripennis 13

Nasutitermes takasagoensis $\quad 1$

Nephotettix cincticeps 5

Nilaparvata lugens $\quad 55$

Nitidulidae spp 1

Notoxus monodon $\quad 1$

Nysius plebeius $\quad 2$

Oncopeltus fasciatus $\quad 22$

Onthophagus binodis 3

Onthophagus nigriventris 1

Onthophagus sagittarius 3

Onthophagus sp 2

Onthophagus taurus $\quad 2$

Orius insidiosus 2

Ostrinia furnacalis $\quad 8$

Ostrinia nubilalis 3

Papilio xuthus $\quad 2$

The present document has been produced and adopted by the bodies identified above as authors. This task has been carried out exclusively by the author(s) in the context of a contract between the European Food Safety Authority and the authors, awarded following a tender procedure. The present document is published complying with the transparency principle to which the Authority is subject. It may not be considered as an output adopted by the Authority. The European Food Safety Authority reserves its rights, view and position as regards the issues addressed and the conclusions reached in the present document, without prejudice to the rights of the authors. 
Pardosa pseudoannulata 1

Pectinophora gossypiella $\quad 1$

Pediobius foveolatus 1

Peregrinus maidis 1

Periplaneta americana $\quad 8$

Phaedon cochleariae $\quad 5$

Phalacrus politus 1

Phenacoccus solenopsis 2

Phlebotomus papatasi 1

Phyllotreta striolata 3

Pieris rapae 4

Planococcus citri 2

Plautia stali $\quad 1$

Plodia interpunctella

Plutella xylostella $\quad 42$

Poecilus chalcites $\quad 1$

Polistes metricus 1

Protaetia brevitarsis 1

Pseudaletia separata 1

Pseudoplusia includens 1

Pteromalus puparum 1

Pyrrhocoris apterus 3

Recilia dorsalis 3

Reticulitermes flavipes $\quad 6$

Reticulitermes speratus $\quad 2$

Rhodnius prolixus $\quad 25$

Rhopalosiphum pad 1

Rhynchosciara americana 1

Riptortus pedestris $\quad 5$

Romalea microptera $\quad 1$

Sarcophaga crassipalpis 2

Sarcophaga peregrina $\quad 2$

Schistocerca americana $\quad 2$

Schistocerca gregaria $\quad 21$

Schizaphis graminum 1

Scirpophaga incertulas $\quad 1$

Sesamia nonagrioides 1

Sitobion avenae 9

Sitophilus zeamais $\quad 2$

Sogatella furcifera $\quad 8$

Solenopsis invicta $\quad 4$

Spodoptera exigua $\quad 36$

The present document has been produced and adopted by the bodies identified above as authors. This task has been carried out exclusively by the author(s) in the context of a contract between the European Food Safety Authority and the authors, awarded following a tender procedure. The present document is published complying with the transparency principle to which the Authority is subject. It may not be considered as an output adopted by the Authority. The European Food Safety Authority reserves its rights, view and position as regards the issues addressed and the conclusions reached in the present document, without prejudice to the rights of the authors. 


\begin{tabular}{|c|c|c|}
\hline & Spodoptera frugiperda & 30 \\
\hline & Spodoptera littoralis & 8 \\
\hline & Spodoptera litura & 25 \\
\hline & Tenebrio molitor & 10 \\
\hline & Thermobia domestica & 6 \\
\hline & Toxomerus spp & 1 \\
\hline & Toxoptera citricida & 1 \\
\hline & Triatoma brasiliensis & 2 \\
\hline & Triatoma infestans & 1 \\
\hline & Tribolium castaneum & 184 \\
\hline & Tribolium orthodenticle & 1 \\
\hline & Tribolium zerknullt & 1 \\
\hline & Trichoplusia ni & 5 \\
\hline & Trilocha varians & 1 \\
\hline & Trypoxylus dichotomus & 1 \\
\hline & Tuta absoluta & 1 \\
\hline & Xanthine dehydrogenase & 1 \\
\hline & Zootermopsis nevadensis & 2 \\
\hline \multirow[t]{19}{*}{ Mollusca } & & 67 \\
\hline & Aplysia californica & 3 \\
\hline & Aplysia sp & 3 \\
\hline & Aplysia synapsin & 1 \\
\hline & Argopecten purpuratus & 1 \\
\hline & Biomphalaria glabrata & 7 \\
\hline & Chlamys farreri & 9 \\
\hline & Crassostrea angulata & 1 \\
\hline & Crassostrea gigas & 10 \\
\hline & Cyclina sinensis & 2 \\
\hline & Haliotis diversicolor & 1 \\
\hline & Hyriopsis cumingii & 1 \\
\hline & Lymnaea stagnalis & 10 \\
\hline & Meretrix meretrix & 2 \\
\hline & Nipponacmea fuscoviridis & 1 \\
\hline & Pinctada fucata & 10 \\
\hline & Pinctada martensii & 3 \\
\hline & Saccostrea glomerata & 1 \\
\hline & Sepia officinali & 1 \\
\hline \multirow[t]{5}{*}{ Nematoda } & & 1292 \\
\hline & Acrobeloides sp & 1 \\
\hline & Aphelenchoides besseyi & 2 \\
\hline & Aphelenchus avenae & 1 \\
\hline & Ascaris suum & 2 \\
\hline
\end{tabular}

The present document has been produced and adopted by the bodies identified above as authors. This task has been carried out exclusively by the author(s) in the context of a contract between the European Food Safety Authority and the authors, awarded following a tender procedure. The present document is published complying with the transparency principle to which the Authority is subject. It may not be considered as an output adopted by the Authority. The European Food Safety Authority reserves its rights, view and position as regards the issues addressed and the conclusions reached in the present document, without prejudice to the rights of the authors. 
Bursaphelenchus xylophilus $\quad 15$

Caenorhabditis briggsae $\quad 14$

Caenorhabditis elegans 1109

Caenorhabditis nigoni 1

Caenorhabditis remanei 1

Ditylenchus destructor 2

Globodera pallida 6

Globodera rostochiensis 4

Haemonchus contortus $\quad 6$

Heligmosomoides polygyrus 1

Heterodera avenae $\quad 5$

Heterodera glycines 16

Heterodera schachtii $\quad 8$

Heterorhabditis bacteriophora 2

Meloidogyn paranaensis 2

Meloidogyne arenaria 3

Meloidogyne artiellia $\quad 1$

Meloidogyne chitwoodi

Meloidogyne graminicola 2

Meloidogyne hapla 3

Meloidogyne incognita $\quad 44$

Meloidogyne javanica $\quad 9$

Mesorhabditis sp 1

Nippostrongylus brasiliensis $\quad 1$

Oesophagostomum dentatum 1

Onchocerca volvulus 1

Oscheius $s p$

Panagrellus redivivus 1

Panagrolaimus superbus $\quad 1$

Pratylenchus coffeae $\quad 1$

Pratylenchus penetrans 1

Pratylenchus thornei 1

Pratylenchus vulnus 2

Pratylenchus zeae $\quad 1$

Pristionchus pacificus $\quad 2$

$\begin{array}{ll}\text { Radopholus similis } & 7\end{array}$

Rhabditis sp 1

Teladorsagia circumcincta 1

Trichinella spiralis $\quad 2$

Trichostrongylus colubriformis $\quad 1$

Environments - Soil 1

The present document has been produced and adopted by the bodies identified above as authors. This task has been carried out exclusively by the author(s) in the context of a contract between the European Food Safety Authority and the authors, awarded following a tender procedure. The present document is published complying with the transparency principle to which the Authority is subject. It may not be considered as an output adopted by the Authority. The European Food Safety Authority reserves its rights, view and position as regards the issues addressed and the conclusions reached in the present document, without prejudice to the rights of the authors. 


\section{Appendix C - List of publications considered relevant for Task 3 "Environmental exposure and fate of dsRNA SIRNA and miRNA" - Plant expression and intergenerational transmission}

\begin{tabular}{|c|c|c|c|c|}
\hline No. & Authors & Year & Journal, volume, pages & Title \\
\hline 1 & Abdellatef et al. & 2015 & $\begin{array}{l}\text { Plant Biotechnology Journal 13, 849- } \\
57\end{array}$ & $\begin{array}{l}\text { Silencing the expression of the salivary sheath protein causes transgenerational feeding } \\
\text { suppression in the aphid Sitobion avenae }\end{array}$ \\
\hline 2 & Agrawal et al. & 2015 & Transgenic Research 24, 791-801 & $\begin{array}{l}\text { Transgenic plants over-expressing insect-specific microRNA acquire insecticidal activity } \\
\text { against Helicoverpa armigera: an alternative to Bt-toxin technology }\end{array}$ \\
\hline 3 & Alcazar et al. & 2008 & Genetics $180,1275-1288$ & $\begin{array}{l}\text { Transmission dynamics of heritable silencing induced by double-stranded RNA in } \\
\text { Caenorhabditis elegans }\end{array}$ \\
\hline 4 & $\begin{array}{l}\text { J. Antonino de Souza } \\
\text { Junior et al. }\end{array}$ & 2013 & PLoS ONE 8, e85364 & $\begin{array}{l}\text { Knocking-down Meloidogyne incognita proteases by plant-delivered dsRNA has negative } \\
\text { pleiotropic effect on nematode vigor }\end{array}$ \\
\hline 5 & Apone et al. & 2014 & Journal of Insect Science 14 & $\begin{array}{l}\text { Targeting the diuretic hormone receptor to control the cotton leafworm, Spodoptera } \\
\text { littoralis }\end{array}$ \\
\hline 6 & Baum et al. & 2007 & Nat Biotech 25, 1322-1326 & Control of coleopteran insect pests through RNA interference \\
\hline 7 & Bhatia et al. & 2012 & PLoS ONE 7, e46343 & Host generated siRNAs attenuate expression of serine protease gene in Myzus persicae \\
\hline 8 & Calderon-Urrea et al. & 2012 & $\begin{array}{l}\text { Plant Biotechnology Reports 6, 263- } \\
274\end{array}$ & $\begin{array}{l}\text { Overexpression of sense and antisense ced-9 in tobacco plants confers resistance to } \\
\text { Meloidogyne incognita }\end{array}$ \\
\hline 9 & Charlton et al. & 2010 & $\begin{array}{l}\text { International Journal for Parasitology } \\
40,855-864\end{array}$ & $\begin{array}{l}\text { Additive effects of plant expressed double-stranded RNAs on root-knot nematode } \\
\text { development }\end{array}$ \\
\hline 10 & Chen et al. & 2006 & $\begin{array}{l}\text { Journal of Integrative Plant Biology } \\
48,1458-1465\end{array}$ & $\begin{array}{l}\text { Functional characterization of } \mathrm{Mi} \text {, a root-knot nematode resistance gene from tomato } \\
\text { (Lycopersicon esculentum L.) }\end{array}$ \\
\hline 11 & Chi et al. & 2016 & $\begin{array}{l}\text { International Journal for Parasitology } \\
46,105-113\end{array}$ & $\begin{array}{l}\text { Exposure to double-stranded RNA mediated by tobacco rattle virus leads to } \\
\text { transcription up-regulation of effector gene Mi-vap-2 from Meloidogyne incognita and } \\
\text { promotion of pathogenicity in progeny }\end{array}$ \\
\hline 12 & Chronis et al. & 2013 & The Plant Journal 74, 185-196 & $\begin{array}{l}\text { A ubiquitin carboxyl extension protein secreted from a plant-parasitic nematode } \\
\text { Globodera rostochiensis is cleaved in planta to promote plant parasitism }\end{array}$ \\
\hline 13 & Yang et al. & 2013 & PLoS ONE 8, e69463 & $\begin{array}{l}\text { Molecular characteristics and efficacy of } 16 \mathrm{D} 10 \text { siRNAs in inhibiting root-knot nematode } \\
\text { infection in transgenic grape hairy roots }\end{array}$ \\
\hline \multicolumn{4}{|c|}{ www.efsa.europa.eu/publications } & EFSA Supporting publication 2018:EN-142 \\
\hline
\end{tabular}


14 Clement et al.

2009 The Plant Cell 21, 2963-2979

15 Pitino et al.

16 Coleman et al.

2011 PLoS ONE 6, e25709

2015 Journal of Experimental Botany 66, 541-548

17 Collins et al.

2010 Journal of Biotechnology 150, S116S116

18 Dinh et al.

2014 Phytopathology 104, 1098-1106

19 Dinh et al.

2014 Nematology 16, 669-682

20 Dinh et al.

2015 Journal of Nematology 47, 71-78

21 Dutta et al.

2015 Frontiers in Microbiology 6, 260

22 Elzinga et al.

2014 Molecular Plant-Microbe Interactions 27, 747-756

23 Fairbairn et al.

2007 Planta 226, 1525-1533

24 Guo et al.

25 Fuchs et al.

2014 PLoS ONE 9, e97410

2007 Journal of Plant Pathology 89, 5-12

26 Hajeri et al.

2014 Journal of Biotechnology 176, 42-49

27 Hamamouch et al.

2012 Journal of Experimental Botany 63, 3683-3695

28 Hu et al.

2013 Experimental Parasitology 135, 15-23

29 Huang et al.
Actin-depolymerizing Factor2-mediated actin dynamics are essential for root-knot nematode infection of Arabidopsis

Silencing of aphid genes by dsRNA feeding from plants

Persistence and transgenerational effect of plant-mediated RNAi in aphids

RNAi-mediated crop improvement for sustainable resistance to Globodera pallida

RNA interference of effector gene Mc16D10L confers resistance against Meloidogyne chitwoodi in Arabidopsis and potato

Plant-mediated RNA interference of effector gene Mc16D10L confers resistance against Meloidogyne chitwoodi in diverse genetic backgrounds of potato and reduces pathogenicity of nematode offspring

Broad Meloidogyne resistance in potato based on RNA interference of effector gene 16D10

Tomato transgenic plants expressing hairpin construct of a nematode protease gene conferred enhanced resistance to root-knot nematodes

Suppression of plant defenses by a Myzus persicae (Green Peach Aphid) salivary effector protein

Host-delivered RNAi: an effective strategy to silence genes in plant parasitic nematodes Plant-Generated artificial small RNAs mediated aphid resistance

Safety assessment of transgenic plums and grapevines expressing viral coat protein genes: New insights into real environmental impact of perennial plants engineered for virus resistance

Citrus tristeza virus-based RNAi in citrus plants induces gene silencing in Diaphorina citri, a phloem-sap sucking insect vector of citrus greening disease (Huanglongbing)

The interaction of the novel 30C02 cyst nematode effector protein with a plant beta1,3-endoglucanase may suppress host defence to promote parasitism

Molecular and biochemical characterization of the beta-1,4-endoglucanase gene Mj-eng3 in the root-knot nematode Meloidogyne javanica

Engineering broad root-knot resistance in transgenic plants by RNAi silencing of a

The present document has been produced and adopted by the bodies identified above as authors. This task has been carried out exclusively by the author(s) in the context of a contract between the European Food Safety Authority and the authors, awarded following a tender procedure. The present document is published complying with the transparency principle to which the Authority is subject. It may not be considered as an output adopted by the Authority. The European Food Safety Authority reserves its rights, view and position as regards the issues addressed and the conclusions reached in the present document, without prejudice to the rights of the authors. 
30 Huang et al.

31 Iberkleid et al.

32 Ibrahim et al.

33 Jaouannet et al.

34 Jin et al.

$35 \quad$ Khajuria et al.

$36 \quad$ Khan et al.

37 Khan et al

38 Klink et al

PLOS ONE 8, e73657

2009 Planta 230, 53-71

39 Konopova and Akam

2014 EvoDevo 5

40 Kumar et al.

2012 PLOS ONE 7, e31347

$41 \quad$ Kyndt et al.

42 Li et al.

2013 Nematology 15, 519-528

2015 Journal of Applied Entomology 139, 432-445

2010 Planta 232, 775-785 conserved and essential root-knot nematode parasitism gene

Molecular cloning and virus-induced gene silencing of MiASB in the southern root-knot nematode, Meloidogyne incognita

Fatty acid- and retinol-binding protein, Mj-FAR-1 induces tomato host susceptibility to root-knot nematodes

Post-transcriptional gene silencing of root-knot nematode in transformed soybean roots

The root-knot nematode calreticulin Mi-CRT is a key effector in plant defense

suppression

Engineered chloroplast dsRNA silences cytochrome p450 monooxygenase, V-ATPase and chitin synthase genes in the insect gut and disrupts Helicoverpa armigera larval development and pupation

Parental RNA interference of genes involved in embryonic development of the western corn rootworm, Diabrotica virgifera virgifera LeConte

Inoculation of Nicotiana tabacum with recombinant potato virus $\mathrm{X}$ induces RNA interference in the solenopsis mealybug, Phenacoccus solenopsis Tinsley (Hemiptera: Pseudococcidae)

Use of recombinant tobacco mosaic virus to achieve RNA interference in plants against the citrus mealybug, Planococcus citri (Hemiptera: Pseudococcidae)

A correlation between host-mediated expression of parasite genes as tandem inverted repeats and abrogation of development of female Heterodera glycines cyst formation during infection of Glycine max

The Hox genes Ultrabithorax and abdominal-A specify three different types of abdominal appendage in the springtail Orchesella cincta (Collembola)

Tobacco Rattle Virus Vector: a rapid and transient means of silencing Manduca sexta genes by plant mediated RNA interference

Transcriptional silencing of RNAi constructs against nematode genes in Arabidopsis Long dsRNA but not siRNA initiates RNAi in western corn rootworm larvae and adults

Host-derived suppression of nematode reproductive and fitness genes decreases fecundity of Heterodera glycines Ichinohe

The present document has been produced and adopted by the bodies identified above as authors. This task has been carried out exclusively by the author(s) in the context of a contract between the European Food Safety Authority and the authors, awarded following a tender procedure. The present document is published complying with the transparency principle to which the Authority is subject. It may not be considered as an output adopted by the Authority. The European Food Safety Authority reserves its rights, view and position as regards the issues addressed and the conclusions reached in the present document, without prejudice to the rights of the authors. 


\section{$44 \quad$ Li et al.}

45 Lin et al.

2010

2013

46 Li et al.

2015

$47 \quad$ Li et al.

48 Lourenco-Tessutti et al.

2015

49 Liu et al.

2015 International Journal of Biological Sciences 11, 67-74

50 Mamta et al

2016 Plant Molecular Biology 90, 281-292

$51 \quad$ Mao \& Zeng

2014 Transgenic Research 23, 145-152

52 Mao et al.

2007 Nature Biotechnology 25, 1307-1313

53 Mao et al.

54 Mao et al.

55 Paim et al.

2013

2013

56 Pandey et al.

57 Pant et al.

$58 \quad$ Pant et al.

2014

2016

Plant Cell Reports 29, 113-123 26, 55-66

( Sciences 11, 1073-1087

PLoS ONE 10, e0129351

Transgenic Research 20, 665-673

Plant Molecular Biology 83, 119-129

Insect Biochemistry and Molecular Biology 43, 1015-1020

Plant Physiology 147, 1212-1224

Plant Signaling \& Behavior 10, e977737

$59 \quad$ Niu et al.
Plant Molecular Biology 85, 107-121

Scientific Reports 6, 19443
Rapid in planta evaluation of root expressed transgenes in chimeric soybean plants A novel effector protein, MJ-NULG1a, targeted to giant cell nuclei plays a role in Meloidogyne javanica parasitism

Cathepsin B cysteine proteinase is essential for the development and pathogenesis of the plant parasitic nematode Radopholus similis

A nematode calreticulin, Rs-CRT, is a key effector in reproduction and pathogenicity of Radopholus similis

Knock-down of heat-shock protein 90 and isocitrate lyase gene expression reduced root-knot nematode reproduction

Silencing the HaAK gene by transgenic plant-mediated RNAi impairs larval growth of Helicoverpa armigera

Targeting chitinase gene of Helicoverpa armigera by host-induced RNA interference confers insect resistance in tobacco and tomato

Plant-mediated RNAi of a gap gene-enhanced tobacco tolerance against the Myzus persicae

Silencing a cotton bollworm P450 monooxygenase gene by plant-mediated RNAi impairs larval tolerance of gossypo

Cotton plants expressing CYP6AE14 double-stranded RNA show enhanced resistance to bollworms

Cysteine protease enhances plant-mediated bollworm RNA interference

Long-term effects and parental RNAi in the blood feeder Rhodnius prolixus (Hemiptera; Reduviidae).

RNA-directed RNA Polymerase3 from Nicotiana attenuata is required for competitive growth in natural environments

The syntaxin 31-induced gene, lesion simulating disease1 (LSD1), functions in Glycine max defense to the root parasite Heterodera glycines

Syntaxin 31 function in glycine max resistance to the plant-parasitic nematode Heterodera glycines

Msp40 effector of root-knot nematode manipulates plant immunity to facilitate parasitism

The present document has been produced and adopted by the bodies identified above as authors. This task has been carried out exclusively by the author(s) in the context of a contract between the European Food Safety Authority and the authors, awarded following a tender procedure. The present document is published complying with the transparency principle to which the Authority is subject. It may not be considered as an output adopted by the Authority. The European Food Safety Authority reserves its rights, view and position as regards the issues addressed and the conclusions reached in the present document, without prejudice to the rights of the authors. 


\section{Papolu et al.}

2013

PLoS ONE 8, e80603

63 Patel et al.

64 Patel et al.

201

Journal of Experimental Botany 61 235-248

2008 Journal of Nematology 40, 299-310

65 Peng et al.

2016 Plos One 11, e0149959

66 Pitino et al.

2011 Plos One 6, e25709

67 Pitino and Hogenhout

2013

68 Pogorelko et al.

69 Rambo-Martin et al.

2016 Molecular plant pathology 17, 832-44

70 Raza et al

71 Porta et al.

72 Shabab et al.

73 Shaheen et al.

74 Shang et al.

75 Sindhu et al.

76 Steeves et al.
2009

In Vitro Cellular \& Developmental Biology-Animal 45, S35-S35

2016 Plos One 11, e0153883

2011 Insect Biochemistry and Molecular Biology 41, 513-519

2014 Febs Journal 281, 2769-2783

2014 Pakistan Entomologist 36, 13-20

2016 Insect molecular biology 25, 422-30

2009 Journal of Experimental Botany 60 315-324

2006 Functional Plant Biology 33, 991-999
Utility of host delivered RNAi of two FMRF amide like peptides, flp-14 and flp-18, for the management of root knot nematode, Meloidogyne incognita

A nematode effector protein similar to annexins in host plants

Similarity and functional analyses of expressed parasitism genes in Heterodera schachtii and Heterodera glycines

Novel pectate lyase genes of Heterodera glycines play key roles in the early stage of parasitism

Silencing of aphid genes by dsRNA feeding from plants

Aphid protein effectors promote aphid colonization in a plant-specific manner

A cyst nematode effector binds to diverse plant proteins, increases nematode susceptibility and affects root morphology

Assessing RNAi gene targets of root-knot nematodes in composite transgenic soybean

RNA Interference based approach to downregulate osmoregulators of whitefly (Bemisia tabaci): potential technology for the control of whitefly

Tobacco plants expressing the Cry1AbMod toxin suppress tolerance to Cry1Ab toxin of Manduca sexta cadherin-silenced larvae

OPDA isomerase GST16 is involved in phytohormone detoxification and insect development

Screening and evaluation of insecticidal RNAi partial gene constructs in non-target insect species

Identification, characterization and functional analysis of a chitin synthase gene in the brown citrus aphid, Toxoptera citricida (Hemiptera, Aphididae)

Effective and specific in planta RNAi in cyst nematodes: expression interference of four parasitism genes reduces parasitic success

Transgenic soybeans expressing siRNAs specific to a major sperm protein gene

The present document has been produced and adopted by the bodies identified above as authors. This task has been carried out exclusively by the author(s) in the context of a contract between the European Food Safety Authority and the authors, awarded following a tender procedure. The present document is published complying with the transparency principle to which the Authority is subject. It may not be considered as an output adopted by the Authority. The European Food Safety Authority reserves its rights, view and position as regards the issues addressed and the conclusions reached in the present document, without prejudice to the rights of the authors. 
77 Tao et al.

2012 Molecular Ecology 21, 4371-4385

78 Thakur et al

2014 Plos One 9, e87235

79 Tian et al.

2015 International Journal of Biological Sciences 11, 1296-1305

80 Tsygankova et al.

81 Tzin et al

$82 \quad$ Urquhart et al.

83 Valentine et al.

84 Vandenborre et al

85 Vastenhouw et al.

86 Vargas et al.

87 Van Eck et al.

88 Vieira et al.

2015 Journal of Insect Physiology 79, 105112

2015 Regulatory Toxicology and

Pharmacology 73, 607-612

2007 Plant Biotechnology Journal 5, 827834

2010 Journal of Experimental Botany 61, 1003-1014

2006 Nature (London) 442, 882

2008 Virology Journal 5

2010 Plant Biotechnology Journal 8, 10231032

2015 Plos One 10, e0144674

2013 Bmc Genomics 14

2014 Journal of Insect Physiology 71, 105113

2015 Pest Management Science 71, 13871396

89 Walawage et
2013 Cytology and Genetics 47, 222-230

suppress Heterodera glycines reproduction

Gossypol-enhanced P450 gene pool contributes to cotton bollworm tolerance to a pyrethroid insecticide

Enhanced whitefly resistance in transgenic tobacco plants expressing double stranded RNA of V-ATPase A gene

Transgenic cotton plants expressing double-stranded RNAs target HMG-CoA reductase (HMGR) gene inhibits the growth, development and survival of cotton bollworms

Increasing the resistance of rape plants to the parasitic nematode Heterodera schachtii using RNAi technology

RNA interference against gut osmoregulatory genes in phloem-feeding insects

A novel method of demonstrating the molecular and functional equivalence between in vitro and plant-produced double-stranded RNA

Delivery of macromolecules to plant parasitic nematodes using a tobacco rattle virus vector

Nicotiana tabacum agglutinin is active against Lepidopteran pest insects

Gene expression: long-term gene silencing by RNAi

Transient expression of homologous hairpin RNA interferes with PVY transmission by aphids

Virus-induced gene silencing of WRKY53 and an inducible phenylalanine ammonia-lyase in wheat reduces aphid resistance

The Pratylenchus penetrans transcriptome as a source for the development of alternative control strategies: mining for putative genes involved in parasitism and evaluation of in planta RNAi

Stacking resistance to crown gall and nematodes in walnut rootstocks

A putative Delta(1)-pyrroline-5-carboxylate synthetase involved in the biosynthesis of proline and arginine in Leptinotarsa decemlineata

Knocking down a putative Delta(1)-pyrroline-5-carboxylate dehydrogenase gene by RNA interference inhibits flight and causes adult lethality in the Colorado potato beetle

The present document has been produced and adopted by the bodies identified above as authors. This task has been carried out exclusively by the author(s) in the context of a contract between the European Food Safety Authority and the authors, awarded following a tender procedure. The present document is published complying with the transparency principle to which the Authority is subject. It may not be considered as an output adopted by the Authority. The European Food Safety Authority reserves its rights, view and position as regards the issues addressed and the conclusions reached in the present document, without prejudice to the rights of the authors. 
92 Wang

93 Woldemariam et al.

$94 \quad$ Wuriyanghan and Falk

95 Xie et al.

96 Xiong et al.

97 Xu et al

98 Xue et al.

99 Yadav et al.

100 Yang et al

101 Youssef et al.

102 Yu et al.

103 Zha et al.

104 Zhang et al.

105 Zhu et al.
2009 Plant Science 176, 279-285

2012 Plant Journal 72, 758-767

2013 Plos One 8, e66050

2016 Frontiers in Plant Science 7

2013 International Journal of Biological Sciences 9, 370-381

2014 Transgenic Research 23, 389-396

2013 Phytopathology 103, 175-181

\section{Molecular and Biochemica} Parasitology 148, 219-222

2013 Plos One 8, e69463

2013 Experimental Parasitology 134, 266274

2014 International Journal of Biological Sciences 10, 1171-1180

2011 Plos One 6, e20504

2015 Science 347, 991-994

2012 PLoS ONE 7, e38572
Leptinotarsa decemlineata (Say)

Monogalactosyldiacylglycerol deficiency affects jasmonic acid biosynthesis and defense responses to insect herbivores in Nicotiana tabacum

Jasmonoyl-I-isoleucine hydrolase 1 (JIH1) regulates jasmonoyl-I-isoleucine levels and attenuates plant defenses against herbivores

RNA interference towards the potato psyllid, Bactericera cockerelli, is induced in plants infected with recombinant Tobacco mosaic virus (TMV)

A novel Meloidogyne incognita effector Misp12 suppresses plant defense response at latter stages of nematode parasitism

Silencing the HaHR3 gene by transgenic plant-mediated RNAi to disrupt Helicoverpa armigera development

Silencing of an aphid carboxylesterase gene by use of plant-mediated RNAi impairs Sitobion avenae tolerance of Phoxim insecticides

The 8D05 parasitism gene of Meloidogyne incognita is required for successful infection of host roots

Host-generated double stranded RNA induces RNAi in plant-parasitic nematodes and protects the host from infection

Molecular characteristics and efficacy of 16D10 siRNAs in inhibiting root-knot nematode infection in transgenic grape hairy roots

Post-transcriptional gene silencing of the gene encoding aldolase from soybean cyst nematode by transformed soybean roots

The insect ecdysone receptor is a good potential tTarget for RNAi-based pest control

Knockdown of midgut genes by dsRNA-transgenic plant-mediated RNA interference in the Hemipteran Insect Nilaparvata lugens

Full crop protection from an insect pest by expression of long double-stranded RNAs in plastids

Improvement of pest resistance in transgenic tobacco plants expressing dsRNA of an insect-associated gene EcR

The present document has been produced and adopted by the bodies identified above as authors. This task has been carried out exclusively by the author(s) in the context of a contract between the European Food Safety Authority and the authors, awarded following a tender procedure. The present document is published complying with the transparency principle to which the Authority is subject. It may not be considered as an output adopted by the Authority. The European Food Safety Authority reserves its rights, view and position as regards the issues addressed and the conclusions reached in the present document, without prejudice to the rights of the authors. 


\section{Appendix D - \\ List of publications considered relevant for Task 3 "Environmental exposure and fate of dsRNA} siRNA and miRNA" - Environmental fate

\begin{tabular}{|c|c|c|c|c|c|}
\hline No. & Authors & Year & Journal, volume, pages & Title & Relevance \\
\hline 1 & Ashe et al. & 2012 & Cell (Cambridge) 150, 88-99 & $\begin{array}{l}\text { piRNAs can trigger a multigenerational epigenetic memory in } \\
\text { the germline of Caenorhabditis elegans }\end{array}$ & $\begin{array}{l}\text { Multigenerational } \\
\text { epigenetic effects }\end{array}$ \\
\hline 2 & Ashe et al. & 2015 & $\begin{array}{l}\text { Journal of Virology } 89,12035- \\
12046\end{array}$ & $\begin{array}{l}\text { Antiviral RNA interference against Orsay virus ss neither } \\
\text { systemic nor transgenerational in Caenorhabditis elegans }\end{array}$ & $\begin{array}{l}\text { Lack of transgenerational } \\
\text { effects }\end{array}$ \\
\hline 3 & Bosch et al. & 2016 & Genetics $203,109-118$ & $\begin{array}{l}\text { Persistence of RNAi-Mediated knockdown in Drosophila } \\
\text { complicates mosaic analysis yet enables highly sensitive } \\
\text { lineage tracing }\end{array}$ & $\begin{array}{l}\text { Transgenerational } \\
\text { effects/Epigenetics }\end{array}$ \\
\hline 4 & Buckley et al. & 2012 & Nature (London) 489, 447-451 & $\begin{array}{l}\text { A nuclear Argonaute promotes multigenerational epigenetic } \\
\text { inheritance and germline immortality }\end{array}$ & $\begin{array}{l}\text { Transgenerational } \\
\text { effects/Epigenetics }\end{array}$ \\
\hline 5 & Burton et al. & 2011 & $\begin{array}{l}\text { Proceedings of the National } \\
\text { Academy of Sciences of the } \\
\text { United States of America 108, } \\
\text { 19683-19688 }\end{array}$ & $\begin{array}{l}\text { Nuclear RNAi maintains heritable gene silencing in } \\
\text { Caenorhabditis elegans }\end{array}$ & $\begin{array}{l}\text { Transgenerational } \\
\text { effects/Epigenetics }\end{array}$ \\
\hline 6 & Dubelman et al. & 2014 & Plos One 9, e93155 & $\begin{array}{l}\text { Environmental fate of double-stranded RNA in agricultural } \\
\text { soils }\end{array}$ & Environmental fate \\
\hline 7 & Gamboa Cedeno et al. & 2015 & $\begin{array}{l}\text { Journal of Apicultural Research } \\
54,99-100\end{array}$ & $\begin{array}{l}\text { Double-stranded RNA synthesized in bacteria can be } \\
\text { transferred to bee and Varroa tissues }\end{array}$ & Trophic chains \\
\hline 8 & Garbian et al. & 2012 & Plos Pathogens 8, e1003035 & $\begin{array}{l}\text { Bidirectional transfer of RNAi between honey bee and Varroa } \\
\text { destructor: Varroa gene silencing reduces Varroa population }\end{array}$ & Trophic chains \\
\hline 9 & Grishok et al. & 2000 & Science $287,2494-2497$ & $\begin{array}{l}\text { Genetic requirements for inheritance of RNAi in } \\
\text { Caenorhabditis elegans }\end{array}$ & $\begin{array}{l}\text { Transmission of effects to } \\
\text { offsprings }\end{array}$ \\
\hline 10 & Houri-Ze'evi et al. & 2016 & Cell 165, 88-99 & $\begin{array}{l}\text { A tunable mechanism determines the duration of the } \\
\text { transgenerational small RNA inheritance in Caenorhabditis } \\
\text { elegans }\end{array}$ & Transgenerational effects \\
\hline 11 & Itakura et al. & 2009 & Sociobiology $54,77-87$ & $\begin{array}{l}\text { RNA Interference in symbiotic protists of the termite } \\
\text { Coptotermes formosanus (Isoptera: Rhinotermitidae) } \\
\text { through ingestion of siRNA by the host termite }\end{array}$ & Tritrophic \\
\hline 12 & Jia et al. & 2015 & Scientific Reports 5, 12290 & $\begin{array}{l}\text { Nonfunctional ingestion of plant miRNAs in silkworm revealed } \\
\text { by digital droplet PCR and transcriptome analysis }\end{array}$ & $\begin{array}{l}\text { Cross-kingdom transfer of } \\
\text { endogenous RNA }\end{array}$ \\
\hline www. & sa.europa.eu/publications & & & EFSA Suppoi & ting publication 2018:EN-1424 \\
\hline
\end{tabular}


13 Khajuria et al.

$14 \quad$ Li et al.

15 San Miguel and Scott
2015 Insect Biochemistry and

Molecular Biology 63, 54-62

2015 Plant Cell and Environment 38, 2277-2285

2016 Pest Management Science 72 801-809
Parental RNA interference of genes involved in embryonic development of the western corn rootworm, Diabrotica

virgifera virgifera LeConte

New insights into an RNAi approach for plant defence against piercing-sucking and stem-borer insect pests

The next generation of insecticides: dsRNA is stable as a foliar-applied insecticide
Transgeneration

dsRNA stability under different conditions

Persistence after plant spray or absorption

The present document has been produced and adopted by the bodies identified above as authors. This task has been carried out exclusively by the author(s) in the context of a contract between the European Food Safety Authority and the authors, awarded following a tender procedure. The present document is published complying with the transparency principle to which the Authority is subject. It may not be considered as an output adopted by the Authority. The European Food Safety Authority reserves its rights, view and position as regards the issues addressed and the conclusions reached in the present document, without prejudice to the rights of the authors. 
Appendix E - List of publications deemed relevant for Task 5 "Off target, non-target and unintended effects of RNAi-based GM plants"

\begin{tabular}{|c|c|c|c|c|c|}
\hline No. & Authors & Year & Journal, volume, pages & Title & Relevance \\
\hline 1 & Ahmad et al. & 2016 & Transgenic research $25,1-17$ & $\begin{array}{l}\text { Transportable data from non-target arthropod field studies } \\
\text { for the environmental risk assessment of genetically modified } \\
\text { maize expressing an insecticidal double-stranded RNA }\end{array}$ & Non-target \\
\hline 2 & $\begin{array}{l}\text { Antonino de Souza Junior } \\
\text { et al. }\end{array}$ & 2013 & PLoS ONE 8, e85364 & $\begin{array}{l}\text { Knocking-Down Meloidogyne incognita Proteases by Plant- } \\
\text { Delivered dsRNA Has Negative Pleiotropic Effect on } \\
\text { Nematode Vigor }\end{array}$ & Off-target \\
\hline 3 & Asokan et al. & 2012 & Current Science 102, 1692-1699 & $\begin{array}{l}\text { Common siRNAs for various target genes of the fruit borer, } \\
\text { Helicoverpa armigera Hubner (Lepidoptera: Noctuidae) }\end{array}$ & $\begin{array}{l}\text { Remedies/minimizing } \\
\text { off-target }\end{array}$ \\
\hline 4 & Bachman et al. & 2013 & $\begin{array}{l}\text { Transgenic Research 22, 1207- } \\
1222\end{array}$ & $\begin{array}{l}\text { Characterization of the spectrum of insecticidal activity of a } \\
\text { double-stranded RNA with targeted activity against Western } \\
\text { Corn Rootworm (Diabrotica virgifera virgifera LeConte) }\end{array}$ & Specificity \\
\hline 5 & Chen et al. & 2015 & Ecotoxicology 24, 595-603 & $\begin{array}{l}\text { The effects of RNA interference targeting Bactrocera dorsalis } \\
\text { ds-Bdrpl19 on the gene expression of rpl19 in non-target } \\
\text { insects }\end{array}$ & Non-target \\
\hline 6 & Chen et al. & 2011 & $\begin{array}{l}\text { Developmental and Comparative } \\
\text { Immunology } 35,661-671\end{array}$ & $\begin{array}{l}\text { Identification and functional characterization of Dicer2 and } \\
\text { five single VWC domain proteins of Litopenaeus vannamei }\end{array}$ & $\begin{array}{l}\text { Immune } \\
\text { stimulation }\end{array}$ \\
\hline 7 & Danchin et al. & 2013 & PLoS Pathogens 9, e1003745 & $\begin{array}{l}\text { Identification of Novel Target Genes for Safer and More } \\
\text { Specific Control of Root-Knot Nematodes from a Pan- } \\
\text { Genome Mining }\end{array}$ & $\begin{array}{l}\text { Non-target } \\
\text { Bioinformatics }\end{array}$ \\
\hline 8 & DasGupta et al. & 2007 & Genome Biology 8, R203 & $\begin{array}{l}\text { A case study of the reproducibility of transcriptional reporter } \\
\text { cell-based RNAi screens in Drosophila }\end{array}$ & $\begin{array}{l}\text { Non-target } \\
\text { Bioinformatics }\end{array}$ \\
\hline 9 & Deddouche et al. & 2008 & Nature Immunology 9, 1425-1432 & $\begin{array}{l}\text { The DExD/H-box helicase Dicer-2 mediates the induction of } \\
\text { antiviral activity in Drosophila }\end{array}$ & $\begin{array}{l}\text { Immune } \\
\text { stimulation }\end{array}$ \\
\hline 10 & Dong et al. & 2009 & $\begin{array}{l}\text { Fish \& Shellfush Immunology } 26, \\
438-442\end{array}$ & $\begin{array}{l}\text { The immune responses in Chinese mitten crab Eriocheir } \\
\text { sinensis challenged with double-stranded RNA }\end{array}$ & $\begin{array}{l}\text { Immune } \\
\text { stimulation }\end{array}$ \\
\hline 11 & Fisher et al. & 2012 & BMC Genomics 13, nr. 506 & $\begin{array}{l}\text { Advances in genome-wide RNAi cellular screens: a case } \\
\text { study using the Drosophila JAK/STAT pathway }\end{array}$ & $\begin{array}{l}\text { Off-target } \\
\text { Bionformatics }\end{array}$ \\
\hline 12 & Flenniken et al. & 2013 & PLoS One 8, e77263 & $\begin{array}{l}\text { Non-Specific dsRNA-Mediated Antiviral Response in the } \\
\text { Honey Bee }\end{array}$ & $\begin{array}{l}\text { Immune } \\
\text { stimulation }\end{array}$ \\
\hline www & sa.europa.eu/publications & \multicolumn{4}{|r|}{ 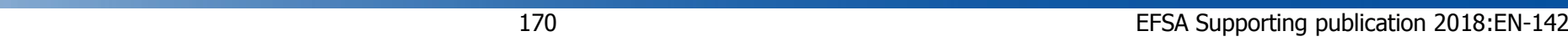 } \\
\hline
\end{tabular}


Journal of Apicultural Research

$54,99-100$

14 Garbian et al.

Plos Pathogens 8, e1003035

15 Garbutt et al.

nsect Biochemistry and Molecular Biology 42, 621-628

16 Hirai et al.

17 Jarosch et al. 405

18 Kingsolver et al.

Apidologie 43, 128-138

19 Kitzman et al.

Kola et al.

Developmental and Comparative Immunology 34, 1209-1218

PLoS ONE 10, e0118622

Journal of Insect Physiology 59, 646-654

24 Luan et al.

2012 Development Genes and Evolution 222, 229-235
2007 Trends in Pharmacological Sciences 28, 149-151
Double-stranded RNA synthesized in bacteria can be transferred to bee and Varroa tissues

Bidirectional Transfer of RNAi between Honey Bee and Varroa destructor: Varroa Gene Silencing Reduces Varroa Population

Induction of RNA interference genes by double-stranded

RNA; implications for susceptibility to RNA interference

Baculovirus and dsRNA induce Hemolin, but no antibacterial activity, in Antheraea pernyi

RNA interference in honeybees: off-target effects caused by dsRNA

Insect Antiviral Innate Immunity: Pathways, Effectors, and

Connections

RNAi phenotypes are influenced by the genetic background of the injected strain

Silencing of CYP6 and APN Genes Affects the Growth and

Development of Rice Yellow Stem Borer, Scirpophaga incertulas

Non-specific activation of antiviral immunity and induction of

RNA interference may engage the same pathway in the

Pacific white leg shrimp Litopenaeus vannamei

Independent Action between DvSnf7 RNA and Cry3Bb1

Protein in Southern Corn Rootworm, Diabrotica

undecimpunctata howardi and Colorado Potato Beetle,

Leptinotarsa decemlineata

Transcriptional response of BmTo119-1 and RNAi machinery

genes to exogenous dsRNA in the midgut of Bombyx mori

The Unique GGA Clathrin Adaptor of Drosophila

melanogaster Is Not Essential

Super-induction of Dicer-2 expression by alien double-

stranded RNAs: an evolutionary ancient response to viral

infection?

Off-target effects associated with long dsRNAs in Drosophila

RNAi screens
Trophic chain

Trophic chain

Immune stimulation Immune stimulation Off-target

Immune stimulation Resistance mechanism Non-target

Immune stimulation

Non-target

Immune stimulation Off-target

Immune stimulation

Off-target

The present document has been produced and adopted by the bodies identified above as authors. This task has been carried out exclusively by the author(s) in the context of a contract between the European Food Safety Authority and the authors, awarded following a tender procedure. The present document is published complying with the transparency principle to which the Authority is subject. It may not be considered as an output adopted by the Authority. The European Food Safety Authority reserves its rights, view and position as regards the issues addressed and the conclusions reached in the present document, without prejudice to the rights of the authors. 
27 Nunes et al.

2013 Insects 4, 90-103

28

Piot et al.

2015

Qiu et al.

30 Robalino et al.

Robalino et al.

32

34

Su et al.

37 1847 13571

2007

2011

Runo et al.

Seinen et al

Seinen et al.

Velez et al.

2009

2016

2009
Nucleic Acids Research 33, 1834

2007 Physiological Genomics 29, 44-56

2005 Journal of Virology 79, 13561-

$289,1-5$

PLoS ONE 11, e0157520

Insect Biochemistry and Molecular Biology 39, 824-832

2015 Entomologia Experimentalis Et

Applicata 155, 218-228

2014 Genetics 197, 121-132
Non-Target Effects of Green Fluorescent Protein (GFP)Derived Double-Stranded RNA (dsRNA-GFP) Used in Honey Bee RNA Interference (RNAi) Assays

The Effect of Oral Administration of dsRNA on Viral Replication and Mortality in Bombus terrestris

A computational study of off-target effects of RNA interference

Insights into the immune transcriptome of the shrimp Litopenaeus vannamei: tissue-specific expression profiles and transcriptomic responses to immune challenge

Double-stranded RNA induces sequence-specific antiviral silencing in addition to nonspecific immunity in a marine shrimp: Convergence of RNA interference and innate immunity in the invertebrate antiviral response?

Novel insights into RNAi off-target effects using C-elegans paralogs

RNA interference as a resistance mechanism against crop parasites in Africa: a 'Trojan horse' approach

RNAi-induced off-target effects in Drosophila melanogaster frequencies and solutions

RNAi Experiments in D. melanogaster: Solutions to the Overlooked Problem of Off-Targets Shared by Independent dsRNAs

Grass carp reovirus activates RNAi pathway in rare minnow, Gobiocypris rarus

Knockdown of RNA Interference Pathway Genes in Western Corn Rootworms (Diabrotica virgifera virgifera Le Conte)

Demonstrates a Possible Mechanism of Resistance to Lethal dsRNA

Ingested double-stranded RNAs can act as species-specific insecticides

Lepidopteran insect species-specific, broad-spectrum, and systemic RNA interference by spraying dsRNA on larvae Nuclear RNAi Contributes to the Silencing of Off-Target
Non-target/off-target

Immune stimulation Off-target

Immune stimulation

Immune stimulation

Off-target Bionformatics

Specificity

Remedies/minimizing off-target

Remedies/minimizing off-target

Immune stimulation Non-target

Specificity

Specificity

Off-target

The present document has been produced and adopted by the bodies identified above as authors. This task has been carried out exclusively by the author(s) in the context of a contract between the European Food Safety Authority and the authors, awarded following a tender procedure. The present document is published complying with the transparency principle to which the Authority is subject. It may not be considered as an output adopted by the Authority. The European Food Safety Authority reserves its rights, view and position as regards the issues addressed and the conclusions reached in the present document, without prejudice to the rights of the authors. 


\section{Zhu et al.}

The present document has been produced and adopted by the bodies identified above as authors. This task has been carried out exclusively by the author(s) in the context of a contract between the European Food Safety Authority and the authors, awarded following a tender procedure. The present document is published complying with the transparency principle to which the Authority is subject. It may not be considered as an output adopted by the Authority. The European Food Safety Authority reserves its rights, view and position as regards the issues addressed and the conclusions reached in the present document, without prejudice to the rights of the authors. 